\title{
Platelets and coagulation
}

Citation for published version (APA):

Swieringa, F. (2016). Platelets and coagulation: partners in haemostasis. [Doctoral Thesis, Maastricht University]. https://doi.org/10.26481/dis.20160331fs

Document status and date:

Published: 01/01/2016

DOI:

$10.26481 /$ dis.20160331fs

Document Version:

Publisher's PDF, also known as Version of record

\section{Please check the document version of this publication:}

- A submitted manuscript is the version of the article upon submission and before peer-review. There can be important differences between the submitted version and the official published version of record.

People interested in the research are advised to contact the author for the final version of the publication, or visit the DOI to the publisher's website.

- The final author version and the galley proof are versions of the publication after peer review.

- The final published version features the final layout of the paper including the volume, issue and page numbers.

Link to publication

\footnotetext{
General rights rights.

- You may freely distribute the URL identifying the publication in the public portal. please follow below link for the End User Agreement:

www.umlib.nl/taverne-license

Take down policy

If you believe that this document breaches copyright please contact us at:

repository@maastrichtuniversity.nl

providing details and we will investigate your claim.
}

Copyright and moral rights for the publications made accessible in the public portal are retained by the authors and/or other copyright owners and it is a condition of accessing publications that users recognise and abide by the legal requirements associated with these

- Users may download and print one copy of any publication from the public portal for the purpose of private study or research.

- You may not further distribute the material or use it for any profit-making activity or commercial gain

If the publication is distributed under the terms of Article $25 \mathrm{fa}$ of the Dutch Copyright Act, indicated by the "Taverne" license above, 


\section{PLATELETS AND COAGULATION PARTNERS IN HAEMOSTASIS}




\section{PLATELETS AND COAGULATION PARTNERS IN HAEMOSTASIS}

Thesis Universiteit Maastricht

ISBN 978-94-6295-331-4

Uitgeverij BOXPress || Proefschriftmaken.nl

(c) Frauke Swieringa, Maastricht 2016

Cover by Willy Swieringa-Lamers 


\title{
PLATELETS AND COAGULATION
} PARTNERS IN HAEMOSTASIS

\author{
PROEFSCHRIFT
}

ter verkrijging van de graad van doctor aan de Universiteit Maastricht, op gezag van de Rector Magnificus, Prof. Dr. L.L.G. Soete, volgens het besluit van het College van Decanen, in het openbaar te verdedigen op

donderdag 31 maart 2016 om 14:00

door

Frauke Swieringa

Geboren op 23 oktober 1984 te Heerlen 


\section{Promotor}

Prof. Dr. J.W.M. Heemskerk

\section{Copromotor}

Dr. P.E.J. van der Meijden

\section{Beoordelingscommissie}

Prof. Dr. H.C. Schouten (voorzitter)

Prof. Dr. R.A.S. Ariëns (University of Leeds, United Kingdom)

Prof. Dr. R.W. Farndale (University of Cambridge, United Kingdom)

Dr. Ir. Y.M.C. Henskens

Dr. R.R. Koenen

This project was supported by the Dutch Landsteiner Foundation for Blood Transfusion Research.

Financial support by Stichting Hart Onderzoek Nederland, an initiative of the Heart Research Institute (www.hartonderzoek.nu), is gratefully acknowledged.

Financial support for publication of this thesis by Stichting Fonds voor het Hart is gratefully acknowledged. 
Haemoglobin is the key to a healthy heart beat

- Placebo 



\section{CONTENTS}

$\begin{array}{llr}\text { Chapter } 1 \text { General introduction } & 9\end{array}$

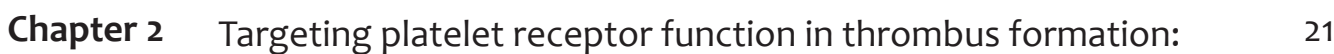
The risk of bleeding

Chapter 3 Multiple changes in novel protein kinase A phosphorylation targets identified in platelets from patients with impaired Gsa signalling

Chapter 4 Desmopressin treatment improves platelet function under flow in patients with postoperative bleeding

Chapter 5 Key role of integrin $\alpha_{\| 1 \mathrm{~b}} \beta_{3}$ signalling to Syk kinase in tissue 103 factor-induced thrombin generation

Chapter 6 Platelet control of fibrin distribution and microelasticity in thrombus formation under flow

Chapter 7 Rate-limiting roles of tenase complex of factors VIII and IX in platelet procoagulant activity and formation of platelet-fibrin thrombi

Chapter 8 Plasminogen associates with phosphatidylserine-exposing 173 platelets and contributes to thrombus lysis under flow

$\begin{array}{lll}\text { Chapter } 9 \text { General discussion } & 197\end{array}$

$\begin{array}{lll}\text { Chapter } 10 & \text { Summary } & 217\end{array}$

Samenvatting 223

Valorisation 229

Curriculum vitae $\quad 235$

$\begin{array}{ll}\text { Publications } & 239\end{array}$

Dankwoord 247 



\section{CHAPTER 1}

General introduction 
Haemostasis refers to the cessation of blood loss after tissue and vascular injury. The process of blood clotting in haemostasis is tightly controlled by the activity of platelets and the coagulation system. The underlying mechanisms of clot formation are still only partly understood, but are based on the adhesion of platelets to extracellular matrix components such as collagen and the initiation of coagulation by extravascular ${ }^{1,2}$ or blood-borne ${ }^{3}$ tissue factor. Platelets subsequently aggregate into a haemostatic plug, and the activated coagulation cascade produces thrombin and fibrin, forming the platelet-fibrin thrombus. ${ }^{4}$ In the majority of patients with bleeding complications, one or both of these processes are flawed with as a result hampered thrombus formation. A similar cooperativity of platelet activation and coagulation occurs in thrombotic diseases, caused by the occlusion of an intact artery or vein in the absence of blood loss. Thrombus formation in this case, however, is influenced by the location in the circulation, as platelet activation predominates in arterial thrombi where the wall shear rate of the blood is high, whereas coagulation with fibrin formation predominates in venous thrombi where the blood shear rate is low. ${ }^{5,6}$ To provide a general background on the mechanisms of platelet activation and coagulation activity in thrombus formation, this chapter will focus on: (i) the key platelet receptors in thrombus formation; (ii) the use of modern proteomics techniques to identify the proteins involved; (iii) key steps in thrombin generation, fibrin formation and fibrinolysis; and (iv) the interactions between platelets and coagulation in thrombus formation. These topics will be introduced in a concise way, only as far as relevant for the various chapters of this thesis.

\section{Key platelet receptors in thrombus formation}

There is no doubt that platelets can interact with multiple ligands via dozens of receptors and then become activated in terms of shape change, aggregation and secretion. ${ }^{7,8}$ Several of these receptor interactions appear to be of major importance for the formation of a multi-platelet thrombus on collagen surfaces both in vitro and in vivo. ${ }^{4,9,10}$ Principal platelet-activating pathways are triggered via adhesive receptors, often acting via protein tyrosine kinases, and via $G$ protein-coupled receptors, regulating second messengers such as $\mathrm{Ca}^{2+}$, diacylglycerol and cyclic AMP.

Von Willebrand factor (VWF) initiates collagen-induced thrombus formation under high shear conditions by binding to collagen. Platelet adhesion to collagenbound VWF is mediated by the glycoprotein Ib-V-IX complex (>20,000 copies per platelet), followed by $14-3-3 \zeta$ signalling, eliciting limited platelet responses via phospholipase $C_{\gamma}$ and phosphatidylinositol 3-kinase. As VWF is responsible for the initial platelet-collagen interaction it can be hypothesised that a VWF stimulating agent, e.g. desmopressin, could aid haemostasis under conditions of a diminished thrombus forming potential. This is highlighted by impaired haemostasis, i.e. increased bleeding risk, in patients with Bernard-Soulier syndrome and von Willebrand disease attributed to abnormalities in the GPIb-V-IX complex or VWF, respectively. ${ }^{11}{ }^{12}$ 
The tyrosine kinase-activating receptors glycoprotein $\mathrm{VI}$ for collagen, FcyRIIA for immune complexes and CLEC-2 for podoplanin contain or associate with coreceptors containing (hem)ITAM groups that establish an interaction with Src family kinases. ${ }^{13-15}$. Via activation of phospholipase $C_{\gamma}$ and phosphatidylinositol 3-kinase this strongly promotes platelet adhesion, granule secretion, integrin activation and phosphatidylserine exposure, crucial for full thrombus formation. Platelets bind to collagen not only via glycoprotein $\mathrm{VI}$, but also via integrin $\alpha_{2} \beta_{1}$, which ensures stable adhesion to collagen with limited signalling. ${ }^{16,17}$

An activating conformational change of integrin $\alpha_{11 b} \beta_{3}$ is one of the results of platelet activation. The integrin $\alpha_{11 b} \beta_{3}$ acts as a receptor for fibrinogen, VWF and fibronectin and is essential for platelet aggregation and the formation of a thrombus. ${ }^{18}$ This becomes more apparent from patients with Glanzmann's thrombasthenia, with a deficiency or dysfunction of the integrin, who suffer from bleeding complications. Also mice lacking the functional integrin or its ligand fibrinogen suffer bleeding complications as thrombus formation is absent. ${ }^{19-21}$ Binding of fibrinogen to its receptor drives multiple processes, including the spreading of platelets on fibrinogen and the retraction of fibrin clots. While it is well appreciated that blocking of $\alpha_{11 b} \beta_{3}$ supresses tissue factor-induced thrombin generation, the mechanism by which this interferes with platelet-dependent coagulation has not been resolved.

Soluble platelet agonists, such as thrombin, ADP and thromboxane $A_{2}$, stimulate platelets via $G$ protein-coupled receptors. Thrombin protease-activated receptors (PAR)1/PAR4, and the thromboxane $A_{2}$ TP receptor all link to $\mathrm{G}_{12} / 13$ and $\mathrm{Gq}$ proteins, while ADP receptors $P_{2} Y_{1}$ and $P_{2} Y_{12}$ link to $G q$ and $G i$ proteins. The heterotrimeric $G$-proteins, composed of an $\alpha, \beta$ and $\gamma$ subunit, dissociate upon agonist binding to the receptor, leaving the surfaces of both the $a$ and the $\beta \gamma$ subunits exposed for interactions with effector proteins. The function and identity of the a subunit is what separates $G$ proteins in $\mathrm{G} 12 / 13, \mathrm{Gi}, \mathrm{Gq}$ and $\mathrm{Gs}{ }^{7}$ In short, $\mathrm{G} 12 / 13$ signalling is mostly associated with platelet shape change, via activation of Rho-associated protein kinase. ${ }^{22}$ Signalling via $\mathrm{Gi}$ inhibits adenylyl cyclase and stimulates the phosphatidylinositol 3-kinase pathway, which links to integrin activation. Gq signals via phospholipase $C \beta$ increasing cytosolic $\mathrm{Ca}^{2+}$ (via inositol-1,4,5-trisphosphate) and activates protein kinase C (via diacylglycerol). Both $\mathrm{Ca}^{2+}$ and protein kinase $\mathrm{C}$ are implicated in platelet secretion and integrin activation, and, hence platelet aggregation. ${ }^{4,8}$ The $\mathrm{G}$ proteins $\mathrm{Gi}$ and $\mathrm{Gq}$ thereby contribute to processes critical for thrombus formation by mediating shape change, granule secretion as well as integrin activation and platelet aggregation.

Signalling to procoagulant activity depends on dual stimulation of the tyrosine kinase-activating receptors and $G$ protein-coupled receptors, as occurs with stimulation of collagen/thrombin, evoking prolonged and high rises in cytosolic $\mathrm{Ca}^{2+}$ concentration. The platelet procoagulant activity is then the result of exposure of the negatively charged phosphatidylserine at the membrane surface via a transmembrane protein encoded by TMEM16F. This promotes the local assembly of vitamin K-dependent coagulation factors and thereby the generation of thrombin. 
Also platelet-inhibitory pathways exist in order to prevent uncontrolled platelet activation by limiting platelet function and thrombus formation in the circulation where the endothelium is intact. Prostacyclin (prostaglandin $I_{2}$ ) is produced by endothelial cells from prostaglandin $\mathrm{H}_{2}$ by the enzyme prostacyclin synthase and interacts with the platelet IP receptor coupled to Gs. ${ }^{23}$ Prostacyclin limits platelet activation through the formation of cyclic adenosine 3',5'-monophosphate (cAMP) and stimulation of protein kinase A (PKA). It has been demonstrated in vivo that elevations in platelet CAMP coincide with reduced $\mathrm{Ca}^{2+}$ mobilisation, dense granule secretion and activation of integrin $\alpha_{11 \mathrm{~b}} \beta_{3}$. On the other hand, reduction in CAMP/PKA signalling, i.e. in $\mathrm{PGI}_{2}$ receptor (IP) deficient mice, is associated with enhanced platelet activation.

The Gsa protein is encoded by a complex imprinted locus, GNAS, along with several different gene products, i.e. extra-large Gsa, neuroendocrine secretory protein NESP55, the noncoding $\mathrm{A} / \mathrm{B}$ and antisense transcripts. The promotor of NESP55 is exclusively maternally expressed while the promotor of transcripts shows only paternal expression. The Gsa promoter is not methylated and thus the transcript is biallelically expressed in most tissues. Interestingly, in some tissues such as proximal renal tubes, thyroid and gonads, the paternal Gsa expression is silenced. ${ }^{24-26}$ The protein Gsa, encoded by GNAS exons 1-13, mediates not only platelet function but also the actions of many hormones and other endogenous molecules via CAMP generation. Genetic alterations affecting GNAS are responsible for a heterogeneous group of disorders known as pseudohypoparathyroidism (PHP). These disorders are characterised by impaired signalling of various hormones, mainly parathyroid hormone, that stimulate Gs-dependent CAMP pathways, with the phenotype depending on the parent transmitting the mutation. Albright hereditary osteodystrophy (AHO) is a clinical entity associated with brachydactyly, short stature, rounded face, central obesity and a variable degree of mental retardation. When accompanied by multiple hormone resistance (maternal transmission) the disease is indicated as PHP type la, while in pseudopseudohypoparathyroidism (PPHP) there is no hormone resistance (paternal transmission). ${ }^{27,28}$

\section{Use of proteomics to identify (platelet) protein kinases in thrombus formation}

Haemostasis greatly depends on the platelets' potential to swiftly adapt to an ever changing environment. While their protein abundance is obviously dependent on transcription, translation and degradation, their activity and hence their function, is regulated by post-translational modifications (PTMs). Most prominently, these include phosphorylation, glycosylation and proteolytic cleavage and enable platelets to respond to stimuli within (milli)seconds. Current knowledge about the dynamic presence of protein PTMs has been considerably extended by mass spectrometrybased proteomics. While initially quantitative proteomics was predominantly used to study the role of aberrant PTM patterns in human disease (previously confined 
to individual proteins or defined pathways), both the technological and methodical progress have generated now PTM-specific proteomic studies. Knowledge about PTM involvement in disease mechanisms is mainly derived from in vitro or animal models, as most strategies have focussed on cell lines. With the new strategies requiring little sample amount they show a great potential to discover, validate and accurately quantify biomarkers in body fluids and primary tissues also in a clinical setting. ${ }^{29,} 30$ For this application it is critical to ensure a sensitive but robust test. Thus, one future challenge will be to identify and validate novel models directly in clinical samples, allowing the identification and verification of biomarker candidates and drug targets.

\section{Thrombin generation, fibrin formation and fibrinolysis}

The coagulation process is executed by a cascade of enzymatic reactions, wherein proenzymes of serine proteases are cleaved into the active proteases, culminating in the formation of thrombin as a central player. ${ }^{31}$ Two routes trigger the generation of thrombin, i.e. the intrinsic and the extrinsic pathway. The intrinsic (or contact activation) pathway is triggered in vitro by blood contact with negatively charged surfaces such as glass or kaolin, which auto-activate factor XII (kaolin was used for coagulation assays in the clinic in the activated partial thromboplastin time or aPTT). ${ }^{32}$ In vivo, the intrinsic pathway of factor XII activation can occur by subendothelial collagens, ${ }^{33}$ polyphosphates, ${ }^{34}$ glycosaminoglycans and proteoglycans ${ }^{35}$ or RNA. ${ }^{36,37}$ The initial activation involves a reaction with high molecular weight kininogen and plasma kallikrein to cleave factor XII. Once activated, factor XIla can activate factor $\mathrm{XI}$, a reaction occurring during thrombus formation, ${ }^{33}$ which acts as a protease to activate factor IX. It is generally considered that activated, phosphatidylserineexposing platelets serve as a surface for the activation of factor $X$ by factor IXa and cofactor VIIIa. ${ }^{38}$ Factor VIII (absent in patients with haemophilia A) is an interesting haemostatic protein, as it remains in the circulation in complex with VWF. ${ }^{39}$ In which way the formation and activity of the so-called tenase complex (FVIIIa/FIXa) is regulated under conditions of thrombus formation is still incompletely understood.

The extrinsic coagulation pathway towards initial factor $X$ activation proceeds by a shorter route, by complex formation of tissue factor with factor VII(a). ${ }^{40}$ Once initial traces of factor $X a$ and its substrate thrombin are formed, other coagulation factors such as factor V, factor VIII and factor XI can be cleaved and activated as well. Both factor Xa and co-factor $V a$ assemble on the surface of phosphatidylserine-exposing platelets to stimulate the cleavage of prothrombin into thrombin. As reviewed elsewhere, it has been calculated that the prothrombinase complex formed on a phosphatidylserine surface enhances the thrombin formation more than thousandfold. ${ }^{1,}{ }^{41}$ The strongly enhanced local generation of thrombin activates more platelets and provides a strong feed forward mechanism of the coagulation cascade. ${ }^{42}$ Many other positive and negative feedback loops exist in this cascade, making the whole process of thrombin and fibrin generation very dynamic, but describing these is 
beyond the scope of this thesis.

The formation of fibrin fibres is a complex process, described in detail elsewhere. ${ }^{43}$ It starts with the cleavage of large fibrinogen molecules - either bound to platelets or free in plasma - by thrombin into fibrin monomers. These monomers arrange and align to form microscopic fibres, of which a last step is the cross-linking of the aligned fibrils by the transglutaminase, factor XIII. Fibrin crosslinking is considered to be essential for stable fibrin clot formation. ${ }^{44} \mathrm{~A}$ high degree of crosslinking and high viscoelasticity of the fibrin fibre network is critical for achieving sufficient haemostatic activity and to prevent bleeding. ${ }^{45}$ This property is a function of the fibrinogen and prothrombin levels in plasma, the extent of thrombin generation, and the local blood flow. ${ }^{46}$ The conditions required for the formation of a thrombus with high viscoelasticity however are not well known.

Fibrinolysis is the process of fibrin network degradation, which is also mediated by serine proteases with positive and negative feedback loops. The central pathway is the cleavage of (circulating) plasminogen into plasmin, which is the major fibrinolytic protease. Interestingly, plasmin not only cleaves fibrin, but also fibrinogen, factors $V$ and VIII, integrin $\alpha_{11 b} \beta_{3}$ and other proteins. ${ }^{47}$ Plasminogen cleavage depends mostly on tissue-type plasminogen activator (tPA) and to a lesser extent by urokinase-type plasminogen activator (UPA) and is promoted by the presence of fibrin, as both tPA and plasminogen bind to the free lysine residues of fibrin fibres, of which the number increases during fibrin cleavage. ${ }^{48}$ Accordingly, during the lysis of fibrin more plasminogen can bind and thus become converted into plasmin. Physiologically relevant inhibitors of plasmin are primarily $\alpha_{2}$-antiplasmin, and also $a_{2}$-macroglubulin and plasminogen activator inhibitor (PAI)-1 and $-2 .{ }^{49}$ Of these $a_{2}$-antiplasmin is the most potent inhibitor, but it is unable to inactivate plasmin that is bound to fibrin. A typical protein, bridging coagulation and fibrinolysis is thrombin activatable fibrinolysis inhibitor. Whilst it does not affect plasmin activity, it abrogates the fibrin cofactor function by proteolysis of the lysine residues needed for plasminogen and tPA to bind to fibrin. ${ }^{50}$

Platelets have been proposed to interfere in the fibrinolytic process by secreting proteins like plasminogen, PAl-1, $\alpha_{2}$-antiplasmin and $\alpha_{2}$-macroglobulin. ${ }^{11}$ However, the exact role by which platelets regulate plasmin activity and thereby fibrinolysis is still unclear. In addition, little is known about the impact of blood flow on the fibrinolytic process.

\section{Multiple interactions of platelets and coagulation in thrombus formation}

Platelets regulate and control the coagulation process in various ways.$^{41}$ The most well studied mechanism, as described above, is the exposure of phosphatidylserine on highly activated platelets, which triggers the assembly of several coagulation factors on the platelet surface, such as in the prothrombinase complex..$^{2}$ Platelet activation-induced phosphatidylserine exposure is absent in Scott patients, who are 
characterised by a diminished platelet-dependent thrombin generation and fibrin formation. ${ }^{53}$ Another way by which platelets control the coagulation process is by secreting pro- and anti-coagulant proteins that are stored in the alpha ( $\alpha$ )-granules. Recent analysis of the platelet secretome indicates that these proteins include fibrinogen, TFPI, VWF and factor V. As mentioned, platelets also secrete several proteins that influence the fibrinolytic system. In addition, it has been proposed that platelets form and express small amounts of tissue factor, however this is greatly debated. ${ }^{54}$ Also, to which extent this tissue factor is active in supporting coagulation is controversial, for instance given the much higher amounts of TFPI present in platelets. ${ }^{41}$ Furthermore, platelets control coagulation by causing contraction of a fibrin clot as a final step in stopping the bleeding. ${ }^{55}$ Integrin $\alpha_{11 b} \beta_{3}$ signalling is generally considered as a requirement for this clot contraction. ${ }^{56}$

\section{Aims and outline of this thesis}

In the present thesis, it is aimed to determine how platelet activation is linked to the dynamic processes of fibrin clot formation and fibrinolysis. Chapter 1, as a general introduction, is followed by an extensive review in Chapter 2 on the main platelet receptors (including: glycoprotein Ib-V-IX binding VWF, integrin $a_{1 \mathrm{lb}} \beta_{3}$ binding fibrinogen and glycoprotein VI binding collagen) and the signalling pathways evoked by these receptors that play a role in collagen-dependent thrombus formation under flow conditions. Discussed is how these receptors are used as molecular targets to prevent cardiovascular disease, and how deficiency or blockage of these receptors can affect normal haemostasis. In Chapter 3 (phospho)proteomics is employed as a tool to reveal new proteins involved in the main platelet-inhibitory pathway via the Gs protein, adenylyl cyclase and the cyclic AMP-dependent protein kinase A. Platelets from patients with Albright hereditary osteodystrophy (AHO), who carry a mutation in the GNAS complex gene locus, leading to Gs hypofunction, are studied in this chapter, in order to elucidate the functional and molecular consequences of diminished activity of this pathway. Chapter 4 focusses on the interaction of platelets with collagen via VWF and describes the consequence of desmopressin treatment in patients with acquired bleeding, serving to trigger the release of VWF from endothelial cells. This work aims to determine how in these patients with bleeding after surgery, the desmopressin-induced release of VWF affects platelet activation and coagulant activity in whole blood under flow conditions.

A protein with a well-established role in platelet adhesion and thrombus formation is integrin $\alpha_{11 b} \beta_{3}$, but whether and how $\alpha_{11 b} \beta_{3}$ can contribute to plateletdependent coagulation was still unclear. In Chapter 5, the principal signalling pathways are investigated how integrin $\alpha_{11 b} \beta_{3}$ via outside-in signalling supports the procoagulant activity of platelets. Chapter 6 describes how immobilised collagen and tissue factor support, in flowing whole blood, the dynamic processes of platelet adhesion, aggregation and fibrin clot formation. Furthermore it is determined 
how these immobilised thrombogenic proteins modulate not only the thrombus phenotype, but also the elasticity of a thrombus. Subsequently, Chapter 7 aims to provide further insight into the role of the tenase complex, consisting of factors VIII and IX, in platelet dependent coagulation and fibrin formation under conditions of flow. In Chapter 8, it is examined how platelets can have a role in fibrinolysis under flow conditions. Emphasis is placed on the binding of plasminogen to platelets as well as fibrin, such in relation to the generation of platelet phosphatidylserine exposure. The most important novel findings of this thesis are critically discussed and related to the current literature in Chapter 9.

\section{References}

1. Monroe DM, Hoffman M. What does it take to make the perfect clot? Arterioscler Thromb Vasc Biol. 2006;26:41-48.

2. Broos K, Feys HB, De Meyer SF, Vanhoorelbeke K, Deckmyn H. Platelets at work in primary hemostasis. Blood Rev. 2011;25:155-167.

3. Sim D, Flaumenhaft R, Furie B, Furie B. Interactions of platelets, blood-borne tissue factor, and fibrin during arteriolar thrombus formation in vivo. Microcirculation. 2005;12:301-311.

4. Versteeg HH, Heemskerk JW, Levi M, Reitsma PH. New fundamentals in hemostasis. Physiol Rev. 2013;93:327-358.

5. Tan KT, Lip GY. Red vs white thrombi: treating the right clot is crucial. Arch Intern Med. 2003;163:25342535 .

6. Cosemans JM, Mattheij NJ, Angelillo-Scherrer A, Heemskerk JW. The effects of arterial flow on platelet activation, thrombus growth and stabilization. Cardiovasc Res. 2013;99:342-352.

7. Offermanns S. Activation of platelet function through $G$ protein-coupled receptors. Circ Res.2006;99:1293-1304.

8. Jackson SP. The growing complexity of platelet aggregation. Blood. 2007;109:5087-5095.

9. Gibbins JM. Platelet adhesion signalling and the regulation of thrombus formation. $J$ Cell Sci. 2004;117:3415-3425.

10. Stegner D, Nieswandt B. Platelet receptor signaling in thrombus formation. J Mol Med (Berl). 2011;89:109-121.

11. Andrews RK, Berndt MC. Bernard-Soulier syndrome: an update. Semin Thromb Hemost. 2013;39:656662.

12. Lillicrap D. Von Willebrand disease: phenotype versus genotype: deficiency versus disease. Thromb Res. 2007;120:S11-16.

13. Nieswandt B, Watson SP. Platelet-collagen interaction: is GPVI the central receptor? Blood. 2003;102:449-461.

14. Watson SP, Auger JM, McCarty OJ, Pearce AC. GPVI and integrin $\alpha_{11 b} \beta_{3}$ signaling in platelets. J Thromb Haemost. 2005;3:1752-1762.

15. Ruggeri ZM, Mendolicchio GL. Adhesion mechanisms in platelet function. Circ Res. 2007;100:16731685.

16. Auger JM, Kuijpers MJ, Senis YA, Watson SP, Heemskerk JW. Adhesion of human and mouse platelets to collagen under shear: a unifying model. FASEB J. 2005;19:825-827.

17. Herr $A B$, Farndale RW. Structural insights into the interactions between platelet receptors and fibrillar collagen. J Biol Chem. 2009;284:19781-19785.

18. Cosemans JM, Iserbyt BF, Deckmyn H, Heemskerk JW. Multiple pathways to switch platelet integrins on and off. J Thromb Haemost. 2008;6:1253-1261. 
19. Hodivala-Dilke KM, McHugh KP, Tsakiris DA, Rayburn H, Crowley D, Ullman-Cullere M, Ross FP, Coller BS, Teitelbaum S, Hynes RO. $\beta_{3}$-integrin-deficient mice are a model for Glanzmann thrombasthenia showing placental defects and reduced survival. J Clin Invest. 1999;103:229-238.

20. Suh TT, Holmback K, Jensen NJ, Daugherty CC, Small K, Simon DI, Potter S, Degen JL. Resolution of spontaneous bleeding events but failure of pregnancy in fibrinogen-deficient mice. Genes Dev. 1995;9:2020-2033.

21. Tronik-Le Roux D, Roullot V, Poujol C, Kortulewski T, Nurden P, Marguerie G. Thrombasthenic mice generated by replacement of the integrin $\alpha_{11 b}$ gene: demonstration that transcriptional activation of this megakaryocytic locus precedes lineage commitment. Blood. 2000;96:1399-1408.

22. Siehler S. Regulation of RhoGEF proteins by G12/13-coupled receptors. Br J Pharmacol. 2009;158:4149.

23. Raslan Z, Naseem KM. The control of blood platelets by CAMP signalling. Biochem Soc Trans. 2014;42:289-294.

24. Germain-Lee EL, Ding CL, Deng Z, Crane JL, Saji M, Ringel MD, Levine MA. Paternal imprinting of Gas in the human thyroid as the basis of TSH resistance in pseudohypoparathyroidism type 1 . Biochem Biophys Res Commun. 2002;296:67-72.

25. Liu J, Chen M, Deng C, Bourc'his D, Nealon JG, Erlichman B, Bestor TH, Weinstein LS. Identification of the control region for tissue-specific imprinting of the stimulatory $G$ protein a-subunit. Proc Natl Acad Sci U S A. 2005;102:5513-5518.

26. Mantovani G, Ballare E, Giammona E, Beck-Peccoz P, Spada A. The Gsa gene: predominant maternal origin of transcription in human thyroid gland and gonads. J Clin Endocrinol Metab. 2002;87:47364740.

27. Mantovani G. Pseudohypoparathyroidism: diagnosis and treatment. J Clin Endocrinol Metab. 2011;96:3020-3030.

28. Turan S, Bastepe M. The GNAS complex locus and human diseases associated with loss-of-function mutations or epimutations within this imprinted gene. Horm Res Paediatr. 2013;80:229-241.

29. Pagel O, Loroch S, Sickmann A, Zahedi RP. Current strategies and findings in clinically relevant post-translational modification-specific proteomics. Expert Rev Proteomics. 2015;12:235-253.

30. Solari FA, Dell'Aica M, Sickmann A, Zahedi RP. Why phosphoproteomics is still a challenge. Mol Biosyst. 2015;11:1487-1493.

31. Hemker HC, Al Dieri R, Béguin S. Thrombin generation assays: accruing clinical relevance. Curr Opin Hematol. 2005;11:170-175.

32. Halbmayer WM, Haushofer A, Schon R, Mannhalter C, Strohmer E, Baumgarten K, Fischer M. The prevalence of moderate and severe FXII (Hageman factor) deficiency among the normal population: evaluation of the incidence of FXII deficiency among 300 healthy blood donors. Thromb Haemost. 1994;71:68-72.

33. Van der Meijden PE, Munnix IC, Auger JM, Govers-Riemslag JW, Cosemans JM, Kuijpers MJ, Spronk HM, Watson SP, Renné T, Heemskerk JW. Dual role of collagen in factor XII-dependent thrombus formation. Blood. 2009;114:881-890.

34. Muller F, Mutch NJ, Schenk WA, Smith SA, Esterl L, Spronk HM, Schmidbauer S, Gahl WA, Morrissey $\mathrm{JH}$, Renné T. Platelet polyphosphates are proinflammatory and procoagulant mediators in vivo. Cell. 2009;139:1143-1156.

35. Hojima Y, Cochrane CG, Wiggins RC, Austen KF, Stevens RL. In vitro activation of the contact (Hageman factor) system of plasma by heparin and chondroitin sulfate E. Blood. 1984;63:14531459.

36. Kannemeier C, Shibamiya A, Nakazawa F, Trusheim H, Ruppert C, Markart P, Song Y, Tzima E, Kennerknecht E, Niepmann M, von Bruehl ML, Sedding D, Massberg S, Gunther A, Engelmann B, Preissner KT. Extracellular RNA constitutes a natural procoagulant cofactor in blood coagulation. Proc Natl Acad Sci U S A. 2007;104:6388-6393. 
37. Renné T, Schmaier AH, Nickel KF, Blomback M, Maas C. In vivo roles of factor XII. Blood. 2012;120:4296-4303.

38. Zwaal RF, Schroit AJ. Pathophysiologic implications of membrane phospholipid asymmetry in blood cells. Blood. 1997;89:1121-1132.

39. Weiss HJ, Sussman I, Hoyer LW. Stabilization of factor VIII in plasma by the von Willebrand factor. Studies on posttransfusion and dissociated factor VIII and in patients with von Willebrand's disease. J Clin Invest. 1977;60:390-404.

40. Mann KG, Krudysz-Amblo J, Butenas S. Tissue factor controversies. Thromb Res. 2012;129 Suppl 2:S5-7.

41. Heemskerk JW, Mattheij N, Cosemans JM. Platelet-based coagulation: different populations, different functions. J Thromb Haemost. 2013;11:2-11.

42. Brass LF. Thrombin and platelet activation. Chest. 2003;124:18s-25s.

43. Lord ST. Fibrinogen and fibrin: scaffold proteins in hemostasis. Curr Opin Hematol. 2007;14:236-241.

44. Souri M, Osaki T, Ichinose A. The non-catalytic B subunit of coagulation factor XIII accelerates fibrin cross-linking. J Biol Chem. 2015;290:12027-12039.

45. Cilia La Corte AL, Philippou H, Ariens RA. Role of fibrin structure in thrombosis and vascular disease. Adv Protein Chem Struct Biol. 2011;83:75-127.

46. Wolberg AS, Campbell RA. Thrombin generation, fibrin clot formation and hemostasis. Transfus Apher Sci. 2008;38:15-23.

47. Law RH, Abu-Ssaydeh D, Whisstock JC. New insights into the structure and function of the plasminogen/plasmin system. Curr Opin Struct Biol. 2013;23:836-841.

48. Cesarman-Maus G, Hajjar KA. Molecular mechanisms of fibrinolysis. Br J Haematol. 2005;129:307321.

49. Draxler DF, Medcalf RL. The fibrinolytic system-more than fibrinolysis? Transfus Med Rev. 2015;29:102-109.

50. Mosnier LO, Bouma BN. Regulation of fibrinolysis by thrombin activatable fibrinolysis inhibitor, an unstable carboxypeptidase $B$ that unites the pathways of coagulation and fibrinolysis. Arterioscler Thromb Vasc Biol. 2006;26:2445-2453.

51. Browder T, Folkman J, Pirie-Shepherd S. The hemostatic system as a regulator of angiogenesis. J Biol Chem. 2000;275:1521-1524.

52. Bevers EM, Williamson PL. Phospholipid scramblase: an update. FEBS Lett. 2010;584:2724-2730.

53. Wielders SJ, Broers J, Ten Cate H, Collins PW, Bevers EM, Lindhout T. Absence of platelet-dependent fibrin formation in a patient with Scott syndrome. Thromb Haemost. 2009;102:76-82.

54. Bouchard BA, Gissel MT, Whelihan MF, Mann KG, Butenas S. Platelets do not express the oxidized or reduced forms of tissue factor. Biochim Biophys Acta. 2014;1840:1188-1193.

55. Ehrlicher A, Hartwig JH. Cell mechanics: contracting to stiffness. Nat Mater. 2011;10:12-13.

56. Shcherbina A, Cooley J, Lutskiy MI, Benarafa C, Gilbert GE, Remold-O’Donnell E. WASP plays a novel role in regulating platelet responses dependent on $\alpha_{11 b} \beta_{3}$ integrin outside-in signalling. $\mathrm{Br} J$ Haematol. 2010;148:416-427. 



\section{CHAPTER 2}

Targeting platelet receptor function in thrombus formation: The risk of bleeding

Swieringa F, Kuijpers MJ, Heemskerk JW, Van der Meijden PE

Blood reviews. 2014;28:9-21 Reprinted with permission 


\begin{abstract}
In this review, we presume that the process of thrombus formation, as assessed in whole blood flow studies and in experimental (murine) thrombosis studies, reflects the platelet responses in human haemostasis and thrombosis. Following this concept, we give an up-to-date overview of the main platelet receptors and signalling pathways that contribute to thrombus formation and are used as targets in (pre)clinical intervention studies to prevent cardiovascular disease. Discussed are receptors for thrombin, thromboxane $A_{2}, A D P$, ATP, prostaglandins, von Willebrand factor, collagen, CLEC-2 ligand, fibrinogen and laminin. Sketched are the consequences of receptor deficiency or blockage for haemostasis and thrombosis in mouse and man. Recording of bleeding due to (congenital) platelet dysfunction or (acquired) antiplatelet treatment occurs according to different protocols, while common laboratory methods are used to determine platelet function.
\end{abstract}

\title{
1. Introduction
}

Platelets are essential for normal haemostasis by forming a primary plug or thrombus after vascular injury, thus preventing further blood loss. Quantitative or qualitative platelet defects explain a considerable part of spontaneous or induced abnormal bleeding events in the population. Prospective studies suggest that the prevalence of bleeding due to platelet defects is high and comparable to that of von Willebrand disease. ${ }^{1}$ Although the most severe platelet disorders are identified at childhood, the majority of patients with milder platelet disorders remain undiagnosed until excessive bleeding occurs after specific challenges, as in surgery or trauma. On the other hand, undesired platelet activation contributes to arterial thrombotic diseases, and antiplatelet medication is the common therapy for secondary prevention, as in cardiovascular disease and stroke. Risk of bleeding is a well-known side effect of this suppression of platelet activation.

The premise of this paper is that the process of thrombus formation, such as assessed in whole blood flow studies and in experimental (murine) thrombosis studies, mirrors the platelet responses that determine haemostasis and thrombosis. In this scenery, we aim to give an up-to-date overview of the main platelet receptors and signalling pathways that contribute to thrombus formation and are used as targets in (pre)clinical intervention studies to attack cardiovascular disease. Since platelet dysfunction and treatment with antiplatelet therapy may both lead to a higher bleeding risk, we also sketch the current views of assessment of normal haemostasis by bleeding scores and discuss current methods to measure platelet function impairment. 


\section{Platelet receptors, antagonists and thrombus formation}

\subsection{Thrombin receptors, PAR1, 3 and 4}

Thrombin, a short-living proteolytic enzyme generated from prothrombin by coagulation factor $\mathrm{Xa}$, is not only a strong platelet agonist, but also a main effector of the coagulation cascade, inducing fibrin clot formation. ${ }^{2}$ Thrombin is generated at phosphatidylserine-exposing membranes from the damaged vessel wall and highly activated platelets. ${ }^{3}$ Its formation and inactivation can precisely be measured in platelet-rich plasma or blood by thrombin generation assays. ${ }^{4}$ Antithrombin in plasma binds and inactivates thrombin, a process that is enhanced by heparins.

In human platelets, thrombin cleaves and activates the protease-activated receptors (PAR)1 and PAR4. In comparison to PAR4, the former displays a higher affinity to thrombin, transmitting signals at subnanomolar thrombin concentrations. Accordingly, PAR1 functions as the key thrombin receptor of human platelets, while PAR4 rather sustains the action of PAR1. ${ }^{7}$ Platelets do not express the factor Xa receptor, PAR2. Both expressed receptors, PAR1 and 4, signal via the G-proteins G12/13a

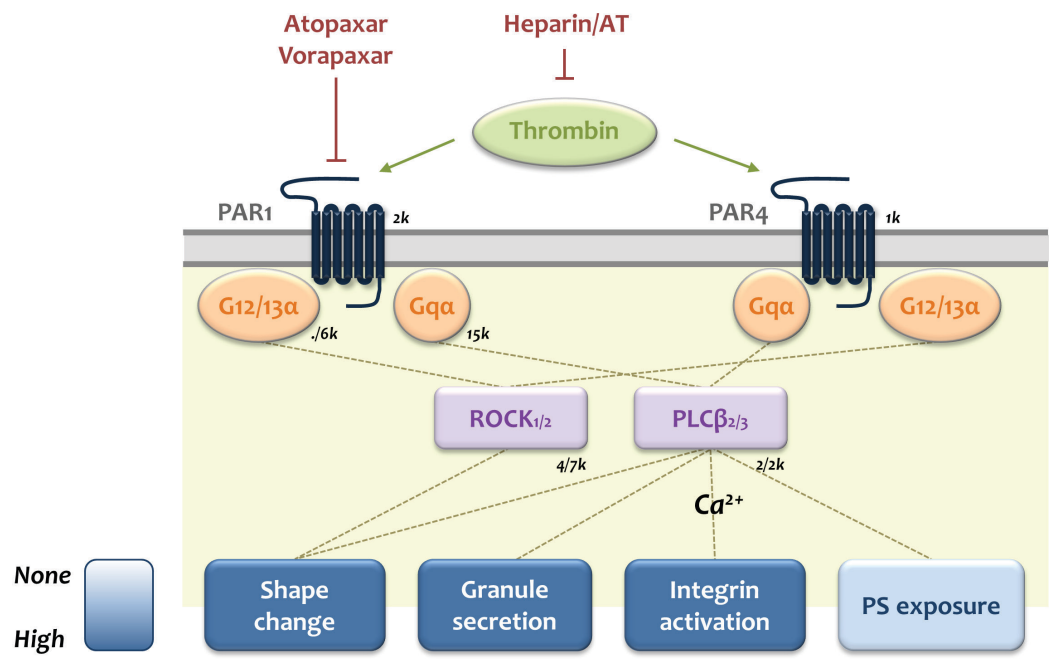

Figure 1. Signalling and intervention via the protease-activated receptors (PARs) for thrombin. Shown are established agonists and receptors on human platelets that are involved in normal haemostasis and are (potential) targets of antithrombotic treatment. Only key signalling proteins are indicated, as far as they associate with the receptors and act as essential molecular switches or second messengergenerating proteins (isoforms in smaller font). Asterisks point to the known presence of dysfunctional mutations in man. Numbers in italic refer to copy numbers $(\times 1,000)$ per platelet, as described in handbooks and proteomic analyses. ${ }^{5,6}$ For instance, for G12/13a the indication ./6k indicates expression levels of 'unknown' (G12a) and 6,000 (G13a) copy numbers per platelet. Dashed lines show networks of signal transmitting proteins linking to the indicated platelet responses (lower boxes). Activation strength of specific responses is represented by a heat map with colour codes from white to blue. Pharmacological inhibitors used in clinic or laboratory are indicated in red, physiological agonists in green. Abbreviations: AT, antithrombin; Gq, GTP-binding protein Gq; PLC, phospholipase C; PS, phosphatidylserine; ROCK, Rhoassociated protein kinase. 
and $\mathrm{Gq}$, which evoke the majority of functional platelet responses (Fig. 1). Current view is that both thrombin receptors only indirectly signal via Gia, i.e. through ADP secretion and autocrine effects. ${ }^{8}$ The PAR-induced activation of G12/13a results in platelet shape change by activation of Rho-associated protein kinase (ROCK) followed by actin cytoskeletal changes, whereas the activation of Gqa results in $\mathrm{Ca}^{2+}$-dependent integrin $\alpha_{11 b} \beta_{3}$ activation and secretion via the effector enzyme, phospholipase $C \beta$ (PLC $\beta$ ). Thrombin is a strong platelet agonist, which via PAR1/4 evokes maximal shape change, secretion and integrin activation, but thrombin by itself has little effect on platelet procoagulant activity (Fig. 1). ${ }^{3}$ In vitro, the various PAR receptors can be activated by specific thrombin receptor-activating peptides (TRAP). Patients with mutations in the genes encoding for PAR receptors have not yet been described, but a patient is reported with platelet Gqa deficiency suffering from mucocutaneous bleeding. ${ }^{9}$

Mouse platelets are devoid of PAR1 but express the isoform PAR3, which serves as a thrombin-binding co-factor for PAR4, promoting the activity of this receptor. ${ }^{10}$ Studies with Par3 $^{-1}$ and Par4 ${ }^{-1}$ mice revealed marked protection in experimental arterial thrombosis, which was associated with prolonged bleeding times upon challenge. ${ }^{11}$ In Par $4^{-1-}$ mice, thrombus formation in vivo was reduced compared to wild-types, but knockout platelets showed normal adhesion and normal support of fibrin deposition. ${ }^{12}$ These in vivo observations suggest a beneficial effect of blocking thrombin receptors in platelets.

In accordance with a key role of PAR1 in human platelet activation, clinical trials have been performed with PAR1 antagonists like Vorapaxar and Atopaxar. Vorapaxar has been evaluated in two phase III clinical trials. The TRACER study did not reveal superiority of Vorapaxar over standard therapy in the primary endpoint, which was a composite of death from cardiovascular causes, myocardial infarction, stroke, recurrent ischemia with rehospitalisation, or urgent coronary revascularisation. ${ }^{13}$ In the TRA 2P-TIMI 50 study, where patients with prior stroke were excluded, Vorapaxar was superior to placebo on top of standard care. ${ }^{14,15}$ This benefit was at the expense of an increased risk of intracranial bleeding, which was observed in both studies. The other PAR1 antagonist, Atopaxar, has been tested in several phase II trials, showing similar outcomes as Vorapaxar in terms of safety and efficacy. ${ }^{16}$

One consideration for the clinical practice, when prescribing PAR1 antagonists in combination with other antiplatelet agents is that, although an extra bleeding risk would be acceptable in comparison to gained antithrombotic protection, the patients need to take even more medication at extra costs, with lower compliance as a side effect. $^{17}$

\subsection{Thromboxane-prostanoid receptor, $T P$}

The TP receptor (one gene product, previously split into $\alpha$ and $\beta$ forms) is activated by the fatty acid derivative, thromboxane $A_{2} \cdot{ }^{18}$ This prostanoid is released from 
activated platelets as a very unstable metabolite, hence providing a rapid shut-off action mechanism upon stimulation of the TP receptors. Thromboxane $A_{2}$ formation requires the release of arachidonic acid from membrane phospholipids, a process catalysed by the $\mathrm{Ca}^{2+}$-dependent cytosolic phospholipase $\mathrm{A}_{2}$. Arachidonate acts as a substrate for cyclooxygenase 1 ( $C O X 1$ ) to produce prostaglandin $\mathrm{H}_{2}$, which is converted by thromboxane synthase into thromboxane $A_{2}$. Signalling via the TP receptor takes place via G12/13a and Gqa, similarly as for thrombin, but to a lower extent (Fig. 2). ${ }^{19}$ Activation of G12/13a again triggers platelet shape change via ROCK activation, while the low activation of Gqa/PLC $\beta$ is still sufficient for integrin activation and secretion.

In the laboratory, the stable thromboxane analogue U46619 is used to specifically trigger TP receptors. By itself, $U_{46619}$ is a weak agonist evoking limited functional responses, but it enhances the effects of other platelet agonists. In agreement with this, collagen-induced platelet activation relies for a considerable extent on the release of thromboxane $A_{2}$ and $A D P$, and ensuing TP and $P_{2} Y_{12}$ receptor activation, respectively. ${ }^{20}$ The few patients described with mutations in the thromboxane receptor experience mild bleeding. ${ }^{9}$ This agrees with the finding that also in $\mathrm{Tp}^{-/}$mice bleeding times are prolonged. ${ }^{21}$

The COX 1 complex is irreversibly inhibited by Aspirin and other non-steroid anti-inflammatory drugs (NSAIDs), such as Indomethacin, Diclofenac, Ibuprofen and Naproxen (Fig. 2). The classical test to check for inhibited COX1 activity is measurement of arachidonic acid-induced (i.e., thromboxane-dependent) platelet aggregation. Benefit of Aspirin in the treatment and secondary prevention of cardiovascular disease has clearly been shown in early clinical trials. ${ }^{22}{ }^{23}$ However, Aspirin has side effects experienced by some patients, particularly renal insufficiency, gastrointestinal symptoms and haemorrhagic complications. ${ }^{24}$ In accordance with this, also patients

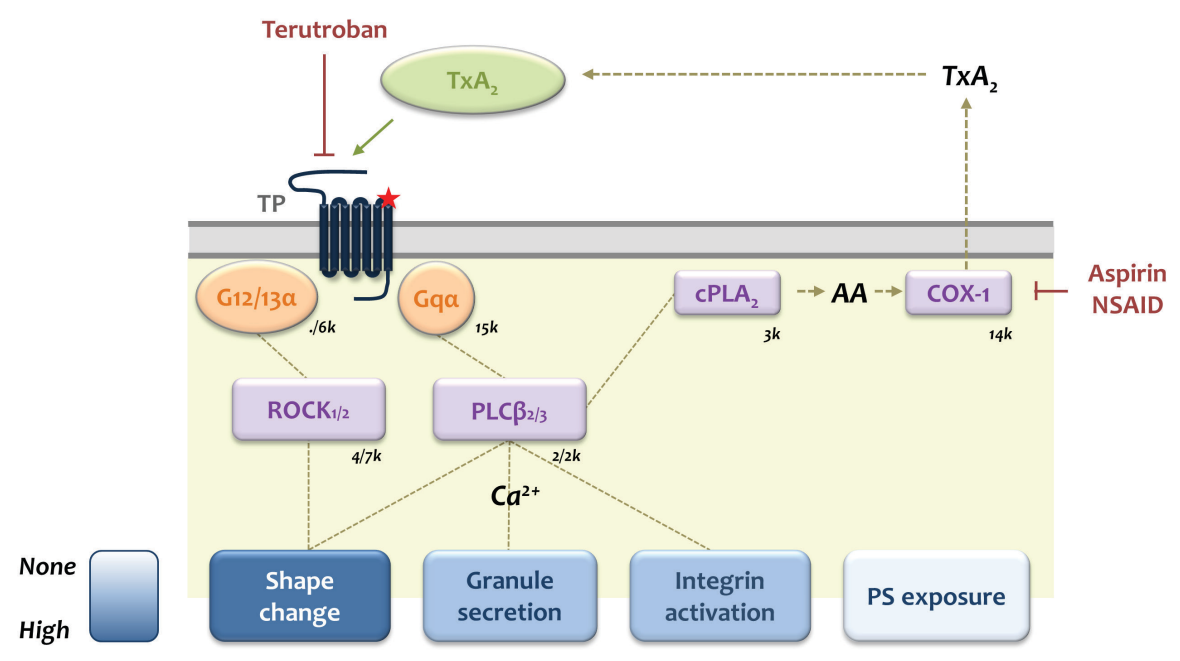

Figure 2. Signalling and intervention via the TP receptor for thromboxane $A_{2}$. For explanation, see Figure 1; AA, arachidonic acid; COX-1, cyclooxygenase 1; $\mathrm{CPLA}_{2}$, cytosolic phospholipase $\mathrm{A}_{2}$. 
with an inherited 'Aspirin-like' platelet defect frequently have a mild bleeding phenotype. ${ }^{9}$ While biochemical 'resistance' to Aspirin intake (i.e. lack of inhibition of thromboxane $A_{2}$ formation) is relatively rare, the drug prevents only part of the thrombotic events, ${ }^{25}$ likely because thromboxane is a weak platelet agonist. Intake of other NSAIDs may increase the cardiovascular risk, indicating that such drugs also affect other processes than platelet thromboxane formation. ${ }^{26}$

One of the developed antagonists for thromboxane receptors, Terutroban, has been tested clinically as an orally active drug. The TAIPAD study indicated that it was as effective as Aspirin in secondary prevention of thrombotic events in cardiovascular disease. ${ }^{27}$ On the other hand, superiority of Terutroban over Aspirin could not be demonstrated in the PERFORM trial, in preventing arterial events in patients with stroke. ${ }^{28}$

\subsection{Purinergic receptors, $P_{2} Y_{1}, P_{2} Y_{12}$ and $P_{2} X_{1}$}

The platelet dense granules ( $\delta$-granules) contain high concentrations of the adenosine nucleotides ADP, ATP and adenosine polyphosphate. In response to physiological agonists, these nucleotides are secreted and enforce platelet activation processes in an autocrine manner. Secreted ADP interacts with the purinergic $P_{2} Y_{1}$ and $P_{2} Y_{12}$ receptors, while ATP binds to $P_{2} X_{1}$ receptors (Fig. 3 ). The receptors $P_{2} Y_{1}$ and $P_{2} Y_{12}$ are coupled to the G-proteins, Gqa and Gia, respectively. ${ }^{29}$ Interaction of ADP with $\mathrm{P}_{2} \mathrm{Y}_{1}$ results in $\mathrm{Ca}^{2+}$ mobilisation, shape change and initial platelet aggregation through $\mathrm{PLC} \beta$ stimulation..$^{30}$ Stimulation of the other receptor, $\mathrm{P}_{2} \mathrm{Y}_{12}$, promotes the formation of large and stable platelet aggregates. ${ }^{31}$ The principal signalling mechanism of $\mathrm{P}_{2} \mathrm{Y}_{12}$ is by Gia-dependent stimulation of phosphoinositide 3-kinase ( $\mathrm{PI} / 3 \mathrm{~K} \beta / \gamma)$, which regulates platelet aggregation via actin cytoskeleton-dependent integrin $\alpha_{111} \beta_{3}$ activation. ${ }^{32}$ The alternative pathway of Gia-mediated inhibition of adenylate cyclase is nowadays considered to be of lesser importance. ${ }^{33}$ Under flow, continuous signalling via $\mathrm{P}_{2} \mathrm{Y}_{12}$ is required to maintain $\alpha_{11 b} \beta_{3}$ in the active conformation, and to ensure thrombus stability. ${ }^{34}$

Patients with $\mathrm{P}_{2} \mathrm{Y}_{1}$ deficiencies are not yet known. However, mice lacking this receptor on platelets are protected from collagen/epinephrine-induced thromboembolism and have a bleeding phenotype. ${ }^{30,35}$ The $\mathrm{P}_{2} \mathrm{Y}_{1}$-deficient platelets show reduced aggregation to all agonists. Conversely, mice with platelets overexpressing $\mathrm{P}_{2} \mathrm{Y}_{1}$ are more susceptible to thromboembolism and arterial thrombosis. ${ }^{36}$

Thirteen patients, experiencing a mild bleeding diathesis, have been described who either lack $\mathrm{P}_{2} \mathrm{Y}_{12}$ receptors or have a receptor mutation interfering with ligand binding. ${ }^{31,37}$ Platelets from these patients display a lesser and reversible aggregation in response to ADP, but have normal shape change and $\mathrm{Ca}^{2+}$ mobilisation. Bleeding is also reported for subjects with heterozygous point mutations in the receptor locus. ${ }^{38}$ Interestingly, reduced expression in platelets of the $\mathrm{P}_{2} \mathrm{Y}_{12}$-transmitting protein Gia 


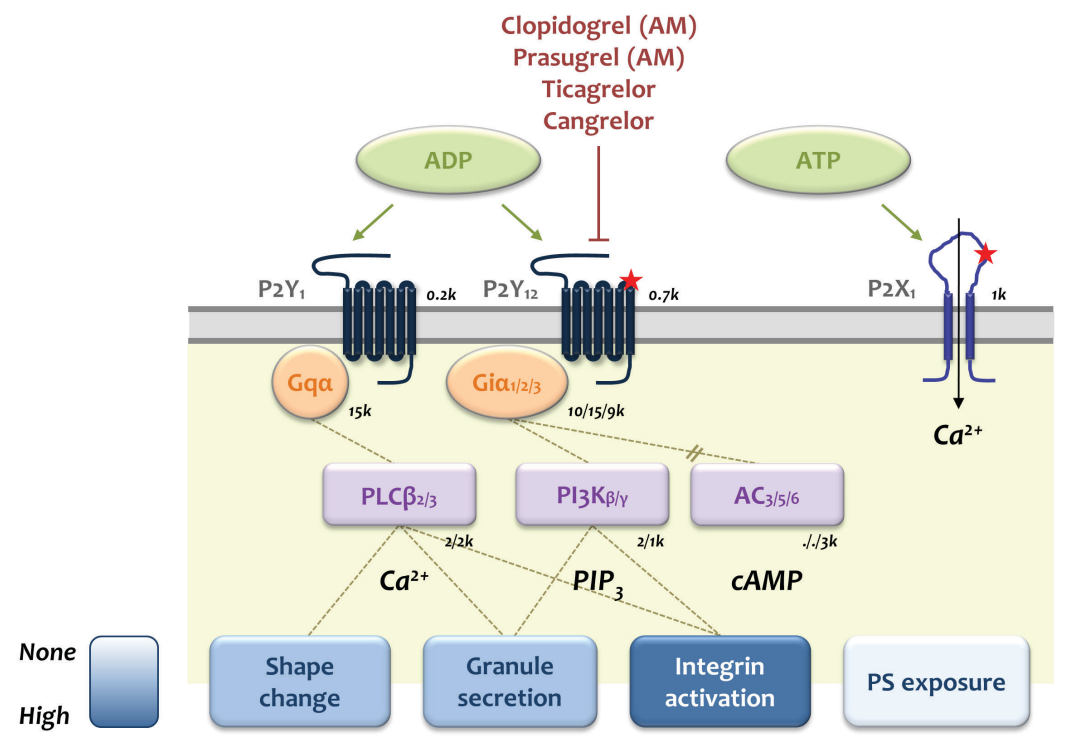

Figure 3. Signalling and intervention via the purinergic receptors for ADP and ATP. For explanation, see Figure 1; AC, adenylate cyclase; Clopidogrel (AM), Clopidogrel active metabolite; PIP $_{3}$, phosphatidylinositol 3-phosphate.

gives a similar phenotype 9 . Also mice deficient in $\mathrm{P}_{2} \mathrm{Y}_{12}$ display defective ADP-induced platelet aggregation and increased bleeding times. In vivo, their platelets form small and unstable thrombi. ${ }^{39}$ Conversely, transgenic mice expressing constitutively active $\mathrm{P}_{2} \mathrm{Y}_{12}$ exhibit increased platelet activation and arterial thrombosis. ${ }^{40}$

As reviewed elsewhere, $\mathrm{P}_{2} \mathrm{Y}_{12}$ is the pharmacological target of a number of common antiplatelet agents. ${ }^{31}$ Thienopyridines derived from the prodrugs Clopidogrel and Prasugrel inhibit $\mathrm{P}_{2} \mathrm{Y}_{12}$ in an irreversible way, whereas the drugs Ticagrelor and Cangrelor act as reversible $\mathrm{P}_{2} \mathrm{Y}_{12}$ inhibitors. When taken orally, Clopidogrel and Prasugrel need to be converted by hepatic cytochrome P450 enzymes into active, unstable metabolites. Despite well proven efficacy in multiple clinical trials, the use of Clopidogrel has certain drawbacks. ${ }^{31}$ The antiplatelet effect is delayed by the metabolic conversion of the prodrug, while its irreversible mode of action can be a problem if patients acutely need surgery. Furthermore, Clopidogrel has considerable inter-individual variability in responsiveness, which is clinically relevant since 'poor' responders may not be adequately protected from adverse cardiovascular events. ${ }^{41}$ Poor responsiveness to Clopidogrel is associated with loss-of-function mutations of cytochrome $\mathrm{P} 450$ is oforms. ${ }^{42}$ This variability in Clopidogrel responsiveness would argue for routine testing of platelet function for optimal care of patients, ${ }^{31}$ as suggested in a proof-of-concept trial. ${ }^{43}$ The structural analogue Prasugrel is less critically dependent on cytochrome $\mathrm{P} 450$ enzymes for conversion into the active metabolite in comparison to Clopidogrel. Prasugrel intake leads to faster appearance of the active metabolite in the blood, and overall greater inhibition of $\mathrm{P}_{2} \mathrm{Y}_{12}$-dependent platelet functions 
with less intra-individual variation. ${ }^{31,}{ }^{42}$ For both drugs, though, adequate dosing is important, as full $\mathrm{P}_{2} \mathrm{Y}_{12}$ receptor blockage causes a high bleeding risk.

The PLATO trial demonstrated that the drug Ticagrelor, which acts as an oral and reversible $\mathrm{P}_{2} \mathrm{Y}_{12}$ antagonist, induces a more consistent platelet inhibition and a greater antithrombotic efficacy, when compared to Clopidogrel, but still at the expense of bleeding. ${ }^{44}$ Another reversible $\mathrm{P}_{2} \mathrm{Y}_{12}$ inhibitor in use is the drug Cangrelor, which is administrated intravenously. A recent meta-analysis suggests that Cangrelor and Clopidogrel are similarly effective in reducing ischemic endpoints after percutaneous coronary intervention. ${ }^{45}$

The ATP receptor $\mathrm{P}_{2} \mathrm{X}_{1}$ operates as a cation channel that mediates transient $\mathrm{Ca}^{2+}$ influx through the plasma membrane and contributes to platelet activation by stimulating shape change (Fig. 3). The $\mathrm{Ca}^{2+}$ signal evoked by $\mathrm{P}_{2} \mathrm{X}_{1}$ synergises with that of other receptors, thus explaining why $\mathrm{P}_{2} \mathrm{X}_{1}$ stimulation enhances platelet responses evoked by other agonists. ${ }^{46}$ One case of a congenital deficiency in $\mathrm{P} 2 \mathrm{X}_{1}$ activation has been presented that was accompanied by a bleeding diathesis. ${ }^{47}$ Mice lacking $\mathrm{P}_{2} \mathrm{X}_{1}$ are viable, yet males are infertile. ${ }^{48,49} \mathrm{P}_{2} \mathrm{X}_{1}$-deficient mice show impaired platelet aggregation at low agonist doses, decreased thrombus formation in vivo and protection from thromboembolism. ${ }^{48}$ No specific drugs for clinical use have been described.

\subsection{Prostaglandin receptors, IP and EP1-4}

Prostaglandin $I_{2}$ (prostacyclin) and prostaglandin $E$ are prostanoids involved in haemostasis by interacting with the platelet IP and EP receptors, respectively. Next to nitric oxide, prostaglandin $I_{2}$ is one of the major endothelium-derived platelet inhibitors. It abrogates platelet activation via the Gsa-coupled IP receptors..$^{50}$ The signal pathway involves activation of the enzyme adenylyl cyclase, which raises the second messenger cyclic AMP and leads to protein phosphorylation events that are strongly inhibitory for platelets (Fig. 4). Several proteins integrate the inhibitory activities of cyclic AMP, in particular the G-protein regulators CalDAG-GEFI and RGS18.51, 52 Mice lacking the IP receptor display an increased tendency to arterial thrombosis..$^{53}$ For the human IP receptor, dysfunctional mutations are known resulting in a defective function or lack of expression, which were more frequently accompanied by coronary artery obstruction, when compared to silent mutations. ${ }^{54}$ Drugs targeting the IP receptor, such as Selexipag, llomedine and Teprostinil have primarily been tested for the treatment of pulmonary hypertension, but will also inhibit platelet function.

The vasodilatating prostaglandin $\mathrm{E}_{2}$ (as drug termed Dinoprostone) is released by various nuclear cells including macrophages. The mechanism by which prostaglandin $\mathrm{E}_{2}$ influences platelets is complex: it enhances platelet aggregation at low concentrations but inhibits at higher doses. The proaggregatory effect is mediated by prostaglandin $E_{2}$ binding to the EP3 receptors and a Gia-linked mechanism (Fig. $4),{ }^{55}$ similarly as described for ADP. It is suggested that EP3 signalling in this way 


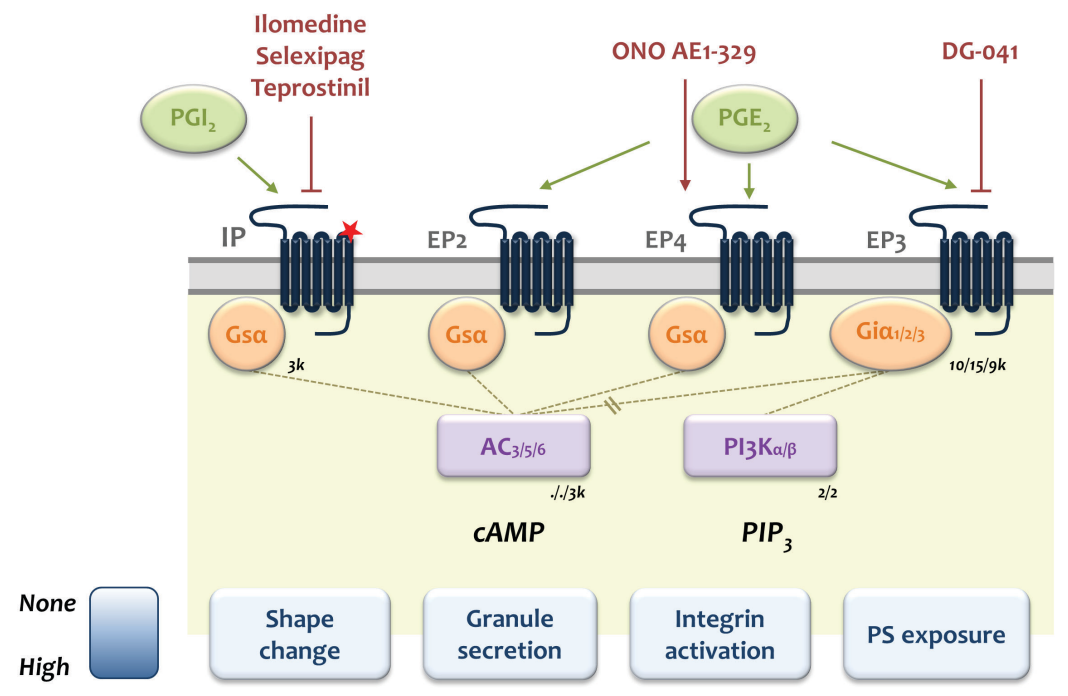

Figure 4. Signalling and intervention via the prostaglandin IP and EP receptors. For explanation, see Fig 1; PG, prostaglandin.

contributes to ADP- and collagen-induced platelet aggregation at low agonist doses. Functional mutations in the human $\mathrm{EP}_{3}$ gene have not been described. Mice lacking this receptor display a markedly decreased susceptibility to prostaglandin $\mathrm{E}_{2}$ promoted thrombosis.

At higher concentrations, prostaglandin $E_{2}$ binds to the EP1, EP2 and EP4 receptors. ${ }^{56}$ All of these are Gsa-coupled receptors, and thus inhibit platelets by increasing cyclic AMP levels. ${ }^{55}$ In human platelets, especially the EP4 receptor transmits the inhibitory effect of prostaglandin $E_{2}$. A selective EP4 agonist such as ONO AE1-329 thus suppresses human platelet activation on collagen..$^{57}$ It was proposed that EP4 agonists can serve as antithrombotics, e.g. in cases where Aspirin and ADP antagonists alone are insufficient to prevent thrombotic events.

The EP3 receptor is of some interest as a target, e.g. in atherothrombotic disease where prostaglandin $E_{2}$ levels are increased at sites of atherosclerotic lesions. ${ }^{58,59} \mathrm{An}$ EP3 antagonist at the beginning of clinical development (phase II) is DG-041. Ex vivo, this compound inhibits the potentiating effects of prostaglandin $E_{2}$ on rat and human platelet aggregation induced by various agonists, in rat without increasing bleeding times. ${ }^{59,60}$

\subsection{Von Willebrand factor receptor, GPIb-V-IX}

Von Willebrand factor (VWF) is a large glycoprotein that is released from endothelial cells in blood plasma as well as from platelets. Ultralarge multimers (strings) of VWF freshly secreted by endothelial cells are most activating for platelets. Under normal conditions these strings are cleaved by the protease ADAMTS13, but when 


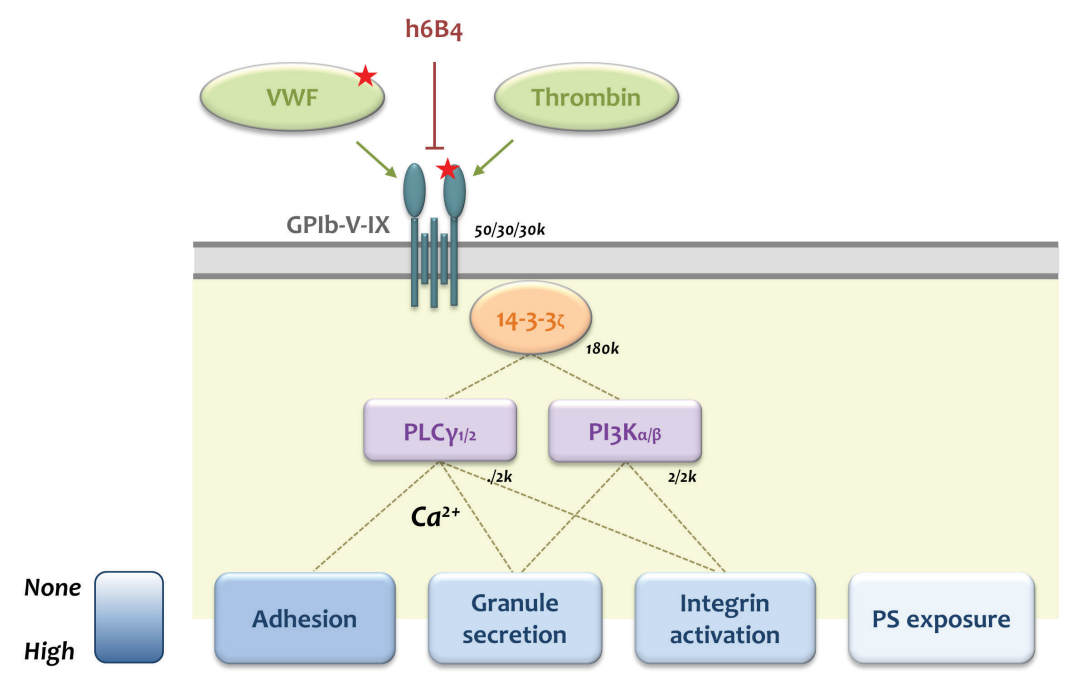

Figure 5. Signalling and intervention via the von Willebrand factor (VWF) receptor, GPIb-V-IX. For explanation, see Figure 1.

ADAMTS13 activity is absent they become clinically problematic causing thrombotic thrombocytopenia purpurea (TTP). ${ }^{61}$ Bleeding in TTP patients is considered to be a consequence of VWF-induced platelet agglutination and thrombocytopenia.

In healthy subjects VWF circulates as intermediate-size multimers, which capture factor VIII and bind to subendothelial matrix components (collagen, laminin) and platelet aggregates. Under high shear stress conditions as in the arterial circulation, the VWF multimers change in conformation and avidly interact with the platelet GPIbV-IX complex, forming a so-called catch bond. ${ }^{62}$ The consequence is transient platelet adhesion which, in the presence of other receptor-ligand interactions (e.g. collagen), turns into firm adhesion and platelet activation under flow. ${ }^{63,64}$ In the presence of high shear gradients, for instance at atherosclerotic geometries, platelets can form thrombi at post-stenotic sites in a GPIb-dependent manner with limited activation. ${ }^{65}$, 66

In the laboratory, VWF-GPIb interaction is induced by the venom components ristocetin and botrocetin, which force VWF to bind to GPIb-V-IX in the absence of shear. Ristocetin- or shear-induced VWF-GPIb interaction leads to PLC $\gamma$ and PI3K activity and, thereby, activated $\alpha_{11 b} \beta_{3}{ }^{67}$ The GPIb-associated adaptor protein $14-3-3 \zeta$ plays an intermediary role in assembly of the concerning signal complex. ${ }^{68}$ The binding of VWF to GPIb-V-IX (under shear) induces only limited signalling events (Fig. 5). However, it enforces multiple amplification pathways in platelets, including thromboxane $\mathrm{A}_{2}$, ADP and phospholipase $D$ pathways. ${ }^{68,69}$

There is increasing interest in the further roles of VWF and GPIb-V-IX under conditions of coagulation and low shear, as in venous thrombosis. An initial observation was that thrombin binding to GPIb accelerates platelet activation via 
PAR1. ${ }^{70}$ Several other coagulation factors bind to the GPIb-V-IX complex as well, but the functional consequences are not well understood. ${ }^{3}$ Under coagulant conditions, VWF binding to GPIb stimulates platelet procoagulant activity and the formation of fibrin fibres on human and mouse platelets. ${ }^{71}$ This is compatible with the finding that in mice both VWF and GPIb contribute to venous thrombosis in vivo, although the precise mechanisms are not fully understood. ${ }^{72}, 73$ Together, this points to the existence of synergistic pathways of GPIb- and coagulation (thrombin?)-mediated platelet responses.

Genetic deficiency or dysfunction of platelet GPIb-V-IX leads to Bernard-Soulier syndrome, a rare bleeding disorder characterised by thrombocytopenia and giant platelets. ${ }^{9} \mathrm{~A}$ similar phenotype is observed in mice deficient in the GPIba or GPIb $\beta$ chains. ${ }^{74,75}$ Interestingly, also in mice, the GPV chain is considered to contribute to thrombus formation by acting as a cleavable substrate for thrombin. ${ }^{76}$ The VWFGPIb axis may also be a suitable target for pharmacological intervention. In baboons, the humanised anti-GPIb antibody h6B4 acts as a safe antithrombotic drug with minimal bleeding. ${ }^{77} \mathrm{~A}$ second approach to interfere with GPIb-mediated thrombus formation may be to target VWF. It has been shown that antibodies against the $A 3$ domain of VWF, through which GPIb interacts, inhibit arterial thrombus formation in vivo. $^{78}$

Bleeding is a recurrent phenomenon in patients with von Willebrand disease, a disorder characterised by quantitative or qualitative abnormalities in $\mathrm{VWF}^{79}$ In most forms of von Willebrand disease, ristocetin-induced platelet clumping via VWF (socalled platelet agglutination) is reduced, thus stressing the importance of VWF-GPIb binding for normal haemostasis. In platelet-type $2 \mathrm{M}$ and type $2 \mathrm{~B}$ von Willebrand disease, the VWF-GPIb interaction is increased leading to 'spontaneous' platelet aggregation and even thrombocytopenia. ${ }^{79}$

\subsection{Collagen receptor, GPVI}

The principal signalling receptor for collagen on platelets is glycoprotein VI (GPVI), a member of the immunoglobulin superfamily, which associates with the ITAMbearing FCR $\gamma$-chain. ${ }^{80} \mathrm{GPVI}$ is only expressed on platelets and megakaryocytes, and it requires dimerisation for binding to collagen. ${ }^{81}$ Stimulation via GPVI leads to full platelet activation, including integrin activation, secretion and procoagulant activity. ${ }^{82}$ Established ligands of GPVI are the fibrillar collagen types I or III, collagen-related peptides containing repeats of the sequence, glycineproline-hydroxyproline, and the snake venom convulxin.

The GPVI-induced signalling mechanism operates through protein tyrosine kinases, and resembles the signalling induced by various other adhesive receptors (Fig. 6). ${ }^{83,} 84$ In brief, ligand-occupied GPVI dimers form a large signalling complex, via activation of Src-family kinases, phosphorylation of the ITAM motif of the FCR $\gamma^{-}$ chain, and activation of the central tyrosine kinase, Syk. ${ }^{85}$ This results in a cascade of 
phosphorylation events, with as an end result the activation of key effector enzymes at the plasma membrane, $\mathrm{PLC} \gamma$ and $\mathrm{PI} 3 \mathrm{~K}$ isoforms. ${ }^{86,87}$ The functional consequences are prolonged $\mathrm{Ca}^{2+}$ mobilisation, secretion, integrin activation, procoagulant activity and membrane blebbing. ${ }^{3}$ The $\mathrm{Ca}^{2+}$-dependent proteins, protein kinase $\mathrm{C}$ and CalDAG$\mathrm{GEFI}$, regulate many of the downstream responses to secretion and integrin activation (see below). ${ }^{88,89}$

The interest in GPVI as a target for antithrombotic therapy was greatly raised by the recognition that mice lacking the $\mathrm{FCR} \gamma$-chain showed a greatly impaired thrombus formation in experimental models of arterial thrombosis, that was not accompanied by increased tail bleeding. ${ }^{\circ} \mathrm{A}$ similar phenotype was found after depletion of platelet GPVI by injection of antibodies against this receptor. ${ }^{91}$ In addition, blocking of GPVI impaired thrombus formation on atherosclerotic plaques, e.g. in a mouse model of acute plaque rupturing. ${ }^{22}$ On the other hand, deficiency in GPVI did not affect arterial thrombus formation in models of more severe vascular damage with tissue factor exposure, where thrombin generation is considered to be the major driving force of thrombosis. ${ }^{93,}, 94$

Several patients have been described with circulating anti-GPVI antibodies, accompanied by low expression of platelet GPVI, thrombocytopenia and a mild bleeding tendency ${ }^{80,95}$ Mild mucocutaneous bleeding symptoms have been reported for subjects with compound heterozygous mutations or a frame-shift mutation in the GP6 gene..$^{96}$ In addition, large scale genome-wide analyses indicate that variation in the GP6 locus is one of the major predictors in determining the extent of platelet activation. 97,98

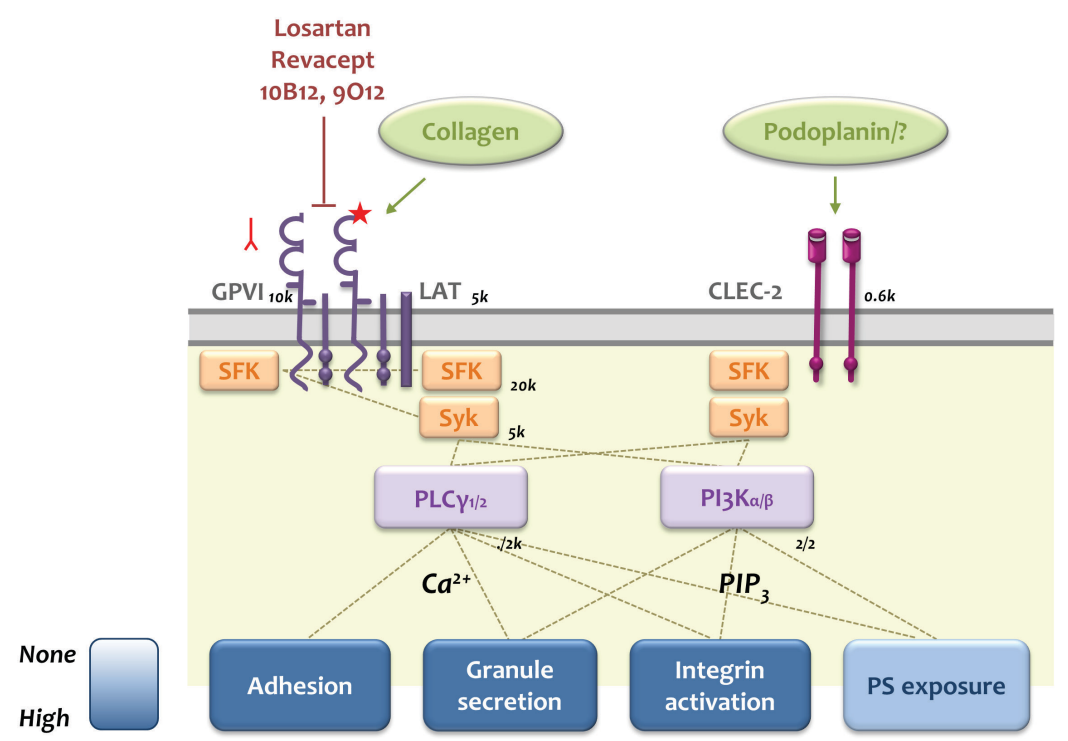

Figure 6. Signalling and intervention via the collagen receptor, GPVI, and the platelet CLEC-2 receptor. For explanation, see Figure 1; SFK, Src family kinases. 
In vitro perfusions with mouse blood indicated that thrombus formation on collagen relies on the presence of both FcRY-chain and GPVI. ${ }^{99}, 100$ Thrombus formation on collagen is greatly impaired in mice lacking proteins of the GPVI signalling complex, i.e. $\mathrm{PLC} \gamma$ or $\mathrm{PI} 3 \mathrm{~K}$ isoforms, ${ }^{86,101}$ whereas animals with constitutively active PLC $\gamma 2$ show aggravated thrombus formation ${ }^{102}$. These mouse studies jointly suggest that the major functional role of GPVI is the limiting of excessive blood loss upon injury with substantial collagen exposure. ${ }^{103}$

Since anti-GPVI agents have the potential to reduce arterial thrombus formation with limited effect on haemostatic activity, there have been several initiatives to develop such substances. Small GPVI-inhibiting molecules (e.g. Losartan) and blocking anti-GPVI antibodies (10B12, 9O12) have been generated for experimental use with animal and human blood. ${ }^{90}, 95$ Such anti-GPVI agents strongly inhibit thrombus formation on collagen under flow conditions. ${ }^{104,}{ }^{105}$ Injection of the anti-GPVI OM2Fab fragment into cynomolgus monkeys caused inhibition of collagen-induced platelet aggregation. ${ }^{106}$ In a different approach, a soluble dimeric GPVI-Fc fusion protein (Revacept) has been generated to block the GPVI-binding sites on exposed collagen, causing suppression of murine arterial thrombosis. ${ }^{107}$ This GPVI-FC fusion protein is presently tested in a phase III trial. ${ }^{108}$ Taken together, GPVI is an interesting therapeutic target that deserves further exploration.

\subsection{Novel receptor, CLEC-2}

The C-type lectin-like type II membrane glycoprotein CLEC-2 has recently been identified as a receptor that also can strongly activate platelets. Ligands of this receptor are the snake venom toxin rhodocytin and the glycoprotein podoplanin. ${ }^{109,}, 110$ The physiological ligand of CLEC-2 in the vascular system is still unclear. The signalling pathway evoked by CLEC-2 clustering is similar to that of GPVI, i.e. via activated protein tyrosine kinases resulting in PLC $\gamma$ activation, although in this case the mechanism does more rely on Syk than on Src kinases (Fig. 6). ${ }^{103,}, 10$ Phosphoproteomics analysis of activated platelets also points to a substantial, but not complete overlap of GPVI and CLEC-2 signalling events. ${ }^{111}$

In mice, conditional knockout of CLEC-2 or treatment with anti-CLEC-2 antibody caused reduced thrombus stability and prolonged tail bleeding times. ${ }^{10,}{ }^{112}$ Strikingly, combined in vivo depletion of GPVI and CLEC-2 by antibody treatment, or genetic deficiency of platelet GPVI plus CLEC-2 resulted in severe impairment of arterial thrombus formation, but at the expense of prolonged bleeding times. ${ }^{113}$ This points to a functional redundancy of the two receptors in thrombosis and haemostasis. Although there are only few human data available, together this suggests that CLEC-2 is an interesting therapeutic target, but bleeding might be an unavoidable side effect. 


\subsection{Fibrinogen receptor, integrin $\alpha_{11 b} b_{3}$}

Integrin $\alpha_{I I b} \beta_{3}$ (GPIIb/IIla) is the most abundantly expressed receptor on platelets. On resting platelets $\alpha_{11 b} \beta_{3}$ assumes a bent form with low ligand affinity. Upon platelet activation by most agonists, the integrin opens by a reversible conformational change, which increases its affinity for fibrinogen, fibronectin, vitronectin and VWF. This inside-out integrin signalling occurs via $\mathrm{Ca}^{2+}$-dependent activation of protein kinase $C$ or CaIDAG-GEFI, culminating in Rap1b activation and binding of the actin cytoskeletal proteins talin-1 and kindlin-3 to the cytoplasmic $\alpha_{1 \mathrm{Ib}} \beta_{3}$ domains (Fig. 7A). ${ }^{114-}$ ${ }^{116}$ In the activated state, $\alpha_{1 \mathrm{lb}} \beta_{3}$ supports platelet adhesion to fibrinogen and platelet aggregation. Ligand binding to $\alpha_{1 \mathrm{Ib}} \beta_{3}$ furthermore leads to a train of signalling events, mediated by Src and Syk protein tyrosine kinases, a process known as outside-in signalling (Fig. 7B). ${ }^{117}$ This process drives the spreading of platelets on fibrinogen, the retraction of fibrin clots, and the procoagulant activity of platelets. ${ }^{118}$

Mutations in the genes encoding for $\alpha_{11 b}$ and $\beta_{3}$ give rise to one of the most common platelet disorders, Glanzmann's thrombasthenia. Glanzmann patients have platelets lacking $\alpha_{11 b} \beta_{3}$ or with non-functional $\alpha_{l l b} \beta_{3}$, and characteristically show mucocutaneous and gastrointestinal bleeding symptoms as well as excessive trauma-related bleeding. ${ }^{119}$ Platelets from these patients show impaired aggregation, spreading and clot retraction. Mice lacking expression of the $\alpha_{1 \mathrm{lb}}$ or $\beta_{3}$-chain are greatly impaired in arterial thrombosis, and have a bleeding phenotype, with prolonged tail bleeding times and spontaneous haemorrhages. ${ }^{120,121}$

The autosomal recessive syndrome, leukocyte adhesion deficiency type III (LAD-III or LAD-1/variant), refers to a dysfunction of integrins $\beta_{1}, \beta_{2}$ and $\beta_{3}$ in platelets and leukocytes. While the integrin expression levels are normal, LAD-III patients experience severe bleeding and recurrent infections. Recently, it was established that this disorder is caused by mutations in the gene of kindlin-3, which result in abrogated integrin inside-out signalling. ${ }^{122,123}$ The bleeding phenotype is explained particularly, but not exclusively, by the inability of $\alpha_{11 b} \beta_{3}$-mediated platelet aggregation. ${ }^{9}$

Three inhibitors of $\alpha_{11 b} \beta_{3}$ are available for clinical use, namely Abciximab, Eptifibatide and Tirofiban. These drugs are given intravenously to patients undergoing percutaneous coronary intervention after acute coronary syndrome. Integrin inhibitors have been reported to cause more haemorrhagic events in women, but this might be due to improper weight-adjusted dosing. ${ }^{124}$ Cardiologists mostly use $a_{11 b} \beta_{3}$ inhibitors in high-risk patients not pretreated with $\mathrm{P}_{2} \mathrm{Y}_{12}$ blockers. ${ }^{125}$ In the past, also oral $\alpha_{1 \mathrm{lb}} \beta_{3}$ inhibitors have been tested, but clinical trials were cancelled due to increased mortality, likely due to a ligand-mimetic function of the compounds, provoking fibrinogen binding to $\alpha_{11 b} \beta_{3}{ }^{126}$ Summarising, given the fundamental role of $\alpha_{11 b} \beta_{3}$ in haemostasis, inhibition of this glycoprotein needs to be performed with great care. 
A

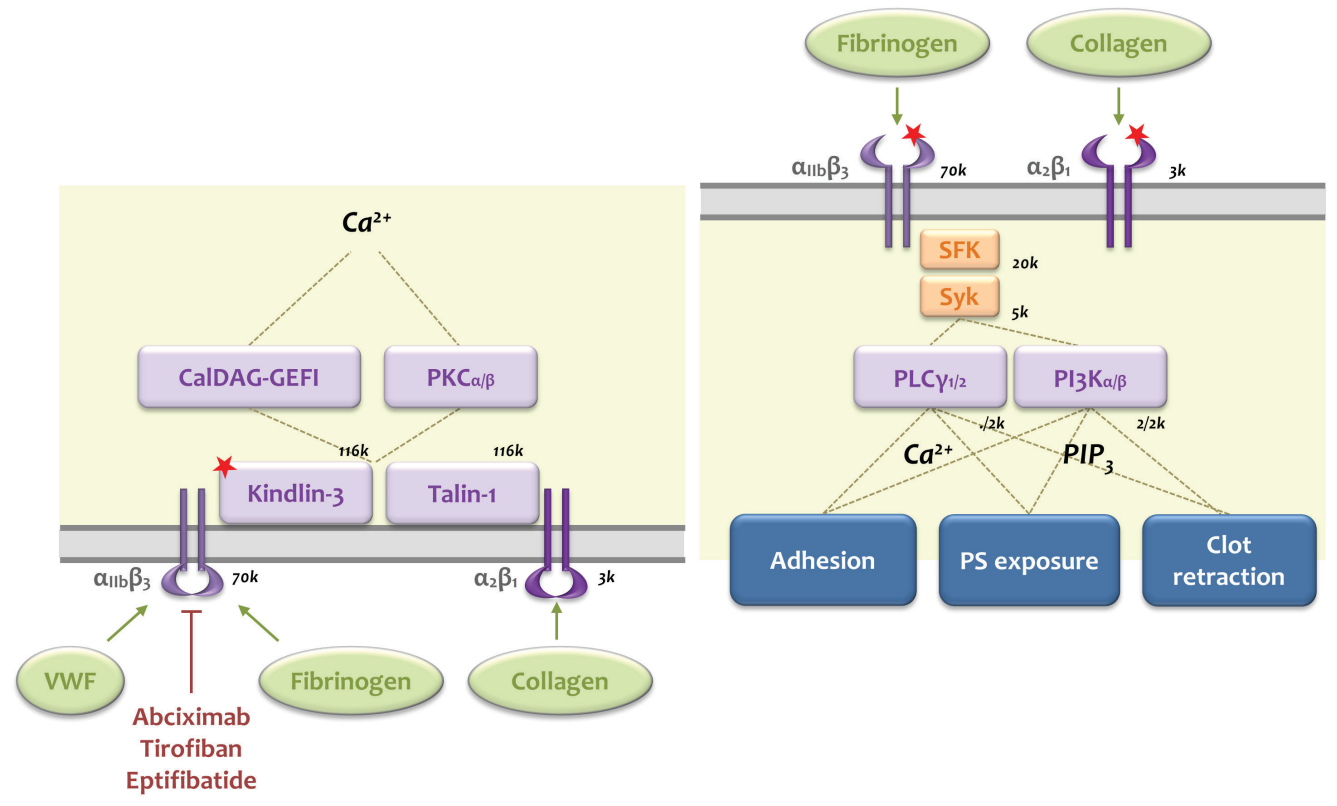

Figure 7. Signalling and intervention via platelet integrins. (A) Inside-out signalling, (B) outside-in signalling. For explanation, see Figure 1.

\subsection{Other platelet integrins}

Platelets also express other integrins than $\alpha_{11 b} \beta_{3}$, which serve as receptors for adhesive proteins. At least for integrin $\alpha_{2} \beta_{1}$, receptor function depends on conformational changes and leads to outside-in signalling events. Integrin $\alpha_{2} \beta_{1}$ (GPla/lla) serves as a receptor for fibrillar collagens. Its activation depends on cytoskeleton-mediated kindlin-3 and talin-1 interactions (Fig. 7A). ${ }^{115}$ Likely, lack of activation of $\alpha_{2} \beta_{1}$ explains why LAD-III patients (with defective kindlin-3) suffer from a more severe bleeding tendency than Glanzmann patients (missing only $\alpha_{11 b} \beta_{3}$ ). ${ }^{127}$

In mice, deficiency in $\alpha_{2} \beta_{1}$ results in limited impairment in arterial thrombus formation, which is detected as increased thrombus instability. ${ }^{128}$ Mice lacking $\alpha_{2}$ or $\beta_{1}$ show unchanged tail bleeding times. ${ }^{100,129}$ However, combined deficiency of GPVI and $\alpha_{2} \beta_{1}$ leads to a severe bleeding tendency, accompanying major defects in thrombus formation. ${ }^{130}$ This suggests that $\alpha_{2} \beta_{1}$ plays a supportive rather than essential role in the interaction between platelets and collagen, ${ }^{115}$ although recent evidence indicates that $\beta_{1}$ integrins do contribute to platelet activation processes. ${ }^{131}$ Two patients suffering from minor bleeding are described with reduced expression of $\alpha_{2} \beta_{1}$ and impaired platelet adhesion to collagen. ${ }^{132,133}$ However, whether the bleeding is due to the altered glycoprotein expression is unclear. ${ }^{80}$ In studies of thrombus formation with mouse and human blood, $\alpha_{2} \beta_{1}$ enforces the GPVI-dependent platelet adhesion 
to collagen and ensuing platelet activation. ${ }^{63}$ Taken together, $a_{2} \beta_{1}$ seems to have a dispensable but still relevant role in haemostasis and thrombosis.

Recently, also the platelet receptor for vascular laminins, integrin $\alpha_{6} \beta_{1}$, was found to play an important role in murine platelet activation and arterial thrombosis in vivo, ${ }^{134}$ suggesting that also this integrin could be a target of antithrombotic treatment.

\section{Thrombus formation - interactions of multiple platelet receptors}

Flow studies have gained considerable insight into the combinatory roles of various platelet ligands and receptors in the process of thrombus formation, reflecting those in haemostasis and arterial thrombosis. Current models of thrombus formation start with shear-dependent platelet adhesion to VWF via GPIb-V-IX followed by platelet activation via collagen, but also other platelet substrates will contribute to initial platelet adhesion. ${ }^{135}$ Platelet activation via GPVI, and likely also CLEC-2, initiates responses like shape change (pseudopod formation), $a / \delta$-granule secretion, thromboxane release and integrin activation which, together, evoke a train of autocrine stimulatory events to activate and trap flowing platelets into the growing thrombus. ${ }^{64,83}$ Thrombus formation is thus seen as a multifactorial event with amplification loops by receptor interactions of thrombin/PAR isoforms, $A D P / P 2 Y$ isoforms, ATP/P2 $X_{1}$, thromboxane $A_{2} / T P$ receptors and fibrinogen $/ \alpha_{11 b} \beta_{3}$ (Fig. 8). However, also many other ligand-receptor interactions - not discussed in this review - contribute to this complexity. These involve, for instance, contactdependent signalling processes, activation via a-granule secretion products (Gas6, thrombospondin), tyrosine phosphatase-linked receptors, and mechanisms controlling fibrin formation, thrombus contraction and thrombus stability. ${ }^{136-138}$ Moreover, these processes may occur at different loci in a growing thrombus. Below, we discuss platelet activation processes that are most relevant for haemostasis in man.

The combined involvement of multiple ligand-receptor combinations in thrombus formation becomes especially apparent, when more than one interaction is absent. This is the case for patients lacking $\alpha$ - or $\delta$-granules in platelets, often associated with bleeding symptoms of various severities. Patients with the rare Hermansky-Pudlak syndrome have mutations in one of nine HPS genes, resulting in complete $\delta$-granule and lysosome deficiency. ${ }^{9,} 139$ The functional defect of platelets is at least in part explained by impaired ADP and ATP secretion. More common is a partial impairment of $\delta$-granule secretion with unknown genetic causes. ${ }^{139}$ Abnormalities in the a-granules, which store a large variety of proteins, are seen in several syndromes. Well studied is the Gray platelet syndrome, a mild bleeding disorder characterised by absence of a-granules and accompanied with thrombocytopenia, due to mutations in the NBEAL2 gene. ${ }^{9,} 139$ Mice deficient in Nbeal2 phenocopy this syndrome and are protected from arterial thrombus formation; their platelets show diminished adhesion, aggregation and procoagulant activity. ${ }^{140}$ It is 


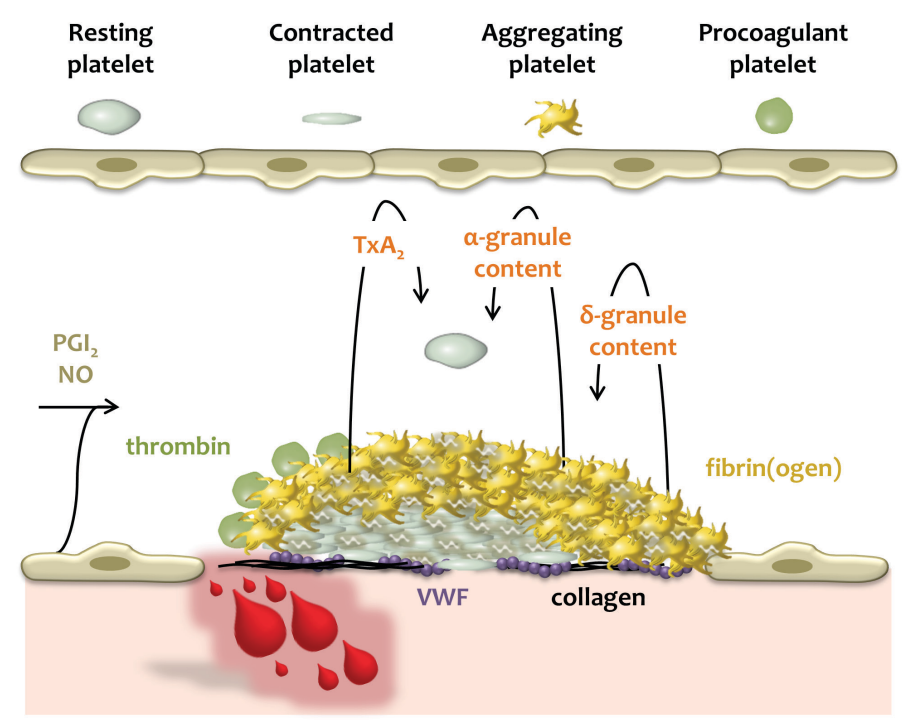

Figure 8. Simplified mechanism of platelet agonists and adhesive ligands in thrombus formation. See text.

still unclear which of the a-granule stored proteins mediate these platelet responses. Platelet cytoskeleton defects, such as May-Hegglin anomaly (MYH9 gene mutation) and Wiskott-Aldrich syndrome (WAS mutation) are accompanied by changes in platelet size and thrombocytopenia. ${ }^{9}$ Pharmacological interventions to inhibit platelet granule secretion or cytoskeletal changes are not known.

A few intracellular signalling proteins with major roles in platelet functions have been studied as potential targets for therapy. Well advanced are studies on pharmacological inhibition of $\mathrm{Pl} 3 \mathrm{~K}$, particularly of the $\beta$ isoform. In flow perfusion studies, $\mathrm{Pl} 3 \mathrm{Ka} / \beta$ blockers suppress collagen-induced $\mathrm{Ca}^{2+}$ mobilisation, secretion, integrin activation and platelet procoagulant activity ${ }^{86} \mathrm{~A}$ phase I study consisting of intravenous infusion of the PI3K $\beta$ blocker AZD6482 (an analogue of TGX-221) indicated a strong platelet-inhibiting effect ex vivo. ${ }^{141}$

\section{Bleeding and platelet dysfunction or inhibition}

\subsection{Predicting mild platelet disorders with questionnaires}

Typical bleeding symptoms that accompany quantitative or qualitative platelet disorders are excessive bruising, prolonged menses and abnormal bleeding after haemostatic challenges. The magnitude of bleeding depends on the type and severity of the platelet defect. ${ }^{1}$ Since patients with a platelet disorder often display only mild bleeding symptoms, which are also features of von Willebrand disease or coagulation deficiencies, proper diagnosis is not always easy. ${ }^{142}$ The complexity is well illustrated 
by the outcome of the UK GAPP study where, in subjects with suspected platelet function deficiencies and abnormal bleeding symptoms, the genetic causes of the signalling defects could only be determined in a minority of the subjects. ${ }^{20}$

In the clinic, there is ongoing interest in the development of bleeding assessment tools (BATs) for the diagnostic evaluation of haemorrhagic symptoms by way of a questionnaire and bleeding score. Most questions concern the family history of spontaneous and challenged bleeding events, as well as the frequency and severity of the bleeding symptoms. Specific BATs have been developed for von Willebrand disease, paediatric patients, and women with menorrhagia. ${ }^{143}$ In 2010 the ISTH published a consensus BAT to standardise the description of significant bleeding symptoms and the diagnosis of bleeding disorders with main focus on von Willebrand disease. ${ }^{144}$ While useful for the documentation of all relevant bleeding symptoms in the prospective GAPP study, the ISTH-BAT score was not predictive for platelet function deficiencies, based on lumi-aggregometry measurements. ${ }^{145}$ It should be noted though, that a laboratory defect not always needs to be accompanied by a bleeding phenotype. Since the ISTH-BAT documents on recurrent and mild haemorrhages, which are characteristic for platelet function disorders, further refinement of this BAT may aid in the diagnosis of mild platelet defects.

For inherited platelet disorders bleeding symptoms are usually experienced lifelong, so that retrospective evaluation of the bleeding episodes is of particular importance. On the other hand, sudden (acquired) bleeding due to the use of antiplatelet medication requires description of the acute symptoms. ${ }^{144}$ Detailed registration of bleeding events by physicians is important not only for prediction, but also for safety reasons. Bleeding events related to the use of antiplatelet therapy, whether or not in combination with invasive procedures, are systematically reported, although this occurs not always according to the same bleeding definitions. Records are made of events during clinical trials and after the prescription of approved drugs (particularly Aspirin and $\mathrm{P}_{2} \mathrm{Y}_{12}$ inhibitors). Informative BATs to evaluate such acquired bleeding tendencies have not yet been published. At present, listed are only clinically relevant bleedings, requiring transfusion of red blood cells and/or serious adverse events (e.g. intracranial haemorrhages). In an attempt to accomplish uniformity in bleeding definitions for cardiovascular clinical trials, a consensus classification was published allowing safety comparisons between studies, which deserves proper attention. ${ }^{146}$

\subsection{Laboratory testing of platelet dysfunction}

Particularly in the last years, efforts have been made to standardise laboratory methods for platelet function testing. Quantitative platelet disorders can be detected by measuring platelet count and mean platelet volume in blood samples. Platelet aggregation in platelet-rich plasma in response to a panel of agonists, determined by light transmission aggregometry (LTA), is the current golden standard for detecting 
functional abnormalities. ${ }^{1}$ Since LTA is relatively insensitive in identifying defects in $\delta$-granule secretion, platelet release of ATP needs to be determined additionally, e.g. by lumi-aggregometry. Recommendations have recently been published for the use of standardised LTA tests for UK laboratories ${ }^{147}$ and the ISTH. ${ }^{148}$ A streamlined, validated panel of platelet agonists (ADP, adrenaline, arachidonic acid, collagen, TRAP, ristocetin) has been developed for diagnosing heritable platelet function disorders by testing with LTA and ATP secretion. ${ }^{20}$

Replacing the early method of whole blood impedance aggregometry, the Multiplate and VerifyNow devices are increasingly used for determining platelet aggregation in whole blood, but still require more clinical validation. The PFA-100 is particularly used for testing shear-dependent platelet aggregation in response to collagen/ADP or collagen/adrenaline. The test is capable of detecting severe platelet function defects (Bernard-Soulier, Glanzmann's thrombasthenia) and most types of von Willebrand disease. ${ }^{149}$ However, its sensitivity for less severe defects, like $\delta$-granule storage pool deficiencies, is low. ${ }^{150}$

Flow cytometry is frequently used for specific platelet function testing or for conditions where platelet counts are too low for LTA. ${ }^{151}$ Flow cytometry allows the quantification of platelet receptors, the detection of platelet surface activation markers, and of platelet procoagulant activity. Hence, agonist-induced integrin activation, secretion of $\alpha$ - and $\delta$-granules, and phosphatidylserine exposure can easily be determined. This technique has already been used as a read-out to determine the genetic determinants of platelet signalling pathways. ${ }^{98}$ Flow cytometric tests for assessing (agonist-induced) platelet function in whole blood are in the experimental phase. 152,153

Since platelet activation and coagulation are interdependent processes, ${ }^{3}$ platelet function - in particular procoagulant activity - can also be tested in plateletrich plasma by way of thrombin generation assays., ${ }^{454}$ Recommendations for standardised measurement of platelet-dependent thrombin generation have recently been published by the ISTH. ${ }^{155}$ Developments of a CAT-based assay in whole blood can improve clinical applicability of this type of tests. ${ }^{156}$ However, their predictive value for platelet-related bleeding still needs to be established. Flow chambers have been used to measure thrombus formation in combination with coagulation (fibrin) at defined shear stress conditions. ${ }^{157}$ Efforts are undertaken for better standardisation of these flow-based assays and adaption to smaller volumes of blood. ${ }^{158,159}$

\subsection{Laboratory testing of platelet inhibition}

For optimal patient care it is considered to be important to assess the effectiveness of antiplatelet therapy. This is predominantly an issue in cases of dual antiplatelet therapy with Aspirin and $\mathrm{P}_{2} \mathrm{Y}_{12}$ receptor blockers, as is the standard therapy for patients with acute coronary syndrome or undergoing percutaneous coronary intervention. Due to heterogeneity in individual responses to especially Clopidogrel treatment, inhibition 
of platelet function is suboptimal in a considerable part of the treated patients. This so-called high on-treatment platelet reactivity (HPR) associates with an increased risk of secondary atherothrombotic events. ${ }^{44,160}$ In this context, also the reported variability in responsiveness to Aspirin requires attention. ${ }^{161}$ Platelet function testing in relation to bleeding is also important in patients using the new, potent $\mathrm{P}_{2} \mathrm{Y}_{12}$ blockers, Prasugrel and Ticagrelor, where low on-treatment platelet reactivity (LPR) is a matter of concern. ${ }^{162}$ Together, this would advocate for monitoring platelet reactivity under treatment and concomitant individualised dosing of $\mathrm{P}_{2} \mathrm{Y}_{12}$ blockers.

A platelet function-tailored approach with Clopidogrel may be most effective for patients with a high risk of stent thrombosis. ${ }^{161}$ Further studies, especially with the new $\mathrm{P}_{2} \mathrm{Y}_{12}$ blockers, are required to support the rationale for personalised treatment. ${ }^{160}$ First efforts have been made for defining a therapeutic window for on-treatment platelet reactivity upon intake of $\mathrm{P}_{2} \mathrm{Y}_{12}$ blockers to minimalise both ischemic and bleeding events. ${ }^{162}$ Several platelet function assays are currently available for testing the efficacy and safety of antiplatelet therapy. Although LTA is still the golden standard, currently available point-of-care tests are more convenient for use in daily clinical practice. Of these, the VerifyNow and Multiplate tests may reflect most accurately the variable responsiveness to Aspirin and $P_{2} Y_{12}$ blockers, while the predictive value of the PFA-100 test for $\mathrm{P}_{2} \mathrm{Y}_{12}$ inhibition is limited. ${ }^{163}$

\section{Concluding remarks}

Recent research on platelet responses in whole blood flow studies and experimental thrombosis studies has greatly contributed to our insight into main platelet receptors in thrombosis and haemostasis. In man, haemorrhagic complications can be caused by defective platelet receptors or by therapeutic inhibition of these receptors. It would be important to register these bleeding events in similar ways by using uniform assessment tools and function tests.

\section{Practice points}

- Study of thrombus formation, in whole blood flow studies and experimental murine thrombosis studies, is of relevance to understand the platelet responses in thrombosis and haemostasis in man.

- Main platelet receptors with established or potential clinical relevance are those of thrombin, thromboxane $A_{2}$, ADP, ATP, prostaglandins, von Willebrand factor, collagen, CLEC-2 ligand, fibrinogen and laminin.

- Platelet dysfunction, either inherited or acquired due to medication, may lead to bleeding, but bleeding symptoms are assessed in different ways. 


\section{Research agenda}

- Establishment of the therapeutic window for (potential) antiplatelet agents for optimal efficacy in preventing thrombosis with minimal bleeding.

- Development of bleeding assessment tools for identifying platelet function disorders or low on-treatment platelet reactivity; comparison of these tools to outcome of platelet function tests.

\section{Conflicts of interest}

The authors have obtained research grants from AstraZeneca.

\section{Acknowledgements}

We apologise to those authors who provided important contributions to this broad research field, but whose work could not be cited due to space restrictions. This work was supported by grants from the Dutch Landsteiner Foundation for Blood Transfusion Research (1006) and the Centre for Translational Molecular Medicine (INCOAG).

\section{References}

1. Hayward CP. Diagnostic evaluation of platelet function disorders. Blood Rev. 2011;25:169-173.

2. Mackman N. Triggers, targets and treatments for thrombosis. Nature. 2008;451:914-918.

3. Heemskerk JW, Mattheij NJ, Cosemans JM. Platelet-based coagulation: different populations, different functions. J Thromb Haemost. 2013;11:2-16.

4. Hemker HC, Al Dieri R, Beguin S. Thrombin generation assays: accruing clinical relevance. Curr Opin Hematol. 2004;11:170-175.

5. Burkhart JM, Vaudel M, Gambaryan S, Radau S, Walter U, Martens L, Geiger J, Sickmann A, Zahedi RP. The first comprehensive and quantitative analysis of human platelet protein composition allows the comparative analysis of structural and functional pathways. Blood. 2012;120:e73-82.

6. Michelson AD. Platelets. Amsterdam: Elsevier. 2007.

7. Coughlin SR. Protease-activated receptors in hemostasis, thrombosis and vascular biology. J Thromb Haemost. 2005;3:1800-1814.

8. Kim S, Foster C, Lecchi A, Quinton TM, Prosser DM, Jin J, Cattaneo M, Kunapuli SP. Proteaseactivated receptors 1 and 4 do not stimulate Gi signaling pathways in the absence of secreted ADP and cause human platelet aggregation independently of Gi signaling. Blood. 2002;99:3629-3636.

9. Nurden A, Nurden P. Advances in our understanding of the molecular basis of disorders of platelet function. J Thromb Haemost. 2011;9 Suppl 1:76-91.

10. Weiss EJ, Hamilton JR, Lease KE, Coughlin SR. Protection against thrombosis in mice lacking PAR3. Blood. 2002;100:3240-3244.

11. Hamilton JR, Cornelissen I, Coughlin SR. Impaired hemostasis and protection against thrombosis in protease-activated receptor 4-deficient mice is due to lack of thrombin signaling in platelets. $J$ Thromb Haemost. 2004;2:1429-1435.

12. Vandendries ER, Hamilton JR, Coughlin SR, Furie B, Furie BC. Par4 is required for platelet thrombus propagation but not fibrin generation in a mouse model of thrombosis. Proc Natl Acad Sci U S A. 
2007;104:288-292.

13. Tricoci P, Huang Z, Held C, Moliterno DJ, Armstrong PW, Van de Werf F, White HD, Aylward PE, Wallentin L, Chen E, Lokhnygina Y, Pei J, Leonardi S, Rorick TL, Kilian AM, Jennings LH, Ambrosio G, Bode C, Cequier A, Cornel JH, Diaz R, Erkan A, Huber K, Hudson MP, Jiang L, Jukema JW, Lewis BS, Lincoff AM, Montalescot G, Nicolau JC, Ogawa H, Pfisterer M, Prieto JC, Ruzyllo W, Sinnaeve PR, Storey RF, Valgimigli M, Whellan DJ, Widimsky P, Strony J, Harrington RA, Mahaffey KW. Thrombinreceptor antagonist vorapaxar in acute coronary syndromes. N Engl J Med. 2012;366:20-33.

14. Morrow DA, Braunwald E, Bonaca MP, Ameriso SF, Dalby AJ, Fish MP, Fox KA, Lipka LJ, Liu X, Nicolau JC, Ophuis AJ, Paolasso E, Scirica BM, Spinar J, Theroux P, Wiviott SD, Strony J, Murphy SA. Vorapaxar in the secondary prevention of atherothrombotic events. N Engl J Med. 2012;366:14041413.

15. Scirica BM, Bonaca MP, Braunwald E, De Ferrari GM, Isaza D, Lewis BS, Mehrhof F, Merlini PA, Murphy SA, Sabatine MS, Tendera M, Van de WerfF, Wilcox R, Morrow DA. Vorapaxar for secondary prevention of thrombotic events for patients with previous myocardial infarction: a prespecified subgroup analysis of the TRA 2P-TIMI 50 trial. Lancet. 2012;380:1317-1324.

16. O’Donoghue ML, Bhatt DL, Wiviott SD, Goodman SG, Fitzgerald DJ, Angiolillo DJ, Goto S, Montalescot G, Zeymer U, Aylward PE, Guetta V, Dudek D, Ziecina R, Contant CF, Flather MD. Safety and tolerability of atopaxar in the treatment of patients with acute coronary syndromes: the lessons from antagonizing the cellular effects of Thrombin-Acute Coronary Syndromes Trial. Circulation. 2011;123:1843-1853.

17. Van de Werf F. Inhibitors of the platelet thrombin receptor: will they live up to their promises? Circulation. 2011;123:1833-1835.

18. Li Z, Zhang G, Le Breton GC, Gao X, Malik AB, Du X. Two waves of platelet secretion induced by thromboxane $A_{2}$ receptor and a critical role for phosphoinositide 3-kinases. $J$ Biol Chem. 2003;278:30725-30731.

19. Offermanns S. Activation of platelet function through $G$ protein-coupled receptors. Circ Res. 2006;99:1293-1304.

20. Dawood BB, Lowe GC, Lordkipanidze M, Bem D, Daly ME, Makris M, Mumford A, Wilde JT, Watson SP. Evaluation of participants with suspected heritable platelet function disorders including recommendation and validation of a streamlined agonist panel. Blood. 2012;120:5041-5049.

21. Thomas DW, Mannon RB, Mannon PJ, Latour A, Oliver JA, Hoffman M, Smithies O, Koller BH, Coffman TM. Coagulation defects and altered hemodynamic responses in mice lacking receptors for thromboxane $A_{2}$. J Clin Invest. 1998;102:1994-2001.

22. Cairns JA, Gent M, Singer J, Finnie KJ, Froggatt GM, Holder DA, Jablonsky G, Kostuk WJ, Melendez LJ, Myers MG, Sackett DL, Sealey BJ, Tanser PH. Aspirin, sulfinpyrazone, or both in unstable angina. Results of a Canadian multicenter trial. N Engl J Med. 1985;313:1369-1375.

23. Lewis HD, Jr., Davis JW, Archibald DG, Steinke WE, Smitherman TC, Doherty JE, 3rd, Schnaper HW, LeWinter MM, Linares E, Pouget JM, Sabharwal SC, Chesler E, DeMots H. Protective effects of aspirin against acute myocardial infarction and death in men with unstable angina. Results of a Veterans Administration Cooperative Study. N Engl J Med. 1983;309:396-403.

24. Awtry EH, Loscalzo J. Aspirin. Circulation. 2000;101:1206-1218.

25. Floyd CN, Ferro A. Mechanisms of aspirin resistance. Pharmacol Ther. 2014;141:69-78.

26. Olsen AM, Gislason GH, Fosbol EL. Time-perspective in cardiovascular risk of NSAID use after firsttime myocardial infarction. Curr Opin Cardiol. 2013;28:683-688.

27. Fiessinger JN, Bounameaux H, Cairols MA, Clement DL, Coccheri S, Fletcher JP, Hoffmann U, Turpie AG. Thromboxane Antagonism with terutroban in Peripheral Arterial Disease: the TAIPAD study. J Thromb Haemost. 2010;8:2369-2376.

28. Lesault PF, Boyer L, Pelle G, Covali-Noroc A, Rideau D, Akakpo S, Teiger E, Dubois-Rande JL, Adnot S. Daily administration of the TP receptor antagonist terutroban improved endothelial function in 
high-cardiovascular-risk patients with atherosclerosis. Br J Clin Pharmacol. 2011;71:844-851.

29. Gachet C. P2 receptors, platelet function and pharmacological implications. Thromb Haemost. 2008;99:466-472.

30. Leon C, Hechler B, Freund M, Eckly A, Vial C, Ohlmann P, Dierich A, LeMeur M, Cazenave JP, Gachet $C$. Defective platelet aggregation and increased resistance to thrombosis in purinergic $\mathrm{P}_{2} \mathrm{Y}_{1}$ receptor-null mice. J Clin Invest. 1999;104:1731-1737.

31. Cattaneo $\mathrm{M}$. The platelet $\mathrm{P}_{2} \mathrm{Y}_{12}$ receptor for adenosine diphosphate: congenital and drug-induced defects. Blood. 2011;117:2102-2112.

32. Schoenwaelder SM, Ono A, Sturgeon S, Chan SM, Mangin P, Maxwell MJ, Turnbull S, Mulchandani M, Anderson K, Kauffenstein G, Rewcastle GW, Kendall J, Gachet C, Salem HH, Jackson SP. Identification of a unique co-operative phosphoinositide 3-kinase signaling mechanism regulating integrin $\alpha_{11 b} \beta_{3}$ adhesive function in platelets. J Biol Chem. 2007;282:28648-28658.

33. Garcia A, Kim S, Bhavaraju K, Schoenwaelder SM, Kunapuli SP. Role of phosphoinositide 3-kinase $\beta$ in platelet aggregation and thromboxane $A_{2}$ generation mediated by $G i$ signalling pathways.

Biochem J. 2010;429:369-377.

34. Cosemans JM, Munnix IC, Wetzker R, Heller R, Jackson SP, Heemskerk JW. Continuous signaling via $\mathrm{PI} 3 \mathrm{~K}$ isoforms $\beta$ and $\gamma$ is required for platelet $A D P$ receptor function in dynamic thrombus stabilization. Blood. 2006;108:3045-3052.

35. Fabre JE, Nguyen M, Latour A, Keifer JA, Audoly LP, Coffman TM, Koller BH. Decreased platelet aggregation, increased bleeding time and resistance to thromboembolism in $\mathrm{P}_{2} \mathrm{Y}_{1}$-deficient mice. Nat Med. 1999;5:1199-1202.

36. Hechler B, Zhang Y, Eckly A, Cazenave JP, Gachet C, Ravid K. Lineage-specific overexpression of the $\mathrm{P}_{2} \mathrm{Y}_{1}$ receptor induces platelet hyper-reactivity in transgenic mice. J Thromb Haemost. 2003;1:155163.

37. Hollopeter G, Jantzen HM, Vincent D, Li G, England L, Ramakrishnan V, Yang RB, Nurden P, Nurden A, Julius D, Conley PB. Identification of the platelet ADP receptor targeted by antithrombotic drugs. Nature. 2001;409:202-207.

38. Watson S, Daly M, Dawood B, Gissen P, Makris M, Mundell S, Wilde J, Mumford A. Phenotypic approaches to gene mapping in platelet function disorders - identification of new variant of $\mathrm{P}_{2} \mathrm{Y}_{12}$, $\mathrm{TxA}_{2}$ and GPVI receptors. Hamostaseologie. 2010;30:29-38.

39. Andre P, Delaney SM, LaRocca T, Vincent D, DeGuzman F, Jurek M, Koller B, Phillips DR, Conley PB. $\mathrm{P}_{2} \mathrm{Y}_{12}$ regulates platelet adhesion/activation, thrombus growth, and thrombus stability in injured arteries. J Clin Invest. 2003;112:398-406.

40. Zhang Y, Ye J, Hu L, Zhang S, Zhang SH, Li Y, Kunapuli SP, Ding Z. Increased platelet activation and thrombosis in transgenic mice expressing constitutively active $\mathrm{P}_{2} \mathrm{Y}_{12}$. J Thromb Haemost. 2012;10:2149-2157.

41. Snoep JD, Hovens MM, Eikenboom JC, Van der Bom JG, Jukema JW, Huisman MV. Clopidogrel nonresponsiveness in patients undergoing percutaneous coronary intervention with stenting: a systematic review and meta-analysis. Am Heart J. 2007;154:221-231.

42. Giorgi MA, Cohen Arazi H, Gonzalez CD, Di Girolamo G. Beyond efficacy: pharmacokinetic differences between clopidogrel, prasugrel and ticagrelor. Expert Opin Pharmacother. 2011;12:1285-1295.

43. Roberts JD, Wells GA, Le May MR, Labinaz M, Glover C, Froeschl M, Dick A, Marquis JF, O’Brien E, Goncalves S, Druce I, Stewart A, Gollob MH, So DY. Point-of-care genetic testing for personalisation of antiplatelet treatment (RAPID GENE): a prospective, randomised, proof-of-concept trial. Lancet. 2012;379:1705-1711.

44. Wallentin L, Varenhorst C, James S, Erlinge D, Braun OO, Jakubowski JA, Sugidachi A, Winters KJ, Siegbahn A. Prasugrel achieves greater and faster $\mathrm{P}_{2} \mathrm{Y}_{12}$ receptor-mediated platelet inhibition than clopidogrel due to more efficient generation of its active metabolite in aspirin-treated patients with coronary artery disease. Eur Heart J. 2008;29:21-30. 
45. Pandit A, Aryal MR, Aryal Pandit A, Jalota L, Hakim FA, Mookadam F, Lee HR, Tleyjeh IM. Cangrelor versus clopidogrel in percutaneous coronary intervention: a systematic review and meta-analysis. Eurolntervention. 2014;9:1350-1358.

46. Fung $\mathrm{CY}$, Cendana C, Farndale RW, Mahaut-Smith MP. Primary and secondary agonists can use $\mathrm{P}_{2} \mathrm{X}_{1}$ receptors as a major pathway to increase intracellular $\mathrm{Ca}^{2+}$ in the human platelet. $J$ Thromb Haemost. 2007;5:910-917.

47. Oury C, Toth-Zsamboki E, Van Geet C, Thys C, Wei L, Nilius B, Vermylen J, Hoylaerts MF. A natural dominant negative $\mathrm{P}_{2} \mathrm{X}_{1}$ receptor due to deletion of a single amino acid residue. J Biol Chem. 2000;275:22611-22614.

48. Hechler B, Lenain N, Marchese P, Vial C, Heim V, Freund M, Cazenave JP, Cattaneo M, Ruggeri ZM, Evans $\mathrm{R}$, Gachet $\mathrm{C}$. A role of the fast ATP-gated $\mathrm{P}_{2} \mathrm{X}_{1}$ cation channel in thrombosis of small arteries in vivo. J Exp Med. 2003;198:661-667.

49. Mulryan K, Gitterman DP, Lewis CJ, Vial C, Leckie BJ, Cobb AL, Brown JE, Conley EC, Buell G, Pritchard CA, Evans RJ. Reduced vas deferens contraction and male infertility in mice lacking $\mathrm{P}_{2} \mathrm{X}_{1}$ receptors. Nature. 2000;403:86-89.

50. Mustard JF, Kinlough-Rathbone RL, Packham MA. Prostaglandins and platelets. Annu Rev Med. 1980;31:89-96.

51. Gegenbauer K, Elia G, Blanco-Fernandez A, Smolenski A. Regulator of G-protein signaling 18 integrates activating and inhibitory signaling in platelets. Blood. 2012;119:3799-3807.

52. Subramanian H, Zahedi RP, Sickmann A, Walter U, Gambaryan S. Phosphorylation of CalDAG-GEFI by protein kinase A prevents Rap1b activation. J Thromb Haemost. 2013;11:1574-1582.

53. Murata T, Ushikubi F, Matsuoka T, Hirata M, Yamasaki A, Sugimoto Y, Ichikawa A, Aze Y, Tanaka T, Yoshida N, Ueno A, Oh-ishi S, Narumiya S. Altered pain perception and inflammatory response in mice lacking prostacyclin receptor. Nature. 1997;388:678-682.

54. Stitham J, Arehart E, Elderon L, Gleim SR, Douville K, Kasza Z, Fetalvero K, MacKenzie T, Robb J, Martin KA, Hwa J. Comprehensive biochemical analysis of rare prostacyclin receptor variants: study of association of signaling with coronary artery obstruction. J Biol Chem. 2011;286:7060-7069.

55. Petrucci G, De Cristofaro R, Rutella S, Ranelletti FO, Pocaterra D, Lancellotti S, Habib A, Patrono C, Rocca B. Prostaglandin $E_{2}$ differentially modulates human platelet function through the prostanoid EP2 and EP3 receptors. J Pharmacol Exp Ther. 2011;336:391-402.

56. lyu D, Glenn JR, White AE, Johnson AJ, Fox SC, Heptinstall S. The role of prostanoid receptors in mediating the effects of $P G E_{2}$ on human platelet function. Platelets. 2010;21:329-342.

57. Philipose S, Konya V, Sreckovic I, Marsche G, Lippe IT, Peskar BA, Heinemann A, Schuligoi R. The prostaglandin $E_{2}$ receptor $E_{4}$ is expressed by human platelets and potently inhibits platelet aggregation and thrombus formation. Arterioscler Thromb Vasc Biol. 2010;30:2416-2423.

58. Heptinstall S, Espinosa DI, Manolopoulos P, Glenn JR, White AE, Johnson A, Dovlatova N, Fox SC, May JA, Hermann D, Magnusson O, Stefansson K, Hartman D, Gurney M. DG-041 inhibits the EP3 prostanoid receptor - a new target for inhibition of platelet function in atherothrombotic disease. Platelets. 2008;19:605-613.

59. Singh J, Zeller W, Zhou N, Hategen G, Mishra R, Polozov A, Yu P, Onua E, Zhang J, Zembower D, Kiselyov A, Ramirez JL, Sigthorsson G, Bjornsson JM, Thorsteinsdottir M, Andresson T, Bjarnadottir M, Magnusson O, Fabre JE, Stefansson K, Gurney ME. Antagonists of the EP3 receptor for prostaglandin $E_{2}$ are novel antiplatelet agents that do not prolong bleeding. ACS Chem Biol. 2009;4:115-126.

60. Fox SC, May JA, Johnson A, Hermann D, Strieter D, Hartman D, Heptinstall S. Effects on platelet function of an $\mathrm{EP}_{3}$ receptor antagonist used alone and in combination with a $\mathrm{P}_{2} \mathrm{Y}_{12}$ antagonist both in-vitro and ex-vivo in human volunteers. Platelets. 2013;24:392-400.

61. De Ceunynck K, De Meyer SF, Vanhoorelbeke K. Unwinding the von Willebrand factor strings puzzle. Blood. 2013;121:270-277. 
62. Yago T, Lou J, Wu T, Yang J, Miner JJ, Coburn L, Lopez JA, Cruz MA, Dong JF, McIntire LV, McEver $\mathrm{RP}$, Zhu C. Platelet glycoprotein Iba forms catch bonds with human WT vWF but not with type 2B von Willebrand disease vWF. J Clin Invest. 2008;118:3195-3207.

63. Auger JM, Kuijpers MJ, Senis YA, Watson SP, Heemskerk JW. Adhesion of human and mouse platelets to collagen under shear: a unifying model. FASEB J. 2005;19:825-827.

64. Ruggeri ZM. The role of von Willebrand factor in thrombus formation. Thromb Res. 2007;120 Suppl 1:S5-9.

65. Nesbitt WS, Westein E, Tovar-Lopez FJ, Tolouei E, Mitchell A, Fu J, Carberry J, Fouras A, Jackson SP. A shear gradient-dependent platelet aggregation mechanism drives thrombus formation. Nat Med. 2009;15:665-673.

66. Westein E, Van der Meer AD, Kuijpers MJ, Frimat JP, Van den Berg A, Heemskerk JW. Atherosclerotic geometries exacerbate pathological thrombus formation poststenosis in a von Willebrand factordependent manner. Proc Natl Acad Sci U S A. 2013;110:1357-1362.

67. Kasirer-Friede A, Cozzi MR, Mazzucato M, De Marco L, Ruggeri ZM, Shattil SJ. Signaling through GP Ib-IX-V activates $\alpha_{11 b} \beta_{3}$ independently of other receptors. Blood. 2004;103:3403-3411.

68. Du X. Signaling and regulation of the platelet glycoprotein Ib-IX-V complex. Curr Opin Hematol. 2007;14:262-269.

69. Canobbio I, Bertoni A, Lova P, Paganini S, Hirsch E, Sinigaglia F, Balduini C, Torti M. Platelet activation by von Willebrand factor requires coordinated signaling through thromboxane $A_{2}$ and FcyllA receptor. J Biol Chem. 2001;276:26022-26029.

70. De Candia E, Hall SW, Rutella S, Landolfi R, Andrews RK, De Cristofaro R. Binding of thrombin to glycoprotein Ib accelerates the hydrolysis of PAR-1 on intact platelets. J Biol Chem. 2001;276:46924698.

71. Cosemans JM, Schols SE, Stefanini L, De Witt S, Feijge MA, Hamulyák K, Deckmyn H, Bergmeier W, Heemskerk JW. Key role of glycoprotein Ib/V/IX and von Willebrand factor in platelet activationdependent fibrin formation at low shear flow. Blood. 2011;117:651-660.

72. Bergmeier W, Chauhan AK, Wagner DD. Glycoprotein Iba and von Willebrand factor in primary platelet adhesion and thrombus formation: lessons from mutant mice. Thromb Haemost. 2008;99:264-270.

73. Lenting PJ, Casari C, Christophe OD, Denis CV. von Willebrand factor: the old, the new and the unknown. J Thromb Haemost. 2012;10:2428-2437.

74. Kato K, Martinez C, Russell S, Nurden P, Nurden A, Fiering S, Ware J. Genetic deletion of mouse platelet glycoprotein $\mathrm{lb} \beta$ produces a Bernard-Soulier phenotype with increased a-granule size. Blood. 2004;104:2339-2344.

75. Ware J, Russell S, Ruggeri ZM. Generation and rescue of a murine model of platelet dysfunction: the Bernard-Soulier syndrome. Proc Natl Acad Sci U S A. 2000;97:2803-2808.

76. Ramakrishnan V, DeGuzman F, Bao M, Hall SW, Leung LL, Phillips DR. A thrombin receptor function for platelet glycoprotein Ib-IX unmasked by cleavage of glycoprotein V. Proc Natl Acad Sci U S A. 2001;98:1823-1828.

77. Fontayne A, Meiring M, Lamprecht S, Roodt J, Demarsin E, Barbeaux P, Deckmyn H. The humanized anti-glycoprotein Ib monoclonal antibody h6B4-Fab is a potent and safe antithrombotic in a high shear arterial thrombosis model in baboons. Thromb Haemost. 2008;100:670-677.

78. Wu D, Meiring M, Kotze HF, Deckmyn H, Cauwenberghs N. Inhibition of platelet glycoprotein Ib, glycoprotein IIb/IIla, or both by monoclonal antibodies prevents arterial thrombosis in baboons. Arterioscler Thromb Vasc Biol. 2002;22:323-328.

79. Lillicrap D. Von Willebrand disease - phenotype versus genotype: deficiency versus disease. Thromb Res. 2007;120 Suppl 1:S11-16.

80. Nieswandt B, Watson SP. Platelet-collagen interaction: is GPVI the central receptor? Blood. 2003;102:449-461. 
81. Jung SM, Moroi M, Soejima K, Nakagaki T, Miura Y, Berndt MC, Gardiner EE, Howes JM, Pugh $\mathrm{N}$, Bihan D, Watson SP, Farndale RW. Constitutive dimerization of glycoprotein VI (GPVI) in resting platelets is essential for binding to collagen and activation in flowing blood. J Biol Chem. 2012;287:30000-30013.

82. Munnix IC, Kuijpers MJ, Auger J, Thomassen CM, Panizzi P, Van Zandvoort MA, Rosing J, Bock PE, Watson SP, Heemskerk JW. Segregation of platelet aggregatory and procoagulant microdomains in thrombus formation: regulation by transient integrin activation. Arterioscler Thromb Vasc Biol. 2007;27:2484-2490.

83. Stegner D, Nieswandt B. Platelet receptor signaling in thrombus formation. J Mol Med (Berl). 2011;89:109-121.

84. Watson SP, Auger JM, McCarty OJ, Pearce AC. GPVI and integrin $\alpha_{11 \mathrm{~b}} \beta_{3}$ signaling in platelets. J. Thromb Haemost. 2005;3:1752-1762.

85. Senis YA. Protein-tyrosine phosphatases: a new frontier in platelet signal transduction. $J$ Thromb Haemost. 2013;11:1800-1813.

86. Gilio K, Munnix IC, Mangin P, Cosemans JM, Feijge MA, Van der Meijden PE, Olieslagers S, Chrzanowska-Wodnicka MB, Lillian R, Schoenwaelder S, Koyasu S, Sage SO, Jackson SP, Heemskerk $J W$. Non-redundant roles of phosphoinositide 3-kinase isoforms $\alpha$ and $\beta$ in glycoprotein $\mathrm{Vl}$-induced platelet signaling and thrombus formation. J Biol Chem. 2009;284:33750-33762.

87. Tomlinson MG, Calaminus SD, Berlanga O, Auger JM, Bori-Sanz T, Meyaard L, Watson SP. Collagen promotes sustained glycoprotein VI signaling in platelets and cell lines. J Thromb Haemost. 2007;5:2274-2283.

88. Heemskerk JW, Harper MT, Cosemans JM, Poole AW. Unravelling the different functions of protein kinase $C$ isoforms in platelets. FEBS Lett. 2011;585:1711-1716.

89. Stefanini L, Roden RC, Bergmeier W. CalDAG-GEFI is at the nexus of calcium-dependent platelet activation. Blood. 2009;114:2506-2514.

90. Düutting S, Bender M, Nieswandt B. Platelet GPVI: a target for antithrombotic therapy?! Trends Pharmacol Sci. 2012;33:583-590.

91. Schulte V, Rabie T, Prostredna M, Aktas B, Gruner S, Nieswandt B. Targeting of the collagen-binding site on glycoprotein VI is not essential for in vivo depletion of the receptor. Blood. 2003;101:39483952.

92. Kuijpers MJ, Gilio K, Reitsma S, Nergiz-Unal R, Prinzen L, Heeneman S, Lutgens E, Van Zandvoort MA, Nieswandt B, Egbrink MG, Heemskerk JW. Complementary roles of platelets and coagulation in thrombus formation on plaques acutely ruptured by targeted ultrasound treatment: a novel intravital model. J Thromb Haemost. 2009;7:152-161.

93. Dubois C, Panicot-Dubois L, Merrill-Skoloff G, Furie B, Furie BC. Glycoprotein VI-dependent and -independent pathways of thrombus formation in vivo. Blood. 2006;107:3902-3906.

94. Hechler B, Nonne C, Eckly A, Magnenat S, Rinckel JY, Denis CV, Freund M, Cazenave JP, Lanza F, Gachet C. Arterial thrombosis: relevance of a model with two levels of severity assessed by histologic, ultrastructural and functional characterization. J Thromb Haemost. 2010;8:173-184.

95. Zahid M, Mangin P, Loyau S, Hechler B, Billiald P, Gachet C, Jandrot-Perrus M. The future of glycoprotein VI as an antithrombotic target. J Thromb Haemost. 2012;10:2418-2427.

96. Matus V, Valenzuela G, Saez CG, Hidalgo P, Lagos M, Aranda E, Panes O, Pereira J, Pillois X, Nurden AT, Mezzano D. An adenine insertion in exon 6 of human GP6 generates a truncated protein associated with a bleeding disorder in four Chilean families. J Thromb Haemost. 2013;11:1751-1759.

97. Johnson AD, Yanek LR, Chen MH, Faraday N, Larson MG, Tofler G, Lin SJ, Kraja AT, Province MA, Yang Q, Becker DM, O’Donnell CJ, Becker LC. Genome-wide meta-analyses identifies seven loci associated with platelet aggregation in response to agonists. Nat Genet. 2010;42:608-613.

98. Jones Cl, Bray S, Garner SF, Stephens J, De Bono B, Angenent WG, Bentley D, Burns P, Coffey A, Deloukas P, Earthrowl M, Farndale RW, Hoylaerts MF, Koch K, Rankin A, Rice CM, Rogers J, 
Samani NJ, Steward M, Walker A, Watkins NA, Akkerman JW, Dudbridge F, Goodall AH, Ouwehand $\mathrm{WH}$. A functional genomics approach reveals novel quantitative trait loci associated with platelet signaling pathways. Blood. 2009;114:1405-1416.

99. Kuijpers MJ, Schulte V, Bergmeier W, Lindhout T, Brakebusch C, Offermanns S, Fassler R, Heemskerk $J W$, Nieswandt B. Complementary roles of glycoprotein VI and $\alpha_{2} \beta_{1}$ integrin in collagen-induced thrombus formation in flowing whole blood ex vivo. FASEB J. 2003;17:685-687.

100. Nieswandt B, Brakebusch C, Bergmeier W, Schulte V, Bouvard D, Mokhtari-Nejad R, Lindhout T, Heemskerk JW, Zirngibl H, Fässler R. Glycoprotein VI but not $\alpha_{2} \beta_{1}$ integrin is essential for platelet interaction with collagen. EMBO J. 2001;20:2120-2130.

101. Munnix IC, Strehl A, Kuijpers MJ, Auger JM, Van der Meijden PE, Van Zandvoort MA, Oude Egbrink MG, Nieswandt B, Heemskerk JW. The glycoprotein VI-phospholipase C ${ }^{2} 2$ signaling pathway controls thrombus formation induced by collagen and tissue factor in vitro and in vivo. Arterioscler Thromb Vasc Biol. 2005;25:2673-2678.

102. Elvers M, Pozgaj R, Pleines I, May F, Kuijpers MJ, Heemskerk JM, Yu P, Nieswandt B. Platelet hyperreactivity and a prothrombotic phenotype in mice with a gain-of-function mutation in phospholipase C $\gamma 2$. J Thromb Haemost. 2010;8:1353-1363.

103. Watson SP, Herbert JM, Pollitt AY. GPVI and CLEC-2 in hemostasis and vascular integrity. J Thromb Haemost. 2010;8:1456-1467.

104. Lecut C, Schoolmeester A, Kuijpers MJ, Broers JL, Van Zandvoort MA, Vanhoorelbeke K, Deckmyn $\mathrm{H}$, Jandrot-Perrus $M$, Heemskerk JW. Principal role of glycoprotein $V I$ in $\alpha_{2} \beta_{1}$ and $\alpha_{11 b} \beta_{3}$ activation during collagen-induced thrombus formation. Arterioscler Thromb Vasc Biol. 2004;24:1727-1733.

105. Siljander PR, Munnix IC, Smethurst PA, Deckmyn H, Lindhout T, Ouwehand WH, Farndale RW, Heemskerk JW. Platelet receptor interplay regulates collagen-induced thrombus formation in flowing human blood. Blood. 2004;103:1333-1341.

106. Matsumoto Y, Takizawa H, Nakama K, Gong X, Yamada Y, Tandon NN, Kambayashi J. Ex vivo evaluation of anti-GPVI antibody in cynomolgus monkeys: dissociation between anti-platelet aggregatory effect and bleeding time. Thromb Haemost. 2006;96:167-175.

107. Massberg S, Konrad I, Bultmann A, Schulz C, Munch G, Peluso M, Lorenz M, Schneider S, Besta F, Muller I, Hu B, Langer H, Kremmer E, Rudelius M, Heinzmann U, Ungerer M, Gawaz M. Soluble glycoprotein VI dimer inhibits platelet adhesion and aggregation to the injured vessel wall in vivo. FASEB J. 2004;18:397-399.

108. Ungerer M, Rosport K, Bultmann A, Piechatzek R, Uhland K, Schlieper P, Gawaz M, Munch G. Novel antiplatelet drug revacept (Dimeric Glycoprotein VI-Fc) specifically and efficiently inhibited collagen-induced platelet aggregation without affecting general hemostasis in humans. Circulation. 2011;123:1891-1899.

109. Navarro-Nunez L, Langan SA, Nash GB, Watson SP. The physiological and pathophysiological roles of platelet CLEC-2. Thromb Haemost. 2013;109:991-998.

110. Suzuki-Inoue K, Inoue O, Ozaki Y. Novel platelet activation receptor CLEC-2: from discovery to prospects. J Thromb Haemost. 2011;9 Suppl 1:44-55.

111. Parguina AF, Alonso J, Rosa I, Velez P, Gonzalez-Lopez MJ, Guitian E, Eble JA, Loza MI, Garcia A. A detailed proteomic analysis of rhodocytin-activated platelets reveals novel clues on the CLEC-2 signalosome: implications for CLEC-2 signaling regulation. Blood. 2012;120:e117-126.

112. May F, Hagedorn I, Pleines I, Bender M, Vogtle T, Eble J, Elvers M, Nieswandt B. CLEC-2 is an essential platelet-activating receptor in hemostasis and thrombosis. Blood. 2009;114:3464-3472.

113. Bender M, May F, Lorenz V, Thielmann I, Hagedorn I, Finney BA, Vogtle T, Remer K, Braun A, BosI $M$, Watson SP, Nieswandt B. Combined in vivo depletion of glycoprotein VI and C-type lectinlike receptor 2 severely compromises hemostasis and abrogates arterial thrombosis in mice. Arterioscler Thromb Vasc Biol. 2013;33:926-934.

114. Moser M, Nieswandt B, Ussar S, Pozgajova M, Fassler R. Kindlin-3 is essential for integrin activation 
and platelet aggregation. Nat Med. 2008;14:325-330.

115. Nieswandt B, Varga-Szabo D, Elvers M. Integrins in platelet activation. J Thromb Haemost. 2009;7 Suppl 1:206-209.

116. Petrich BG, Marchese P, Ruggeri ZM, Spiess S, Weichert RA, Ye F, Tiedt R, Skoda RC, Monkley SJ, Critchley DR, Ginsberg MH. Talin is required for integrin-mediated platelet function in hemostasis and thrombosis. J Exp Med. 2007;204:3103-3111.

117. Shattil SJ. Signaling through platelet integrin $\alpha_{11 b} \beta_{3}$ : inside-out, outside-in, and sideways. Thromb Haemost. 1999;82:318-325.

118. Van der Meijden PE, Feijge MA, Swieringa F, Gilio K, Nergiz-Unal R, Hamulyák K, Heemskerk JW. Key role of integrin $\alpha_{11 b} \beta_{3}$ signaling to Syk kinase in tissue factor-induced thrombin generation. Cell Mol Life Sci. 2012;69:3481-3492.

119. Nurden AT, Fiore M, Nurden P, Pillois X. Glanzmann thrombasthenia: a review of ITGA2B and ITGB3 defects with emphasis on variants, phenotypic variability, and mouse models. Blood. 2011;118:59966005.

120. Hodivala-Dilke KM, McHugh KP, Tsakiris DA, Rayburn H, Crowley D, Ullman-Cullere M, Ross FP, Coller BS, Teitelbaum S, Hynes RO. $\beta_{3}$-integrin-deficient mice are a model for Glanzmann thrombasthenia showing placental defects and reduced survival. J Clin Invest. 1999;103:229-238.

121. Tronik-Le Roux D, Roullot V, Poujol C, Kortulewski T, Nurden P, Marguerie G. Thrombasthenic mice generated by replacement of the integrin $\alpha_{11 b}$ gene: demonstration that transcriptional activation of this megakaryocytic locus precedes lineage commitment. Blood. 2000;96:1399-1408.

122. Malinin NL, Zhang L, Choi J, Ciocea A, Razorenova O, Ma YQ, Podrez EA, Tosi M, Lennon DP, Caplan Al, Shurin SB, Plow EF, Byzova TV. A point mutation in KINDLIN3 ablates activation of three integrin subfamilies in humans. Nat Med. 2009;15:313-318.

123. Svensson L, Howarth K, McDowall A, Patzak I, Evans R, Ussar S, Moser M, Metin A, Fried M, Tomlinson I, Hogg N. Leukocyte adhesion deficiency-III is caused by mutations in KINDLIN3 affecting integrin activation. Nat Med. 2009;15:306-312.

124. Leopold JA. Small-molecule glycoprotein IIb/IIla antagonists and bleeding risk in women: too much of a good thing? Circulation. 2006;114:1344-1346.

125. Muniz-Lozano A, Rollini F, Franchi F, Angiolillo DJ. Update on platelet glycoprotein IIb/IIla inhibitors: recommendations for clinical practice. Ther Adv Cardiovasc Dis. 2013;7:197-213.

126. Armstrong PC, Peter K. GPIIb/IIla inhibitors: from bench to bedside and back to bench again. Thromb Haemost. 2012;107:808-814.

127. Van de Vijver E, De Cuyper IM, Gerrits AJ, Verhoeven AJ, Seeger K, Gutierrez L, Van den Berg TK, Kuijpers TW. Defects in Glanzmann thrombasthenia and LAD-III (LAD-1/v) syndrome: the role of integrin $\beta_{1}$ and $\beta_{3}$ in platelet adhesion to collagen. Blood. 2012;119:583-586.

128. Kuijpers J, Pozgajova M, Cosemans JM, Munnix IC, Eckes B, Nieswandt B, Heemskerk JW. Role of murine integrin $\alpha_{2} \beta_{1}$ in thrombus stabilization and embolization: contribution of thromboxane $A_{2}$. Thromb Haemost. 2007;98:1072-1080.

129. Chen J, Diacovo TG, Grenache DG, Santoro SA, Zutter MM. The $\alpha_{2}$ integrin subunit-deficient mouse: a multifaceted phenotype including defects of branching morphogenesis and hemostasis. Am J Pathol. 2002;161:337-344.

130. Gruner S, Prostredna M, Aktas B, Moers A, Schulte V, Krieg T, Offermanns S, Eckes B, Nieswandt B. Anti-glycoprotein VI treatment severely compromises hemostasis in mice with reduced $\alpha_{2} \beta_{1}$ levels or concomitant aspirin therapy. Circulation. 2004;110:2946-2951.

131. Petzold T, Ruppert R, Pandey D, Barocke V, Meyer H, Lorenz M, Zhang L, Siess W, Massberg S, Moser $M . \beta_{1}$ integrin-mediated signals are required for platelet granule secretion and hemostasis in mouse. Blood. 2013;122:2723-2731.

132. Kehrel B, Balleisen L, Kokott R, Mesters R, Stenzinger W, Clemetson KJ, Van de Loo J. Deficiency of intact thrombospondin and membrane glycoprotein la in platelets with defective collagen- 
induced aggregation and spontaneous loss of disorder. Blood. 1988;71:1074-1078.

133. Nieuwenhuis HK, Akkerman JW, Houdijk WP, Sixma JJ. Human blood platelets showing no response to collagen fail to express surface glycoprotein la. Nature. 1985;318:470-472.

134. Schaff M, Tang C, Maurer E, Bourdon C, Receveur N, Eckly A, Hechler B, Arnold C, De Arcangelis A, Nieswandt B, Denis CV, Lefebvre O, Georges-Labouesse E, Gachet C, Lanza F, Mangin PH. Integrin $\alpha_{6} \beta_{1}$ is the main receptor for vascular laminins and plays a role in platelet adhesion, activation, and arterial thrombosis. Circulation. 2013;128:541-552.

135. Versteeg HH, Heemskerk JW, Levi M, Reitsma PH. New fundamentals in hemostasis. Physiol Rev. 2013;93:327-358.

136. Brass LF, Wannemacher KM, Ma P, Stalker TJ. Regulating thrombus growth and stability to achieve an optimal response to injury. J Thromb Haemost. 2011;9 Suppl 1:66-75.

137. Cosemans JM, Angelillo-Scherrer A, Mattheij NJ, Heemskerk JW. The effects of arterial flow on platelet activation, thrombus growth, and stabilization. Cardiovasc Res. 2013;99:342-352.

138. Jackson SP. The growing complexity of platelet aggregation. Blood. 2007;109:5087-5095.

139. Watson SP, Lowe GC, Lordkipanidze M, Morgan NV. Genotyping and phenotyping of platelet function disorders. J Thromb Haemost. 2013;11 Suppl 1:351-363.

140. Deppermann C, Cherpokova D, Nurden P, Schulz JN, Thielmann I, Kraft P, Vogtle T, Kleinschnitz C, Dutting S, Krohne G, Eming SA, Nurden AT, Eckes B, Stoll G, Stegner D, Nieswandt B. Gray platelet syndrome and defective thrombo-inflammation in Nbeal2-deficient mice. $J$ Clin Invest. 2013;123:3331-3342.

141. Nylander S, Kull B, Bjorkman JA, Ulvinge JC, Oakes N, Emanuelsson BM, Andersson M, Skarby $\mathrm{T}$, Inghardt T, Fjellstrom O, Gustafsson D. Human target validation of phosphoinositide 3-kinase (PI3K)B: effects on platelets and insulin sensitivity, using AZD6482 a novel PI3K $\beta$ inhibitor. J Thromb Haemost. 2012;10:2127-2136.

142. Mauer AC, Khazanov NA, Levenkova N, Tian S, Barbour EM, Khalida C, Tobin JN, Coller BS. Impact of sex, age, race, ethnicity and aspirin use on bleeding symptoms in healthy adults. J Thromb Haemost. 2011;9:100-108.

143. Rydz N, James PD. The evolution and value of bleeding assessment tools. J Thromb Haemost. 2012;10:2223-2229.

144. Rodeghiero F, Tosetto A, Abshire T, Arnold DM, Coller B, James P, Neunert C, Lillicrap D. ISTH/SSC bleeding assessment tool: a standardized questionnaire and a proposal for a new bleeding score for inherited bleeding disorders. J Thromb Haemost. 2010;8:2063-2065.

145. Lowe GC, Lordkipanidze M, Watson SP. Utility of the ISTH bleeding assessment tool in predicting platelet defects in participants with suspected inherited platelet function disorders. J Thromb Haemost. 2013;11:1663-1668.

146. Mehran R, Rao SV, Bhatt DL, Gibson CM, Caixeta A, Eikelboom J, Kaul S, Wiviott SD, Menon V, Nikolsky E, Serebruany V, Valgimigli M, Vranckx P, Taggart D, Sabik JF, Cutlip DE, Krucoff MW, Ohman EM, Steg PG, White H. Standardized bleeding definitions for cardiovascular clinical trials: a consensus report from the Bleeding Academic Research Consortium. Circulation. 2011;123:27362747.

147. Harrison P, Mackie I, Mumford A, Briggs C, Liesner R, Winter M, Machin S. Guidelines for the laboratory investigation of heritable disorders of platelet function. Br J Haematol. 2011;155:30-44.

148. Cattaneo M, Cerletti C, Harrison P, Hayward CP, Kenny D, Nugent D, Nurden P, Rao AK, Schmaier AH, Watson SP, Lussana F, Pugliano MT, Michelson AD. Recommendations for the standardization of light transmission aggregometry: a consensus of the working party from the Platelet Physiology Subcommittee of SSC/ISTH. J Thromb Haemost. 2013;11:1183-1189.

149. Hayward CP, Harrison P, Cattaneo M, Ortel TL, Rao AK. Platelet function analyzer (PFA)-10o closure time in the evaluation of platelet disorders and platelet function. J Thromb Haemost. 2006;4:312319. 
150. Sladky JL, Klima J, Grooms L, Kerlin BA, O’Brien SH. The PFA-100 ${ }^{\circledR}$ does not predict delta-granule platelet storage pool deficiencies. Haemophilia. 2012;18:626-629.

151. Goodall AH, Appleby J. Flow-cytometric analysis of platelet-membrane glycoprotein expression and platelet activation. Methods Mol Biol. 2004;272:225-253.

152. Fox SC, May JA, Shah A, Neubert U, Heptinstall S. Measurement of platelet P-selectin for remote testing of platelet function during treatment with clopidogrel and/or aspirin. Platelets. 2009;20:250259.

153. Roest M, Van Holten TC, Fleurke GJ, Remijn JA. Platelet activation test in unprocessed blood (Pac$\mathrm{t}-\mathrm{UB}$ ) to monitor platelet concentrates and whole blood of thrombocytopenic patients. Transfus Med Hemother. 2013;40:117-125.

154. Vanschoonbeek K, Feijge MA, Van Kampen RJ, Kenis H, Hemker HC, Giesen PL, Heemskerk JW. Initiating and potentiating role of platelets in tissue factor-induced thrombin generation in the presence of plasma: subject-dependent variation in thrombogram characteristics. J Thromb Haemost. 2004;2:476-484.

155. Subcommittee on control of anticoagulation of the SSC of the ISTH. Towards a recommendation for the standardization of the measurement of platelet-dependent thrombin generation. J Thromb Haemost. 2011;9:1859-1861.

156. Ninivaggi M, Apitz-Castro R, Dargaud Y, De Laat B, Hemker HC, Lindhout T. Whole-blood thrombin generation monitored with a calibrated automated thrombogram-based assay. Clin Chem. 2012;58:1252-1259.

157. Berny MA, Munnix IC, Auger JM, Schols SE, Cosemans JM, Panizzi P, Bock PE, Watson SP, McCarty OJ, Heemskerk JW. Spatial distribution of factor Xa, thrombin, and fibrin(ogen) on thrombi at venous shear. PLoS One. 2010;5:e10415.

158. Roest M, Reininger A, Zwaginga JJ, King MR, Heemskerk JW. Flow chamber-based assays to measure thrombus formation in vitro: requirements for standardization. J Thromb Haemost. 2011;9:2322-2324.

159. Van Kruchten R, Cosemans JM, Heemskerk JW. Measurement of whole blood thrombus formation using parallel-plate flow chambers - a practical guide. Platelets. 2012;23:229-242.

160. Cattaneo M. New $\mathrm{P}_{2} \mathrm{Y}_{12}$ inhibitors. Circulation. 2010;121:171-179.

161. Janssen PW, Ten Berg JM. Platelet function testing and tailored antiplatelet therapy. J CardiovasC Transl Res. 2013;6:316-328.

162. Tantry US, Bonello L, Aradi D, Price MJ, Jeong YH, Angiolillo DJ, Stone GW, Curzen N, Geisler T, Ten Berg J, Kirtane A, Siller-Matula J, Mahla E, Becker RC, Bhatt DL, Waksman R, Rao SV, Alexopoulos D, Marcucci R, Reny JL, Trenk D, Sibbing D, Gurbel PA. Consensus and update on the definition of on-treatment platelet reactivity to adenosine diphosphate associated with ischemia and bleeding. J Am Coll Cardiol. 2013;62:2261-2273.

163. Tantry US, Gurbel PA. Assessment of oral antithrombotic therapy by platelet function testing. Nat Rev Cardiol. 2011;8:572-579. 



\section{CHAPTER 3}

Multiple changes in novel protein kinase A phosphorylation targets identified in platelets from patient with impaired Gsa signalling

Swieringa F, Solari FA, Feijge MA, Mattheij NJ, Beck F, Stumpel CT, Sickmann A, Van der Meijden PE, Körver-Keularts IM, Zahedi RP, Heemskerk JW

Submitted 


\begin{abstract}
Patients with Albright hereditary osteodystrophy (AHO) are diagnosed with pseudohypoparathyroidism, with as common feature impaired signalling of parathyroid hormone and other hormones, when inherited maternally. The syndrome is accompanied by mutations in the GNAS complex gene locus for Gsa, resulting in impaired cellular signalling via Gsa, adenylate cyclase and CAMP-dependent protein kinase $\mathrm{A}$ (PKA). In platelets from patients with $\mathrm{AHO}$, we observed a partially impaired ability of prostaglandins (prostaglandin $E_{1}$ or iloprost) to inhibit multiple platelet functions in terms of platelet aggregation, secretion and thrombus formation. By using novel and sensitive proteomics technologies, we for the first time determined in platelets from an $\mathrm{AHO}$ patient the changes in the protein abundance and protein phosphorylation pattern that are: (i) linked to defective Gsa activity, (ii) mediated by PKA and, (iii) explanatory for the multiple changes in platelet functions. Whereas the global proteome was in essence unchanged, multiple proteins of the following categories had sites of reduced phosphorylation in the patient: Cytoskeleton actinmyosin, Membrane receptors \& channels, Protein kinases \& phosphatases, Signalling \& adapter proteins, and Small GTPases \& regulators. Taken together, this work demonstrates that quantitative platelet phosphoproteomics can be a powerful tool to detect altered phosphorylation in patients in accordance with the aberrant signalling expected from the diagnosis. We furthermore provide a list of 180 iloproststimulated, mostly PKA-mediated protein phosphorylation events that can be used for the assessment of altered signal transduction of platelets in $\mathrm{AHO}$ and related diseases.
\end{abstract}

\title{
Introduction
}

The G-protein a-subunit, Gsa, is encoded by the imprinted complex gene locus GNAS. ${ }^{1-3}$ When activated through a variety of Gsa-linked transmembrane receptors, it acts as a main stimulating effector of adenylate cyclase (AC), which produces the ubiquitous second messenger CAMP. ${ }^{4}$ Elevation in CAMP triggers several intracellular signalling pathways, in particular mediated by the broad-spectrum serine/threonine CAMP-dependent protein kinase A (PKA). ${ }^{5}$ The classical signalling route of GsaAC-PKA is active in all cells, and regulates multiple physiological processes such as developmental, differentiation and metabolic activities, as well as secretory and cytoskeletal responses. ${ }^{6}$ Despite the abundant presence of PKA however, relatively little is known of its protein substrates in specific cell systems.

In platelets, the Gsa-AC-PKA pathway is one of the most powerful inhibitory pathways of activation processes. Elevation in CAMP - next to CGMP - is a main mechanism to keep platelets quiescent while circulating in the blood and thus, to prevent adhesion, shape change, aggregation and release of autocrine and paracrine stimulating agents. ${ }^{7-9}$ Key triggers of the PKA pathway are the Gsa-coupled IP and EP 
receptors, ${ }^{10}$ which bind endothelial-derived prostaglandin $\mathrm{I}_{2}$ (prostacyclin) and E-type prostaglandins like $P_{G E_{1}}$, respectively. ${ }^{11}{ }^{12}$ These prostaglandins thus cause cAMP elevation and PKA activation, which suppresses pseudopod formation, $\mathrm{Ca}^{2+}$ fluxes, integrin activation, granule secretion and procoagulant activity. ${ }^{13-16}$

Genetic variation in the GNAS locus can affect the regulation of blood pressure and increase the risk of cardiovascular disease. ${ }^{17}$ Patients with several pathologies have been described with mutations in GNAS and altered Gsa function. ${ }^{18,19}$ In patients with the infrequent Albright hereditary osteodystrophy (AHO), a heterozygous inactivating mutation in the GNAS gene locus leads to hypofunction of the Gsa protein..$^{20-22}$ These patients are diagnosed with pseudohypoparathyroidism, type la (PHPla), due to impaired signalling of parathyroid hormone and other hormones. ${ }^{22,}$ ${ }^{23}$ Frequent characteristics are hormonal resistance, abnormal postural and facial features, brachydactyly and subcutaneous calcifications. Patients usually inherit the disease characteristics from their mother, as the maternal GNAS allele is preferentially expressed in specific tissues (paternal transmission without hormone resistance is classified as pseudopseudohypoparathyroidism, PPHP). ${ }^{24}$ In hematopoietic cells such as erythrocytes, both alleles are known to be expressed..$^{25}$ Earlier, Freson and colleagues have shown that the Gsa defect in patients with AHO (PHPla) can be identified by determining the low responsiveness of platelets to the IP receptor agonist iloprost (a prostacyclin mimetic) or the EP receptor agonist $\mathrm{PGE} 1{ }^{26}{ }^{26}$ The same group has also described a rare patient with compound heterozygous Gsa deficiency (different mutations in either allele), and $\sim 15 \%$ Gsa activity in platelets, which was accompanied by a prothrombotic phenotype, pointing to a role of this $G$ protein in human thrombosis and haemostasis. ${ }^{27}$

Recently, we have used protein mass spectrometry for the quantitative analysis of the proteome ${ }^{28}$ and the iloprost-induced phosphoproteome ${ }^{29}$ of human platelets, isolated from fresh blood donations. The high similarity of the (phospho) proteome of platelets from healthy individuals makes it possible to search for protein aberrations in platelets from patients with congenital deficiencies. ${ }^{30}$ Here, we have combined the workflows for a combined analysis of both the global proteome and the phosphoproteome using small platelet samples. In the present paper, we aimed to evaluate whether phosphoproteomics can be used to detect altered signalling in platelets from patients with established or suspected signalling defects. More specifically, as a proof-of-principle we elucidated the molecular alterations in the GsaAC-PKA pathway in platelets from a patient with AHO.

Using quantitative proteomics, we determined changes in the protein expression and protein phosphorylation patterns that are: (i) linked to defective Gsa activity, (ii) mediated by PKA and, (iii) explanatory for the changes in platelet functions. The data pave the way for pre-clinical identification of patients with assumed platelet abnormalities, such as patients with suspected AHO and related diseases based on the assessment of a modified signal transduction. 


\section{Materials and methods}

\section{Materials}

Iloprost was obtained from Bayer Schering Pharma (Leverkusen, Germany), and PGE from Fluka-Sigma Aldrich (Buchs, Switzerland). Horm type I collagen was purchased from Nycomed (Munich, Germany), thromboxane $A_{2}$ receptor agonist U46619 from Cayman Chemical Company (Ann Arbor MI, USA), 2-methylthioadenosine 5'-O-diphosphate (2-MeS-ADP) from Sigma Aldrich (Buchs, Switzerland). Convulxin was purified to homogeneity from crude snake venom. ${ }^{31}$ Proteinase-activated receptor-1 (PAR-1) agonist peptide SFLLRN was from Bachem (Bubendorf, Switzerland). Fluorescein isothiocyanate (FITC)-labelled antibody against P-VASP (phospho-Ser 239) was from nanoTools (Teningen, Germany), Alexa Fluor (AF)647labelled fibrinogen from Invitrogen Life Technologies (Bleiswijk, The Netherlands), FITC-labelled anti-CD62P mAb against P-selectin from Beckman Coulter (Marseille, France), and FITC-labelled PAC1 mAb against activated $\alpha_{1 \mathrm{lb}} \beta_{3}$ integrin from Becton Dickinson (San Jose CA, USA). The membrane probe DiOC 6 came from Anaspec (Reeuwijk, The Netherlands).

\section{Patients and controls}

Blood was obtained from healthy volunteers and from patients with established AHO, after full informed consent and in accordance with the Declaration of Helsinki. Experiments were approved by the local Medical Ethical Committee. Two familial related patients (mother I and son II) with Albright hereditary osteodystrophy (AHO) were investigated, diagnosed with symptomatic pseudohypoparathyroidism type la (OMIM: 103580), short stature and brachydactyly, who carried a heterozygous single nucleotide substitution in exon $1(c .1 A \rightarrow G)$ of the GNAS complex locus encoding $G$ sa. ${ }^{32}$ This mutation has been described to abolish expression of the mature Gsa protein, which in case of heterozygosity causes a partly reduced Gsa bioactivity in blood cells. ${ }^{22}$ Patient II, as a child, was only accessible for a single (remnant) blood sample. Hence, the majority of experiments were performed with blood from patient I. Blood donors did not use antiplatelet or anticoagulant medication for at least two weeks.

\section{Blood collection and platelet preparation and isolation}

For whole blood perfusion experiments, blood was collected into 0.1 volume of saline containing D-phenylalanyl-prolyl-arginyl chloromethyl ketone (PPACK, 40 $\mu \mathrm{M})$ and fragmin $(40 \mathrm{U} / \mathrm{mL}){ }^{33}$ For light transmission aggregometry, blood samples were collected into 0.1 volume of $129 \mathrm{mM}$ trisodium citrate. The latter were used for preparation of platelet-rich plasma (PRP) by centrifuging at $240 \times g$ for 15 minutes, and platelet-free plasma (PFP) by centrifuging twice at 2,630 $\times g$ for 10 minutes. Platelet 
counts were determined with a thrombocounter (Coulter Electronics; Woerden, The Netherlands).

For measurements with washed platelets, including proteomics analyses, blood samples were collected into 0.1 volume of acid-citrate glucose solution (ACD, 52 $\mathrm{mM}$ citric acid, $80 \mathrm{mM}$ trisodium citrate, $180 \mathrm{mM}$ D-glucose). ${ }^{34}$ PRP was obtained by centrifuging at $240 \times \mathrm{g}$ for 15 minutes. After addition of 0.066 volume of $A C D$, platelets were pelleted by centrifugation at $870 \times g$ for 15 minutes. The platelets were then resuspended in Hepes buffer pH $6.6(136 \mathrm{mM} \mathrm{NaCl}, 2.7 \mathrm{mM} \mathrm{KCl}, 10 \mathrm{mM}$ Hepes, $2 \mathrm{mM}$ $\mathrm{MgCl}_{2}$ and $0.1 \%$ D-glucose), while carefully excluding the bottom layer of red cells. After addition of 0.066 volume of $A C D$ and apyrase $(1 \mathrm{U} / \mathrm{mL})$, the platelets were washed by centrifugation at 2,000 $\times g$ for 5 minutes and resuspended in Hepes buffer $\mathrm{pH} 7.45$ ( $136 \mathrm{mM} \mathrm{NaCl}, 2.7 \mathrm{mM} \mathrm{KCl}, 10 \mathrm{mM}$ Hepes, $2 \mathrm{mM} \mathrm{MgCl}_{2}, 0.1 \%$ D-glucose), again by excluding the bottom layer of erythrocytes. Purity of the final platelet suspensions $\left(1-2 \times 10^{8} / \mathrm{mL}\right)$ was assessed with a thrombocounter and by microscopic analysis. Contamination of platelets with red blood cells was $<1: 15,000$ and with leukocytes was $<1: 20,000$.

\section{Light transmission aggregometry}

Aggregation of PRP $\left(3 \times 10^{8}\right.$ platelets $\left./ \mathrm{mL}\right)$ was measured with an automated Chronolog aggregometer (Havertown PA, USA). Samples were pre-incubated with vehicle (ethanol), $\mathrm{PGE}_{1}$ or iloprost for 4 minutes, and activated with $\mathrm{U}_{46619}(5 \mu \mathrm{M}), 2-\mathrm{MeS}$ ADP $(5 \mu \mathrm{M})$, SFLLRN $(15 \mu \mathrm{M})$, convulxin $(10 \mathrm{ng} / \mathrm{mL})$ or collagen $(5 \mu \mathrm{g} / \mathrm{mL})$ at $37^{\circ} \mathrm{C}$. Platelet aggregation rate was determined from the slopes of curves (\% transmission change per minute).

\section{CAMP measurements}

Levels of CAMP were measured, similarly as described before. ${ }^{35}$ Washed platelets (2 $\times 10^{8} / \mathrm{mL}$ ) in Hepes buffer $\mathrm{pH} 7.45$ were incubated with vehicle (ethanol) or $\mathrm{PGE}_{1}$, and samples of $200 \mu \mathrm{L}$ were taken at different time points. Reactions in the samples were stopped with ice-cold $70 \%$ ethanol, and the mixtures were frozen in liquid nitrogen. CAMP was measured in thawed samples using the Biotrak enzyme immunoassay system (Amersham Pharmacia Biotech; Eindhoven, The Netherlands).

\section{Thrombus formation and platelet activation under flow}

To measure whole blood thrombus formation, coverslips were coated with type I collagen and mounted into a parallel-plate flow chamber as previously described. ${ }^{36}$ Samples of PPACK-anticoagulated blood, pretreated for 4 minutes with vehicle, $\mathrm{PGE}_{1}(100 \mathrm{nM})$ or iloprost $(10 \mathrm{nM})$, were perfused over the collagen surface at a wall shear rate of $1,000 \mathrm{~s}^{-1}$ for 4 minutes. Where indicated, the membrane probe $\mathrm{DiOC}_{6}$ 
( $0.5 \mu \mathrm{g} / \mathrm{mL}$ ) was added to the blood as well as AF647-labelled fibrinogen $(16.5 \mu \mathrm{g} / \mathrm{mL}$ ) to measure platelet adhesion and integrin $\alpha_{11 b} \beta_{3}$ activation, respectively. ${ }^{36}$ Thrombi on coverslips were post-stained with FITC-labelled anti-CD62P mAb $(25 \mu \mathrm{g} / \mathrm{mL}){ }^{37}$ Brightfield differential interference contrast and confocal fluorescence images were taken, as described..$^{38}$ Microscopic images were analysed for surface area coverage using Image J (version 1.48g, US NIH; Bethesda MD, USA). ${ }^{36}$

\section{Flow cytometry}

For flow cytometry, samples of PRP $\left(3 \times 10^{8}\right.$ platelets $\left./ \mathrm{mL}\right)$ were incubated with vehicle (ethanol) or $\mathrm{PGE}_{1}$ for 4 minutes, and then activated with convulxin $(10 \mathrm{ng} / \mathrm{mL})$ or SFLLRN $(15 \mu \mathrm{M})$. After 20 minutes of agonist stimulation, P-selectin expression and integrin $\alpha_{1 \mathrm{lb}} \beta_{3}$ activation were determined with FITC-labelled anti-CD62 mAb and FITClabelled PAC1 mAb, respectively. ${ }^{34}$

For analysis of vasodilator-stimulated phosphoprotein (VASP) phosphorylation as a measure of Gs stimulation, samples of purified, washed platelets $\left(1 \times 10^{8} / \mathrm{mL}\right)$ were incubated with vehicle or iloprost $(0.5-10 \mathrm{nM})$ for indicated time, after which reactions were stopped with $2 \%$ formaldehyde (in filtered phosphate buffered saline with $0.2 \%$ bovine serum albumin). Fixed samples were centrifuged at 2,000 $\times g$ for 2 minutes, and pellets were washed twice with phosphate-buffered saline. The fixed and washed platelets were again resuspended in phosphate-buffered saline containing $0.1 \%$ saponin for membrane permeabilisation during 15 minutes. After addition of FITC-labelled anti-P-VASP Ab (1:1,000), samples were incubated for 30 minutes at room temperature. For all measurements (10,000 events/sample), a BD Accuri C6 flow cytometer was used (San Jose CA, USA).

\section{Sample preparation for proteome analysis}

For proteomics analyses, purified washed platelets $\left(1 \times 10^{8} / \mathrm{mL}\right)$ in Hepes buffer $\mathrm{pH}$ 7.45 were left untreated or treated with iloprost (1-10 nM) for 1 minute at $37^{\circ} \mathrm{C}$ (under non-stirring conditions). Reactions were stopped by addition of $50 \%$ lysis buffer (50 $\mathrm{mM}$ Tris, $150 \mathrm{mM} \mathrm{NaCl}, 1 \% \mathrm{SDS}, 1$ tablet Roche PhosStop/7 mL buffer, $\mathrm{pH} 7.8$ ), and incubation on ice. Lysed samples were directly stored at $-80{ }^{\circ} \mathrm{C}$ until further use. Parallel platelet samples were used for VASP-P determination by flow cytometry.

\section{Protein digestion and iTRAQ labelling for proteomics analysis}

Sample preparation, proteolytic digestion, quality control and iTRAQ labelling were based on previously described methods. ${ }^{28,29,39}$ Lysed samples of well purified platelets $\left(2 \times 10^{8}\right)$ were diluted to the same protein concentration (checked with a bicinchoninic acid protein assay kit; Pierce, Thermo-Fisher Scientific, Bremen, Germany). Cysteines were then reduced $\left(30\right.$ minutes, $56^{\circ} \mathrm{C}$ ) and free sulfhydryl groups were alkylated (30 
minutes, room temperature) with $10 \mathrm{mM}$ dithiothreitol and $30 \mathrm{mM}$ iodoacetamide, respectively. Afterwards, each subsample containing $100 \mu \mathrm{g}$ of protein was diluted 10 -fold with ice-cold ethanol and incubated for 1 hour at $-40{ }^{\circ} \mathrm{C}$. Samples were centrifuged for 30 minutes at $4{ }^{\circ} \mathrm{C}$ and $18,000 \times g$, and the supernatant was carefully removed. Next, precipitates were washed using $50 \mu \mathrm{L}$ of ice-cold acetone, followed by 15 minute centrifugation, as above. This step was repeated once.

Precipitated proteins were resolubilised in $6 \mathrm{M}$ guanidine hydrochloride, and digested in-solution with trypsin (Sequence grade modified, Promega) at a 1:20 enzyme : protein ratio, in a final concentration of $0.2 \mathrm{M}$ guanidine hydrochloride, $2 \mathrm{mM}$ $\mathrm{CaCl}_{2}$ and $50 \mathrm{mM}$ triethylammonium bicarbonate, TEAB $\left(14\right.$ hours, $\left.37^{\circ} \mathrm{C}\right)$. Digestion controls were performed, using a monolithic-RP, as previously described. ${ }^{28}$

Digests were individually labelled with iTRAQ 8-plex labels (113-119, 121). Therefore, samples were dried under vacuum, dissolved in iTRAQ 8-plex dissolution buffer ( $A B$ Sciex; Dreieich, Germany) and labelled according to the manufacturer's protocol. Four samples from patient I and 4 samples from a healthy control subject (i.e. unstimulated and stimulated with 1,2 , and $10 \mathrm{nM}$ iloprost, respectively) were pooled at 1:1 ratios, desalted by $C_{18}$ solid phase extraction (SPEC $C_{18} A R, 4$ mg bed; Agilent Technologies, Brussels, Belgium) and dried under vacuum.

\section{Platelet global proteome and phosphoproteome analysis}

The multiplexed iTRAQ pool of 8 platelet samples was analysed for determination of the global proteome and the phosphoproteome, after $\mathrm{TiO}_{2}$ enrichment. Hereto, $10 \%$ of the pooled mixture was pre-fractioned on a U3000 HPLC (Thermo-Fisher Scientific) by reversed-phase chromatography $\left(C_{18}\right.$ column; BioBasic-18 0.5 mm ID x 15 $\mathrm{cm} 5 \mu \mathrm{m}$ particle size $300 \AA$ A pore size, Thermo-Fisher Scientific) using a linear gradient ranging from $3-50 \%$ solvent $B$ (mobile phase $A: 10 \mathrm{mM}$ ammonium acetate $\mathrm{pH}$ 6.0, $B$ : $10 \mathrm{mM}$ ammonium acetate, $84 \%$ acetonitrile; $\mathrm{ACN}, \mathrm{pH} 6.0,75$ minutes) to obtain 20 concatenated fractions for LC-MS analysis.

For phosphopeptide analysis, $90 \%$ of the pooled iTRAQ sample was subjected to a $\mathrm{TiO}_{2}$-based phosphopeptide enrichment protocol, as described by the Larsen group with slight modifications. ${ }^{39}$ The sample was resuspended in $\mathrm{TiO}_{2}$ loading buffer (80 $\%$ ACN, $5 \%$ trifluoroacetic acid; TFA, $1 \mathrm{M}$ glycolic acid) and incubated with $\mathrm{TiO}_{2}$ beads for 10 minutes. The incubation was done first using a peptide to bead ratio of 1:6 and then using a ratio of 1:3. Next, the beads corresponding to both incubation steps were combined in one Eppendorf tube, washing and elution steps were conducted as described previously. ${ }^{40}$ In brief, $80 \%$ ACN, $1 \%$ TFA was used for washing step 1 and $10 \%$ ACN, $0.1 \%$ TFA for washing step 2. Phosphopeptides were eluted by incubation with 1 $\% \mathrm{NH}_{4} \mathrm{OH}$ for 10 minutes. Further, the eluates were acidified using formic acid $(\mathrm{pH}<2)$. To obtain a better phosphopeptide recovery, the enrichment procedure was repeated a second time with slight variations, as loading buffer $70 \%$ ACN, $2 \%$ TFA was used and $50 \%$ ACN, $0.1 \%$ TFA was used as washing buffer. Phosphopeptides were eluted as 
described above and further acidified. Finally, phosphopeptides were desalted using Oligo R3 microcolumns ${ }^{41}$ and fractionated on a U3000 RSLC system in hydrophilic interaction liquid chromatography (HILIC) mode (Polar phase TSKgel Amide-80; 150 $\mu \mathrm{m}$ ID $\times 15 \mathrm{~cm}$ length; $5 \mu \mathrm{m}$ particle size; $80 \AA$ Å pore size, Tosoh Bioscience, Tessenderlo, Belgium), using a binary gradient ranging from $10-35 \%$ solvent $B$ (solvent $A: 98 \% A C N$, $0.1 \%$ TFA; solvent B: $0.1 \%$ TFA) in 40 minutes (flow rate: $4 \mu \mathrm{L} /$ minute). A total of 9 fractions was collected for subsequent LC-MS analysis.

$\mathrm{RP}$ and HILIC fractions were individually analysed by nano-LC/MS-MS, using a U3000 RSLC system online-coupled to a Q-Exactive mass spectrometer (both ThermoFisher Scientific). Individual fractions were loaded onto a trap column (Acclaim PepMap100 $C_{18}$ trap column; $100 \mu \mathrm{m} \times 2 \mathrm{~cm}$ ) with $0.1 \%$ TFA (flow rate: $20 \mu \mathrm{L} /$ minute). This was followed by separation of peptides on the main column (PepMap100 $C_{18} ; 75$ $\mu \mathrm{m} \times 50 \mathrm{~cm})$, using a binary gradient ranging from $3-42 \%$ solvent B $(84 \% \mathrm{ACN}, 0.1 \%$ formic acid) in 145 minutes $\left(60^{\circ} \mathrm{C}\right.$, flow rate: $250 \mathrm{~nL} /$ minute). In the Q-Exactive, survey scans were acquired at resolution of 70,000 using an automatic gain control (AGC) target value of $3 \times 10^{6}$. MS/MS spectra of the top 15 most intense ions were acquired with a resolution of 17,500 , an isolation width of $2.0 \mathrm{~m} / \mathrm{z}$, a normalised collision energy of 35 , an AGC target value of $1 \times 10^{5}$ ions, a maximum injection time of $250 \mathrm{~ms}$ and a dynamic exclusion of $12 \mathrm{~s}$ with and underfill ratio of $10 \%$. The first fixed mass was set to $105 \mathrm{~m} / \mathrm{z}$. In order to compensate for the iTRAQ-induced increase of peptide charge states, reaction tubes with $10 \%$ ammonium water were placed in front of the ion source as described by Thingholm et al. ${ }^{42}$

Raw data were processed with Proteome Discoverer 1.4 (Thermo-Fisher Scientific). Data were searched against the Uniprot human database (August 2012; 20,232 target sequences) using Mascot and Sequest with the following settings: (i) trypsin as enzyme allowing two missed cleavages, (ii) iTRAQ 8-plex at N-termini and lysines (+ 304.2053 Da) and carbamidomethylation of Cys (+ 57.0214 Da) as fixed modifications, (iii) oxidation of Met $(+15.9949 \mathrm{Da})$ as variable modification, (iv) mass tolerances of $10 \mathrm{ppm}$ and to $0.02 \mathrm{Da}$ for MS and MS/MS, respectively. For HILIC fractions, phosphorylation of Ser/Thr/Tyr (+ 79.9663 Da) was selected as additional variable modification. False discovery rate (FDR) estimation on the level of peptide spectrum matches (PSM) was performed using the peptide validator node, filtering for $1 \%$ FDR (high confidence). The reporter ion quantifier node was used for iTRAQ reporter quantification. For global proteome quantification (RP fractions) only unique proteins quantified with at least 2 unique peptides were considered. For phosphoproteome quantification (HILIC fractions), phosphorylation site localisation was determined using phosphoRS ${ }^{43}$ and only phosphopeptides with phosphoRS site probabilities $>90 \%$ were considered as confident. 


\section{Data analysis for the global and phosphoproteome}

As Proteome Discoverer only provided 7 ratios for the 8 samples, an artificial 113/113 ratio was created and set to 1.0 per protein and all ratios were log2 transformed. For each channel a median over all protein ratios was calculated $\left(M D 1^{113-121}\right)$. Next, the median of all eight MD1 values was determined (MD2) to define normalisation factors (NF) per iTRAQ channel. These NF were used to compensate for individual systematic errors (i.e. unequal sample amounts derived from pipetting errors or inaccurate $B C A$ results) and to obtain normalised ratios (NR) per protein. Next, for each protein the individual NR were divided by the median (log2 subtraction) over all eight NR to obtain scaled normalised abundance values (NAV) for all proteins and channels. Using these NAVs ratios were determined between AHO patient and healthy control subject platelets for each condition (e.g. unstimulated) and log2-transformed.

Inter-individual variation was estimated by separate analysis of samples (unstimulated platelets) from pairs of subjects over all proteins; after log2 transformation this gave a mean SD of 0.15 (range 0.11-0.20, $n=4$ ). Boundaries for relevant changes in the patient were set at $2 \times \mathrm{SD}(2 \times 0.16)$ in comparison to median abundance ratios, for up- or down-regulation per protein. Inter-individual variation over all phosphopeptides was estimated from separate analysis of resting platelets from pairs of subjects, this gave a mean log2 SD of 0.39 (range 0.34-0.42, $n=4$ ). Boundaries for relevant changes were again set at $2 \times \mathrm{SD}(2 \times 0.39)$ in comparison to median abundance ratios, for up- or down-regulation per phosphopeptide.

Furthermore, for determination of confident phosphorylation sites at the peptide level, a ready-to-use Excel macro provided by Mechtler lab (http://ms.imp. ac.at/?goto=phosphors) was used. Data normalisation was performed as described above, using the NF calculated from the global proteome. Next, for each PSM NAVs were calculated, as mentioned above for the global proteome. PSMs representing the same protein, phosphopeptide sequence and phosphorylation site were grouped and the average NAVs were calculated per iTRAQ channel. Finally ratios were calculated as mentioned above and log2-transformed.

Normal reference values of phosphoproteome and determination of PKA phosphorylation sites

Phosphorylation data were compared with a reference dataset of iloprostinduced changes in protein phosphorylation of healthy control platelets $(2,700$ phosphopeptides, of which 299 regulated by iloprost), as recently published. ${ }^{29}$ lloprost-regulated phosphopeptides were defined as those responsive to 1 minute treatment with 2 or $5 \mathrm{nM}$ iloprost. A 3-point scale was used ( $1=$ up, $0=$ unchanged, -1 = down). Consensus sites for PKA-induced phosphorylation were defined as before, ${ }^{29}$ using the GPS2.1 algorithm for kinase consensus sequence prediction. ${ }^{44}$ Classification of proteins to 21 platelet function classes ${ }^{30}$ was based on GeneOntology and Uniprot descriptions. 


\section{Statistics}

Experimental data are represented as means \pm SD. Statistical significance of differences between groups was determined using the independent samples $t$ test. $P$-values $<0.05$ were considered significant.

\section{Results and Discussion}

Impaired Gs $\alpha$-mediated regulation of platelet activation for two patients with $\mathrm{AHO}$

Congenital deficiency in Gsa signalling activity, as in patients with mutations in the GNAS gene cluster, can be determined in platelets from the inhibitory effect of $\mathrm{PGE}_{1}$ or iloprost on collagen-induced platelet aggregation. ${ }^{26}$ Both prostaglandin derivatives bind to Gsa-coupled receptors, and thus stimulate adenylate cyclase to produce the key platelet-inhibiting second messenger CAMP. ${ }^{10}$ We used this assay to confirm a platelet phenotype in two related AHO patients with a heterozygous deleterious mutation in the GNAS gene cluster and pseudohypoparathyroidism type la, designated as patient I (mother) and patient II (child). In platelets from 10 healthy control donors, platelet aggregation in response to collagen was inhibited by the Gsa-stimulating $\mathrm{PGE}_{1}$ in a dose-dependent manner (Fig. $1 \mathrm{~A}$ ). In platelets from the two patients with $A H O$, this inhibition by $\mathrm{PGE}_{1}$ was greatly diminished (Fig. 1B, C). Yet, high concentrations of $\mathrm{PGE}_{1}>100 \mathrm{nM}$ did abrogate the collagen-induced aggregation of the patients platelets.

A

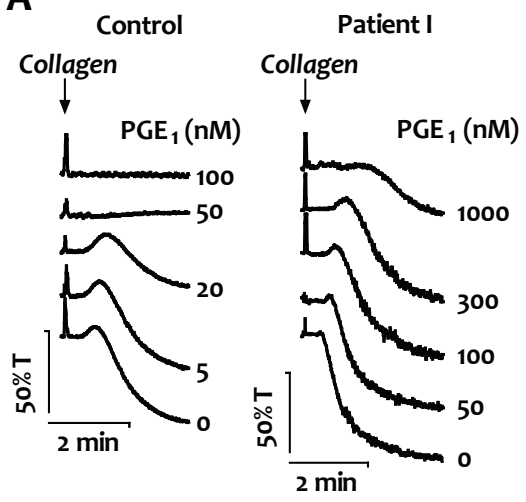

B

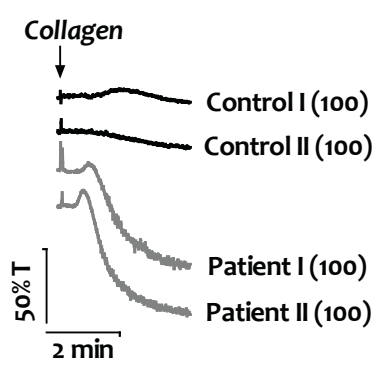

C

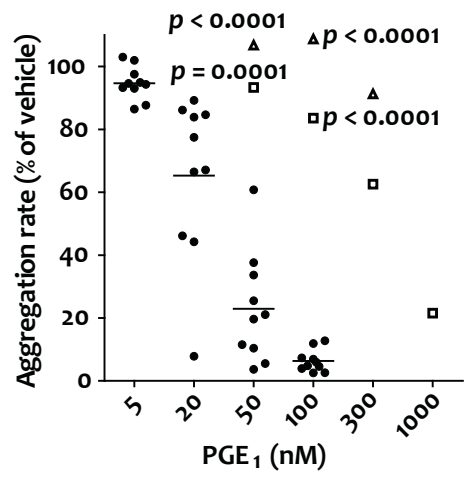

Figure 1. Impaired Gsa-mediated inhibition of platelet aggregation in patients with AHO. PRP was pretreated with vehicle or indicated concentration of $\mathrm{PGE}_{1}$ (in $\mathrm{nM}$ ) for 4 minutes, and activated with collagen $(5 \mu \mathrm{g} / \mathrm{mL})$. (A) Representative aggregation traces of platelets from a healthy subject (day control) and patient I. (B) Inhibition of platelet aggregation by $100 \mathrm{nM} \mathrm{PGE} \mathrm{E}_{1}$ for two control subjects (day controls) and $\mathrm{AHO}$ patients I and II. (C) Platelet aggregation rate at various doses of $\mathrm{PGE}_{1}$ for ten healthy controls (black dots) and two AHO patients (patient I; open square, patient II; open triangle). Per subject, platelet aggregation without $\mathrm{PGE}_{1}$ was set at $100 \%$. Data are means \pm SD ( $n=10$ controls); ${ }^{*} p<0.05$. 
In platelets from control donors, $\mathrm{PGE}_{1}$ inhibited in a dose-dependent way the aggregation induced by several conventional agonists, although at different efficacy. The $I C_{50}$ of $P G E_{1}$ to suppress platelet aggregation increased in the order of thrombinreceptor activating peptide SFLLRN ( $\left.15 \mathrm{nM} \mathrm{PGE}_{1}\right)<$ thromboxane $\mathrm{A} 2$ analogue $\mathrm{U}_{46619}$, collagen $(35 \mathrm{nM})<2$-MeS-ADP $(55 \mathrm{nM})<$ glycoprotein VI (GPVI) agonist, convulxin (85 $\mathrm{nM}$ ) (Supplemental Fig. $1 \mathrm{~A}$ ). Markedly, for the platelets from patient $I$, this $I C_{50}$ was $>100 \mathrm{nM}$ with all agonists used. In control platelets, in agreement with earlier data, ${ }^{35} 50$ and $100 \mathrm{nM} \mathrm{PGE}_{1}$ induced a transient, 2.7- and 5.5-fold increase in CAMP, respectively. The platelets from patient I showed a lower CAMP increase with $\mathrm{PGE}_{1}$ (Supplemental Fig. 1B, C). Taken together, these data indicated that the platelets from both AHO patients responded in a less effective way to the Gsa-stimulating agonist $\mathrm{PGE}_{1}$ to

A
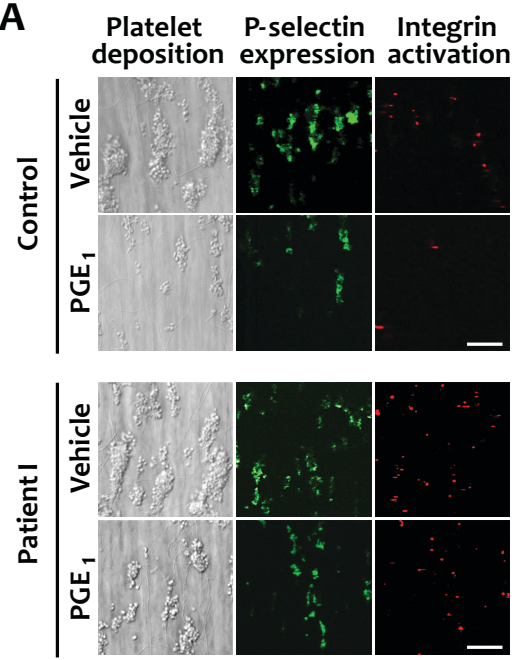

B

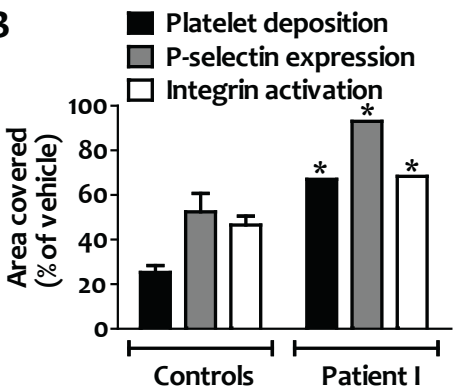

C
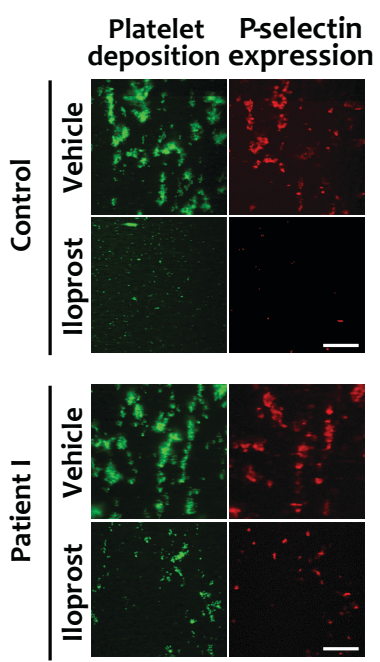

D

Platelet deposition

P-selectin expression

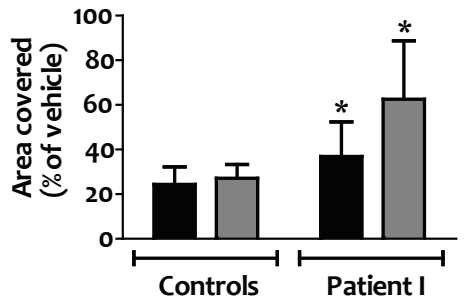

Figure 2. Impaired Gsa-mediated inhibition of thrombus formation under flow in AHO patient. Thrombus formation was assessed by whole blood perfusion over collagen at shear rate of 1,000 $\mathrm{s}^{-1}$. Thrombi were stained after 4 minutes, and brightfield and fluorescence images recorded. Blood samples were pretreated with vehicle, $\mathrm{PGE}_{1}(100 \mathrm{nM})$ or iloprost $(10 \mathrm{nM})$, as indicated. (A) Representative images of platelet deposition, P-selectin expression (FITC anti-CD62P mAb) and integrin activation (AF647fibrinogen) with blood from healthy controls and from patient I (bars, $20 \mu \mathrm{m})$. (B) Effect of PGE $\mathrm{B}_{1}$ on thrombus formation (\% surface area covered). (C) Representative images of platelet deposition (DiOC 6 ) and integrin activation (AF647-fibrinogen) with blood from healthy controls and patient I. (D) Effect of iloprost on thrombus formation (\% surface area covered). Means \pm SD ( $n=3$ control donors); ${ }^{*} p<0.05$. 
reduce aggregation compared to control platelets.

Impaired Gs $\alpha$-dependent inhibition of thrombus formation with blood from AHO patient

We measured thrombus formation on a collagen surface under high shear flow conditions, in order to assess the effect of Gsa stimulation in flowing whole blood. ${ }^{37}$ Without $\mathrm{PGE}_{1}$, blood from control donors and patient I gave highly similar, large thrombi of aggregated platelets, which stained highly positive for P-selectin and activated integrin $\alpha_{1 \mathrm{~b}} \beta_{3}$, as determined with specific fluorescent probes (Fig. 2A). However, with $\mathrm{PGE}_{1}$ added to the blood, thrombus formation (platelet deposition) and P-selectin expression - and to a lesser extent integrin activation - were more reduced in the blood samples from control subjects than in the patient blood (Fig. 2A, B). Similar results were obtained by addition of another, stronger Gsa-stimulating agent, i.e. iloprost. In the presence of iloprost, platelet deposition and P-selectin expression decreased by $\sim 75 \%$ with blood from control donors, whereas these parameters decreased by $\sim 40 \%$ with blood from patient I (Fig. 2C, D).

Flow cytometry was used to determine P-selectin expression (as a marker of platelet secretion) and integrin $\alpha_{1 \mathrm{Il}} \beta_{3}$ activation. In control platelets stimulated with the PAR1 agonist SFLLRN and the GPVI collagen receptor agonist convulxin, PGE $_{1}$ dose-dependently suppressed either activation process. Markedly, with platelets from patient I, the dose-response curve was shifted to the right for all agonists, when compared to the curves of control platelets (Supplemental Fig. 2A-D). Thus, the hypo-responsiveness to Gsa-stimulating agents of the patient's platelets included secretion, integrin activation and thrombus formation.

\section{Subtle changes in overall proteome of platelets from $\mathrm{AHO}$ patient}

Using a combined approach of quantitative proteomics and phosphoproteomics, we investigated on a molecular level the altered PKA-dependent signalling pathway in Gsa-stimulated AHO platelets. From healthy control donors and patient I, PRP and well-purified washed platelets were obtained. The other patient II was not accessible for new blood donations. Iloprost at $5 \mathrm{nM}$ substantially suppressed the rate of platelet aggregation for control donors, but not for patient I (Fig. 3A, B). Parallel samples of washed platelets were used for determination of VASP phosphorylation at Ser-239, as an established PKA-dependent phosphorylation event, ${ }^{45}$ and for proteomics workup. Samples of $2 \times 10^{8}$ platelets were stimulated with a range of iloprost concentrations (0.5-10 nM) for 1 minute, to allow direct comparison with the earlier described iloprostinduced phosphoproteome. ${ }^{29}$

In purified platelets from a control donor (day control), iloprost at $\geq 0.5 \mathrm{nM}$ caused detectable VASP phosphorylation, whereas $\geq 2 \mathrm{nM}$ iloprost was required for phosphorylation of the platelets from patient I (Fig. 3C, D). Based on this result, (phospho)proteomics analysis was performed with control and patient platelets 
A

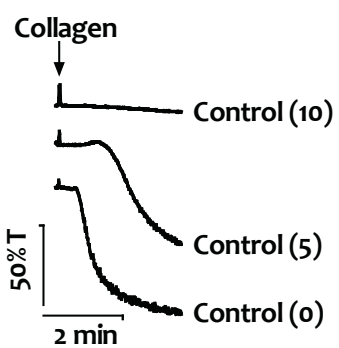

C

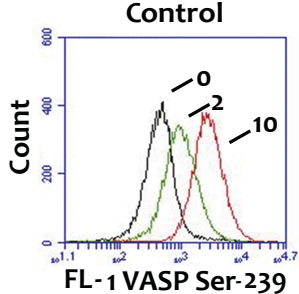

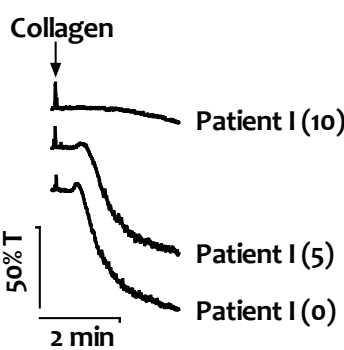

Patient I

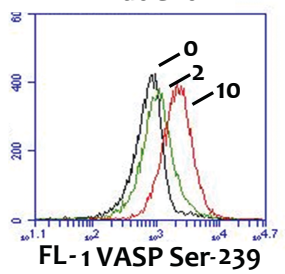

B
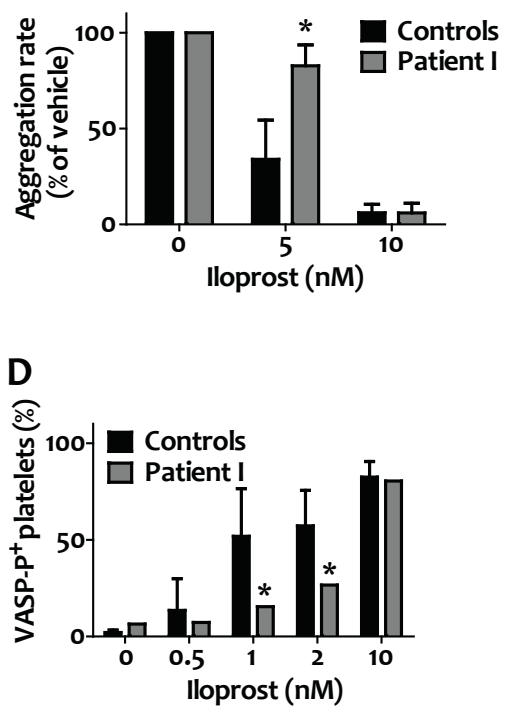

Figure 3. Impaired Gsa-mediated iloprost-induced responses of AHO platelets. Platelets in PRP were pretreated with vehicle or indicated concentration of iloprost (in $\mathrm{nM}$ ) for 4 minutes, and activated with collagen $(5 \mu \mathrm{g} / \mathrm{mL})$. (A) Platelet aggregation traces from a healthy subject (day control) and from patient I. (B) Inhibition of platelet aggregation rate by 5-10 nM iloprost for eight control subjects and patient I. (C-D) Purified washed platelets were preincubated with iloprost (0-10 nM), fixed, and stained with FITC anti-P-VASP Ab. Shown are flow cytometric histograms of VASP phosphorylation after iloprost treatment for control I and patient I (C), and averaged data from 8 controls and patient I (D). Means \pm SD ( $n=8$ controls); ${ }^{*} p<0.05$.

treated with 0, 1, 2 or $10 \mathrm{nM}$ iloprost. Eight samples (4 of control, 4 of patient) were analysed simultaneously using iTRAQ labelling (see Methods). Detailed analysis of the global proteome provided quantitative information on 1,206 unique proteins of platelets from the patient and control subject (Supplemental Datasheet S1). Plotting the log2 transformed ratios as a function of their frequency resulted in a normal distribution profile. Similar comparisons of data from 4 pairs of subjects gave an SD (log2 transformed) of 0.158 (see Methods). Considering a deviance of $2 \times 0.158$ from the median as a relevant change in protein abundance, levels outside of this range were tagged as altered in the patient samples (Fig. 4A). Hence, we concluded that the global proteome was no more than slightly changed in platelets from the AHO patient. Of the $19(1.6 \%)$ down-regulated proteins and the $44(3.7 \%)$ up-regulated proteins, markedly changed were the regulatory subunits RII $\beta$ (PRKAR2B) and RI $\beta$ (PRKAR1B) of PKA, forming the PKA-II and PKA-I holoenzymes. ${ }^{8}$ These were $38 \%$ and $19 \%$ reduced in the patient's platelets, respectively (Supplemental Datasheet S1). The GNAS product, Gsa as integral membrane protein with low expression level was not detected. 


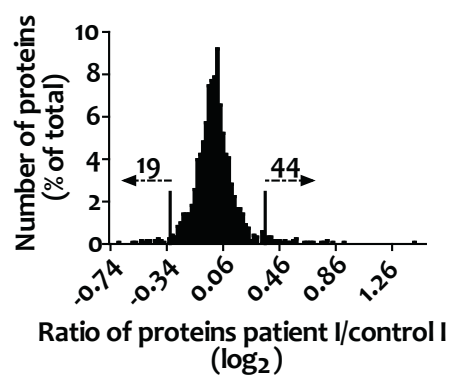

Figure 4. Alterations in global proteome of platelets from patient with AHO. Global proteomics analysis providing ratioed quantitative information on 1,206 unique proteins of platelets from patient I and control I (day control subject). Histogram of normalised abundance ratios of patient I/control I per identified protein. Ratios were plotted after $\log 2$ transformation; with values outside the range of median (-0.001) $\pm 2 \times 0.158$ considered as relevant differences. Indicated are numbers of proteins with up- or downregulation in the patient's platelets. For pairs of healthy control subjects $(n=4)$, this analysis by default yielded $\sim 2.3 \%$ of proteins in either category.

Identification of iloprost-induced changes of phosphorylation sites in platelets

Phosphoproteomics analysis of the patient and control iTRAQ-labelled samples was performed after $\mathrm{TiO}_{2}$ enrichment. In total, we could quantify 3,457 phosphopeptides by mass spectrometry, corresponding to 1,356 unique proteins (Supplemental Datasheet S1). These data present a $28 \%$ increase in comparison to the database described earlier of 2,700 phosphopeptides (comprising 299 proteins regulated by iloprost) in platelets from healthy controls. ${ }^{29}$

From comparisons between the phosphoproteome of resting platelets of pairs of control subjects, we set boundaries to determine relevant changes for each of the 3,457 phosphopeptides (deviant >2 $\times 0.385$, log2 transformed). In control platelets, iloprost at 1, 2, and $10 \mathrm{nM}$ thus increased the phosphorylation of 53, 71 and 239 phosphopeptides, respectively (Fig. 5 A, B). Of the 239 positively regulated phosphopeptides at $10 \mathrm{nM}$, more than half have not been identified before (56\%, white shading in Fig. 5A), ${ }^{29}$ and the majority contains consensus PKA phosphorylation sites (66 \%, black shading in Fig. 5B). We also noted increased phosphorylation of VASP at Ser-239, thus confirming the flow cytometric data (Fig. 3C, D).

On the other hand, in the control platelets, iloprost also decreased the phosphorylation of multiple phosphopeptides (Fig. 5C, D). These events are most probably indirect changes by the Gsa-AC-PKA pathway, as the majority of the negatively regulated phosphopeptides was not identified as iloprost-responsive (Fig. 5C) and lacks consensus PKA phosphorylation sites (Fig. 5D). For the complete lists of iloprost-regulated peptides at different concentrations, see Supplemental Datasheet S1.

The listed 239 positively regulated phosphopeptides corresponded to 175 unique proteins. Assignment of these to platelet function classes (Fig. $5 \mathrm{E}$ ) indicated that the iloprost-induced changes especially involved proteins implicated in: Cytoskeleton 
actin-myosin (14 proteins), Membrane \& protein trafficking(10×), Membrane receptors \& channels (12x), Protein kinases \& phosphatases (25x), Cytoskeleton receptor-linked (12x), Signalling \& adapter proteins (28x), and Small GTPases \& regulators (16x).

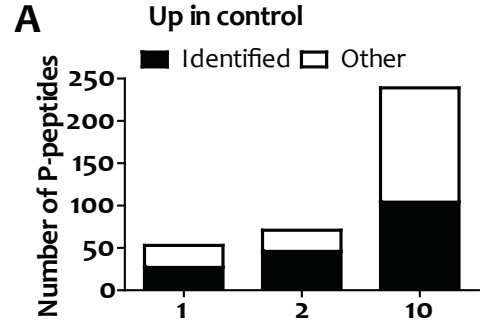

B

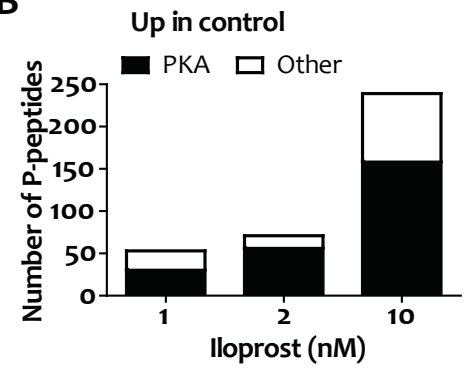

E

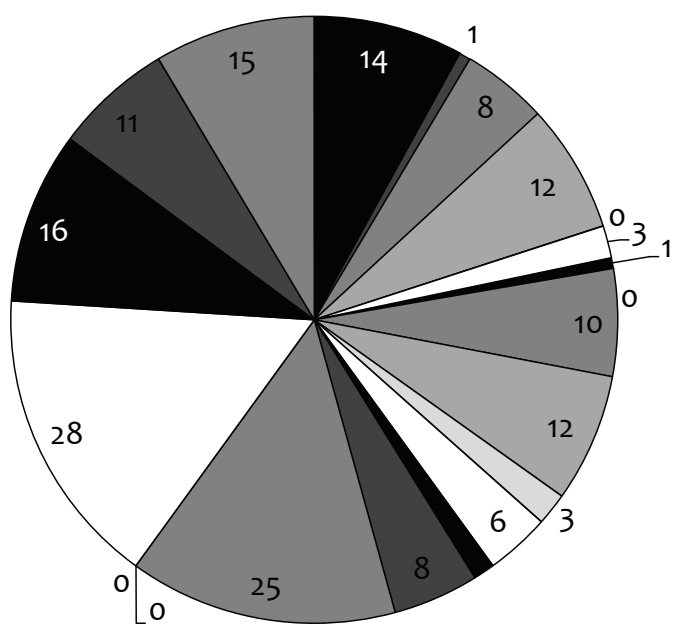

D
C Down in control
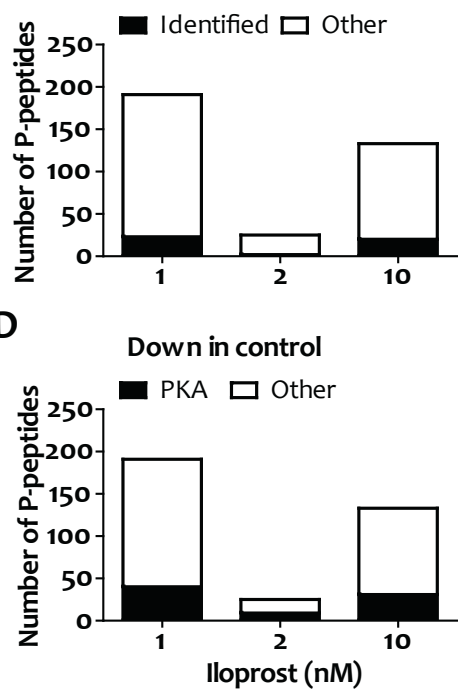

1. Cytoskeleton actin-myosin (14)

$\square$ 2. Cytoskeleton intermediate (1)

ㄱ. Cytoskeleton microtubule (8)

$\square$ 4. Cytoskeleton receptor-linked (12)

$\square$ 5. Endosome proteins (0)

$\square 6$. ER \& Golgi proteins (3)

7. Glucose metabolism (1)

$\square$ 8. Lysosome \& peroxisome proteins (0)

口9. Membrane \& protein trafficking (10)

$\square$ 10. Membrane receptors \& channels (12)

$\square$ 11. Mitochondrial proteins (3)

$\square$ 12. Other metabolism (6)

13. Other nuclear proteins (2)

$\square$ 14. Proteasome (8)

$\square$ 15. Protein kinases \& phosphatases (25)

$\square$ 16. Protein processing (0)

$\square$ 17. Secretory proteins (0)

$\square$ 18. Signalling \& adapter proteins (28)

-19. Small GTPases \& regulators (16)

घ20. Transcription \& translation (11)

ธ 21. Unknown (15)

Figure 5. Iloprost-induced, PKA-mediated protein phosphorylation changes in control platelets. Indicated are numbers of phosphopeptides found to be positively regulated $(\mathbf{A}, \mathbf{B})$ or negatively regulated (C, D) by 12 , or $10 \mathrm{nM}$ iloprost (outside range of median $\pm 2 \times 0.385$ ). Bars indicate total numbers and expected numbers (black shading). (A, C) black = number previously identified. ${ }^{29}$ (B, D) black = number with positive PKA score. (E) Distribution of 175 positively regulated phosphoproteins (10 nM iloprost) over different platelet function classes. 
Identification of altered Gs $\alpha$-dependent phosphorylation sites in AHO platelets

Subsequently, we evaluated differences in iloprost-induced phosphorylation between the patient and control platelets. Based on the same criteria for relevant changes in the 1,356 identified phosphopeptide ratios, we found similar numbers of positively and negatively regulated phosphopeptides in platelets from both subjects (Supplemental Fig. 3A). However, histograms of phosphopeptide ratios of iloprost-stimulated versus unstimulated platelets showed a smaller curve shift for the patient platelets than for the control platelets (Supplemental Fig. 3B, C).

In a more targeted approach, we compared the previously identified 239 positively regulated phosphopeptides (control platelets, $10 \mathrm{nM}$ iloprost, effect range 23.13-1.85), with a set of 100 non-regulated phosphopeptides (idem, effect range 0.99-1.00), and the 133 identified negatively regulated phosphopeptides (idem, effect range 0.58 0.09). For a complete list, see Supplemental Datasheet $\mathrm{S1}$. For each of these groups per iloprost concentration, we determined the differences in phosphopeptide abundance between the patient and control platelets. Strikingly, in the patient platelets, the overall abundance of the positively regulated phosphopeptides was substantially lower (Fig. 6A, $p<0.0001$ ). At 1, 2 and $10 \mathrm{nM}$ iloprost, levels of 32, 163 and 140 of the 239 phosphopeptides were decreased in the patient platelets (Fig. 6B). In contrast, no overall change was observed in the set of 100 non-regulated phosphopeptides (Fig. $6 C, D, p=0.733$ ). For the negatively regulated phosphopeptides, there was a small but significant increase in abundance in the patient platelets (Fig. $6 \mathrm{E}, p<0.0001$ ). This increase concerned the majority of the down-regulated phosphopeptides (Fig. 6F).

For each of the 239 positively iloprost-regulated phosphopeptides, we then compared the changes in relative abundance levels between patient and control platelets. In total, for all three iloprost concentrations, we identified relevant changes in 180 of 239 regulated phosphopeptides (143 of the 175 regulated unique proteins), which in $84 \%$ of the cases concerned a decreased abundance in the patient platelets (top 95; Supplemental Table 1). Taken together, this pointed to a systematic lowering of iloprost-induced phosphorylation in platelets from the AHO patient in the majority of the newly recognised phosphopeptides regulated by iloprost.

Functional and diagnostic relevance of altered Gs $\alpha$-AC-PKA phosphorylation in AHO platelets

The 180 changed phosphopeptides corresponded to 143 unique platelet proteins. Mapping these 143 proteins to function classes (Fig. 6G) indicated changes in the patient platelets especially of proteins implicated in: Cytoskeleton actin-myosin (13 proteins), Membrane \& protein trafficking ( $9 \times$ ) Membrane receptors \& channels (10x), Protein kinases $\&$ phosphatases (17x), Cytoskeleton receptor-linked (11x), Signalling \& adapter proteins $(23 \times)$, and Small GTPases \& regulators (15x). Accordingly, the majority of phosphorylation events in these classes that were identified as iloprost- 
upregulated appeared to be altered in the patient platelets.

Closer examination of the list indicated 19 proteins, which earlier have been identified as potential PKA targets, because of their immediate responses to low iloprost concentrations. The list contains multiple proteins that are implicated in key signalling pathways to platelet activation, ${ }^{9,}{ }^{10}$ suggesting that

A

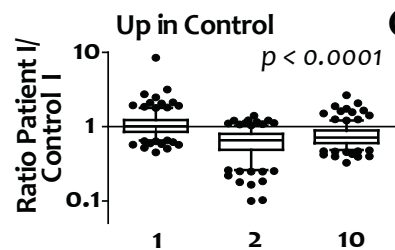

C

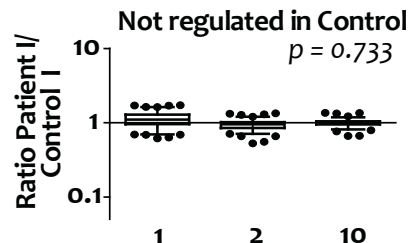

Down in Control

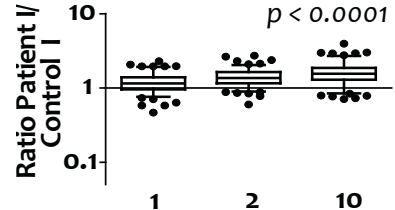

B

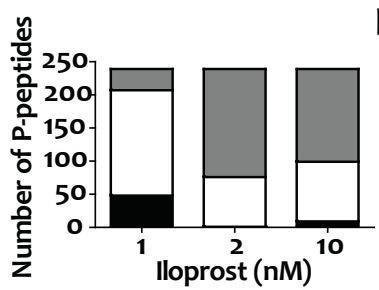

D $气$

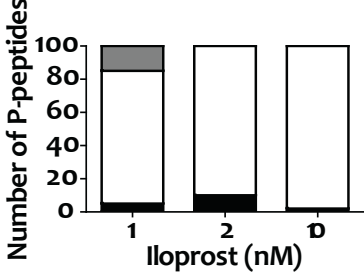

$\mathbf{F}$

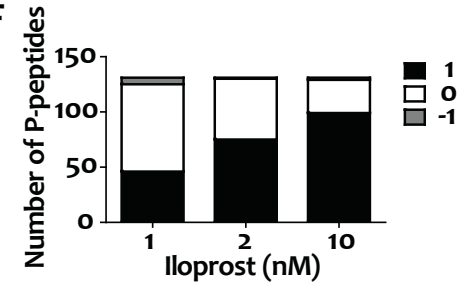

G

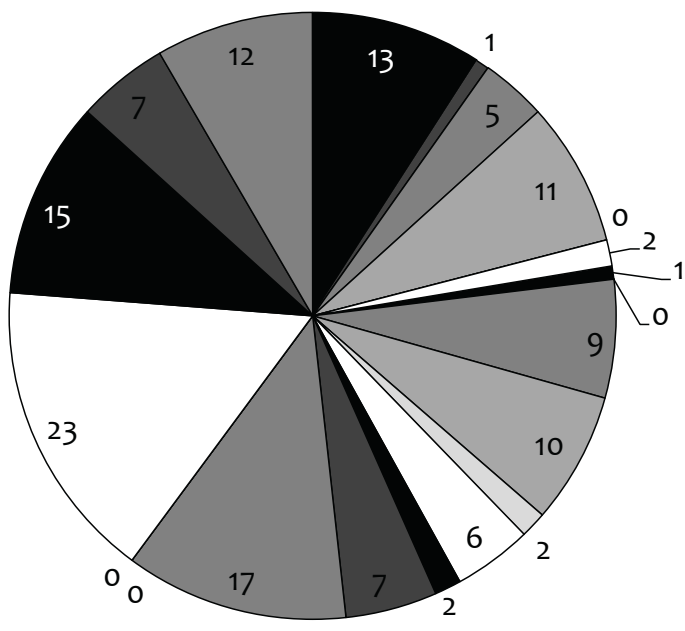

1. Cytoskeleton actin-myosin (13)

$\square$ 2. Cytoskeleton intermediate (1)

口3. Cytoskeleton microtubule (5)

$\square$ 4. Cytoskeleton receptor-linked (11)

प5. Endosome proteins (0)

口6. ER \& Golgi proteins (2)

7. Glucose metabolism (1)

$\square$ 8. Lysosome \& peroxisome proteins (0)

口9. Membrane \& protein trafficking (9)

$\square$ 10. Membrane receptors \& channels (10

$\square$ 11. Mitochondrial proteins (2)

$\square$ 12. Other metabolism (6)

13. Other nuclear proteins (2)

$\square$ 14. Proteasome (7)

口15. Protein kinases \& phosphatases (17)

$\square$ 16. Protein processing (0)

$\square$ 17. Secretory proteins (0)

$\square$ 18. Signalling \& adapter proteins (23)

19. Small GTPases \& regulators (15)

口20. Transcription \& translation (7)

घ21. Unknown (12)

Figure 6. Changes in iloprost-induced protein phosphorylation changes in AHO platelets. Normalised abundance ratios were obtained per phosphopeptide for platelets from patient I/control I. Data are separated according to regulation by iloprost: (A, B) 239 positively regulated, (C, D) 100 non-regulated, and (E, F) 131 negatively regulated phosphopeptides. (A, C, E) Box plots of means and $90 \%$ confidence intervals per class. Note the overall reduced phosphorylation in the patient's platelets at 2 and $10 \mathrm{nM}$ iloprost in panel A. P values over all iloprost concentrations. (B, D, F) Bar graphs indicating the direction of changed ratios in patient platelets per phosphopeptide: -1 = reduced in patient, $0=$ unchanged in patient, +1 = increased in patient. (G) Distribution of 143 phosphoproteins with altered phosphorylation in patient over different platelet function classes. 
at least several of these change in activity after phosphorylation at the identified iloprost-responsive sites. These include phospholipase $C-\beta$ isoforms (PLCB2, PLCB3); type $3 \mathrm{~A}$ phosphodiesterase (PDE3A); vasodilator-stimulated phosphoprotein $(\mathrm{VASP})^{8}$; the inositol phosphate receptor regulator IRAG ${ }^{29}$; and several members of the mitogen-activated protein kinase cascades (MINK1, MAP2K4, MAP3K2). Proteins that may control platelet shape change via altered phosphorylation of cytoskeletal components are in particular: LIM and $\mathrm{SH}_{3}$ domain protein 1 (LASP1); myosin light-chain kinase (MYLK); a myosin isoform (MYO9B); cortactin (CTTN); and the small GTPase-regulating proteins, Rho-GTPAse-activating protein 6/9 (ARHGAP6, ARHGAP9) and Rac/Cdc42 guanine nucleotide exchange factor (GEF) 6 (ARHGEF6) ${ }^{46}$. Proteins that control platelet integrin $\alpha_{111} \beta_{3}$ activation and hence platelet aggregation e.g. via altered phosphorylation are especially: the Rap1 controlling proteins, CalDAGGEFI (RASGRP2) ${ }^{47}$; and Rap1 GTPase-activating protein (RAP1GAP2) actin-linked proteins kindlin-3 (FERMT3), talin-1 (TLN1) and filamin A (FLNA). SNAREbinding proteins like tomosyn (STXPB5) are known to regulate granular secretion. ${ }^{46}$

Altogether, our data reveal new iloprost-regulated phosphorylation changes in key protein kinases and protein phosphatases involved in platelet function. We hypothesise that altered activity of several of these kinases and phosphatases can explain the changes in phosphorylation profile in AHO platelets. The data thereby suggest PKA-dependent regulation of one or more (serine/threonine) phosphatases, but this remains speculative, as only very little is known of the regulatory mechanisms of these phosphatases in platelets. Further confirmation of the precise molecular events will require new genetic approaches, such as the generation of site-directed mutations of specific proteins using CRISP-R technologies.

\section{Conclusions}

Our findings provide for the first time a detailed description of the altered Gsadependent functional responses of AHO platelets, i.e. integrin activation, granule secretion, activation, aggregation, and thrombus formation. Since platelet function inhibition via Gsa involved suppression of alpha granule secretion and integrin $\alpha_{11 b} \beta_{3}$ activation to a similar extent, these are likely mediated via a set of common downstream signalling events. Furthermore, by sensitive combined (phospho)proteomics analysis, we have identified novel 180 phosphorylation events of the Gsa-AC-PKA axis that can be used for the assessment and diagnosis of altered signal transduction in $\mathrm{AHO}$ and related diseases. From the viewpoint that platelet proteomics can act as an important tool for determining the role of platelets in health and disease, ${ }^{30}$ the present (phospho)proteomics analysis is useful not only for diagnostic purposes, but also for understanding the molecular events regulating platelet inhibition via the key Gsa-AC-PKA signalling pathway. 


\section{Acknowledgements}

This research was supported by the Centre for Translational Molecular Medicine (INCOAG), The Cardiovascular Centre Maastricht, and the Ministerium für Innovation, Wissenschaft und Forschung des Landes Nordrhein-Westfalen.

\section{Authorship contributions}

JWMH and RPZ were the principal investigators and take primary responsibility for the paper; FS, FAS, MAHF, NJAM and FB performed analytical work; CTRMS and IMLWK-K recruited the patients; FS, FAS, MAHF and PEJM performed statistical analysis; FS, FAS, AS, JWMH and RPZ coordinated the research; FS, FAS, JWMH and RPZ wrote the paper.

\section{Conflict of interest}

The authors declare no conflicts of interest.

\section{References}

1. Freson K, Hoylaerts MF, Jaeken J, Eyssen M, Arnout J, Vermylen J, Van Geet C. Genetic variation of the extra-large stimulatory $\mathrm{G}$ protein $\alpha$-subunit leads to $\mathrm{Gs}$ hyperfunction in platelets and is a risk factor for bleeding. Thromb Haemost. 2001;86:733-738.

2. Kozasa T, Itoh H, Tsukamoto T, Kaziro Y. Isolation and characterization of the human Gsa gene. Proc Natl Acad Sci USA. 1988;85:2081-2085.

3. Peters J, Williamson CM. Control of imprinting at the Gnas cluster. Epigenetics. 2007;4:207-213.

4. Wettschureck N, Offermanns S. Mammalian G proteins and their cell type specific functions. Physiol Rev. 2005;85:1159-1204.

5. Tasken K, Aandahl EM. Localized effects of CAMP mediated by distinct routes of protein kinase A. Physiol Rev. 2004;84:137-167.

6. Scott JD. Cyclic nucleotide-dependent protein kinases. In: Intracellular Messengers (Taylor, CW, ed) Pergamon Press, Oxford, UK. 1993:137-166.

7. Raslan Z, Naseem KM. The control of blood platelets by CAMP signalling. Biochem Soc Trans. 2014;42:289-294.

8. Smolenski A. Novel roles of CAMP/cGMP-dependent signaling in platelets. J Thromb Haemost. 2012;10:167-176.

9. Versteeg HH, Heemskerk JW, Levi M, Reitsma PS. New fundamentals in hemostasis. Physiol Rev. 2013;93:327-358.

10. Swieringa F, Kuijpers MJ, Heemskerk JW, Van der Meijden PE. Targeting platelet receptor function in thrombus formation: the risk of bleeding. Blood Rev. 2014;28:9-21.

11. Paul BZ, Ashby B, Sheth SB. Distribution of prostaglandin IP and EP receptor subtypes and isoforms in platelets and human umbilical artery smooth muscle cells. Br J Haematol. 1998;102:1204-1211.

12. Zhang L, Wu J, Ruan KH. Solution structure of the first intracellular loop of prostacyclin receptor and implication of its interaction with the C-terminal segment of Gas protein. Biochemistry. 2006;45:1734-1744.

13. Feijge MA, Ansink K, Vanschoonbeek K, Heemskerk JW. Control of platelet activation by cyclic 
AMP turnover and cyclic nucleotide phosphodiesterase type-3. Biochem Pharmacol. 2004;67:15591567.

14. Siess W, Lapetina EG. Functional relationship between cyclic AMP-dependent protein phosphorylation and platelet inhibition. Biochem J. 1990;271:815-819.

15. Vanschoonbeek K, Feijge MA, Van Kampen RJ, Kenis H, Hemker HC, Giesen PL, Heemskerk JW. Initiating and potentiating role of platelets in tissue factor-induced thrombin generation in the presence of plasma: subject-dependent variation in thrombogram characteristics. J Thromb Haemost. 2004;2:476-484.

16. Yan R, Wang Z, Yuan Y, Cheng H, Dai K. Role of cAMP-dependent protein kinase in the regulation of platelet procoagulant activity. Arch Biochem Biophys. 2009;485:41-48.

17. International Consortium for Blood Pressure Genome-Wide Association Studies, Ehret GB, Munroe PB, Rice KM, Bochud M, Johnson AD, Chasman DI, Smith AV, Tobin MD, Verwoert GC. Genetic variants in novel pathways influence blood pressure and cardiovascular disease risk. Nature. 2011;478:103-109.

18. Levine MA, Eil C, Downs RW, Spiegel AM. Deficient guanine nucleotide regulatory unit activity in cultured fibroblast membranes from patients with pseudohypoparathyroidism type I: a cause of impaired synthesis of 3',5'-cyclic AMP by intact and broken cells. J Clin Invest. 1983;72:316-324.

19. Spiegel AM. Inborn errors of signal transduction: mutations in $G$ proteins and $G$ protein-coupled receptors as a cause of disease. J Inherit Metab Dis. 1997;20:113-121.

20. Ahrens W, Hiort O. Determination of Gsa protein activity in Albright's hereditary osteodystrophy. J Pediatr Endocrinol Metab. 2006;19 Suppl 2:647-651.

21. Levine MA. An update on the clinical and molecular characteristics of pseudohypoparathyroidism. Curr Opin Endocrinol Diabetes Obes. 2012;19:443-451.

22. Patten JL, Johns DR, Valle D, Eil C, Gruppuso PA, Steele G, Smallwood PM, Levine MA. Mutation in the gene encoding the stimulatory $G$ protein of adenylate cyclase in Albright's hereditary osteodystrophy. N Engl J Med. 1990;322:1412-1419.

23. Mantovani G. Pseudohypoparathyroidism: diagnosis and treatment. J Clin Endocrinol Metab. 2011;96:3020-3030.

24. Hayward BE, Barlier A, Korbonits M, Grossman AB, Jacquet P, Enjalbert A, Bonthron DT. Imprinting of the Gsa gene GNAS1 in the pathogenesis of acromegaly. J Clin Invest. 2001;107:R21-R36.

25. Zazo C, Thiele S, Martin C, Fernandez-Rebollo E, Martinez-Indart L, Werner R, Garin I, Spanish PHP Group, Hiort O, Perez de Nanclares G. Gsa activity is reduced in erythrocyte membranes of patients with pseudohypoparathyroidism due to epigenetic alterations at the GNAS locus. J Bone Miner Res. 2011;26:1864-1870.

26. Freson K, Izzi B, Labarque V, Van Helvoirt M, Thys C, Wittevrongel C, Bex M, Bouillon R, Godefroid N, Proesmans W, de Zegher F, Jaeken J, Van Geet C. GNAS defects identified by stimulatory G protein $\alpha$-subunit signalling studies in platelets. J Clin Endocrinol Metab. 2008;93:4851-4859.

27. Freson K, Izzi B, Jaeken J, Van Helvoirt M, Thys C, Wittevrongel C, De Zegher F, Van Geet C. Compound heterozygous mutations in the GNAS gene of a boy with morbid obesity, thyroidstimulating hormone resistance, pseudohypoparathyroidism, and a prothrombotic state. J Clin Endocrinol Metab. 2008;93:4844-4849.

28. Burkhart JM, Vaudel M, Gambaryan S, Radau S, Walter U, Martens L, Geiger J, Sickmann A, Zahedi RP. The first comprehensive and quantitative analysis of human platelet protein composition allows the comparative analysis of structural and functional pathways. Blood. 2012;120:e73-82.

29. Beck F, Geiger J, Gambaryan S, Veit J, Vaudel M, Nollau P, Kohlbacher O, Martens L, Walter U, Sickmann A, Zahedi RP. Time-resolved characterization of CAMP/PKA-dependent signaling reveals that platelet inhibition is a concerted process involving multiple signaling pathways. Blood. 2014;123:e1-e10.

30. Burkhart JM, Gambaryan S, Watson SP, Jurk K, Walter U, Sickmann A, Heemskerk JWM, Zahedi RP. 
What can proteomics tell us about platelets? Circ Res. 2014;114:1204-1219.

31. Siljander P, Farndale RW, Feijge MA, Comfurius P, Kos S, Bevers EM, Heemskerk JW. Platelet adhesion enhances the glycoprotein VI-dependent procoagulant response: involvement of p38 MAP kinase and calpain. Arterioscler Thromb Vasc Biol. 2001;21:618-627.

32. Klaassens M, Blom EW, Schrander JJ, Ris-Stalpers C, Nieuwenhuijzen Kruseman AC, Van Steensel MA, Schrander-Stumpel CT. Unique skin changes in a case of Albright hereditary osteodystrophy caused by a rare GNAS1 mutation. Br J Dermatol. 2010;162:690-694.

33. Gilio K, Van Kruchten R, Braun A, Berna-Erro A, Feijge MAH, Stegner D, Van der Meijden PE, Kuijpers MJE, Varga-Szabo D, Heemskerk JW, Nieswandt B. Roles of STIM1 and Orai1 in glycoprotein VI- and thrombin-dependent procoagulant activity and thrombus formation. J Biol Chem. 2010;285:2362923638.

34. Gilio K, Munnix IC, Mangin P, Cosemans JM, Feijge MA, Van der Meijden PE, Olieslagers S, Chrzanowska-Wodnicka MB, Lillian R, Schoenwaelder S, Koyasu S, Sage SO, Jackson SP, Heemskerk JW. Non-redundant roles of phosphoinositide 3-kinase isoforms alpha and beta in glycoprotein $\mathrm{VI}$ induced platelet signaling and thrombus formation. J Biol Chem. 2009;284:33750-33762.

35. Keularts IM, Van Gorp RM, Feijge MA, Vuist MW, Heemskerk JW. $a_{2 A}$-Adrenergic receptor stimulation potentiates calcium release in platelets by modulating CAMP levels. J Biol Chem. 2000;275:1763-1772.

36. Van Kruchten R, Cosemans JM, Heemskerk JW. Measurement of whole blood thrombus formation using parallel-plate flow chambers: a practical guide. Platelets. 2012;23:229-242.

37. De Witt SM, Swieringa F, Cavill R, Lamers MM, Van Kruchten R, Mastenbroek T, Baaten C, Coort S, Pugh N, Schulz A, Scharrer I, Jurk K, Zieger B, Clemetson KJ, Farndale RW, Heemskerk JW, Cosemans JM. Identification of platelet function defects by multi-parameter assessment of thrombus formation. Nat Commun. 2014;5:4257.

38. Swieringa F, Kuijpers MJ, Lamers MM, Van der Meijden PE, Heemskerk JW. Rate-limiting roles of tenase complex of factors VIII and IX in platelet procoagulant activity and formation of plateletfibrin thrombi under flow. Haematologica. 2015;100:748-756.

39. Engholm-Keller K, Birck P, Storling J, Pociot F, Mandrup-Poulsen T, Larsen MR. TiSH: a robust and sensitive global phosphoproteomics strategy employing a combination of $\mathrm{TiO}_{2}$, SIMAC, and HILIC. J Proteomics. 2012;75:5749-5761.

40. Dickhut C, Radau S, Zahedi RP. Fast, efficient, and quality-controlled phosphopeptide enrichment from minute sample amounts using titanium dioxide. Methods Mol Biol. 2014;1156:417-430.

41. Larsen MR, Graham ME, Robinson PJ, Roepstorff P. Improved detection of hydrophilic phosphopeptides using graphite powder microcolumns and mass spectrometry: evidence for in vivo doubly phosphorylated dynamin I and dynamin III. Mol Cell Proteomics. 2004;3:456-465.

42. Thingholm TE, Palmisano G, Kjeldsen F, Larsen MR. Undesirable charge-enhancement of isobaric tagged phosphopeptides leads to reduced identification efficiency. J Proteome Res. 2010;9:40454052.

43. Taus T, Kocher T, Pichler P, Paschke C, Schmidt A, Henrich C, Mechtler K. Universal and confident phosphorylation site localization using phosphoRS. J Proteome Res. 2011;10:5354-5362.

44. Xue Y, Ren J, Gao X, Jin C, Wen L, Yao X. GPS 2.0, a tool to predict kinase-specific phosphorylation sites in hierarchy. Mol Cell Proteomics. 2008;7:1598-1608.

45. Schwarz UR, Geiger J, Walter U, Eigenthaler M. Flow cytometry analysis of intracellular VASP phosphorylation for the assessment of activating and inhibitory signal transduction pathways in human platelets: definition and detection of ticlopidine/clopidogrel effects. Thromb Haemost. 1999;82:1145-1152.

46. GeneCards. www.genecards.org. 2015

47. Subramanian H, Zahedi RP, Sickmann A, Walter U, Gambaryan S. Phosphorylation of CalDAG-GEFI by protein kinase A prevents Rap1b activation. J Thromb Haemost. 2013;11:1574-1582. 


\section{Supplemental data}
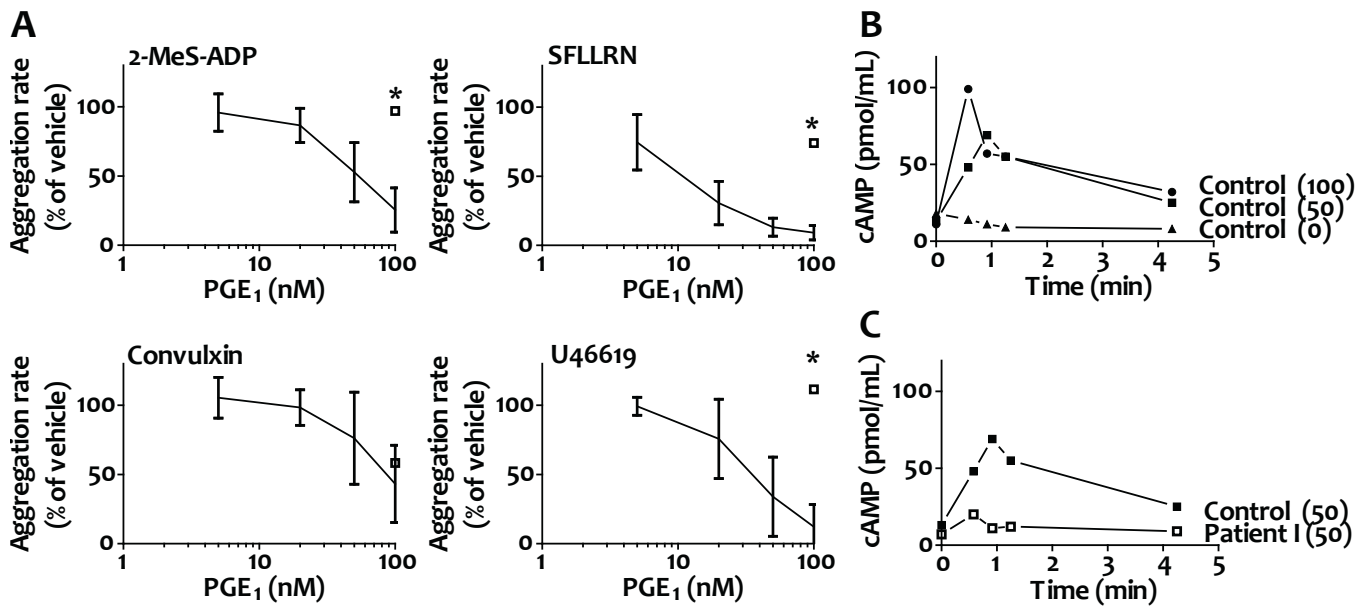

Supplemental Figure 1. Impaired $\mathrm{PGE}_{1}$-induced inhibition of aggregation and $\mathrm{CAMP}^{-}$elevation in platelets from AHO patient. Platelets in PRP were pretreated with vehicle or indicated concentration of $\mathrm{PGE}_{1}$ for 4 minutes. (A) Effect of $\mathrm{PGE}_{1}$ on platelet aggregation rate after stimulation with 2-MeS-ADP $(5 \mu \mathrm{M})$, SFLLRN $(15 \mu \mathrm{M})$, convulxin $(10 \mathrm{ng} / \mathrm{mL})$ or U46619 $(5 \mu \mathrm{M})$, as indicated. Data are from 10 healthy control subjects and patient I (open squares). (B, C) Effect of $P G E_{1}$ treatment $(0,50,100 \mathrm{nM})$ on platelet CAMP level in time. Dose-dependency of $\mathrm{PGE}_{1}$ effect on platelets from a control subject (B); and comparison between platelets from control subject (day control) and patient I (C). Means \pm SD ( $n=10$ controls); $p$ $<0.05$.
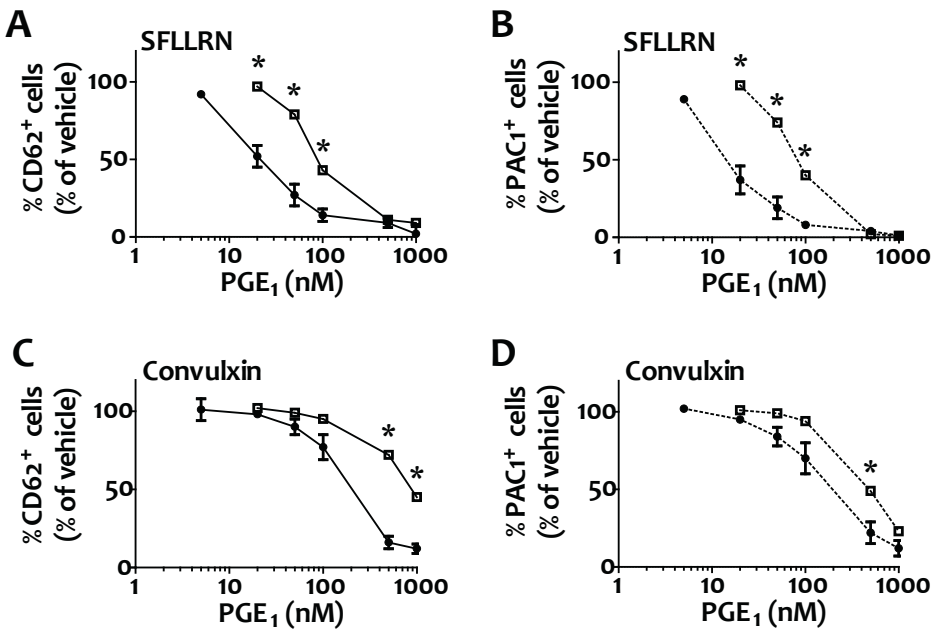

\footnotetext{
Supplemental Figure 2. Impaired $\mathrm{PGE}_{1}$-induced inhibition of integrin activation and secretion in platelets from AHO patient. Platelets in PRP were treated with vehicle or $\mathrm{PGE}_{1}$ for 4 minutes and activated with SFLLRN (15 $\mu \mathrm{M}$; A, B) or convulxin (10 ng/mL; C, D). Extent of secretion (\% of CD62P-positive platelets) and integrin $\alpha_{\| \mathrm{lb}} \beta_{3}$ activation (\% of PAC1-positive platelets) were determined with FITC-labelled antiCD62P and PAC1 mAb, respectively. Data are from 6 healthy control subjects (closed dots) and patient I (open squares), and are expressed as percentages of the vehicle condition. Means $\pm \operatorname{SD}$ ( $n=6$ controls); ${ }^{*} p<0.05$.
} 

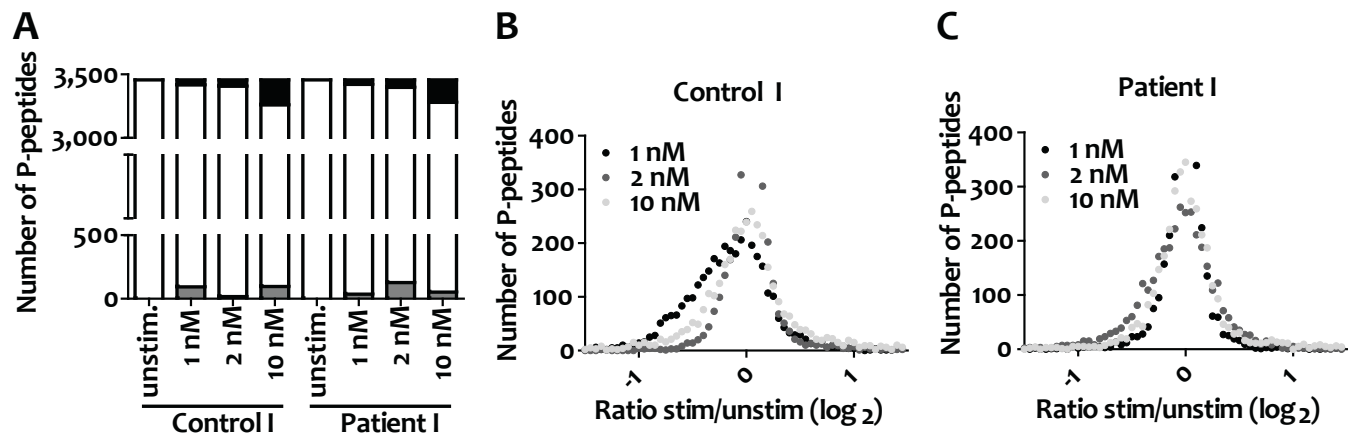

Supplemental Figure 3. Alterations in iloprost-induced phosphoproteome of platelets from a patient with AHO. Phospho-proteomics analysis using iTRAQ labelling (4 patient samples, 4 control samples) gave ratioed information on 3,457 phospho-peptides, corresponding to 1,356 unique proteins, for the conditions: unstimulated and 1, 2 or $10 \mathrm{nM}$ iloprost. Ratio values per peptide were log2 transformed. Ratios outside the range of median (0.00) \pm 2 SD (SD: 0.385) based on log2 values were considered as relevant differences in phosphorylation. (A) Numbers of phospho-peptides per condition considered to be increased (black) or decreased (grey) by iloprost simulation. (B, C) Histograms representing ratios per phospho-peptide of the effects of 1, 2 or $10 \mathrm{nM}$ iloprost for samples from control I (B) and patient I (C). 
Supplemental Table 1. Ranked list of top 95 platelet proteins with increased iloprost-induced phosphorylation in control platelets and changes in AHO platelets. Color codes: green = ratio increased in in response to iloprost; red = ratio lower in patient vs. control; yellow = ratio higher in patient vs. control; blue $=$ previously identified iloprost-induced phosphorylation or positive PKA score. Changes were considered relevant when outside the range of median $\pm 2 \times 0.385$ (log2 transformed). Abbreviations: n.d., not detected; n.s., not significant.

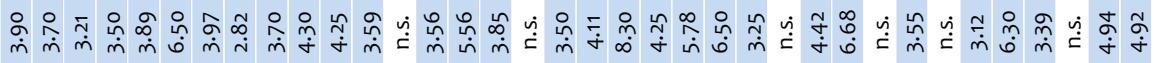

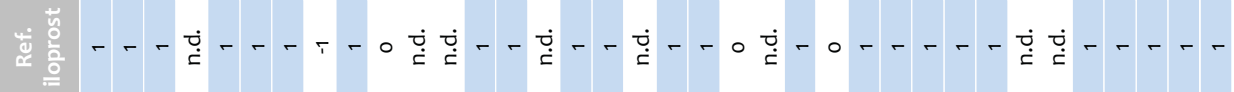

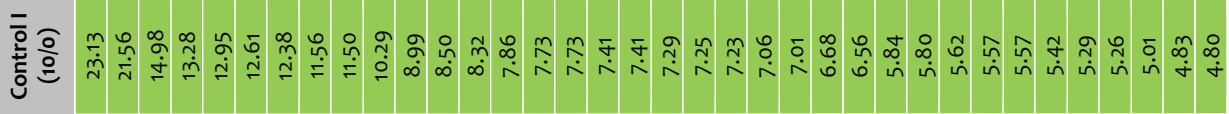

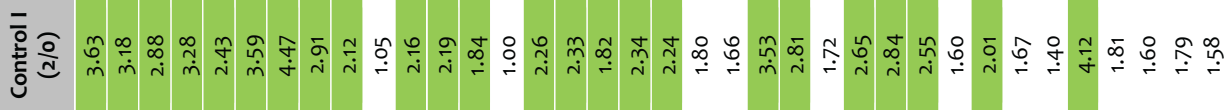

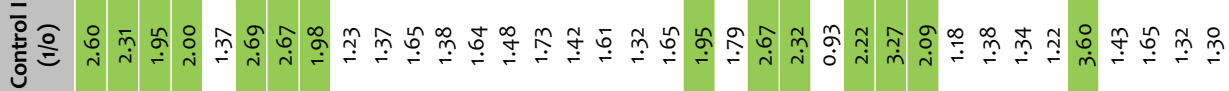

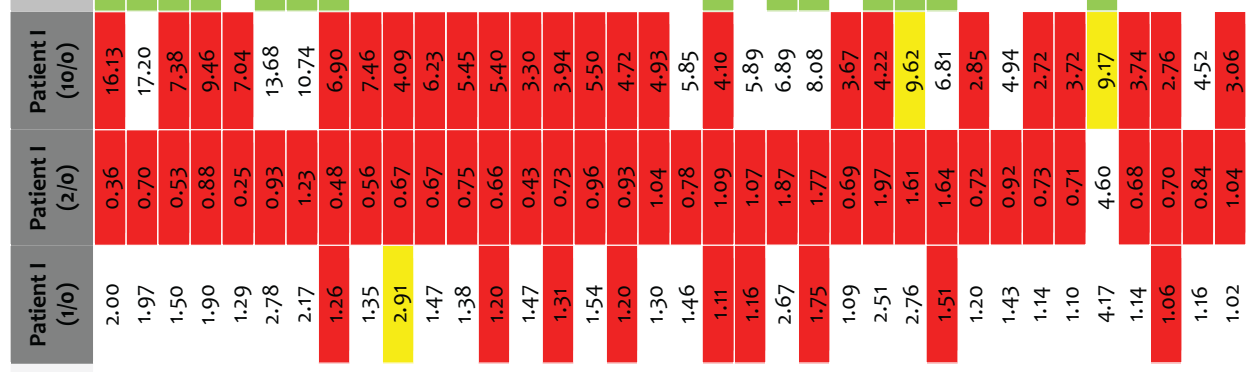

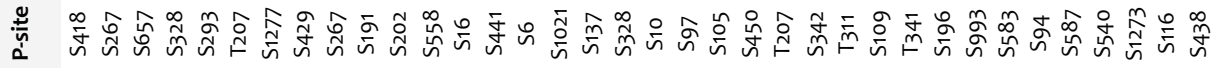

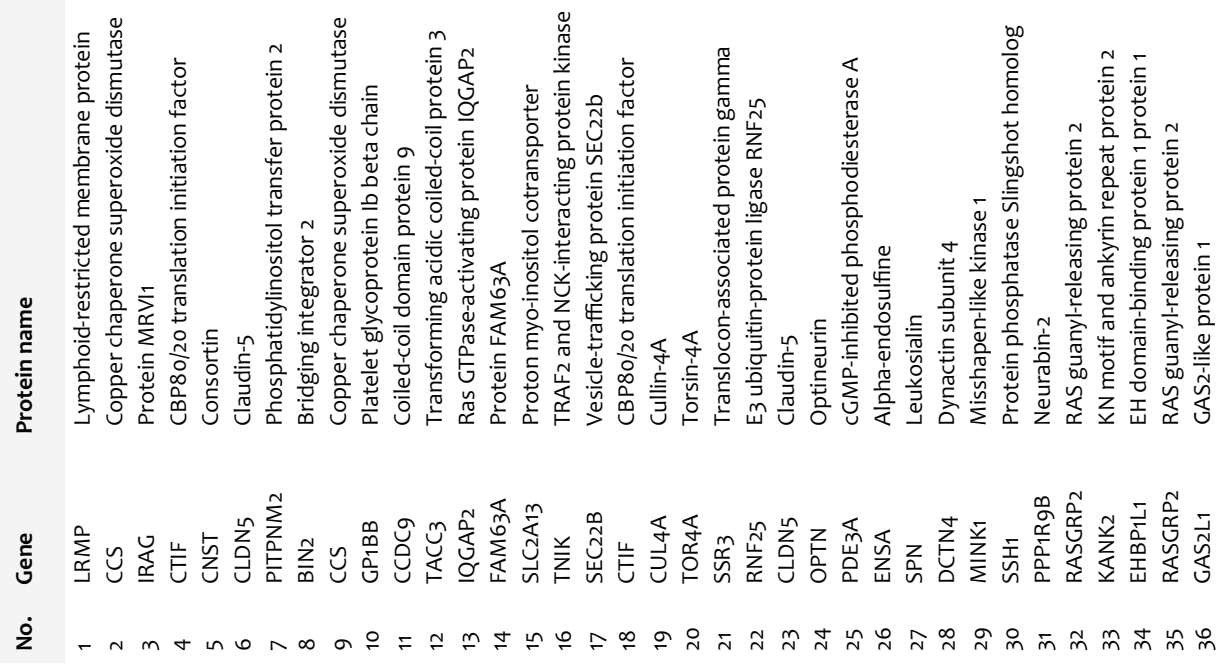




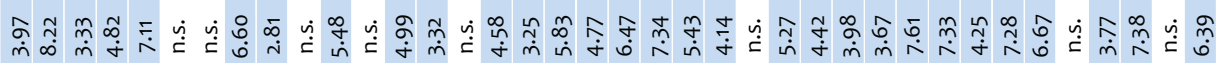

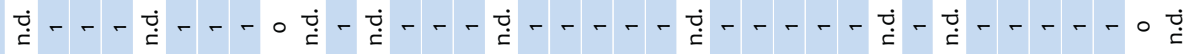

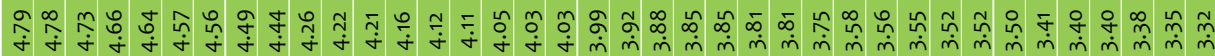

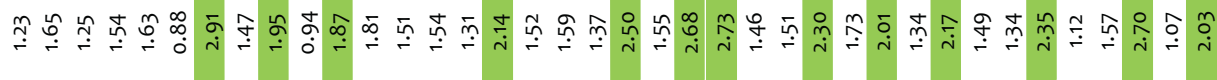

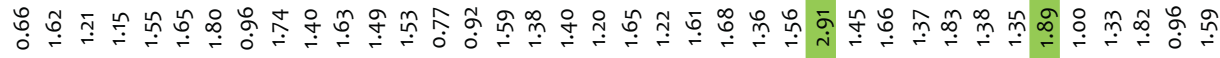

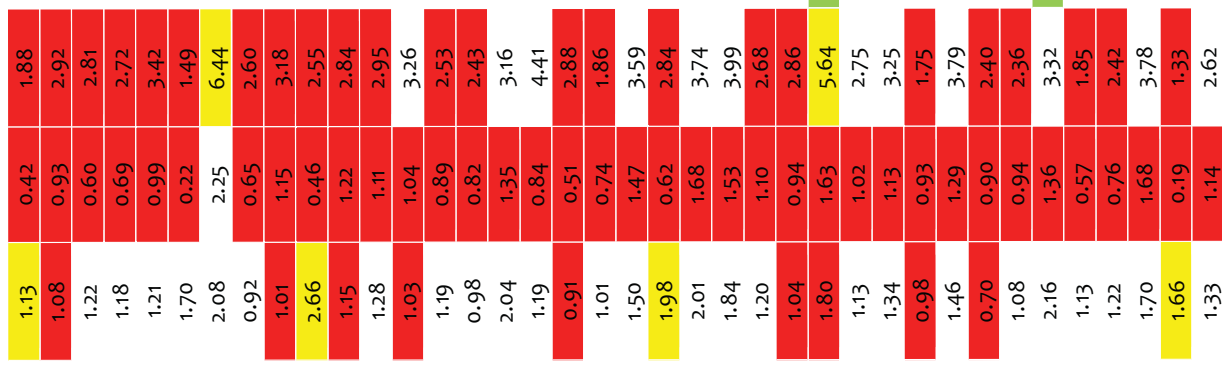

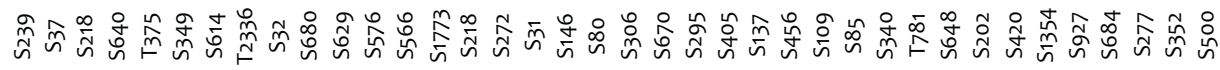

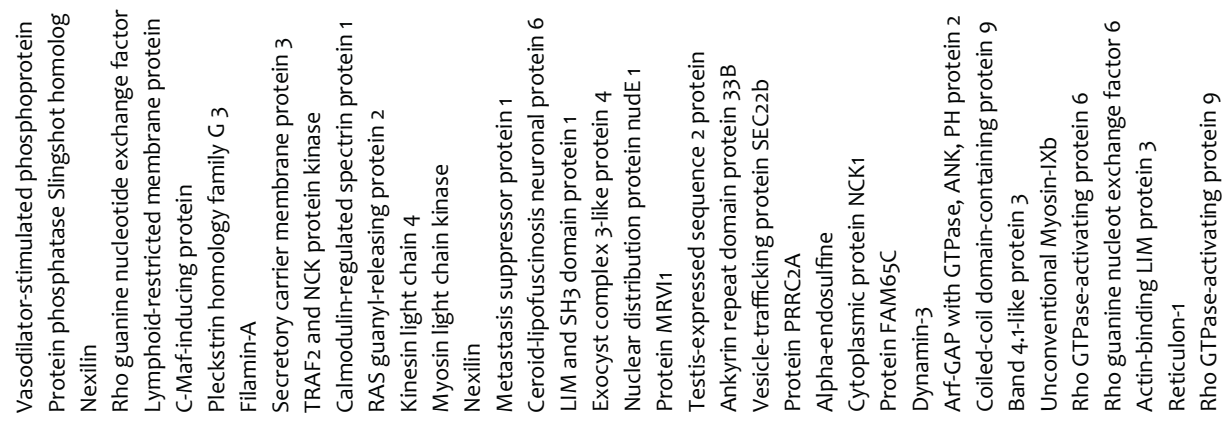

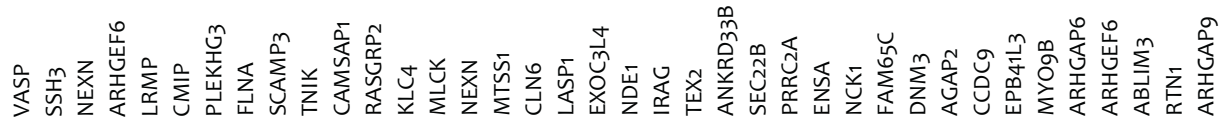

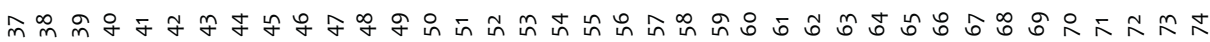




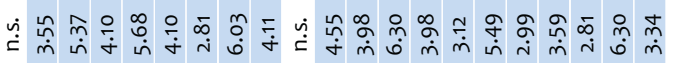

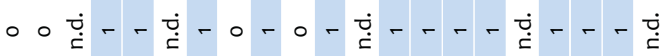

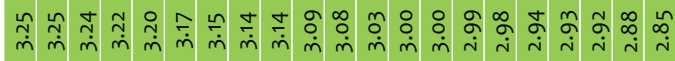

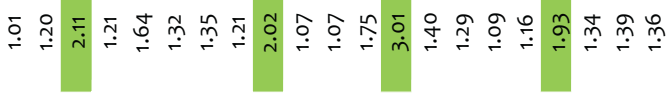

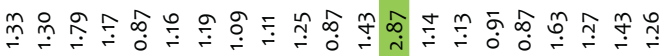

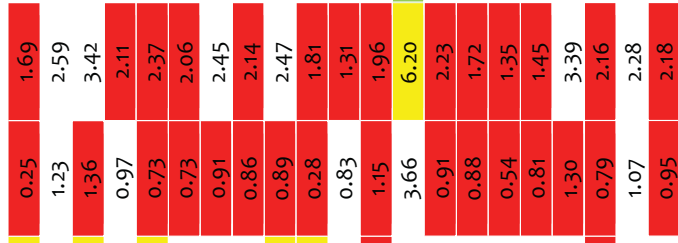

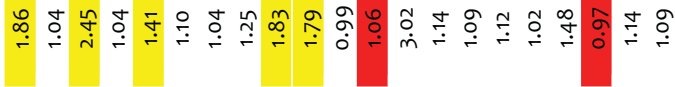

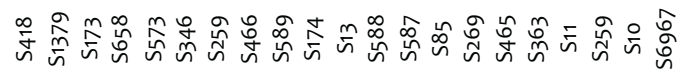

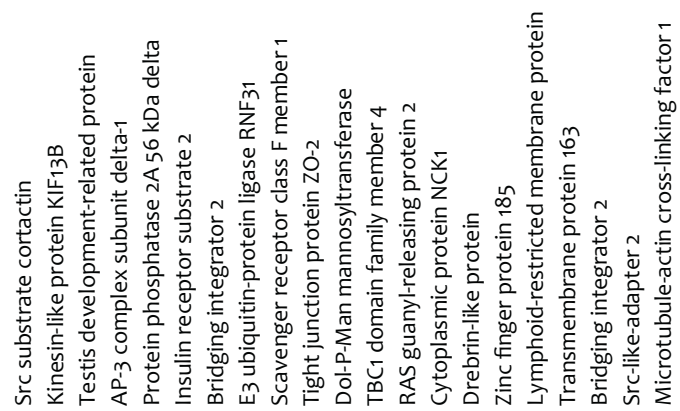

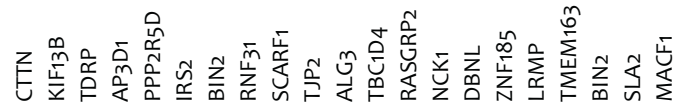

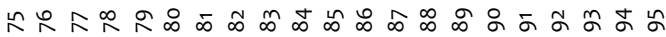





\section{CHAPTER 4}

Desmopressin treatment improves platelet function under flow in patients with postoperative bleeding

Swieringa F, Lancé MD, Fuchs B, Feijge $M A$, Solecka BA, Verheijen LP, Hughes KR, Van Oerle R, Deckmyn H, Kannicht C, Heemskerk JW, Van der Meijden PE

J Thromb Haemost. 2015;13:1503-13 Reprinted with permission 


\begin{abstract}
Patients undergoing major cardiothoracic surgery are subjected to dilution, owing to massive fluid infusion and blood component transfusion. These patients may experience bleeding perioperatively, and are frequently treated with the endotheliumactivating agent desmopressin. Our aim was to investigate the effect of desmopressin administration on von Willebrand factor (VWF)-dependent coagulant and platelet functions under flow conditions. Thereto, blood from 16 patients with postoperative bleeding was obtained before and after desmopressin treatment $(0.3 \mu \mathrm{g} / \mathrm{kg}$ body weight), and assessed for coagulant properties and platelet function. Furthermore, VWF antigen levels and multimer composition were determined in both samples. We found that desmopressin treatment did not change thrombin generation in plasma or whole blood thromboelasticity. Also coagulation factor levels (other than factor VIII) and coagulation times were unchanged, suggesting that desmopressin treatment did not have a major effect on the coagulant activity. On the other hand, desmopressin treatment raised the already high plasma levels of VWF from a median of $116 \mathrm{IU} / \mathrm{mL}$ (interquartile range [IQR] 102-154 IU/mL) to a median of $160 \mathrm{IU} / \mathrm{mL}(\mathrm{IQR} 126-187 \mathrm{IU} /$ $\mathrm{mL})(p=0.007)$, owing to accumulation of the high molecular weight VWF multimers. Furthermore, desmopressin treatment caused an increase in collagen-dependent thrombus formation and platelet phosphatidylserine exposure. Markers of thrombus formation correlated with the plasma levels of VWF. In vitro control experiments confirmed a major contribution of VWF to thrombus formation and procoagulant activity under conditions of blood dilution. Thus, desmopressin treatment of patients with bleeding complications after cardiothoracic surgery induces the release of high molecular weight VWF multimers, which enhance platelet activation and thrombus formation under flow conditions.
\end{abstract}

\title{
Introduction
}

Haemostasis is a complex process, in which blood platelets participate to form a primary plug, and von Willebrand factor (VWF) acts as an important plasma component carrying factor VIII and enhancing platelet adhesion to the damaged vessel wall in flowing blood. ${ }^{1-3}$ Adhered platelets and VWF support the processes of thrombin and fibrin generation, which are required for a stable platelet-fibrin clot. ${ }^{4-6}$

The plasma VWF is synthesised in endothelial cells and megakaryocytes as a dimeric protein, which multimerises in the post-Golgi compartments. ${ }^{7}$ In endothelial cells, it is, in part, constitutively released into the blood plasma, and in part stored in the Weibel-Palade bodies, from which it can be liberated upon endothelial activation. ${ }^{8}$, ${ }^{9}$ Characteristically, the stored pool of VWF contains ultralarge multimers $(>10,000$ $\mathrm{kDa}$, where one dimer is equivalent to $500 \mathrm{kDa}) .^{10}$ Once secreted into the blood plasma, these ultralarge multimers are highly active in capturing platelets under flow conditions. ${ }^{11-13}$ However, they are gradually cleaved by the protease ADAMTS13, leading 
to the accumulation of high, intermediate and low molecular weight multimers (500$10,000 \mathrm{kDa}$ ), the last of which are unable to bind platelets. ${ }^{14,15}$ On the other hand, multimers of all lengths retain their function as carriers for FVIII. ${ }^{10}$

Desmopressin (1-deamino-8-D-arginine vasopressin or DDAVP), a synthetic analogue of the antidiuretic hormone vasopressin, is used clinically for patients with a bleeding risk resulting from mild von Willebrand disease or haemophilia A, in order to stimulate degranulation of the Weibel-Palade bodies via type 2 vasopressin receptors, and to increase the plasma level of VWF. ${ }^{16,17}$ Desmopressin is also a treatment option for patients who have been subjected to major dilution after surgery and later suffer from massive bleeding during recovery in the intensive care unit. ${ }^{16}$ Patients undergoing cardiothoracic operations with extensive surgical trauma and blood dilution are at special risk for this postsurgery bleeding..$^{18-20}$ Several studies have shown that desmopressin treatment of these patients can restore haemostasis, ${ }^{21,22}$ although other studies have obtained less favourable results. ${ }^{23,24}$ This is considered to be a consequence of acute VWF release and improved VWF-dependent haemostatic activity, but this has not been examined in detail. Here, we investigated the effect of desmopressin treatment on VWF-dependent coagulant and platelet functions under flow conditions. We report a platelet-activating effect related to the appearance of high molecular weight VWF multimers, even in patients with already high VWF levels prior to treatment.

\section{Materials and methods}

\section{Patients and blood sampling}

Experiments were approved by the local Medical Ethics Committee (MEC 12-4-096). Blood samples were taken from 16 patients who had undergone cardiothoracic surgery with extracorporeal circulation and experienced postoperative bleeding. During surgery the patients had undergone dilution by infusion with crystalloids and cardiopulmonary bypass priming fluid with a total volume of 4.5-5.5 L (see Table 1). All 16 patients received tranexamic acid and heparin as part of standard transfusion management. Several patients needed to be transfused with packed red cells (5/16), platelet concentrate (6/16), fresh frozen plasma (4/16), and/or fibrinogen concentrate $(1 / 16)$. After surgery, heparin was fully antagonised with protamine in all patients; this was checked from coagulation times.

All patients experienced bleeding while recovering in the intensive care unit. Transfusion treatment was required with tranexamic acid (9/16), hydroxyethyl starch (6/16), packed red cells (13/16), platelet concentrate (8/16), fresh frozen plasma (5/16). Total volume of fluid infusion was 1410 (670-2580) $\mathrm{mL}$ (median, interquartile range). Median blood drainage postoperatively was $1260 \mathrm{~mL}$ per 24 hours.

Desmopressin $(0.3 \mu \mathrm{g} / \mathrm{kg}$ body weight) was given on the basis of the clinical observation of persistent blood loss exceeding $200 \mathrm{~mL} / \mathrm{h}$. Desmopressin treatment 
was given at a median of 70 minutes (interquartile range [IQR] 37-94 minutes) after the end of surgery. Blood samples were taken immediately before and at 1 hour after desmopressin administration. As last resort to stop bleeding, one patient received fibrinogen concentrate ( $1 \mathrm{~g}$ ) after desmopressin. In this patient, the plasma fibrinogen level increased from 1.7 to $2.2 \mathrm{~g} / \mathrm{L}$.

Blood samples were collected into $10.5 \mathrm{mM}$ trisodium citrate to assess thromboelastometry, coagulant activities, thrombus formation, and VWF multimer distribution. Blood was also obtained from healthy volunteers who were free from antiplatelet or anticoagulant medication for at least 2 weeks, after informed consent had been obtained in accordance with the Declaration of Helsinki. Blood samples for isolation of washed platelets were collected into acid-citrate-dextrose (ACD: $13.3 \mathrm{mM}$ trisodium citrate, $8.7 \mathrm{mM}$ citric acid and $30 \mathrm{mM}$ glucose). Where indicated, citrated whole blood samples were diluted with saline medium, consisting of $137 \mathrm{mM} \mathrm{NaCl}$, $12.9 \mathrm{mM}$ trisodium citrate, $2 \mathrm{mM} \mathrm{CaCl}_{2}$, and $2 \mathrm{mM} \mathrm{MgCl}$, in order to maintain equal levels of free $\mathrm{Ca}^{2+}$ and $\mathrm{Mg}^{2+}$ upon dilution.

\section{Preparation of platelets and plasma}

Washed platelets were prepared from ACD-anticoagulated blood of healthy donors as previously described, ${ }^{25}$ and resuspended in Hepes buffer $\mathrm{pH} 7.45$ (10 mM Hepes, 136 $\mathrm{mM} \mathrm{NaCl}, 2.7 \mathrm{mM} \mathrm{KCl}, 2 \mathrm{mM} \mathrm{MgCl}, 0.1 \%$ glucose (w/v) and $0.1 \%$ bovine serum albumin $(\mathrm{w} / \mathrm{v})$ ). Platelet count was determined with a thrombocounter (Coulter Electronics, Luton, UK). Platelet-free plasma was prepared from citrate-anticoagulated blood that was centrifuged twice at 2,630 $\times$ g for 10 minutes..$^{25}$ The collected plasma was immediately snap-frozen in liquid nitrogen, and was stored at $-80^{\circ} \mathrm{C}$ until use.

\section{Plasma coagulation analysis}

The activated partial thrombin time (APTT) was determined with standard in-hospital methods. ${ }^{20}$ Antigen levels of VWF were determined by nephelometry. ${ }^{26}$ Plasma levels of fibrinogen were measured according to the Clauss method; activities of prothrombin and FVIII were determined by dilution in factor-deficient plasmas, and with one-stage clotting tests. ${ }^{20}$

\section{Thrombin generation}

Thrombin generation in plasma was measured with the calibrated automated thrombogram method. ${ }^{6,} 27$ Plasma samples were supplemented with $4 \mu \mathrm{M}$ phospholipid vesicles or with platelets from a single healthy donor $\left(100 \times 10^{9} / \mathrm{L}\right)$, as desired. Phospholipid vesicles contained phosphatidylserine, phosphatidylcholine and phosphatidylethanolamine, prepared in a 1:3:1 ratio (Avanti Polar Lipids, Alabaster AL, USA). Assays were performed in triplicate at $37{ }^{\circ} \mathrm{C}$ in 96 -well round- 
bottom plates (Thermo Fischer Scientific, Milford MA, USA) in the presence of 2.5 $\mathrm{mM}$ thrombin substrate Z-Gly-Gly-Arg aminomethyl coumarin (Z-GGR-AMC; Bachem, Bubendorf, Switzerland). Triggering was performed with $10 \mathrm{pM}$ tissue factor and 16.7 $\mathrm{mM} \mathrm{CaCl}$ (Innovin; Dade Behring, Deerfield IL, USA), as previously optimised with patient plasmas. ${ }^{28}$ Fluorescence accumulation from cleaved aminomethyl coumarin was measured over time, and first-derivative curves were converted into nanomolar concentrations of thrombin with Thrombogram software. ${ }^{25}$ Maximal levels of thrombin generated (thrombin peak height) and areas under the curve (endogenous thrombin potential [ETP]) were derived from the thrombin generation curves.

\section{Thromboelastometry}

Elastic fibrin clot formation was assessed with citrate-anticoagulated whole blood by rotational thromboelastometry (TEM International, Munich, Germany). As previously described, ${ }^{29}$ blood samples were triggered in triplicate with $10 \mathrm{pM}$ tissue factor and $16.7 \mathrm{mM} \mathrm{CaCl}_{2}$. Curves were analysed for time to onset of clot formation, clot formation time, maximum clot firmness, and slope of elastic clot formation.

\section{Platelet activation and thrombus formation under flow}

Thrombus formation under flow conditions was determined by perfusion of citrated whole blood over a collagen-coated glass coverslip in a transparent parallel-plate perfusion chamber (width $3 \mathrm{~mm}$, depth $50 \mu \mathrm{m}$ ). ${ }^{30}$ Coverslips were coated with microspots of $0.5 \mu \mathrm{L}$ of Horm type I collagen $(100 \mu \mathrm{g} / \mathrm{mL}$; Nycomed Pharma, Munich, Germany). Coated coverslips were blocked with $1 \%$ bovine serum albumin, and rinsed with Hepes buffer supplemented with $2 \mathrm{mM} \mathrm{CaCl}_{2}$ and $1 \mathrm{U} / \mathrm{mL}$ heparin. Prior to perfusion, samples of $400 \mu \mathrm{L}$ of blood were supplemented with $40 \mu \mathrm{M} \mathrm{D}$-phenylalanylL-prolyl-L-arginine chloromethyl ketone (PPACK; Santa Cruz, Heidelberg, Germany) and $40 \mathrm{U} / \mathrm{mL}$ fragmin (Dalteparine; Pfizer, Capelle aan de IJssel, The Netherlands). During perfusion, these samples were recalcified with Hepes buffer $(\mathrm{pH} 7.45)$ containing $7.5 \mathrm{mM} \mathrm{CaCl}_{2}$ and $3.75 \mathrm{mM} \mathrm{MgCl}_{2}$ to achieve physiologic (millimolar) levels of $\mathrm{Ca}^{2+}$ and $\mathrm{Mg}^{2+} .{ }^{31}$ Blood perfusion was for 4 minutes at a wall shear rate of 1,000 $\mathrm{s}^{-1}$. Thrombi were poststained for exposure of phosphatidylserine with $0.5 \mu \mathrm{g} / \mathrm{mL}$ annexin A5 labelled with fluorescein isothiocyanate (FITC) (PharmaTarget, Maastricht, The Netherlands) or Alexa Fluor-647 (Invitrogen, Bleiswijk, The Netherlands), or for P-selectin expression with FITC-labelled a-CD62P mAb (Immunotech, Marseille, France) in rinse buffer. Where indicated, blood samples were pretreated with the membrane dye $\operatorname{DiOC}_{6}$ (Anaspec, Reeuwijk, The Netherlands), ${ }^{32}$ and with the VWFneutralising mAb 82D6A3 $(20 \mu \mathrm{g} / \mathrm{mL})^{33,34}$ and/or VWF concentrate (150 IU/mL; Wilate; Octapharma Pharmazeutika, Vienna, Austria) for 15 minutes at $37^{\circ} \mathrm{C}$.

Phase-contrast and fluorescence images were taken with a microscopic imaging system (Visitech, Sunderland, UK) ${ }^{35}$ or an LSM7 Live inverted confocal fluorescence 
microscope (Zeiss, Oberkochen, Germany). ${ }^{36}$ Images were analysed for surface area coverage with imageJ (open source) and Metamorph offline software (Molecular Devices, Sterling Heights MI, USA), as previously described. ${ }^{36}$

\section{VWF multimer analysis}

Samples of platelet-free plasma from patients were analysed for VWF multimer composition with horizontal discontinuous gel electrophoresis equipment (Multiphor II; GE Healthcare Biosciences, Uppsala, Sweden) and $1.2 \%$ agarose gels, as previously described. ${ }^{14}$ The frozen patient plasmas were prethawed at $37{ }^{\circ} \mathrm{C}$ under rotation to prevent protein loss from cryoprecipitation. ${ }^{37}$ Defrosted samples were diluted with sample buffer (10 mM Tris, $1 \mathrm{mM} \mathrm{Na} \mathrm{F}_{2}$ EDTA, $15 \%[\mathrm{v} / \mathrm{v}]$ glycerin, $2 \%[\mathrm{w} / \mathrm{w}] \mathrm{SDS}$, and bromophenol blue, $\mathrm{pH}$ 8.0) to a final concentration of $0.05 \mathrm{IU} / \mathrm{mL} \mathrm{VWF}$ antigen. Standard human control plasma (Haemochrom Diagnostica, Essen, Germany) was diluted to the same concentration. After gel electrophoresis for 16-18 hours, proteins were electroblotted onto nitrocellulose membranes with a Hoefer tank blot unit. Blotted membranes were blocked with $1.5 \%$ ultrahigh-temperature processed milk, and incubated overnight with 1:2,000 horseradish peroxidase-conjugated polyclonal rabbit anti-VWF antibody (Biozol Diagnostica, Eching, Germany). After immunostaining, the membranes were washed twice with $1.5 \%$ milk and extensively rinsed with water, and the chemiluminescence signal was visualised with a SuperSignal West Pico substrate (Pierce, Rockford NY, USA) in a LAS-1000 image analyser (Fujifilm, Tokyo, Japan). Densitometric analysis of blots was performed with Multigauge V3.2 software (Fujifilm).

\section{Statistical analysis}

Patient-related data are presented as medians with IQRs. Treatment effects of desmopressin were tested with a Wilcoxon matched-pairs signed rank test. Associations of parameters were tested by Pearson (partial) correlation analysis; VWF multimer distributions were compared by use of a chi-squared test. SPSS 18.0 software was used (IBM, Amsterdam, The Netherlands). P-values of $<0.05$ were considered to be statistically significant.

\section{Results}

Patient characteristics and effects of desmopressin treatment on plasma parameters

The prohaemostatic effects of desmopressin treatment were determined in 16 patients who had undergone cardiothoracic surgery and experienced postoperative bleeding. Of these patients, 10 had a coronary artery bypass graft, three had an aortic valve replacement because of severe aortic stenosis, one had a mitral valve 
Table 1. Patient and surgery characteristics and haematological parameters, of surgery patients $(n=16)$ treated with desmopressin during recovery. APTT, activated partial thrombin time; IQR, interquartile range. Values are from blood samples taken before and 1 hour after treatment $* * p<0.01$ versus before treatment. Normal presurgery values for this patient group $(n=48, \# n=7)$ were as reported previously. ${ }^{29}$

\section{Patient characteristics}

$\begin{array}{ll}\text { Male/female (n) } & 12 / 4 \\ \text { Age (years; median [IQR]) } & 63(57-72) \\ \text { Weight (kg; median [IQR]) } & 79(75-91) \\ \text { Antiplatelet medication (n) } & \text { Aspirin (14), } \\ & \text { Clopidogrel (2) }\end{array}$

\section{Surgery characteristics}

Coronary artery bypass graft $(n)$

Valve replacement $(n)$

Open stent graft (n)

Re-thoracotomy $(n)$

Pump duration (min; median [IQR])

Total fluid infused $(\mathrm{L})$

Transfusion components ( $\mathrm{n}$ )

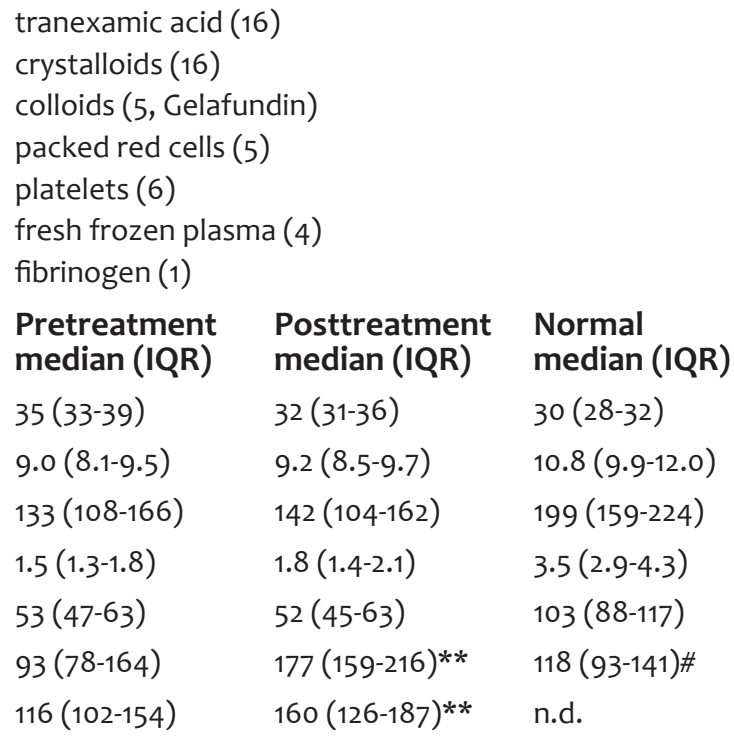

\section{Haematological parameters}

aPTT $(\mathrm{s})$
Haemoglobin $(\mathrm{g} / \mathrm{dL})$
Platelet count $\left(\times 10^{9} / \mathrm{L}\right)$
Fibrinogen $(\mathrm{g} / \mathrm{L})$
Prothrombin $(\%)$
Factor VIII $(\%)$
VWF antigen (IU/mL)

replacement because of mitral regurgitation, one had open stent grafting with an endoprosthesis for a thoracoabdominal aortic aneurysm, and one had a rethoracotomy. All patients underwent major dilution during the invasive surgery (4.5-5.5 L), in several cases requiring blood component transfusion (Table 1). While recovering in the intensive care unit, the patients experienced major blood loss, which was treated by transfusion with tranexamic acid, hydroxyethyl starch, packed red cells, platelet concentrate, and/or fresh frozen plasma. The total volume of fluid 
infused was $1410 \mathrm{~mL}$ (IQR 670-2,580 mL) (see Methods). Owing to persistent blood loss, the patients were treated with desmopressin ( $0.3 \mu \mathrm{g} / \mathrm{kg})$.

As a last resort to stop bleeding, one patient also received fibrinogen (1 $\mathrm{g}$ ) during the time of desmopressin treatment. In 14 of 16 patients, bleeding stopped within 1 hour. Two other patients required rethoracotomy because of ongoing blood loss, for surgical reasons. Blood samples were taken from all patients immediately before and at 1 hour after desmopressin treatment.

Measurement of haemostatic parameters confirmed plasma dilution during surgery before treatment with desmopressin (Table 1 ). The median platelet count was $133 \times 10^{9} / \mathrm{L}\left(\right.$ IQR $\left.108-166 \times 10^{9} / \mathrm{L}\right)$, the haemoglobin level was $9.0 \mathrm{~g} / \mathrm{dL}(\mathrm{IQR} 8.1-9.5 \mathrm{~g} / \mathrm{dL})$, the fibrinogen level was $1.5 \mathrm{~g} / \mathrm{L}$ (IQR 1.3-1.8 g/L), the prothrombin level was $53 \%$ (IQR $47-63 \%$ ) of normal, and the APTT was 35 seconds (IQR 3339 seconds). In agreement with previous observations in other patients subjected to the same type of surgery, ${ }^{29}$, ${ }^{38}$ levels of FVIII were less decreased, i.e. $93 \%$ (IQR 78-164\%) of that in normal plasma, whereas VWF levels were increased to $116 \%$ (IQR 102-154\%) of that in normal plasma.

After infusion of desmopressin, no significant differences were observed for the patient group in blood cell counts (i.e. haemoglobin levels and platelet counts), fibrinogen and prothrombin levels, or coagulation times (Table 1). The unchanged level of prothrombin indicated that, overall, the extent of dilution in the patient group remained similar..$^{20}$ In marked contrast, posttreatment levels of FVIII and VWF further increased to $177 \%$ (IQR 159-216\%) and 160\% (IQR 126-187\%), respectively, after desmopressin treatment (Table 1). When examined per patient, changes in blood cell count did not relate to transfusion of red cells or platelets, most likely because of additional blood loss.

Lack of effect of desmopressin treatment on thrombin generation and fibrin clot formation

In view of the earlier postulated role of VWF in supporting the coagulation process, ${ }^{5,39}$ we used patient plasmas before and after desmopressin treatment for determination of thrombin generation. Assays were carried out with plasma samples supplemented with either procoagulant phospholipids or donor platelets, which were activated with tissue factor $/ \mathrm{CaCl}_{2}$ according to protocols optimised previously. ${ }^{40}$ In the presence of phospholipids, the main pretreatment parameters of thrombin peak height and ETP (area under the curve) were approximately 50-70\% of normal values (Table 2). This corresponded to a similar reduction in prothrombin level, and confirmed the dilutional state of the patients. With platelets present, both pretreatment parameters were $85-90 \%$ of normal values, thus confirming the earlier conclusion that platelets partly compensate for the effect of dilution on thrombin generation. ${ }^{28,29}$ However, no significant differences were seen in thrombin generation parameters of patient plasmas taken before or after desmopressin treatment, regardless of whether platelets or 
Table 2. Thrombin generation in plasma from patients before and after desmopressin treatment. ETP, endogenous thrombin potential; IQR, interquartile range. Plasma samples were supplemented with donor platelets $\left(100 \times 10^{9} / \mathrm{L}\right)$ or phospholipid vesicles $(4 \mu \mathrm{M})$, and activated with tissue factor $(10$ pM). Thrombin generation was measured with a fluorescent substrate in the calibrated automated thrombogram assay. Shown are values of thrombin peak height and ETP (area-under-the-curve). Number of patients $=13, p>0.10$ for all samples versus pretreatment. Normal values presurgery of this patient group $(n=48)$ in the presence of phospholipids or platelets are as follows: for peak height, $297 \mathrm{nM}$ (IQR 252-325 nM) and $145 \mathrm{nM}$ (IQR 115-166 nM), respectively; and for ETP, 1,159 $\mathrm{nM} \times \min (\mathrm{IQR} 1,001-1,304 \mathrm{nM} \times \min )$ and $1,282 \mathrm{nM} \times \min (\mathrm{IQR} 1,108-1,411 \mathrm{nM} \times \min )$, respectively. ${ }^{29}$

\section{Condition}

Phospholipids pretreatment $177(164-190)$

posttreatment $172(152-198)$

Platelets pretreatment $130(128-130)$

posttreatment $123(113-145)$

\section{$\operatorname{ETP}(\mathrm{nM} \times \mathrm{min})$ median (IQR)}

$830(673-905)$

$797(695-910)$

$1,072(972-1,172)$

$1,071(988-1,138)$
A

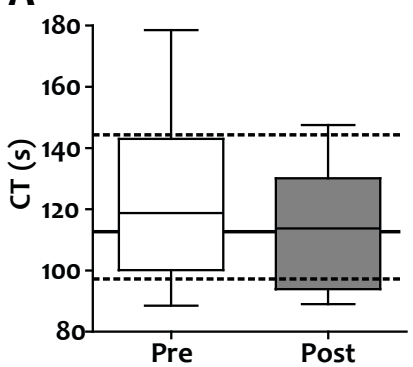

C

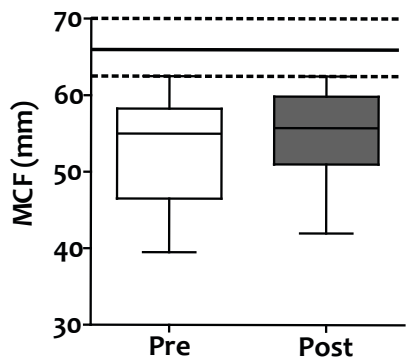

B

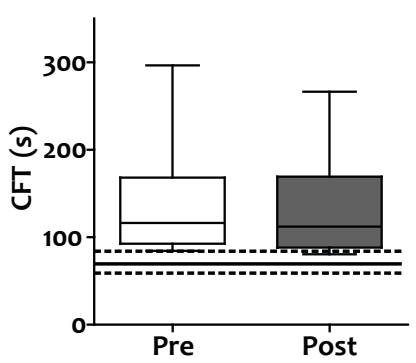

D

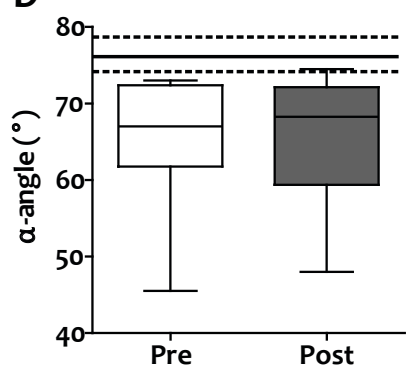

Figure 1. Unchanged fibrin clot elasticity in patient blood after desmopressin treatment. Blood from patients subjected to perioperative dilution and treated with desmopressin was used to measure thromboelasticity after stimulation with tissue factor $/ \mathrm{CaCl}_{2}$. Curve parameters are given of samples taken before and after treatment with desmopressin: clotting time (CT) (A), clot formation time (CFT) (B), maximum clot firmness (MCF) (C), and $\alpha$-angle of rate of clot formation (D). Data are represented as medians \pm interquartile ranges (whiskers show minimum-maximum, $n=12$ ). Lines (dotted) indicate median (interquartile) normal ranges for this patient group before surgery. ${ }^{29}$ 
A
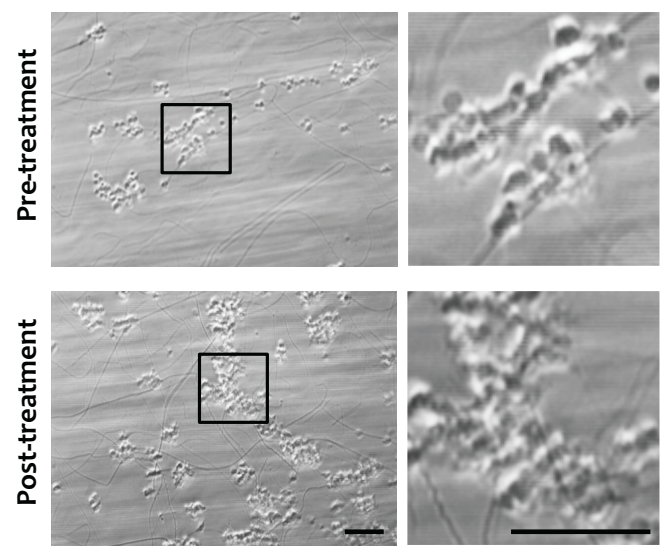

C
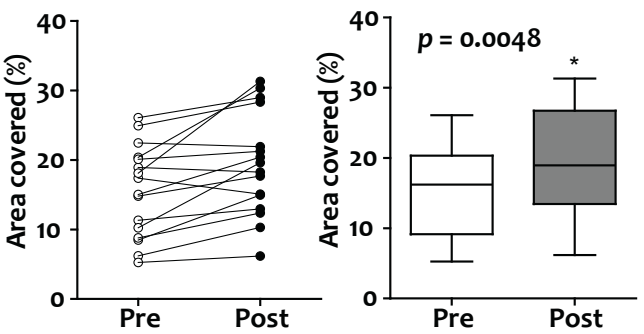

B
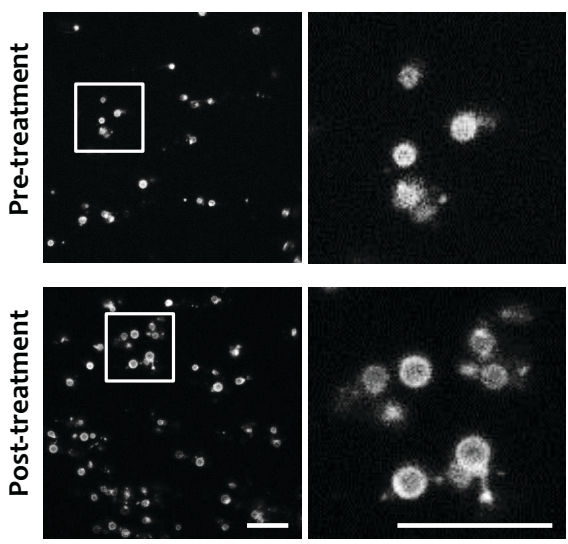

D
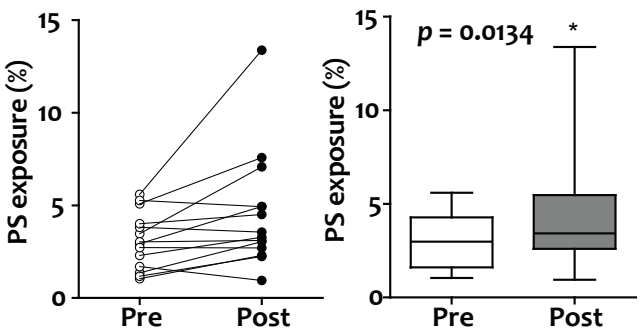

Figure 2. Improved platelet activation and thrombus formation in blood from patients after desmopressin treatment. Whole blood from patients subjected to perioperative dilution, before and after desmopressin treatment, was used for assessment of thrombus formation. Blood samples were perfused for 4 minutes over collagen at a wall shear rate of $1,000 \mathrm{~s}^{-1}$; exposure of phosphatidylserine (PS) on platelets was determined by staining with fluorescein isothiocyanate-annexin A5. (A, B) Representative phase-contrast images (A) and fluorescence images (B) of thrombi formed with pretreatment and posttreatment samples (bars, $20 \mu \mathrm{m}$ ). (C, D) Quantification of surface area coverage of adhered platelets (C) and of PS-positive platelets (D). Data are presented per patient and as medians \pm interquartile ranges (whiskers show minimum-maximum); ${ }^{*} p<0.05(n=14-16)$.

procoagulantphospholipids were present in the assay (Table 2). Interestingly, thrombin peak heights (with phospholipids) of all patient plasmas were below the earlier set threshold of low thrombin generation associated with perioperative bleeding. ${ }^{20}$

Elastic fibrin clot formation in whole blood was determined by thromboelastometry, given the frequent use of this test to assess dilutional coagulopathy. Curve parameters, with the exception of clotting time, of pretreatment samples from all patients were aberrant in comparison with a previous presurgery reference group. ${ }^{29}$ No significant difference was seen when pretreatment and posttreatment samples were compared (Fig. 1). Taken together, these findings indicate that, in these patients with perioperative dilution and bleeding, desmopressin treatment does not result in a coagulation-stimulating effect. 


\section{Effect of desmopressin treatment on thrombus formation on collagen under flow}

As a process depending on the level and functional activity of $\mathrm{VWF},{ }^{41}$ collagendependent thrombus formation under high shear flow conditions was assessed for the whole blood samples taken before treatment and after treatment. Only single platelets and small aggregates adhered to the collagen surface in the case of pretreatment blood samples (Fig. 2A). For essentially all posttreatment samples, platelet adhesion was increased and thrombus formation was enhanced (Fig. 2A, C). The overall median surface area coverage increased from $16.5 \%$ (IQR 9.9-20.8\%) to $19.0 \%$ (IQR $14.5-23.6 \%)(p=0.005)$. Phosphatidylserine exposure on platelets in thrombi was assessed as a measure of glycoprotein VI-induced platelet procoagulant activity (Fig. 2B, D). After desmopressin treatment, this activation marker significantly increased from $3.0 \%$ (IQR $1.8-4.0 \%$ ) to $3.4 \%$ (IQR 2.8-4.9\%) ( $p=0.013$ ).

Correlational analysis suggested that the VWF level was a key determinant of thrombus formation (Fig. 3). For all patient samples together, VWF antigen levels correlated with the surface area coverage $(r=0.624, p<0.001)$ and with phosphatidylserine exposure $(r=0.423, p=0.031)$. As some of the bleeding patients were pretransfused with packed red blood cells, and the haematocrit can influence the process of thrombus formation, ${ }^{28}$ we also compared platelet deposition for patients who did or did not receive red cells. However, no discriminative effect of

A
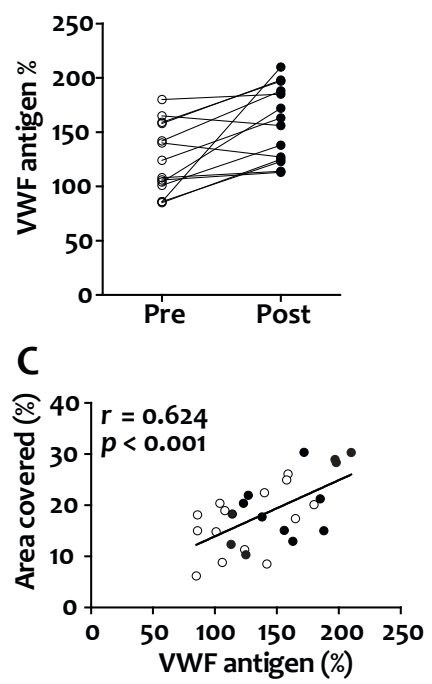

B

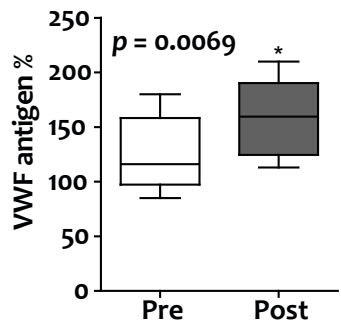

D

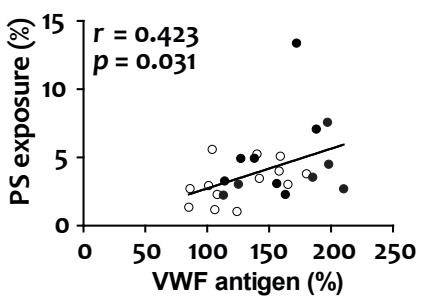

Figure 3. Plasma von Willebrand factor (VWF) concentration as a determinant of thrombus formation and platelet activation. (A, B) Blood samples from patients subjected to perioperative dilution, before (open dots) and after (closed dots) desmopressin treatment, were assessed for VWF antigen level. Data are presented per patient $(\mathbf{A})$ and as medians \pm interquartile ranges ([B]; whiskers show minimummaximum). (C, D) Correlations between VWF antigen levels and parameters of thrombus formation, as in Fig. 2, with calculated Pearson correlation coefficients of VWF level and platelet deposition (C) or phosphatidylserine exposure (D). ${ }^{*} p<0.05(n=14-16)$. 
A

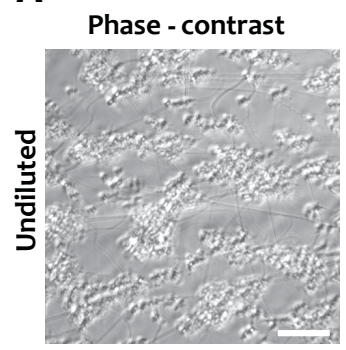

FITC - $\alpha-$ CD62P mAb

AF647 - annexin A5
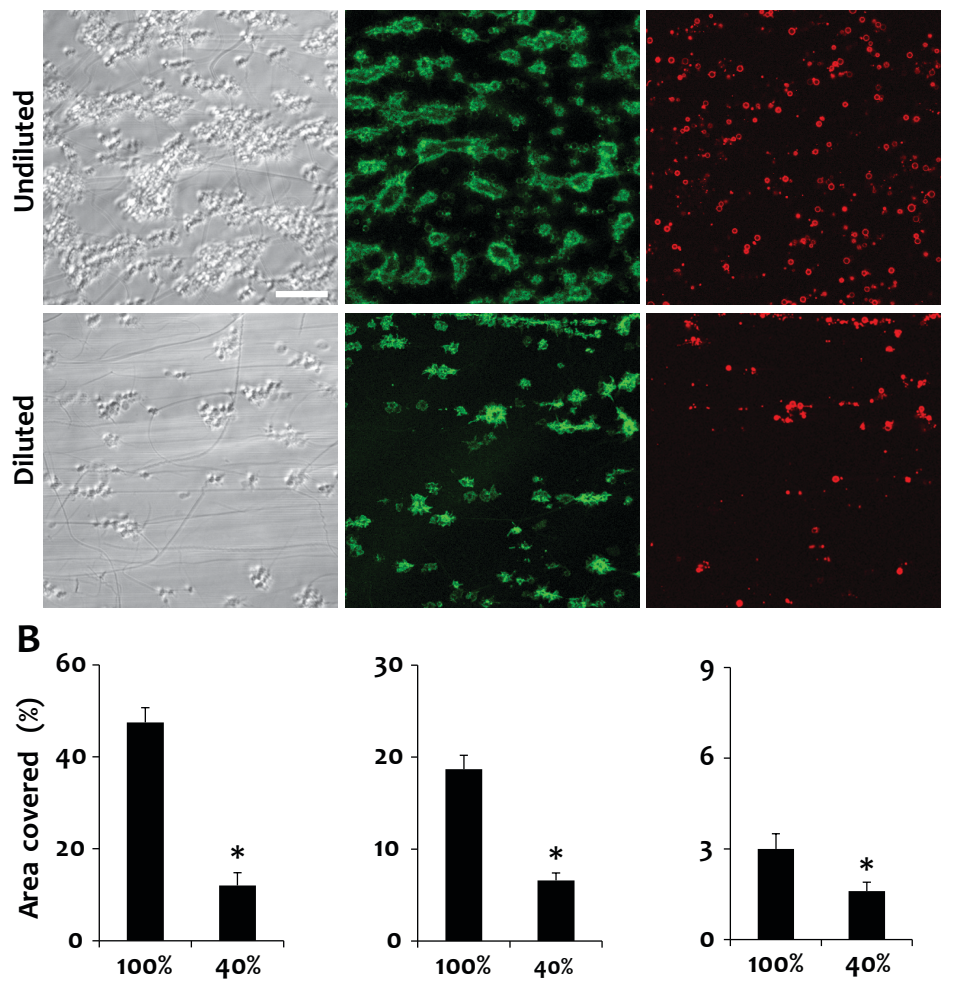

Figure 4. Effect of dilution on thrombus formation and platelet activation under flow. Citrated blood from healthy volunteers was diluted to $40 \%$ with saline medium (see Materials and methods) or remained undiluted, anticoagulated with D-phenylalanyl-L-prolyl-L-arginine chloromethyl ketone/fragmin, and recalcified during perfusion. Flow over collagen took place for 4 minutes at a wall shear rate of 1,000 s'-1, and thrombus formation was assessed as in Figure 2. (A) Representative microscopic images of platelet deposition (phase-contrast), staining for P-selectin (fluorescein isothiocyanate [FITC]-anti-CD62P mAb), and phosphatidylserine exposure (Alexa Fluor [AF]-647-annexin A5; bar, $50 \mu \mathrm{m}$ ). (B) Quantification of images. Means \pm SEM, ${ }^{*} p<0.05(n=3)$.

the red cells was found $(p=0.81)$. This is in line with a (pairwise) statistical analysis demonstrating that haemoglobin levels were not significantly different between pretreatment and posttreatment samples. The change in VWF level after desmopressin treatment was not related to the change in red cell count $(r=0.19, p=$ 0.327 ), indicating that VWF is an independent variable in the effect of desmopressin. Furthermore, partial correlation analysis demonstrated that the VWF level was a significant predictor of of platelet surface coverage that was independent of a change in haematocrit or platelet count $(r=0.605, p<0.001$, and $r=0.613, p<0.001$, after correction for change in haemoglobin level and platelet count, respectively). 
Role of VWF in collagen-dependent thrombus formation under dilution

Subsequent studies with normal blood were carried out to confirm the modulatory effect of changes in VWF level on thrombus formation and platelet activation under conditions of dilution. In vitro dilution of blood, while physiologic divalent cation conditions were maintained, resulted in reduced platelet deposition and small thrombi on collagen, as well as in a reduced number of platelets exposing P-selectin and phosphatidylserine (Fig. 4A, B). To demonstrate the involvement of VWF in this process, diluted blood samples were pretreated with the VWF-neutralising $\mathrm{mAb}$ $82 \mathrm{D} 6 \mathrm{~A} 3$, which binds to the VWF $\mathrm{A} 3$ domain and thereby blocks its interaction with collagen. ${ }^{33,34}$ The $82 \mathrm{D} 6 \mathrm{~A} 3$ antibody resulted in a significant (20\%) decrease in platelet deposition on collagen (Fig. 5A, B). Staining with annexin A5 indicated that the antibody caused a $53 \% \pm 4 \%$ (mean \pm standard error of the mean, $n=3$ ) reduction in phosphatidylserine exposure. On the other hand, addition of VWF concentrate enhanced thrombus formation, an effect that was antagonised by the $82 \mathrm{D} 6 \mathrm{~A} 3$ antibody.

\section{Effect of desmopressin on accumulation of high molecular weight VWF multimers}

Given that the VWF multimer size is an important determinant of its haemostatic potential, ${ }^{13}, 42,43$ we also evaluated the effect of desmopressin treatment on the multimer distribution in the patient plasmas. Horizontal discontinuous agarose gel electrophoresis and western blotting indicated the presence of low, intermediate and

A
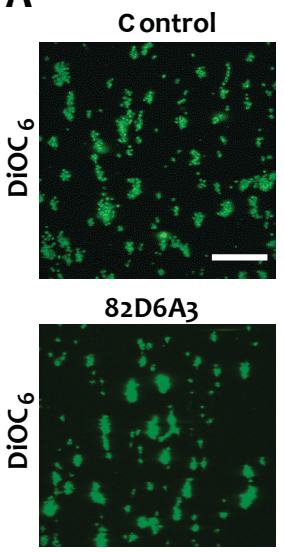

+ VWF

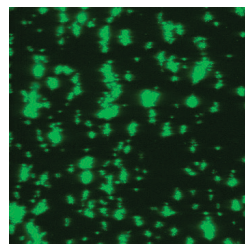

$82 \mathrm{D} 6 \mathrm{~A} 3+\mathrm{VWF}$

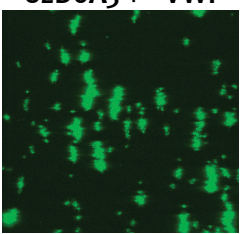

B

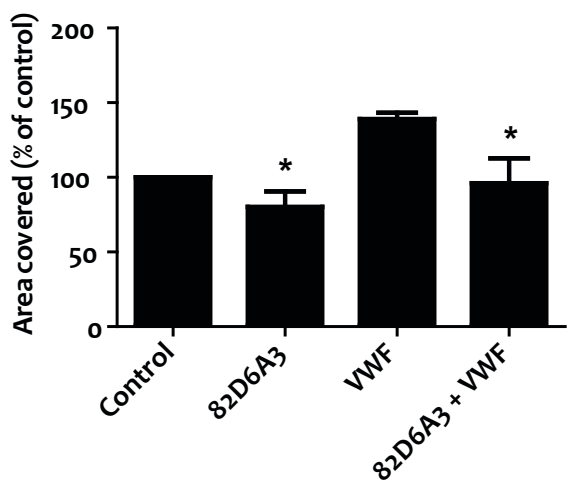

Figure 5. Thrombus formation under conditions of dilution is dependent on von Willebrand factor (VWF) concentration. Blood from healthy volunteers was prelabelled with $\mathrm{DiOC}_{6}$, diluted to $40 \%$ with saline medium, and used for assessment of thrombus formation as in Figure 2. Samples were pretreated with (combinations of) $82 \mathrm{D} 6 \mathrm{~A} 3 \mathrm{mAb}(20 \mu \mathrm{g} / \mathrm{mL})$ and/or VWF concentrate $(150 \mathrm{lU} / \mathrm{mL})$. Perfusion over collagen took place for 4 minutes at a wall shear rate of 1,000 $\mathrm{s}^{-1}$. (A) Representative fluorescence images of DiOC $_{6}$-stained thrombi after flow (bar, $50 \mu \mathrm{m}$ ). (B) Quantification of surface area coverage of adhered platelets. Means \pm SEM, ${ }^{*} p<0.05(n=3)$. 
A

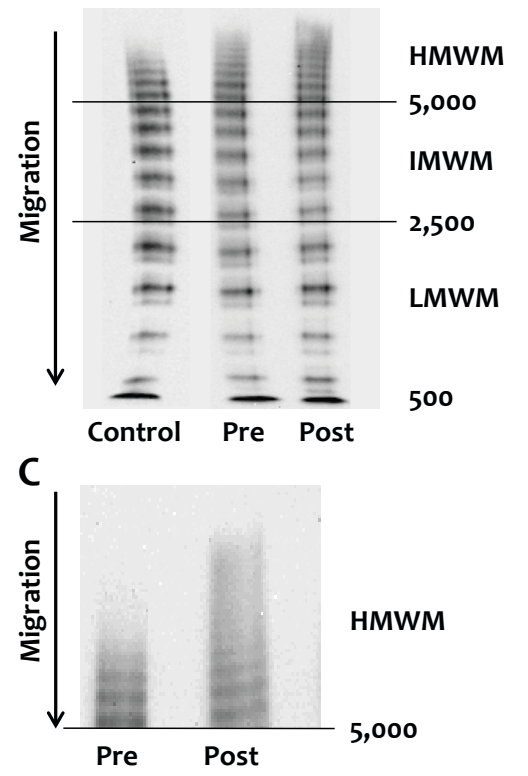

B
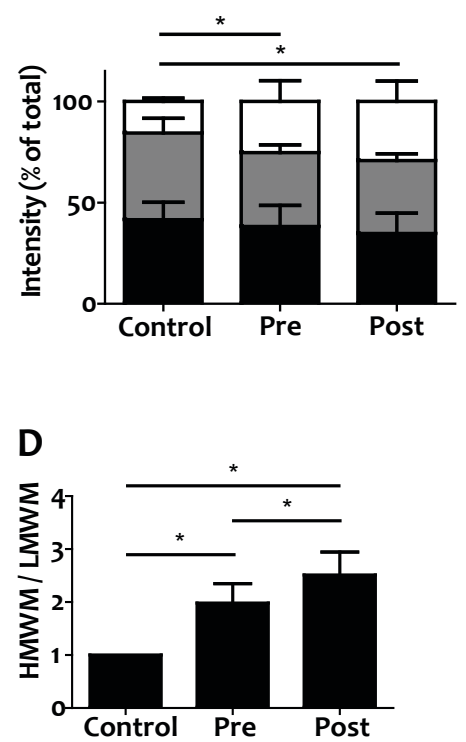

Figure 6. Increase in the level of high molecular weight von Willebrand factor (VWF) multimers (HMWMs) in patient blood after desmopressin treatment. The composition of VWF multimers was assessed in plasmas from patients before and after desmopressin treatment. All samples were analysed after dilution to $0.05 \mathrm{IU} / \mathrm{mL}$ VWF antigen. Standard human plasma, diluted to $0.05 \mathrm{IU} / \mathrm{mL} \mathrm{VWF}$ antigen, served as a control. (A) Western blot of control plasma and representative patient plasmas before and after treatment. Positions on blots indicate HMWMs, intermediate molecular weight multimers (IMWMs), and low molecular weight multimers (LMWMs) (kDa). (B) Quantification of relative distributions of VWF multimer classes in control and patient plasmas (black, LMWM; grey, IMWM; white, HMWM). (C, D) Western blot of the HMWM part of a representative patient plasma before and after treatment (C), and quantification of the HMWM/LMWM ratio (D). Means \pm SD, ${ }^{*} p<0.05(n=13)$.

high molecular weight multimers in both standard control and patient plasma samples (Fig. 6A). The proportion of high molecular weight multimers (i.e. > 5,000 kDa) was substantially higher in pretreatment (26.4\%; IQR 15.9-33.4\%) and posttreatment (30.3 \%; IQR 25.6-35.4\%) samples than in standard control plasma (15.0\%; IQR 14.6-15.5\%; $p<$ $0.001)$ Fig. 6B). Chi-square analysis of the multimer distribution pattern demonstrated a significant difference between control samples on the one hand, and pretreatment $\left(\chi^{2} 8.34 ; p=0.02\right)$ and posttreatment $\left(\chi^{2} 13.64 ; p=0.001\right)$ samples on the other hand.

Higher-resolution blots were run to further separate the high molecular weight multimers. These indicated prominent accumulation in the largest bands after desmopressin treatment (Fig. 6C). Calculation of the ratio of high to low molecular weight multimers showed a significant rise in the pretreatment samples in comparison with standard plasma, an effect that was further increased in the posttreatment samples (Fig. 6D). 


\section{Discussion}

In the present study, we provide the first evidence that desmopressin treatment of patients with dilution and postsurgical bleeding induces a substantial increase in the level of high molecular weight multimers of VWF above an already elevated VWF antigen level as compared with other coagulation factors. This increase in the level of high molecular weight VWF multimers results in improved platelet deposition and thrombus formation on collagen under flow, as well as in a net increase in platelet procoagulant activity on collagen. It is reasonable to assume that the increased VWF-dependent platelet activity contributes to the improved haemostasis in these patients. Indeed, VWF and, in particular, the high molecular weight multimers have an accepted role in haemostasis. ${ }^{10}$ In addition, it is accepted that collagen-dependent thrombus formation can reflect haemostatic activity. ${ }^{44}$

Previous reports, restricted to desmopressin infusion in healthy individuals, have pointed to an accumulation of larger VWF multimers after 0.5-24 hours of treatment. ${ }^{45,46}$ The present results are remarkable in this light, as they point to an extra functional effect of desmopressin on top of the already elevated VWF levels after surgery in these patients ( $120 \%$ of normal), most likely because of procedurerelated endothelial activation. It is of note that further analysis of the data revealed a trend for correlation of pretreatment VWF antigen levels and the difference in VWF levels before and after treatment $(r=0.461, p=0.073)$, suggesting that the elevation in VWF level after treatment is less pronounced in patients with the highest VWF levels before treatment. In comparison, prothrombin levels in these patients are $55 \%$ of normal, which is indicative of massive dilution with crystalloids and colloids during surgery. ${ }^{28,29}$ These findings are compatible with the evidence that surgery in combination with connection to extracorporeal circulation leads to activation of endothelial cells and platelets, and the release of VWF into the circulation..$^{38}$

A typical finding was that desmopressin treatment led to a greater increase in FVIII (median from $93 \%$ to $177 \%$ ) than of VWF (from $116 \%$ to $160 \%$ ). In addition, the levels of both factors were already higher at baseline than that of prothrombin (median $53 \%$ ), i.e. $93 \%$ (FVIII) and $116 \%$ (VWF). Hence, it appears that the FVIII level, in part, follows the VWF level, which is in agreement with the evidence that FVIII is an acute-phase protein, being released from liver and endothelial cells. ${ }^{47}$ Probably, the posttreatment change in FVIII is attributable to de novo release. However, our data do not show whether the new release is triggered by: (i) elevation of VWF by itself, protecting FVIII from proteolytic degradation; (ii) a stimulating effect of desmopressin on the FVIII-releasing cells; or (iii) the poor haemostatic state of the patients.

Western blot analysis showed that, in comparison with standard control plasma, the contribution of high molecular weight multimers of VWF is elevated in patients prior to treatment, and further increased after desmopressin treatment. It has been proposed that, in patients with severe aortic stenosis, levels of high molecular weight VWF multimers are reduced prior to surgery, without an effect on postoperative 
blood loss. ${ }^{48}$ However, in our study, with only a few stenotic patients, we could not detect specific changes in multimer levels. Earlier work showed that the larger triplet bands of VWF are more active in recruiting platelets to collagen-bound VWF under flow conditions. ${ }^{41}$ In the present patient samples, no obvious change occurred in the VWF triplet distribution.

The enhanced thrombus formation after desmopressin treatment was accompanied by an increased number of procoagulant platelets on the collagen surface. This result is in line with earlier indications that desmopressin treatment: (i) leads to an increase in the number of procoagulant collagen-activated and thrombinactivated (COAT) platelets, as assessed by flow cytometry; ${ }^{49}$ and (ii) can restore the effect of patient treatment with Aspirin or integrin $\alpha_{11 b} \beta_{3}$ blockers. ${ }^{50,51}$ Previous results have indicated that platelet aggregation and thrombus formation under flow depend on the presence and activity of VWF at poststenotic sites, owing to fluid mechanical conditions. ${ }^{32,52}$ One study did not find increased thrombus formation with blood from patients who received desmopressin..$^{53}$ This discrepancy is most likely attributable to the low shear rate $\left(310 \mathrm{~s}^{-1}\right)$ applied in that study, thus diminishing the dependency of thrombus growth on VWF.

Earlier, it was suggested that, in patients with dilutional coagulopathy, the combined measurement of thrombin generation and fibrin clot formation provides a better prediction of bleeding than either test alone. ${ }^{20}$ Interestingly, for the patients in our study, almost all parameters of thrombin generation and thromboelastometry were in the ranges that were previously associated with bleeding. No effect of desmopressin treatment was found with either assay, indicating that the coagulation state was unaltered. However, the data suggest that VWF levels, although not below $100 \%$ before treatment, can also be considered as a limiting factor in the haemostatic process, as the desmopressin-induced elevation in (high molecular weight) VWF promotes platelet functions in thrombus formation and procoagulant activity, and postoperative bleeding stopped in 14 of 16 patients. It is worth noting that disadvantageous effects of desmopressin have been reported, albeit mostly transient and mild, such as mild tachycardia, headache, and facial flushing. ${ }^{54} \mathrm{~A}$ rare, serious side effect in patients with cardiovascular disease is a moderately increased risk of arterial thrombotic effects, i.e. stroke or myocardial infarction, as reviewed by Levi et al..$^{55}$

Some caution is needed here, as the group of patients receiving desmopressin because of postoperative bleeding is, by nature, a heterogeneous population, with many of the patients requiring blood component transfusion prior to desmopressin treatment. Importantly, in the present data, the correlation between VWF antigen levels and platelet surface coverage was not influenced by changes in platelet or haemoglobin levels. This strongly suggests that the improved thrombus formation after treatment is attributable to the consistent change in VWF rather than to small changes in platelet or erythrocyte numbers. For the patient group as a whole, parameters of thrombin generation and thromboelastometry remained unchanged after desmopressin treatment. On the other hand, in some of the patients with 
cessation of bleeding, levels of VWF and coagulation parameters did increase. Hence, improvement of VWF-dependent platelet function in combination with a normalised coagulant potential might be a preferred way to repair haemostatic insufficiency in these patients. A systematic overview on the use of desmopressin in open heart surgery concluded that desmopressin reduces postoperative bleeding particularly in patients with large blood losses, but also that this effect is limited. ${ }^{23}$ The present results suggest that the limitation could result from the additional need to restore thrombin and fibrin generation.

Taken together, these data show, for the first time, that desmopressin treatment after cardiothoracic surgery increases the levels of high molecular weight VWF multimers, leading to enhancement of thrombus formation and platelet activation under flow conditions. This can help to restore haemostasis in these patients.

\section{Authorship contribution}

P. E. J. van der Meijden and J. W. M. Heemskerk are the principal investigators and take primary responsibility for the paper. M. D. Lancé and L. P. J. Verheijen treated and recruited the patients. F. Swieringa, B. Fuchs, M. A. H. Feijge, L. P. J. Verheijen, K. R. Hughes, R. van Oerle, and B. A. Solecka performed analytic work. F. Swieringa, M. A. H. Feijge, and P. E. J. van der Meijden performed the statistical analysis. H. Deckmyn provided essential materials. F. Swieringa, C. Kannicht, J. W. M. Heemskerk, and P. E. J. van der Meijden coordinated the research. F. Swieringa, J. W. M. Heemskerk, and P. E. J. van der Meijden wrote the paper.

\section{Acknowledgements}

This research was supported by the Dutch Landsteiner Foundation for Blood Transfusion Research (1006) and the Cardiovascular Center Maastricht. We thank G. J. Kuiper and P. Verhezen for experimental and analytical support.

\section{Disclosure of conflict of interests}

B. Fuchs, B. A. Solecka and C. Kannicht are employees of Octapharma R\&D (Department of Molecular Biochemistry), Berlin, Germany. M. D. Lancé reports receiving grants from the Dutch Landsteiner Foundation for Blood Transfusion Research and CSL Behring, during the conduct of the study. J. W. M. Heemskerk reports receiving grants from the Dutch Landsteiner Foundation for Blood Transfusion Research, CSL Behring, and the Center for Translational Molecular Medicine, and personal fees from Cardiovascular Center Maastricht, during the conduct of the study. F . Swieringa reports receiving grants from the Dutch Landsteiner Foundation for Blood Transfusion Research, during the conduct of the study. P. E. J. van der Meijden reports receiving grants from the Dutch Landsteiner Foundation for Blood Transfusion Research and personal fees 
from Cardiovascular Center Maastricht, during the conduct of the study. The other authors state that they have no conflict of interest.

\section{References}

1. Rugeri L, Béguin S, Hemker C, Bordet JC, Fleury R, Chatard B, Negrier C, Dargaud Y. Thrombingenerating capacity in patients with von Willebrand's disease. Haematologica. 2007;92:1639-1646.

2. Versteeg HH, Heemskerk JW, Levi M, Reitsma PH. New fundamentals in hemostasis. Physiol Rev. 2013;93:327-358.

3. Weiss HJ, Sussman, II, Hoyer LW. Stabilization of factor VIII in plasma by the von Willebrand factor. Studies on posttransfusion and dissociated factor VIII and in patients with von Willebrand's disease. J Clin Invest. 1977;60:390-404.

4. Keularts IM, Hamulyák K, Hemker HC, Béguin S. The effect of DDAVP infusion on thrombin generation in platelet-rich plasma of von Willebrand type 1 and in mild haemophilia $A$ patients. Thromb Haemost. 2000;84:638-642.

5. Keuren JF, Ulrichts H, Feijge MA, Hamulyák K, Deckmyn H, Lindhout T, Heemskerk JW. Integrin $a_{11 b} \beta_{3}$ and shear-dependent action of glycoprotein lba stimulate platelet-dependent thrombin formation in stirred plasma. J Lab Clin Med. 2003;141:350-358.

6. Vanschoonbeek K, Feijge MA, Van Kampen RJ, Kenis H, Hemker HC, Giesen PL, Heemskerk JW. Initiating and potentiating role of platelets in tissue factor-induced thrombin generation in the presence of plasma: subject-dependent variation in thrombogram characteristics. J Thromb Haemost. 2004;2:476-484.

7. Valentijn KM, Sadler JE, Valentijn JA, Voorberg J, Eikenboom J. Functional architecture of WeibelPalade bodies. Blood. 2011;117:5033-5043.

8. Rondaij MG, Bierings R, Kragt A, Van Mourik JA, Voorberg J. Dynamics and plasticity of WeibelPalade bodies in endothelial cells. Arterioscler Thromb Vasc Biol. 2006;26:1002-1007.

9. Wagner DD, Fay PJ, Sporn LA, Sinha S, Lawrence SO, Marder VJ. Divergent fates of von Willebrand factor and its propolypeptide (von Willebrand antigen II) after secretion from endothelial cells. Proc Natl Acad Sci U S A. 1987;84:1955-1959.

10. Stockschlaeder M, Schneppenheim R, Budde U. Update on von Willebrand factor multimers: focus on high-molecular-weight multimers and their role in hemostasis. Blood Coagul Fibrinolysis. 2014;25:206-216.

11. De Ceunynck K, De Meyer SF, Vanhoorelbeke K. Unwinding the von Willebrand factor strings puzzle. Blood. 2013;121:270-277.

12. Rybaltowski M, Suzuki Y, Mogami H, Chlebinska I, Brzoska T, Tanaka A, Banno F, Miyata T, Urano T. In vivo imaging analysis of the interaction between unusually large von Willebrand factor multimers and platelets on the surface of vascular wall. Pflügers Arch. 2011;461:623-633.

13. Schneider SW, Nuschele S, Wixforth A, Gorzelanny C, Alexander-Katz A, Netz RR, Schneider MF. Shear-induced unfolding triggers adhesion of von Willebrand factor fibers. Proc Natl Acad Sci U S A. 2007;104:7899-7903.

14. Budde U, Pieconka A, Will K, Schneppenheim R. Laboratory testing for von Willebrand disease: contribution of multimer analysis to diagnosis and classification. Semin Thromb Hemost. 2006;32:514-521.

15. Reininger AJ. Function of von Willebrand factor in haemostasis and thrombosis. Haemophilia. 2008;14 Suppl 5:11-26.

16. Svensson PJ, Bergqvist PB, Juul KV, Berntorp E. Desmopressin in treatment of haematological disorders and in prevention of surgical bleeding. Blood Rev. 2014;28:95-102.

17. Wang JW, Bouwens EA, Pintao MC, Voorberg J, Safdar H, Valentijn KM, De Boer HC, Mertens $\mathrm{K}$, Reitsma PH, Eikenboom J. Analysis of the storage and secretion of von Willebrand factor in 
blood outgrowth endothelial cells derived from patients with von Willebrand disease. Blood. 2013;121:2762-2772.

18. Davidson S. State of the art - how I manage coagulopathy in cardiac surgery patients. Br J Haematol. 2014;164:779-789.

19. Paparella D, Brister SJ, Buchanan MR. Coagulation disorders of cardiopulmonary bypass: a review. Intensive Care Med. 2004;30:1873-1881.

20. Schols SE, Lancé MD, Feijge MA, Damoiseaux J, Marcus MA, Hamulyák K, Ten Cate H, Heemskerk JW, Van Pampus EC. Impaired thrombin generation and fibrin clot formation in patients with dilutional coagulopathy during major surgery. Thromb Haemost. 2010;103:318-328.

21. Despotis GJ, Levine V, Saleem R, Spitznagel E, Joist JH. Use of point-of-care test in identification of patients who can benefit from desmopressin during cardiac surgery: a randomised controlled trial. Lancet. 1999;354:106-110.

22. Weber CF, Dietrich W, Spannagl M, Hofstetter C, Jambor C. A point-of-care assessment of the effects of desmopressin on impaired platelet function using multiple electrode whole-blood aggregometry in patients after cardiac surgery. Anesth Analg. 2010;110:702-707.

23. Cattaneo M. The use of desmopressin in open-heart surgery. Haemophilia. 2008;14 Suppl 1:40-47.

24. Pleym H, Stenseth R, Wahba A, Bjella L, Tromsdal A, Karevold A, Dale O. Prophylactic treatment with desmopressin does not reduce postoperative bleeding after coronary surgery in patients treated with aspirin before surgery. Anesth Analg. 2004;98:578-584.

25. Van der Meijden PE, Feijge MA, Giesen PL, Huijberts M, Van Raak LP, Heemskerk JW. Platelet P2 $Y_{12}$ receptors enhance signalling towards procoagulant activity and thrombin generation. A study with healthy subjects and patients at thrombotic risk. Thromb Haemost. 2005;93:1128-1136.

26. Chen D, Tange JI, Meyers BJ, Pruthi RK, Nichols WL, Heit JA. Validation of an automated latex particle-enhanced immunoturbidimetric von Willebrand factor activity assay. J Thromb Haemost. 2011;9:1993-2002.

27. Hemker HC, Giesen P, Al Dieri R, Regnault V, De Smedt E, Wagenvoord R, Lecompte T, Béguin S. Calibrated automated thrombin generation measurement in clotting plasma. Pathophysiol Haemost Thromb. 2003;33:4-15.

28. Schols SE, Feijge MA, Lancé MD, Hamulyák K, Ten Cate H, Heemskerk JW, Van Pampus EC. Effects of plasma dilution on tissue-factor-induced thrombin generation and thromboelastography: partly compensating role of platelets. Transfusion. 2008;48:2384-2394.

29. Ninivaggi M, Feijge MA, Baaten CC, Kuiper GJ, Marcus MA, Ten Cate H, Lancé MD, Heemskerk JW, Van der Meijden PE. Additive roles of platelets and fibrinogen in whole-blood fibrin clot formation upon dilution as assessed by thromboelastometry. Thromb Haemost. 2014;111:447-457.

30. Van Kruchten R, Cosemans JM, Heemskerk JW. Measurement of whole blood thrombus formation using parallel-plate flow chambers - a practical guide. Platelets. 2012;23:229-242.

31. Gilio K, Van Kruchten R, Braun A, Berna-Erro A, Feijge MA, Stegner D, Van der Meijden PE, Kuijpers MJ, Varga-Szabo D, Heemskerk JW, Nieswandt B. Roles of platelet STIM1 and Orai1 in glycoprotein VI- and thrombin-dependent procoagulant activity and thrombus formation. $J$ Biol Chem. 2010;285:23629-23638.

32. Westein E, Van der Meer AD, Kuijpers MJ, Frimat JP, Van den Berg A, Heemskerk JW. Atherosclerotic geometries exacerbate pathological thrombus formation poststenosis in a von Willebrand factordependent manner. Proc Natl Acad Sci U S A. 2013;110:1357-1362.

33. Bonnefoy A, Romijn RA, Vandervoort PA, Van Rompaey I, Vermylen J, Hoylaerts MF. von Willebrand factor $A 1$ domain can adequately substitute for $A 3$ domain in recruitment of flowing platelets to collagen. J Thromb Haemost. 2006;4:2151-2161.

34. Vanhoorelbeke K, Depraetere H, Romijn RA, Huizinga EG, De Maeyer M, Deckmyn H. A consensus tetrapeptide selected by phage display adopts the conformation of a dominant discontinuous epitope of a monoclonal anti-VWF antibody that inhibits the von Willebrand factor-collagen 
interaction. J Biol Chem. 2003;278:37815-37821.

35. Munnix IC, Gilio K, Siljander PR, Raynal N, Feijge MA, Hackeng TM, Deckmyn H, Smethurst PA, Farndale RW, Heemskerk JW. Collagen-mimetic peptides mediate flow-dependent thrombus formation by high- or low-affinity binding of integrin $\alpha_{2} \beta_{1}$ and glycoprotein VI. J Thromb Haemost. 2008;6:2132-2142.

36. De Witt SM, Swieringa F, Cavill R, Lamers MM, Van Kruchten R, Mastenbroek T, Baaten C, Coort S, Pugh N, Schulz A, Scharrer I, Jurk K, Zieger B, Clemetson KJ, Farndale RW, Heemskerk JW, Cosemans JM. Identification of platelet function defects by multi-parameter assessment of thrombus formation. Nat Commun. 2014;5:4257.

37. Refaai MA, Van Cott EM, Lukoszyk M, Hughes J, Eby CS. Loss of factor VIII and von Willebrand factor activities during cold storage of whole blood is reversed by rewarming. Lab Hematol. 2006;12:99-102.

38. Panagiotopoulos I, Palatianos G, Michalopoulos A, Chatzigeorgiou A, Prapas S, Kamper EF. Alterations in biomarkers of endothelial function following on-pump coronary artery revascularization. J Clin Lab Anal. 2010;24:389-398.

39. Béguin S, Keularts I, Al Dieri R, Bellucci S, Caen J, Hemker HC. Fibrin polymerization is crucial for thrombin generation in platelet-rich plasma in a VWF-GPIb-dependent process, defective in Bernard-Soulier syndrome. J Thromb Haemost. 2004;2:170-176.

40. Schols SE, Van der Meijden PE, Van Oerle R, Curvers J, Heemskerk JW, Van Pampus EC. Increased thrombin generation and fibrinogen level after therapeutic plasma transfusion: relation to bleeding. Thromb Haemost. 2008;99:64-70.

41. Fuchs B, De Witt S, Solecka BA, Kroning M, Obser T, Cosemans JM, Schneppenheim R, Heemskerk JW, Kannicht C. Distinct role of von Willebrand factor triplet bands in glycoprotein Ib-dependent platelet adhesion and thrombus formation under flow. Semin Thromb Hemost. 2013;39:306-314.

42. Federici AB, Bader R, Pagani S, Colibretti ML, De Marco L, Mannucci PM. Binding of von Willebrand factor to glycoproteins Ib and IIb/IIla complex: affinity is related to multimeric size. $\mathrm{Br} J$ Haematol. 1989;73:93-99.

43. Fischer BE, Kramer G, Mitterer A, Grillberger L, Reiter M, Mundt W, Dorner F, Eibl J. Effect of multimerization of human and recombinant von Willebrand factor on platelet aggregation, binding to collagen and binding of coagulation factor VIII. Thromb Res. 1996;84:55-66.

44. Morowski M, Vögtle T, Kraft P, Kleinschnitz C, Stoll G, Nieswandt B. Only severe thrombocytopenia results in bleeding and defective thrombus formation in mice. Blood. 2013;121:4938-4947.

45. Batlle J, Lopez-Fernandez MF, Lopez-Borrasca A, Lopez-Berges C, Dent JA, Berkowitz SD, Ruggeri ZM, Zimmerman TS. Proteolytic degradation of von Willebrand factor after DDAVP administration in normal individuals. Blood. 1987;70:173-176.

46. Ruggeri ZM, Mannucci PM, Lombardi R, Federici AB, Zimmerman TS. Multimeric composition of factor VIII/von Willebrand factor following administration of DDAVP: implications for pathophysiology and therapy of von Willebrand's disease subtypes. Blood. 1982;59:1272-1278.

47. Kaufmann JE, Vischer UM. Cellular mechanisms of the hemostatic effects of desmopressin (DDAVP). J Thromb Haemost. 2003;1:682-689.

48. Bolliger D, Dell-Kuster S, Seeberger MD, Tanaka KA, Gregor M, Zenklusen U, Tsakiris DA, Filipovic M. Impact of loss of high-molecular-weight von Willebrand factor multimers on blood loss after aortic valve replacement. Br J Anaesth. 2012;108:754-762.

49. Colucci G, Stutz M, Rochat S, Conte T, Pavicic M, Reusser M, Giabbani E, Huynh A, Thurlemann C, Keller P, Alberio L. The effect of desmopressin on platelet function: a selective enhancement of procoagulant COAT platelets in patients with primary platelet function defects. Blood. 2014;123:1905-1916.

50. Ranucci M, Nano G, Pazzaglia A, Bianchi P, Casana R, Tealdi DG. Platelet mapping and desmopressin reversal of platelet inhibition during emergency carotid endarterectomy. J Cardiothorac Vasc 
Anesth. 2007;21:851-854.

51. Reiter RA, Mayr F, Blazicek H, Galehr E, Jilma-Stohlawetz P, Domanovits H, Jilma B. Desmopressin antagonizes the in vitro platelet dysfunction induced by GPIIb/IIla inhibitors and aspirin. Blood. 2003;102:4594-4599.

52. Ogawa S, Ohnishi T, Hosokawa K, Szlam F, Chen EP, Tanaka KA. Haemodilution-induced changes in coagulation and effects of haemostatic components under flow conditions. $\mathrm{Br} J$ Anaesth. 2013;111:1013-1023.

53. Calmer S, Ferkau A, Larmann J, Johanning K, Czaja E, Hagl C, Echtermeyer F, Goudeva L, Heuft HG, Theilmeier G. Desmopressin (DDAVP) improves recruitment of activated platelets to collagen but simultaneously increases platelet endothelial interactions in vitro. Platelets. 2014;25:8-15.

54. Ozgonenel B, Rajpurkar M, Lusher JM. How do you treat bleeding disorders with desmopressin? Postgrad Med J. 2007;83:159-163.

55. Levi M, Cromheecke ME, De Jonge E, Prins MH, De Mol BJ, Briët E, Büller HR. Pharmacological strategies to decrease excessive blood loss in cardiac surgery: a meta-analysis of clinically relevant endpoints. Lancet. 1999;354:1940-1947. 



\section{CHAPTER 5}

Key role of integrin $\alpha_{11 b} \beta_{3}$ signalling to Syk kinase in tissue factor-induced thrombin generation

Van der Meijden PE, Feijge MA*, Swieringa F*, Gilio K, Nergiz-Unal R, Hamulyák K, Heemskerk JW (* equal contribution)

Cell Mol Life Sci. 2012;69:3481-3492

Reprinted with permission 


\begin{abstract}
The fibrin(ogen) receptor, integrin $\alpha_{11 \mathrm{~b}} \beta_{3}$, has a well-established role in platelet spreading, aggregation and clot retraction. How $\alpha_{11 b} \beta_{3}$ contributes to plateletdependent coagulation is less well resolved. Here, we demonstrate that the potent suppressing effect of clinically used $\alpha_{11 b} \beta_{3}$ blockers on tissue factor-induced thrombin generation is linked to diminished platelet $\mathrm{Ca}^{2+}$ responses and phosphatidylserine (PS) exposure. The same blockers suppress these responses in platelets stimulated with collagen and thrombin receptor agonists, whereas added fibrinogen potentiates these responses. In platelets spreading on fibrinogen, outside-in $\alpha_{11 b} \beta_{3}$ signalling similarly enhances thrombin-induced $\mathrm{Ca}^{2+}$ rises and PS exposure. These responses are reduced in $\alpha_{11 b} \beta_{3}$-deficient platelets from patients with Glanzmann's thrombasthenia. Furthermore, the contribution of $\alpha_{11 b} \beta_{3}$ to tissue factor-induced platelet $\mathrm{Ca}^{2+}$ rises, PS exposure and thrombin generation in plasma are fully dependent on Syk kinase activity. Tyrosine phosphorylation analysis confirms a key role of Syk activation, which is largely but not exclusively dependent on $\alpha_{111} \beta_{3}$ activation. It is concluded that the majority of tissue factor-induced procoagulant activity of platelets relies on Syk activation and ensuing $\mathrm{Ca}^{2+}$ signal generation, and furthermore that a considerable part of Syk activation relies on $\alpha_{\| 1 b} \beta_{3}$ signalling. These results hence point to a novel role of Syk in integrin-dependent thrombin generation.
\end{abstract}

\title{
Introduction
}

Integrin $\alpha_{11 b} \beta_{3}$ (glycoprotein $\mathrm{Ilb} / \mathrm{llla}$ ) is among the most abundantly expressed glycoproteins at the platelet surface, which strongly regulates the adhesion and aggregation of platelets. Platelet agonists induce a conformational change in the extracellular part of $\alpha_{11 b} \beta_{3}$ via so-called inside-out signalling events, accumulating in Rap1b activation and talin-1 complex formation, which increases the adhesiveness of the integrin. ${ }^{1-3}$ Conversely, binding of $\alpha_{11 b} \beta_{3}$ to its ligands, such as fibrinogen, fibrin and von Willebrand factor, stimulates outside-in signal transduction by a pathway involving activation of Src and Syk protein tyrosine kinases..$^{4-6}$ Platelet responses known to rely on $\alpha_{11 \mathrm{~b}} \beta_{3}$ outside-in signalling include the shedding of microparticles, the formation of lamellipods of fibrinogen-adhered platelets, and the contraction of platelets within a fibrin clot. ${ }^{7-9}$

It has been known for a long time that platelets stimulated with strong agonists promote the processes of thrombin generation and blood coagulation. ${ }^{10,11}$ This procoagulant function is triggered by agonist combinations like collagen/thrombin, which evoke prolonged and high rises in cytosolic $\left[\mathrm{Ca}^{2+}\right]$, and also by stimulating platelets in plasma with tissue factor. ${ }^{12,13}$ The procoagulant activity is caused by exposure of the negatively charged phosphatidylserine (PS), at the membrane surface via a transmembrane protein encoded by TMEM16F, ${ }^{14}$ which promotes the local assembly of vitamin K-dependent coagulation factors and hence generation of 
thrombin. ${ }^{15}$ Early findings have pointed to a significant role of $\alpha_{11 \mathrm{~b}} \beta_{3}$ in the development of platelet procoagulant activity, in that integrin antagonists were found to suppress tissue factor-induced thrombin generation. 13,16,17 Yet, the mechanism by which $\alpha_{11 b} \beta_{3}$ blockage interferes with platelet-dependent coagulation has not been resolved. Suggestions are that the integrin is (i) involved in the formation of procoagulant microparticles, ${ }^{17-19}$ (ii) directly binds prothrombin, ${ }^{20}$ or (iii) provides binding sites for factor Va and other coagulation factors. ${ }^{21,22}$ Studies so far are complicated by data that show that distinct $\alpha_{11 b} \beta_{3}$ antagonists may differ in their effects on platelet activation and procoagulant activity. ${ }^{19,23}$ Another complicating factor is that part of the role of $\alpha_{11 b} \beta_{3}$ may be secondary to that of autocrine ADP, which enhances PS exposure via $\mathrm{P}_{2} \mathrm{Y}_{12}$ receptor stimulation. ${ }^{24-26}$ In this paper, we hypothesise that $\alpha_{11 b} \beta_{3}$ interaction with its principal ligand, fibrinogen, enhances platelet-dependent thrombin generation via an outside-in signalling mechanism. We demonstrate that signalling via Syk kinase is responsible for the majority of tissue factor-induced thrombin generation of platelets in plasma by stimulating PS exposure.

\section{Materials and methods}

\section{Materials}

Human a-thrombin was obtained from Enzyme Research Laboratories, recombinant human tissue factor came from Dade Behring, abciximab (reopro) from Centocor; tirofiban (aggrastat) from Merck Sharp \& Dohme, and eptifibatide (integrilin) from GlaxoSmithKline. Dimethyl BAPTA (DMBAPTA), Fura-2 and Fura-Red acetoxymethyl esters were from Molecular Probes, while Syk inhibitor II and IV were from Merck Biosciences. Apyrase, bovine serum albumin (BSA), human and bovine fibrinogen (fraction 1, type III), and non-radioactive protein tyrosine kinase assay kit were all from Sigma. Cangrelor (AR-C69931MX) was kindly provided by The Medicine Company. Ancrod came from NIBSC; fluorescein isothiocyanate (FITC)-labelled annexin A5 from PharmaTarget, and FITC-labelled monoclonal antibody (mAb) against platelet-bound human fibrinogen from WAK Chemie Medical. Rabbit anti-phospho-Syk (Tyr ${ }^{525 / 526}$ ) $\mathrm{mAb}$ and HRP-linked anti-rabbit lgG were from Cell Signaling Technology, mouse antiSyk mAb was from Santa Cruz Biotechnology, rabbit anti-a-tubulin Ab from Abcam, and HRP-linked anti-mouse IgG from GE Healthcare. Pefabloc (Gly-Pro-Arg-Pro-amide, GPRP) was obtained from Kordia Life Sciences. Microbeads coated with human anti-CD31 mAb and MS columns were a kind gift from Miltenyi Biotec. Procoagulant phospholipid vesicles (PS:phosphatidyl choline:phosphatidyl ethanolamine 1:3:1; mol/ mol) were prepared as described. ${ }^{27}$ Convulxin was purified to homogeneity from the venom of Crotalus durissus terrificus. ${ }^{28}$ Other materials including fibrinogen were from sources indicated before. ${ }^{27}$ 


\section{Platelet and plasma preparation}

Blood was taken from healthy volunteers and from two patients with Glanzmann's thrombasthenia, with established deficiencies in integrin $\alpha_{111} \beta_{3},{ }^{29}$ after informed consent and in accordance with the Declaration of Helsinki. Approval was received from the local medical ethical committee. Blood was collected into 1/10 volume of 129 $\mathrm{mM}$ trisodium citrate. PRP was obtained by centrifuging at $240 \times \mathrm{g}$ for 15 minutes and platelet-free plasma (PFP) by centrifuging twice at 2,630 $\times g$ for 10 minutes. Platelet count in PRP was determined with a thrombocounter (Coulter Electronics) and normalised with autologous PFP. Citrate-anticoagulated PFP was partly defibrinated by a 10 minute treatment with low ancrod protease $(1.3 \mathrm{U} / \mathrm{mL})$. After centrifuging the fibrin clots that were formed, non-turbid plasma was isolated. The remaining fibrinogen content was determined at $\sim 0.5 \mathrm{mg} / \mathrm{mL}$ according to the conventional Clauss method based on turbidimetric measurements. ${ }^{30}$ When supplemented with platelets, the ancrod-treated plasma showed normal collagen-induced platelet aggregation responses.

For the preparation of washed platelets, blood was collected into $1 / 6$ volume of acid-citrate glucose solution (ACD, $80 \mathrm{mM}$ trisodium citrate, $52 \mathrm{mM}$ citric acid and 180 $\mathrm{mM}$ glucose). Platelets were obtained by centrifugation, washed in the presence of apyrase (0.1 U/mL ADPase), and resuspended in Hepes buffer $\mathrm{pH} 7.45$ (10 mM Hepes, $136 \mathrm{mM} \mathrm{NaCl}, 2.7 \mathrm{mM} \mathrm{KCl}, 2 \mathrm{mM} \mathrm{MgCl}, 0.1 \%$ glucose and $0.1 \% \mathrm{BSA}$ ) at a count of 1.0 $\times 10^{8} / \mathrm{mL}^{27}$

For experiments with reconstituted PRP, partly defibrinated plasma was supplemented with washed platelets $\left(1.0 \times 10^{8} / \mathrm{mL}\right)$. Apyrase was not added, because of the presence of autologous exonucleotidase activity in plasma.

\section{Flow cytometry}

Washed, unstirred platelets in Hepes buffer were activated with PAR1 agonist SFLLRN $(15 \mu \mathrm{M})$ or thrombin $(10 \mathrm{nM})$ in combination with convulxin $(50 \mathrm{ng} / \mathrm{mL})$. Alternatively, the washed platelets were resuspended in ancrod-treated citrate plasma at $1.0 \times 10^{8} \mathrm{I}$ $\mathrm{mL}$. The reconstituted PRP was activated with tissue factor (2 pM) and $\mathrm{CaCl}_{2}(16.6$ $\mathrm{mM}$ ) at $37^{\circ} \mathrm{C}$. After 15 minutes of activation, PS exposure and integrin activation were determined with FITC-labelled annexin A5 or FITC-labelled mAb against plateletbound human fibrinogen, respectively, using flow cytometry. ${ }^{12}$ For cytosolic $\mathrm{Ca}^{2+}$ measurements, platelets were loaded with Fura-Red $(22 \mu \mathrm{M})$ and pluronic $(400 \mu \mathrm{g} /$ $\mathrm{mL})$ in the presence of apyrase $(0.1 \mathrm{U} / \mathrm{mL}$ ADPase). After a washing step, the loaded platelets were resuspended in ancrod-treated citrate plasma, which was triggered by tissue factor (2 pM) and $\mathrm{CaCl}_{2}(16.6 \mathrm{mM})$ at $37^{\circ} \mathrm{C}$. Increases in cytosolic $\mathrm{Ca}^{2+}$, apparent as decreases in fluorescence, were recorded in time by flow cytometry. ${ }^{26}$ 


\section{Thrombin generation}

Thrombin generation was determined in normalised PRP $\left(1.5 \times 10^{8}\right.$ platelets $\left./ \mathrm{mL}\right)$ or, as a control, in PFP supplemented with phospholipid vesicles $(10 \mu \mathrm{M})$. The normalised PRP from control subjects or a Glanzmann patient was activated with tissue factor/ $\mathrm{CaCl}_{2}$, and fluorescence accumulation was measured according to the thrombogram method under non-stirred conditions in a Fluoroskan Ascent well-plate reader at $37{ }^{\circ} \mathrm{C}^{13}$ Nanomolar thrombin concentrations were obtained by comparison with a human thrombin standard using Thrombinoscope software.

\section{Spectrofluorometry}

Platelets were loaded with Fura-2 when rises in cytosolic $\mathrm{Ca}^{2+}$ concentration were determined in the absence of plasma. ${ }^{31}$ Fura-2-loaded platelets were activated in the presence of $2 \mathrm{mM} \mathrm{CaCl}_{2}$ at slow stirring (100 rpm, $37^{\circ} \mathrm{C}$ ); inhibitors were given before (10 minutes) or after agonist addition, as indicated. Nanomolar changes in $\mathrm{Ca}^{2+}$ level were obtained by calibration procedures, described in detail elsewhere. ${ }^{32}$

\section{Fluorescence microscopy}

Glass coverslips were coated with $25 \mu \mathrm{L}$ of fibrinogen solution ( $1 \mathrm{mg} / \mathrm{mL})$, rinsed twice with saline, and incubated with washed (Fura-2-loaded) platelets in Hepes buffer $\mathrm{pH}$ $7.45\left(1.0 \times 10^{8} / \mathrm{mL}\right) .{ }^{32}$ Where indicated, the coverslips were coated with a low fibrinogen solution $(10 \mu \mathrm{g} / \mathrm{mL})$. After 30 minutes, non-adherent platelets were removed, and the adhered spreading platelets were stimulated with thrombin (10 $\mathrm{nM})$ in the presence of $2 \mathrm{mM} \mathrm{CaCl}_{2}$. Microscopic phase-contrast and fluorescence images of PS exposure (FITC-labelled annexin A5) were taken using a dual camera imaging system, controlled by Visitech software. ${ }^{33}$ Fluorescence ratio images of Fura-2 fluorescence were taken to obtain rises in $\left[\mathrm{Ca}^{2+}\right]_{\text {. }}$. For calibration, fluorescence values were obtained from $\mathrm{Ca}^{2+}{ }_{-}$ saturated and $\mathrm{Ca}^{2+}$-free lysed platelets containing the fluorescent probe, using the microscopic and camera settings as described. ${ }^{34}$

\section{Platelet isolation from coagulating plasma}

Washed platelets $\left(5 \times 10^{8}\right.$ platelets $/ \mathrm{mL}$ ) were reconstituted in ancrod-defibrinated plasma in the presence of GPRP $(1 \mathrm{mg} / \mathrm{mL})$ and cangrelor $(20 \mu \mathrm{M})$. Samples of reconstituted PRP were preincubated with vehicle, Syk inhibitor II $(5 \mu \mathrm{M})$, and tirofiban $(10 \mu \mathrm{g} / \mathrm{mL})$, as indicated, and activated with tissue factor (2 pM) and $\mathrm{CaCl}_{2}$ $(16.6 \mathrm{mM})$. Initial attempts were made to isolate platelets from the activated PRP by centrifugation or gel filtration, but these were unsuccessful. Hence, a novel method was developed, in which platelets were captured from activated PRP by addition of anti-CD31 mAb-coated magnetic microbeads. After 15 minutes of activation, these platelets were isolated by passage of the PRP through a separation column, and an 
immediate rinse to remove all plasma proteins. Isolated platelets in the separation column were immediately lysed by flowing with lysis buffer $(600 \mathrm{mM} \mathrm{NaCl}, 40$ $\mathrm{mM}$ Tris, 4 mM EGTA, $4 \mathrm{mM}$ EDTA, 4 \% nonidet-P40, $10 \mathrm{mM} \mathrm{Na} \mathrm{VO}_{4}, 4 \mathrm{mM}$ PMSF, $20 \mu \mathrm{g} / \mathrm{mL}$ leupeptin, $20 \mu \mathrm{g} / \mathrm{mL}$ aprotinin, $5 \mu \mathrm{g} / \mathrm{mL}$ pepstatin A). Lysates were frozen at $-80^{\circ} \mathrm{C}$ until use.

\section{Protein separation and western blotting}

Platelet lysates were separated by polyacrylamide gel electrophoresis and subjected to standard western blotting. Blots were stained for phosphorylated Syk with antiSyk Tyr ${ }^{525 / 526}$ mAb (1:1,000) and secondary HRP-conjugated secondary Ab (1:500). Total Syk was determined by reprobing with anti-Syk mAb $(1: 1,000)$ and HRP-conjugated secondary $A b(1: 1,000)$. To control for total platelet proteins, parallel blots were probed for $\alpha$-tubulin $(1: 1,000)$. Antibody staining was quantified by densitometric analysis. ${ }^{35}$

\section{Statistics}

Data are given as mean \pm standard error of the mean (SEM). Significance of differences was determined with the Mann-Whitney $U$ test or the independent samples $t$ test, as appropriate, using the statistical package for social sciences (SPSS 15.0).

\section{Results}

Roles of integrin $\alpha_{l l b} b_{3}$ in tissue factor-stimulated thrombin generation and platelet activation

Early reports suggest that various integrin $\alpha_{11 b} \beta_{3}$ antagonists differently affect thrombin generation in platelet-rich plasma (PRP). ${ }^{19,}{ }^{23}$ To verify this, we prepared human PRP and determined the effects on tissue factor-induced thrombin generation of three $\alpha_{11 b} \beta_{3}$ blockers, all in clinical use: the human/mouse chimeric monoclonal antibody fragment abciximab (blocking integrins $\alpha_{11 b} \beta_{3}$ and $\alpha_{v} \beta_{3}$ ), the peptide mimetic eptifibatide, and the non-peptide sulfonamido compound, tirofiban. When added to recalcified PRP, tirofiban dose-dependently suppressed and delayed thrombin generation induced by tissue factor (Fig. $1 A, B$ ). A similar reduction in thrombin generation was seen with the other $\alpha_{11 b} \beta_{3}$ blockers (Supplemental Fig. $1 A, B$ ). Tirofiban, abciximab and eptifibatide reduced the thrombin peak with $60 \%$ at $\mathrm{IC}_{50}$ values of $0.1,2$ and $1 \mu \mathrm{g} / \mathrm{mL}$, respectively. In spite of this reduction, a thrombin peak of $20 \mathrm{nM}$ was still reached, which is sufficiently high for maximal thrombin-induced platelet activation. Control experiments indicated that the integrin blockers were similarly effective in suppressing platelet aggregation of PPACK-anticoagulated PRP, stimulated with the PAR1 agonist SFLLRN (not shown). In contrast, none of the $\alpha_{11 b} \beta_{3}$ blockers affected 
A

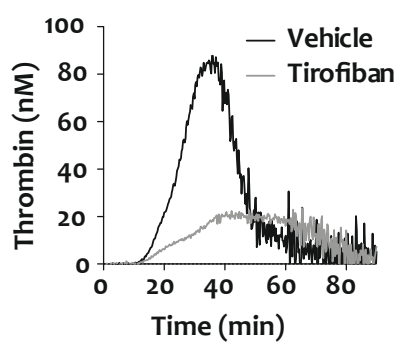

B

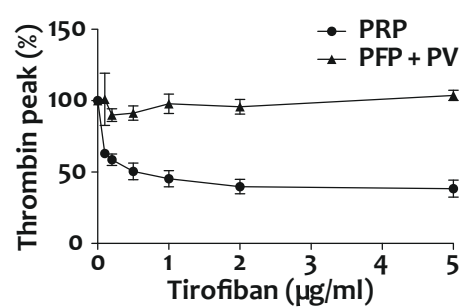

C

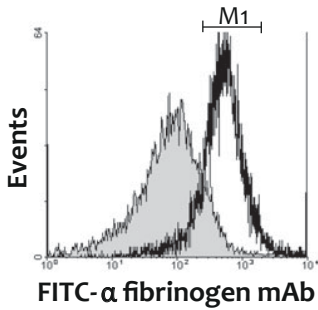

$\mathrm{E}$

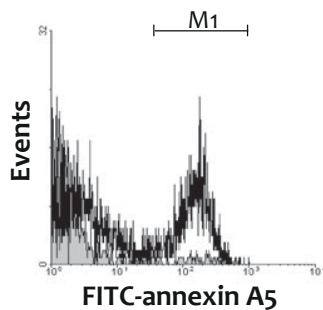

D

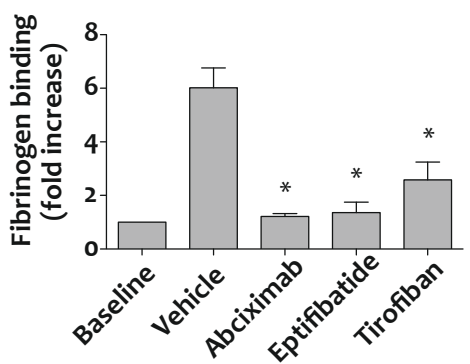

$\mathrm{F}$

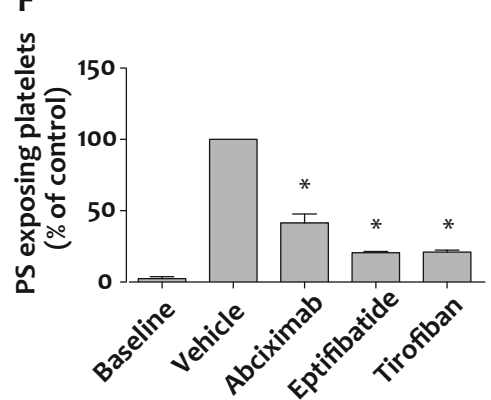

Figure 1. Blocking of integrin $\alpha_{11 b} \beta_{3}$ suppresses PS exposure and thrombin generation in tissue factorstimulated PRP. (A, B) PRP $\left(1.0 \times 10^{8}\right.$ platelets $\left./ \mathrm{mL}\right)$ or PFP supplemented with phospholipid vesicles (PV, $10 \mu \mathrm{M}$ ) was preincubated with vehicle or tirofiban $(0.1-5 \mu \mathrm{g} / \mathrm{mL})$ for 20 minutes. Thrombin generation was stimulated with tissue factor ( $1 \mathrm{pM}$ ) and $\mathrm{CaCl}_{2}$. (A) Representative thrombin generation curves with PRP, (B) dose-dependent inhibition of thrombin generation only in the presence of platelets. Mean \pm SEM $(n=3-7)$. (C-F) Ancrod-treated PRP was preincubated with vehicle, abciximab (10 $\mu \mathrm{g} / \mathrm{mL})$, Eptifibatide $(10 \mu \mathrm{g} / \mathrm{mL})$ or tirofiban $(1 \mu \mathrm{g} / \mathrm{mL})$, and thrombin generation was stimulated as above. After 15 minutes, platelet activation was evaluated by flow cytometry. (C, D) Platelet fibrin(ogen) binding measured with FITC-labelled anti-fibrinogen $\mathrm{mAb}$. Data are fold increase in fluorescence relative to baseline (prior to activation). (E, F) Platelet PS exposure measured with FITC-annexin A5. Data are fractions of PS-exposing platelets (compared to vehicle control). $M 1$ indicates platelet populations with increased fluorescence. Means $\pm \operatorname{SEM}(n=3-6) ;{ }^{*} p<0.05$ versus vehicle.

thrombin generation in plasma containing phospholipids instead of platelets (Fig. 1B and Supplemental Fig. 1B), thus demonstrating that the blocker effects required the presence of platelets.

In order to study tissue factor-induced platelet activation in plasma, formation of disturbing fibrin clots needed to be prevented. Therefore, human plasma was partly defibrinated with a low concentration $(1.3 \mathrm{U} / \mathrm{mL})$ of the snake venom ancrod, which induces fibrin clotting without thrombin generation..$^{36}$ After removal of the ancrod clots by centrifugation, the remaining plasma contained a residual concentration of $\sim 0.5 \mathrm{mg} / \mathrm{mL}$ fibrinogen, which does not form large clots. In this plasma reconstituted with platelets, $\alpha_{1 \mathrm{lb}} \beta_{3}$ blockers suppressed tissue factor-induced thrombin generation with $40-50 \%$ (data not shown). Flow cytometry demonstrated that, after tissue factor stimulation, fibrin(ogen) binding to platelets was antagonised by any of the three 
$\alpha_{11 b} \beta_{3}$ blockers (Fig. 1C, D). Furthermore, tissue factor induced PS exposure in $35 \%$ of the platelets, as determined by staining with FITC-annexin A5 (Fig. 1E). In the absence of tissue factor, fibrin(ogen) binding and PS exposure were quite low. Strikingly, blockage of $\alpha_{11 b} \beta_{3}$ greatly reduced the tissue factor-induced PS exposure with $60 \%$ (abciximab) or $80 \%$ (eptifibatide, tirofiban) at maximally effective concentrations (Fig. 1F). Flow cytometry furthermore indicated that the integrin blockers suppressed formation of PS-exposing microparticles by $>50 \%$, as reported before. ${ }^{17}$ Similar experiments were performed in the presence of apyrase $(0.1 \mathrm{U} / \mathrm{mL})$, in which case again abciximab, eptifibatide, and tirofiban reduced the number of PS-exposing platelets by $75-85 \%$.

A frequently used way of provoking PS exposure is by stimulating washed platelets with thrombin in combination with collagen receptor agonist, convulxin. ${ }^{37}$ Considering that these platelets secrete fibrinogen which binds to $\alpha_{11 b} \beta_{3}$ in an autocrine way, we investigated whether in this condition $\alpha_{11} \beta_{3}$ blocking may also influence PS exposure. Flow cytometry indicated that the co-stimulation of isolated platelets with thrombin $(10 \mathrm{nM})$ and convulxin $(50 \mathrm{ng} / \mathrm{mL})$ resulted in large fractions of platelets binding fibrin(ogen) and exposing PS (Fig. 2A). Dose-response experiments indicated that this concentration of thrombin (10 $\mathrm{nM}$ ) was maximally effective (not shown), similarly as described before. ${ }^{38}$ All integrin blockers caused a substantial decrease
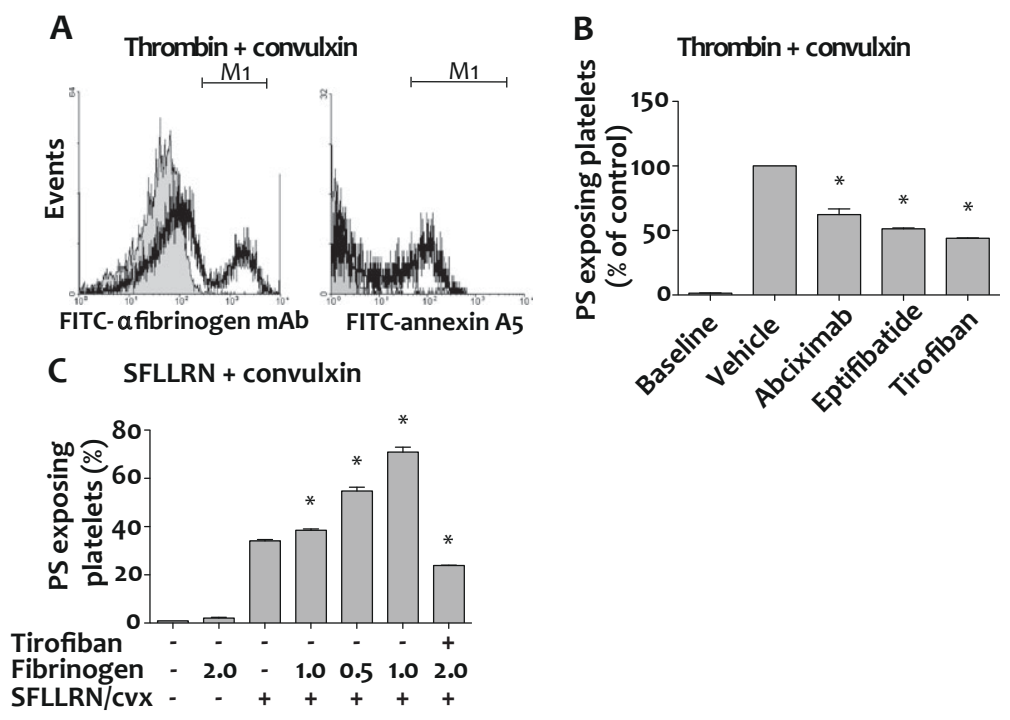

Figure 2. Blocking of integrin $\alpha_{11 \mathrm{~b}} \beta_{3}$ suppresses PS exposure of convulxin/thrombin-stimulated platelets. Washed platelets containing apyrase were activated with thrombin (10 nM) or SFLLRN (15 $\mu M)$ plus convulxin $(50 \mathrm{ng} / \mathrm{mL})$, as indicated, in the presence of $\mathrm{CaCl}_{2}(2 \mathrm{mM})$ for 15 minutes. Pretreatment with abciximab $(10 \mu \mathrm{g} / \mathrm{mL})$, eptifibatide $(10 \mu \mathrm{g} / \mathrm{mL})$ or tirofiban $(1 \mu \mathrm{g} / \mathrm{mL})$. (A) Histograms of fibrin(ogen) binding (FITC-anti-fibrinogen mAb) and PS exposure (FITC-annexin A5). (B) Quantitative effect of $\alpha_{11 b} \beta_{3}$ blockage on platelet PS exposure. (C) Effect of added human fibrinogen (0.1-2.0 mg/mL) on PS exposure. Means $\pm \operatorname{SEM}(n=3) ;{ }^{*} p<0.05$ versus vehicle. 
in PS exposure of $35 \%$ (abciximab) or $50 \%$ (eptifibatide, tirofiban) of the control condition (Fig. 2B). This suggested a supportive role of $\alpha_{11 b} \beta_{3}$ in thrombin and collagen receptor-induced PS exposure via interaction with (secreted) fibrinogen.

To further study this under conditions where fibrin clot formation was prevented, the platelets were activated with convulxin plus PAR1 agonist SFLLRN. Addition of exogenous human fibrinogen resulted in a dose-dependent stimulatory effect on PS exposure, increasing the fraction of PS-exposing platelets from $35 \%$ up to $70 \%$ (Fig. 2C). In contrast, addition of fibrinogen alone, without other agonists, did not stimulate PS exposure. Pretreatment with tirofiban (Fig. 2C) or other integrin blockers (not shown) completely reversed the stimulating effect of fibrinogen. Comparable results were obtained with bovine and human fibrinogen (not shown). Together, these results point to a role of integrin $\alpha_{11 b} \beta_{3}$, likely via interaction with fibrin(ogen) on platelet PS exposure both in tissue factor-stimulated PRP (resulting in increased thrombin generation), and in washed platelets stimulated with thrombin and collagen receptor agonists.
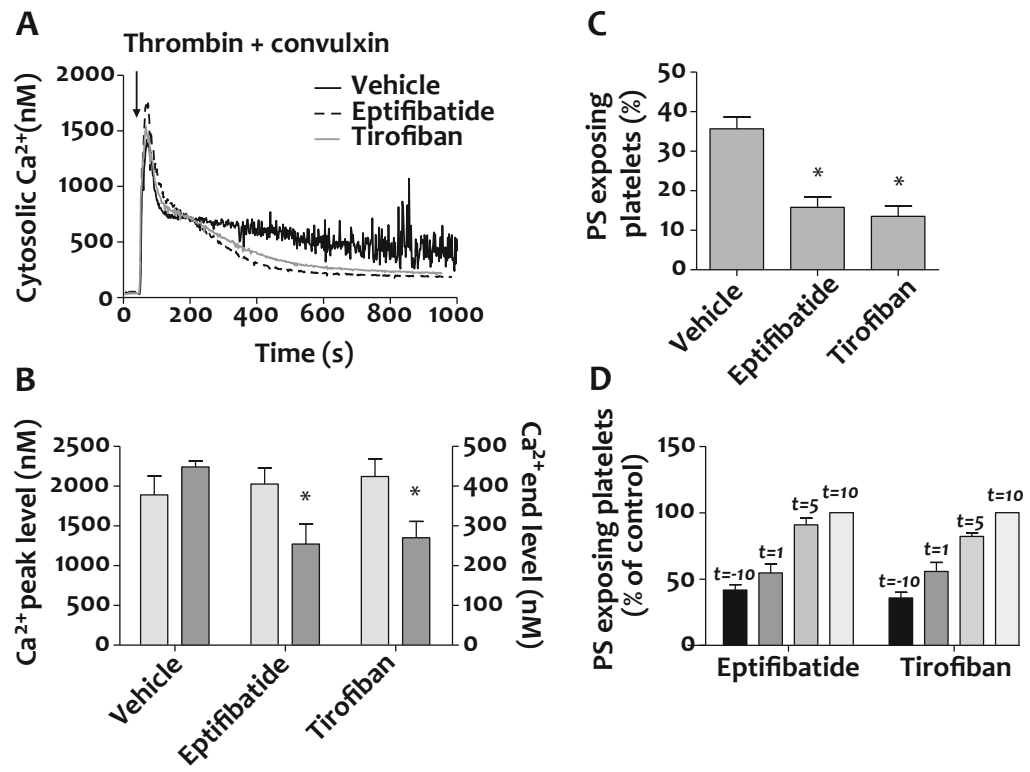

Figure 3. Blocking of $\alpha_{11 b} \beta_{3}$ suppresses long-term platelet $\mathrm{Ca}^{2+}$ responses and PS exposure. Fura-2-loaded platelets containing apyrase were preincubated with vehicle (control), eptifibatide $(10 \mu \mathrm{g} / \mathrm{mL}$ ) or tirofiban $(1 \mu \mathrm{g} / \mathrm{mL})$. Cells were then activated with thrombin $(10 \mathrm{nM})$ plus convulxin $(50 \mathrm{ng} / \mathrm{mL})$ and $1 \mathrm{mM} \mathrm{CaCl}$. (A) Representative platelet $\mathrm{Ca}^{2+}$ responses. (B) Effect of integrin blockers on $\mathrm{Ca}^{2+}$ peak levels (light grey) and 15-minute end levels (dark grey). (C) Effect of blockers on fractions of PS-exposing platelets after 15 minutes, analysed by flow cytometry. (D) Effect of addition of eptifibatide or tirofiban at different time points before $(t=-10$ minutes $)$ or after $(t=1-10$ minutes $)$ activation. Fractions of PS-exposing platelets after 15 minutes. Data are relative to control condition without integrin blocker. Means \pm SEM $(n=3-5)$; ${ }^{*} p<0.05$ versus vehicle. 
Signalling role of integrin $\alpha_{I I b} b_{3}$ in $\mathrm{Ca}^{2+}$ and procoagulant platelet responses

Others have suggested that inhibitory effects of $\alpha_{11 b} \beta_{3}$ blockers on platelet PS exposure may occur independently of modulating $\mathrm{Ca}^{2+}$ responses. ${ }^{22,39} \mathrm{Also}$, in stored platelets, PS exposure can occur independently of elevated $\mathrm{Ca}^{2+} .^{40}$ We therefore re-examined a role of $\alpha_{11 \mathrm{~b}} \beta_{3}$ in $\mathrm{Ca}^{2+}$-signalling by stimulating washed suspensions of Fura-2-loaded platelets with thrombin and convulxin. While measuring rises in $\mathrm{Ca}^{2+}$, samples were taken for flow cytometric determination of PS exposure. The agonists caused a potent increase in $\mathrm{Ca}^{2+}$ peak, which was followed by a sustained elevated level (450 nM), persisting during 15 minutes (Fig. 3A). Pretreatment with eptifibatide or tirofiban did not influence the initial $\mathrm{Ca}^{2+}$ peak, but it markedly reduced the sustained high $\mathrm{Ca}^{2+}$ level to $56 \pm 10 \%$ or $60 \pm 8 \%$ of the control value, respectively (Fig. $3 \mathrm{~A}, \mathrm{~B}$ ). The reduction in sustained $\mathrm{Ca}^{2+}$ response was accompanied by a proportional decrease in PS exposure from $36 \pm 3 \%$ to $16 \pm 3 \%$ or $14 \pm 3 \%$, respectively (Fig. 3 C). Reasoning that persistent integrin signalling may prolong these $\mathrm{Ca}^{2+}$ responses and then contribute to PS exposure, we added the integrin blockers at various time points after thrombin/convulxin. Addition of eptifibatide or tirofiban at 1 minute after activation still caused a substantial reduction in the fraction of PS-exposing platelets, whereas addition after 5-10 minutes resulted in progressively less inhibition (Fig. 3D).

A Glanzmann patient 1

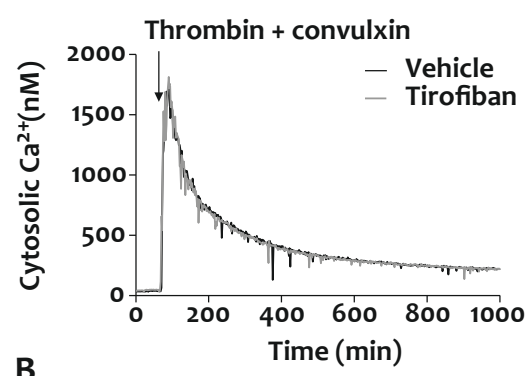

C

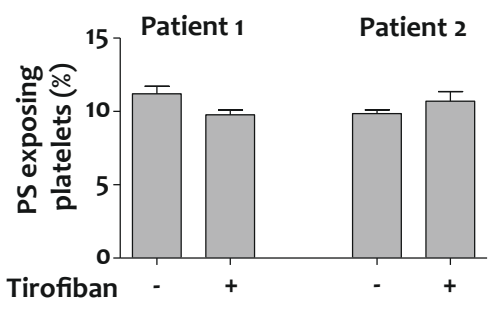

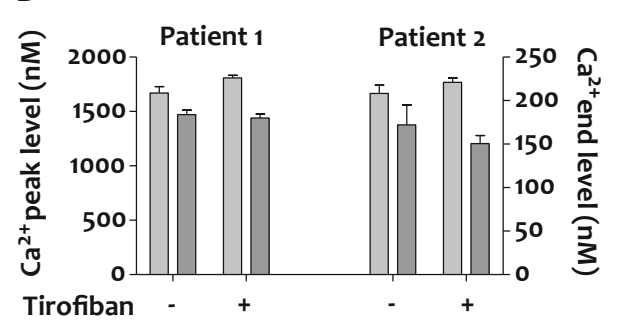

Figure 4. Impaired long-term $\mathrm{Ca}^{2+}$ responses and PS exposure in activated Glanzmann platelets. Suspensions of Fura-2-loaded platelets containing apyrase from two Glanzmann patients $(1,2)$ were pretreated with vehicle or tirofiban $(1 \mu \mathrm{g} / \mathrm{mL})$. Platelets were then activated with thrombin $(10 \mathrm{nM})$ plus convulxin $(50 \mathrm{ng} / \mathrm{mL})$ in the presence of $1 \mathrm{mM} \mathrm{CaCl}$. (A) Representative traces of $\mathrm{Ca}^{2+}$ responses. (B) Averaged $\mathrm{Ca}^{2+}$ peak levels (light grey) and 15-minute end levels (dark grey). (C) Fractions of PS-exposing platelets after 15 minutes, showing no effect of tirofiban. Means $\pm \operatorname{SEM}(n=3$ experiments). 
To further confirm the contribution of integrin signalling in PS exposure, platelets were obtained from two Glanzmann patients with complete deficiency in $\mathrm{a}_{11 \mathrm{~b}} \beta_{3}$ expression. Loaded with Fura-2, the platelets showed high peak rises in $\mathrm{Ca}^{2+}$ in response to thrombin/convulxin, but at later time points $\mathrm{Ca}^{2+}$ levels declined to $\sim 150$ nM (Fig. 4A, B). This corresponded to a low amount of 10-12 \% PS-exposing platelets (Fig. 4C). Addition of tirofiban altered neither the late $\mathrm{Ca}^{2+}$ response nor the low exposure of PS. Collectively, these data point to a role of $\alpha_{11 \mathrm{~b}} \beta_{3}$-mediated signalling in long-term $\mathrm{Ca}^{2+}$ rises induced by thrombin and collagen receptor agonists and thereby in development of platelet procoagulant activity.

Signalling role of integrin $\alpha_{\| 1} b_{3}$ in $\mathrm{Ca}^{2+}$ and procoagulant responses of platelets spreading on fibrinogen

Outside-in signalling by integrin $\alpha_{11 \mathrm{~b}} \beta_{3}$ mediates the spreading of platelets on fibrinogen surfaces. ${ }^{5}$ In Fura-2-loaded platelets, we found that spreading was accompanied by irregular, low-amplitude $\mathrm{Ca}^{2+}$ spikes (Fig. $5 \mathrm{~A}$ ). In most of these platelets, thrombin
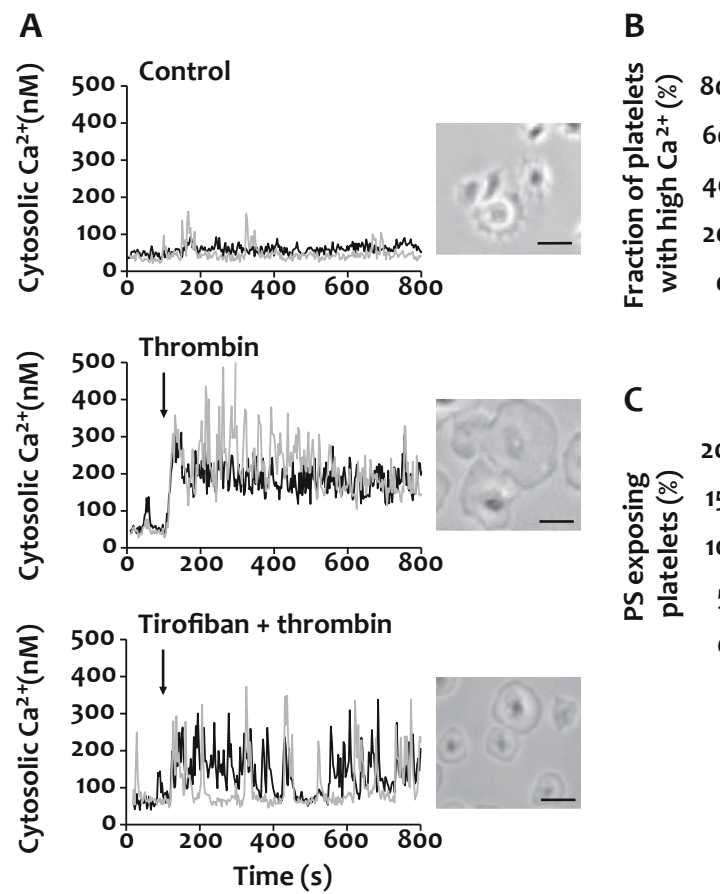

C
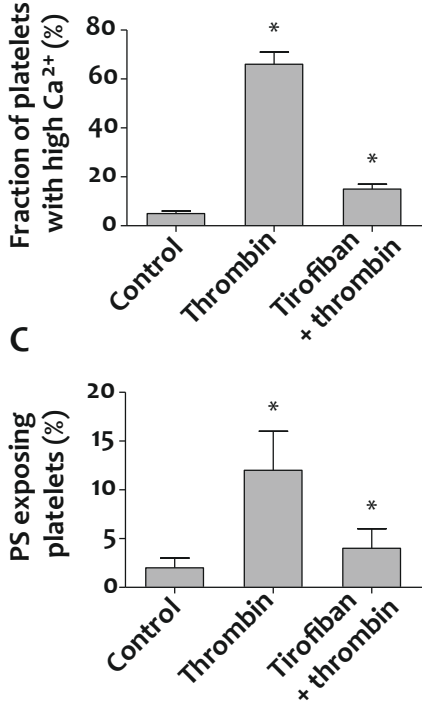

Figure 5. Blocking of integrin $\alpha_{11 b} \beta_{3}$ reduces $\mathrm{Ca}^{2+}$ responses and PS exposure of spreading platelets. Fura-2-loaded platelets were adhered to coated fibrinogen in the absence (control) or presence of tirofiban $\left(1 \mu \mathrm{g} / \mathrm{mL}\right.$ ) for 30 minutes. Rises in $\mathrm{Ca}^{2+}$ in single, adhered platelets in response to thrombin (10 $\mathrm{nM}$ ) measured by fluorescence ratio imaging. (A) Traces of $\mathrm{Ca}^{2+}$ rises of two representative platelets; also brightfield contrast images after 10 minutes (bars, $10 \mu \mathrm{m}$ ). (B) Fractions of platelets with elevated $\mathrm{Ca}^{2+}$ (>1.2-fold signal) after 10 minutes. (C) Fractions of platelets binding FITC-annexin A5 after 10 minutes. Means $\pm \operatorname{SEM}(n=4-6) ;{ }^{*} p<0.05$ versus control. 
induced a persistently high $\mathrm{Ca}^{2+}$ level and stimulated the spreading process (Fig. 5A, $B$ ). To investigate a role of integrin signalling, the platelets were preincubated with tirofiban prior to thrombin stimulation. This retarded the spreading process, and suppressed the $\mathrm{Ca}^{2+}$ response, in a way that the persistent $\mathrm{Ca}^{2+}$ rise changed into a pattern of repetitive $\mathrm{Ca}^{2+}$ spiking. Furthermore, tirofiban reduced the fractions of PS-exposing platelets with thrombin from $15 \%$ to only $2.5 \%$ (Fig. 5 C). The inhibiting effects of tirofiban were preserved on a surface coated with a low fibrinogen solution of $10 \mu \mathrm{g} / \mathrm{mL}$ (not shown). Thrombin stimulation of adhered, non-spread platelets (5 minutes fibrinogen adhesion) similarly resulted in a low fraction of PS-exposing platelets of $2.5 \pm 0.4 \%$. Control experiments were carried out with DM-BAPTA-loaded platelets, where basal levels of $\mathrm{Ca}^{2+}$ amounted $\sim 20 \mathrm{nM}$, and thrombin addition resulted in neither $\mathrm{Ca}^{2+}$ rises nor PS exposure (data not shown).

To further verify a role of $\alpha_{11 b} \beta_{3}$ in thrombin-induced responses of fibrinogenadhered platelets, similar experiments were performed with the platelets from two Glanzmann patients. While these platelets hardly spread on fibrinogen, they also remained low in PS exposure with only $\sim 2 \%$ annexin A5 binding after thrombin stimulation (Supplemental Fig. 2). These data thus suggest that integrin outsidein signalling during platelet spreading stimulates thrombin-induced procoagulant activity.

Contribution of integrin $\alpha_{11 b} b_{3}$ to platelet procoagulant response via Syk kinase activation Integrin $\alpha_{11 b} \beta_{3}$-mediated outside-in signalling triggers inactivation of RhoA and
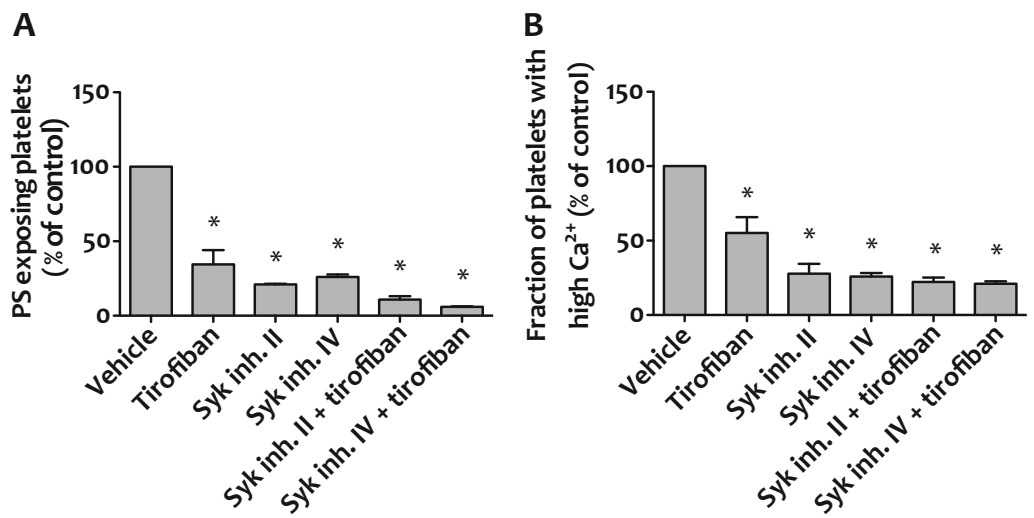

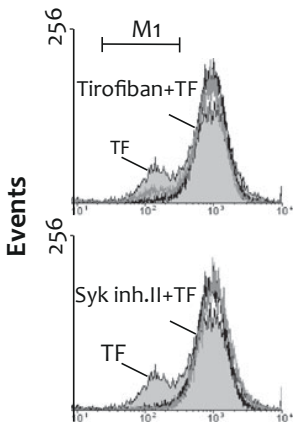

Fura-Red

Figure 6. Contribution of Syk kinase to platelet activation in tissue factor-stimulated plasma. Fura-Red loaded platelets in ancrod-treated plasma $\left(1.0 \times 10^{8} / \mathrm{mL}\right)$ were preincubated with vehicle, Syk inhibitor II or IV $(10 \mu \mathrm{M})$, and/or tirofiban $(1 \mu \mathrm{g} / \mathrm{mL})$, as described for Fig. 1. Cangrelor (AR-C, $10 \mu \mathrm{M})$ was present to eliminate $\mathrm{P}_{2} \mathrm{Y}_{12}$-dependent signalling events. PRP was then stimulated with $1 \mathrm{pM}$ tissue factor and $\mathrm{CaCl}_{2}$ for 15 minutes. (A) Fractions of PS-exposing platelets determined by FITC-annexin A5 binding. (B) Fractions of platelets with high $\mathrm{Ca}^{2+}$ as determined by flow cytometry $(\mathrm{M} 1)$. Flow cytometric histograms of FuraRed fluorescence. Note that decreased Fura-Red fluorescence points to high $\mathrm{Ca}^{2+}(\mathrm{M} 1)$. Histograms of unstimulated platelets (black), and tissue factor-stimulated platelets with vehicle (light grey), tirofiban or Syk inhibitor II (dark grey). Means \pm SEM $(n=4) ;{ }^{*} p<0.05$ versus vehicle. 
activation of the protein tyrosine kinase Syk, resulting in phospholipase $C_{2}$ activation. ${ }^{6,}{ }^{41}$ Employing several approaches, we investigated a role of Syk in $\alpha_{11 b} \beta_{3-}$ dependent $\mathrm{Ca}^{2+}$ rises and procoagulant activity. First, we used the pharmacologic blockers, Syk inhibitor II and IV, which abolished collagen-induced aggregation of platelets in plasma at maximally effective concentrations of $10 \mu \mathrm{M}$. In tissue factorstimulated PRP, both inhibitors suppressed platelet PS exposure at a similar degree as tirofiban (Fig. 6A). Next, platelets were loaded with the $\mathrm{Ca}^{2+}$ probe Fura-Red, which allows the monitoring of $\mathrm{Ca}^{2+}$ rises in the presence of plasma by flow cytometry. ${ }^{42}$ Stimulation with tissue factor resulted in a prolonged rise in $\mathrm{Ca}^{2+}$ in the majority of platelets in plasma (observed as a decrease in Fura-Red fluorescence). This $\mathrm{Ca}^{2+}$ rise was reduced in the presence of tirofiban and even more so with Syk inhibitor II or IV (Fig. 6B).

A

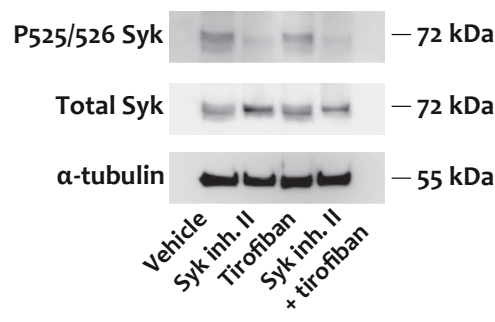

B

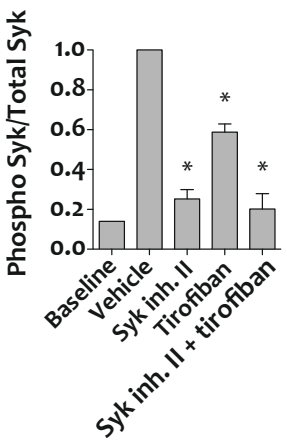

C

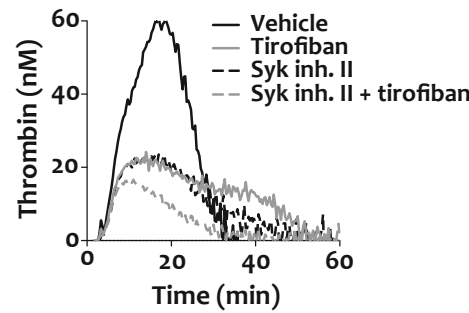

E

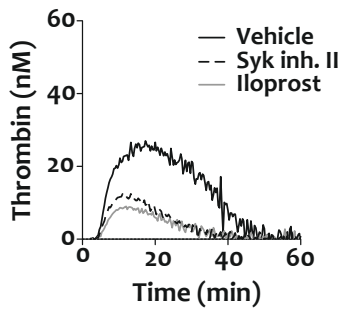

$\mathbf{F}$

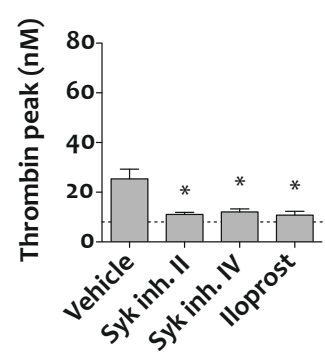

Figure 7. Contribution of integrin $\alpha_{11 b} \beta_{3}$ signalling and Syk kinase to thrombin generation in tissue factor-stimulated plasma. Reconstituted PRP was preincubated with vehicle, Syk inhibitor II (10 $\mu \mathrm{M})$, iloprost $(1 \mu \mathrm{M})$ and/or tirofiban $(1 \mu \mathrm{g} / \mathrm{mL})$, as indicated, and then stimulated with tissue factor $(1 \mathrm{pM})$ and $\mathrm{CaCl}_{2}$. Cangrelor (AR-C, $10 \mu \mathrm{M}$ ) was present to eliminate $\mathrm{P}_{2} \mathrm{Y}_{12}$-dependent signalling events. Control condition (baseline) was without tissue factor. (A, B) Effects of tirofiban and Syk inhibitor on Tyr $525 / 526$ phosphorylation of Syk. Platelets were isolated from activated plasma (15 minutes) using anti-CD31coupled magnetic beads and examined for protein phosphorylation. Representative western blots after probing for phospho-Syk (Tyr $\left.{ }^{525 / 526}\right)$, and reprobing for total Syk; parallel blots were stained for $a$-tubulin. Shown is the ratio of phospho-Syk/total Syk assessed by densitometric analysis $(n=6)$. (C-F) Effects of tirofiban and Syk inhibitor on tissue factor-induced thrombin generation in PRP. Blood was collected on citrate $(n=8)(C, D)$; or on citrate plus tirofiban $(1 \mu \mathrm{g} / \mathrm{mL})(n=3)(E, F)$. Representative thrombin generation curves and thrombin peak heights. Dotted lines in bar graphs indicate residual thrombin formed in plasma not containing platelets. Means \pm SEM; * $p<0.05$ versus vehicle. 
Third, we directly examined the activation of Syk in tissue factor-stimulated PRP. It appeared not to be possible to collect these platelets from plasma by centrifugation or gel filtration. Hence, we developed a method to isolate platelets after tissue factor stimulation by using magnetic beads coupled to anti-CD31 mAb. Lysates of the isolated platelets were subjected to gel electrophoresis and western blotting, and probed for phosphorylation of Syk at Tyr ${ }^{525 / 526}$, which is an essential step in Syk activation. ${ }^{43}$ While no Syk phosphorylation was detected in the absence of tissue factor, platelet stimulation with tissue factor stimulation markedly increased the phosphorylation, which event was completely prevented by Syk inhibitor II (Fig. 7A, B). Pretreatment with tirofiban substantially but not completely antagonised $\mathrm{Tyr} \mathrm{r}^{52 / 526}$ phosphorylation, suggesting a prominent role of $\alpha_{11 b} \beta_{3}$-dependent signalling to Syk in the tissue factoractivated PRP.

Fourth, we directly investigated the effects of Syk inhibitors II and IV on tissue factor-induced thrombin generation. Using citrate-anticoagulated PRP, it appeared that both compounds were similarly effective as tirofiban in the suppression of thrombin generation, while there was no additional effect of a combination with tirofiban (Fig. 7C, D). As platelet-dependent thrombin generation was still incompletely blocked, we considered the possibility that the platelets exhibited residual $\alpha_{11 b} \beta_{3}$ dependent signalling during blood collection and PRP preparation. To investigate this, blood was collected on citrate anticoagulant plus tirofiban. Indeed, with tirofiban initially present, tissue factor-induced thrombin generation was reduced, while either Syk inhibitor fully reduced the thrombin peak to the level obtained with the strong platelet inhibitor, iloprost (Fig. 7E, F). In fact, this residual, low thrombin generation was also present in plasma devoid of platelets, and could be ascribed to the presence of microparticles. Similar results were obtained with blood collected on Syk inhibitor II (not shown).

Finally, thrombin generation experiments were performed with PRP from a Glanzmann patient. In the patient PRP, tissue factor stimulation evoked limited thrombin generation, which, however, was not influenced by the presence of tirofiban (Supplemental Fig. 3). Similar to control PRP with tirofiban, addition of Syk inhibitor caused an additional decrease in thrombin generation. Taken together, these various sets of data show a substantial role of $\alpha_{\mathrm{Il}} \beta_{3}$ and Syk kinase in tissue factor-induced PS exposure and thrombin generation. Furthermore, they point to the existence of a pathway of thrombin generation that is dependent on Syk, but not on integrin activation.

\section{Discussion}

This paper reveals a new role of integrin $\alpha_{11 b} \beta_{3}$-dependent signalling via Syk kinase in tissue factor-induced platelet procoagulant activity and thrombin generation in plasma. The results point to a pathway where initial traces of thrombin triggered by tissue factor activate platelets to expose procoagulant PS, resulting in a cycle of 
thrombin generation and platelet activation that is greatly enforced and prolonged by integrin-dependent signalling to Syk activation and $\mathrm{Ca}^{2+}$ rises. The data furthermore identify a Syk-dependent, but integrin-independent pathway of thrombin generation.

In tissue factor-stimulated PRP, we found that the blockage of $\alpha_{11 b} \beta_{3}$ with different antagonists suppresses platelet $\mathrm{Ca}^{2+}$ responses, PS exposure and thrombin generation in a dose-dependent way. However, in washed platelets stimulated via thrombin and collagen receptors, integrin blockage also suppressed long-term $\mathrm{Ca}^{2+}$ rises along with PS exposure, while added fibrinogen enhanced these responses. Furthermore, during platelet spreading on fibrinogen, a process known to rely on $\alpha_{11 b} \beta_{3}$ outside-in signalling, blocking of the integrin resulted in $\mathrm{Ca}^{2+}$ signalling and PS exposure in response to maximally effective concentrations of thrombin. Confirmative evidence for a signalling role of $\alpha_{11 b} \beta_{3}$ came from the observation that long-term $\mathrm{Ca}^{2+}$ responses and PS exposure were reduced in platelets from two patients with Glanzmann's thrombasthenia, lacking $\alpha_{11 b} \beta_{3}$. Together, these data indicate that, in platelets stimulated with $\mathrm{Ca}^{2+}$ mobilising agonists, $\alpha_{\mathrm{Il}} \beta_{3}$ outside-in signalling prolongs the $\mathrm{Ca}^{2+}$ signal, increases procoagulant activity, and hence supports tissue factorstimulated thrombin generation on the platelet surface.

Anticoagulant effects of platelet $\alpha_{11 b} \beta_{3}$ antagonists have been reported by several authors, ${ }^{13,16,17}$ but the mechanism was not disclosed. Several groups reported that integrin blockers were unable to change platelet $\mathrm{Ca}^{2+}$ responses to collagen and/ or thrombin. ${ }^{22,23,39}$ However, the measurements mostly concerned initial $\mathrm{Ca}^{2+}$ rises, while in our hands only late $\mathrm{Ca}^{2+}$ signals appear to be affected. Interestingly, one study does describe long-term inhibition of collagen/thrombin-induced $\mathrm{Ca}^{2+}$ responses with abciximab but not with other integrin blockers specifically under conditions of stirring. ${ }^{23}$ This contrasts with the present findings where appropriate concentrations of different integrin blockers all had similar effects. Another published finding that $\alpha_{11 \mathrm{~b}} \beta_{3}$ blockage reduces shear-dependent $\mathrm{Ca}^{2+}$ responses and microparticle release ${ }^{19}$, ${ }^{44}$ can be explained by increased fibrinogen secretion of platelets subjected to a high shear rate.

The (patho)physiological relevance of this work comes from recent data that $\beta_{3}$-mutated mice with a deficiency in platelet outside-in signalling and tyrosine phosphorylation are protected from arterial thrombus formation after carotid artery injury with $\mathrm{FeCl}_{3}, 45$ i.e. a mouse thrombosis model where thrombus formation depends on tissue factor activity and on procoagulant, PS-exposing platelets. ${ }^{12,46-48}$

Platelets from mice deficient in phospholipase $\mathrm{C}_{2}$ have shown reduced $\mathrm{Ca}^{2+}$ signals and spreading on immobilised fibrinogen. ${ }^{44,49}$ Based on these and other data a scheme has been proposed of $\alpha_{\| l b} \beta_{3}$-induced signalling via Src and Syk kinases to activation of phospholipase $C \gamma 2 .{ }^{6}$ This signalling scheme was confirmed by recent proteomic analyses demonstrating the presence of many tyrosine phosphorylated proteins in human platelets spread on fibrinogen, among which multiple tyrosine kinases. ${ }^{4}$ The present finding points to a particular role of Syk phosphorylation and subsequent phospholipase $C_{\gamma 2}$ activation in $\alpha_{11 \mathrm{~b}} \beta_{3}$-dependent procoagulant activity 
and thrombin generation upon triggering with tissue factor. Evidence for this came from the reduction in Syk tyrosine phosphorylation by integrin blockage and by two Syk inhibitors. These experiments were carried out under conditions eliminating a contribution of $\mathrm{P}_{2} \mathrm{Y}_{12}$ signalling. ${ }^{24,50}$

Interestingly, in addition to a novel $\alpha_{11 b} \beta_{3}$-dependent role of Syk kinase in platelet procoagulant activity, our data also point to an $\alpha_{11 b} \beta_{3}$-independent role of Syk in this process. We have not yet unravelled the mechanism of $\alpha_{11 b} \beta_{3}$-independent Syk activation, but according to the literature this activation pathway can include FcyRIla, ${ }^{51}$ glycoprotein Ib-IX-V complex, ${ }^{52,53}$ or CLEC2, ${ }^{54}$ all of which have been shown to activate Syk. Altogether, our results point to a signalling scheme where fibrin(ogen)-induced integrin activation supports a Syk/phospholipase $C_{\gamma} 2$ pathway, resulting in prolonged $\mathrm{Ca}^{2+}$ and PS exposure and thrombin generation in plasma.

\section{Acknowledgments}

We thank Dr. S. Cauwenberghs for contribution in initial experiments. We acknowledge support from the Cardiovascular Centre, $\mathrm{MUMC}^{+}$, Maastricht, and the Landsteiner Foundation for Blood Transfusion Research, LSBR1006. Supported by the Thrombosis Expertise Center Maastricht, the Landsteiner Foundation for Transfusion Research and the Marie-Curie Early Stage Researcher Program 2005-020706-3.

\section{References}

1. Cosemans JM, Iserbyt BF, Deckmyn H, Heemskerk JW. Multiple ways to switch platelet integrins on and off. J Thromb Haemost. 2008;6:1253-1261.

2. Ma YQ, Qin J, Plow EF. Platelet integrin $\alpha_{11 b} \beta_{3}$ : activation mechanisms. J Thromb Haemost. 2007;5:1345-1352.

3. Nieswandt B, Moser M, Pleines I, Varga-Szabo D, Monkley S, Critchley D, Fässler R. Loss of talin1 in platelets abrogates integrin activation, platelet aggregation, and thrombus formation in vitro and in vivo. J Exp Med. 2007;204:3113-3118.

4. Senis YA, Antrobus R, Severin S, Parguina AF, Rosa I, Zitzmann N, Watson SP, Garcia A. Proteomic analysis of integrin $\alpha_{11 b} \beta_{3}$ outside-in signaling reveals Src-kinase-independent phosphorylation of Dok-1 and Dok-3 leading to SHIP-1 interactions. J Thromb Haemost. 2009;7:1718-1726.

5. Shattil SJ, Newman PJ. Integrins: dynamic scaffolds for adhesion and signaling in platelets. Blood. 2004;104:1606-1615.

6. Watson SP, Auger JM, McCarty OJ, Pearce AC. GPVI and integrin $\alpha_{11 b} \beta_{3}$ signaling in platelets. $J$ Thromb Haemost. 2005;3:1752-1762.

7. Cauwenberghs S, Feijge MA, Harper AG, Sage SO, Curvers J, Heemskerk JW. Shedding of procoagulant microparticles from unstimulated platelets by integrin-mediated destabilization of actin cytoskeleton. FEBS Lett. 2006;580:5313-5320.

8. Schoenwaelder SM, Yuan Y, Cooray P, Salem HH, Jackson SP. Calpain cleavage of focal adhesion proteins regulates the cytoskeletal attachment of integrin $\alpha_{11 b} \beta_{3}$ (platelet glycoprotein Ilb/IIla) and the cellular retraction of fibrin clots. J Biol Chem. 1997;272:1694-1702.

9. Shattil SJ, Kashiwagi H, Pampori N. Integrin signaling: the platelet paradigm. Blood. 1998;91:26452657.

10. Heemskerk JW, Kuijpers MJ, Munnix IC, Siljander PR. Platelet collagen receptors and coagulation. A 
characteristic platelet response as possible target for antithrombotic treatment. Trends Cardiovasc Med. 2005;15:86-92.

11. Monroe DM, Hoffman M, Roberts HR. Platelets and thrombin generation. Arterioscler Thromb Vasc Biol. 2002;22:1381-1389.

12. Munnix IC, Kuijpers MJ, Auger J, Thomassen CM, Panizzi P, Van Zandvoort MA, Rosing J, Bock PE, Watson SP, Heemskerk JW. Segregation of platelet aggregatory and procoagulant microdomains in thrombus formation: regulation by transient integrin activation. Arterioscler Thromb Vasc Biol. 2007;27:2484-2490.

13. Vanschoonbeek K, Feijge MA, Van Kampen RJ, Kenis H, Hemker HC, Giesen PL, Heemskerk JW. Initiating and potentiating role of platelets in tissue factor-induced thrombin generation in the presence of plasma: subject-dependent variation in thrombogram characteristics. J Thromb Haemost. 2004;2:476-484.

14. Castoldi E, Collins PW, Williamson PL, Bevers EM. Compound heterozygosity for 2 novel TMEM16F mutations in a patient with Scott syndrome. Blood. 2011;117:4399-4400.

15. Zwaal RF, Schroit AJ. Pathophysiologic implications of membrane phospholipid asymmetry in blood cells. Blood. 1997;89:1121-1132.

16. Ilveskero S, Lassila R. Abciximab inhibits procoagulant activity but not the release reaction upon collagen- or clot-adherent platelets. J Thromb Haemost. 2003;1:805-813.

17. Reverter JC, Béguin S, Kessels H, Kumar R, Hemker HC, Coller BS. Inhibition of platelet-mediated, tissue factor-induced thrombin generation by the mouse/human chimeric $7 \mathrm{E} 3$ antibody. Potential implications for the effect of C7E3 Fab treatment on acute thrombosis and "clinical restenosis". J Clin Invest. 1996;98:863-874.

18. Gemmell $\mathrm{CH}$, Sefton MV, Yeo EL. Platelet-derived microparticle formation involves glycoprotein IIb-IIIa. Inhibition by RGDS and a Glanzmann's thrombasthenia defect. J Biol Chem. 1993;268:1458614589.

19. Goto S, Tamura N, Li M, Handa M, Ikeda Y, Handa S, Ruggeri ZM. Different effects of various antiGPIIb-IIla agents on shear-induced platelet activation and expression of procoagulant activity. $J$ Thromb Haemost. 2003;1:2022-2030.

20. Byzova TV, Plow EF. Networking in the hemostatic system. Integrin $\alpha_{11 b} \beta_{3}$ binds prothrombin and influences its activation. J Biol Chem. 1997;272:27183-27188.

21. Furman MI, Krueger LA, Frelinger AL $3^{\frac{\text { rd }}{}}$, Barnard MR, Mascelli MA, Nakada MT, Michelson AD. GPIIbIIla antagonist-induced reduction in platelet surface factor V/Va binding and phosphatidylserine expression in whole blood. Thromb Haemost. 2000;84:492-498.

22. Pedicord DL, Thomas BE, Mousa SA, Dicker IB. Glycoprotein IIb/IIla receptor antagonists inhibit the development of platelet procoagulant activity. Thromb Res. 1998;90:247-258.

23. Lages B, Weiss HJ. Greater inhibition of platelet procoagulant activity by antibody-derived glycoprotein Ilb-IIla inhibitors than by peptide and peptidomimetic inhibitors. $\mathrm{Br} J$ Haematol. 2001;113:65-71.

24. Léon C, Ravanat C, Freund M, Cazenave JP, Gachet C. Differential involvement of the $P_{2} Y_{1}$ and $P_{2} Y_{12}$ receptors in platelet procoagulant activity. Arterioscler Thromb Vasc Biol. 2003;23:1941-1947.

25. Storey RF, Sanderson HM, White AE, May JA, Cameron KE, Heptinstall S. The central role of the P2T receptor in amplification of human platelet activation, aggregation, secretion and procoagulant activity. Br J Haematol. 2000;110:925-934.

26. Van der Meijden PE, Feijge MA, Giesen PL, Huijberts M, Van Raak LP, Heemskerk JW. Platelet $P_{2} Y_{12}$ receptors enhance signalling towards procoagulant activity and thrombin generation. A study with healthy subjects and patients at thrombotic risk. Thromb Haemost. 2005;93:1128-1136.

27. Van der Meijden PE, Munnix IC, Auger JM, Govers-Riemslag JW, Cosemans JM, Kuijpers MJ, Spronk HM, Watson SP, Renné T, Heemskerk JW. Dual role of collagen in factor XII-dependent thrombus formation. Blood. 2009;114:881-890. 
28. Siljander P, Farndale RW, Feijge MA, Comfurius P, Kos S, Bevers EM, Heemskerk JW. Platelet adhesion enhances the glycoprotein VI-dependent procoagulant response: Involvement of p38 MAP kinase and calpain. Arterioscler Thromb Vasc Biol. 2001;21:618-627.

29. Rosado JA, Meijer EM, Hamulyák K, Novakova I, Heemskerk JW, Sage SO. Fibrinogen binding to the integrin $\alpha_{11 b} \beta_{3}$ modulates store-mediated calcium entry in human platelets. Blood. 2001;97:26482656.

30. Clauss A. Rapid physiological coagulation method in determination of fibrinogen. Acta Haematol. 1957;17:237-246.

31. Feijge MA, Van Pampus EC, Lacabaratz-Porret C, Hamulyák K, Levy-Toledano S, Enouf J, Heemskerk JW. Inter-individual variability in $\mathrm{Ca}^{2+}$ signalling in platelets from healthy volunteers: effects of aspirin and relationship with expression of endomembrane $\mathrm{Ca}^{2+}$-ATPases. $\mathrm{Br} J$ Haematol. 1998;102:850-859.

32. Heemskerk JW, Vuist WM, Feijge MA, Reutelingsperger CP, Lindhout T. Collagen but not fibrinogen surfaces induce bleb formation, exposure of phosphatidylserine, and procoagulant activity of adherent platelets: evidence for regulation by protein tyrosine kinase-dependent $\mathrm{Ca}^{2+}$ responses. Blood. 1997;90:2615-2625.

33. Siljander PR, Munnix IC, Smethurst PA, Deckmyn H, Lindhout T, Ouwehand WH, Farndale RW, Heemskerk JW. Platelet receptor interplay regulates collagen-induced thrombus formation in flowing human blood. Blood. 2004;103:1333-1341.

34. Van Gorp RM, Feijge MA, Vuist WM, Rook MB, Heemskerk JW. Irregular spiking in free calcium concentration in single, human platelets. Regulation by modulation of the inositol trisphosphate receptors. Eur J Biochem. 2002;269:1543-1552.

35. Nergiz-Unal R, Lamers MM, Van Kruchten R, Luiken JJ, Cosemans JM, Glatz JF, Kuijpers MJ, Heemskerk JW. Signaling role of $\mathrm{CD}_{3} 6$ in platelet activation and thrombus formation on immobilized thrombospondin or oxidized low-density lipoprotein. J Thromb Haemost. 2011;9:1835-1846.

36. Dempfle CE, Argiriou S, Kucher K, Muller-Peltzer H, Rubsamen K, Heene DL. Analysis of fibrin formation and proteolysis during intravenous administration of ancrod. Blood. 2000;96:2793-2802.

37. Heemskerk JW, Bevers EM, Lindhout T. Platelet activation and blood coagulation. Thromb Haemost. 2002;88:186-193.

38. Heemskerk JW, Feijge MA, Henneman L, Rosing J, Hemker HC. The $\mathrm{Ca}^{2+}$-mobilizing potency of a-thrombin and thrombin-receptor-activating peptide on human platelets: concentration and time effects of thrombin-induced $\mathrm{Ca}^{2+}$ signaling. Eur J Biochem. 1997;249:547-555.

39. Razmara M, Hu H, Masquelier M, Li N. Glycoprotein Ilb/IIla blockade inhibits platelet aminophospholipid exposure by potentiating translocase and attenuating scramblase activity. Cell Mol Life Sci. 2007;64:999-1008.

40. Schoenwaelder SM, Yuan Y, Josefsson EC, White MJ, Yao Y, Mason KD, O'Reilly LA, Henley KJ, Ono A, Hsiao S, Willcox A, Roberts AW, Huang DC, Salem HH, Kile BT, Jackson SP. Two distinct pathways regulate platelet phosphatidylserine exposure and procoagulant function. Blood. 2009;114:663666.

41. Li Z, Delaney MK, O’Brien KA, Du X. Signaling during platelet adhesion and activation. Arterioscler Thromb Vasc Biol. 2010;30:2341-2349.

42. Dachary-Prigent J, Pasquet JM, Nurden AT. Simultaneous detection of changes in cytoplasmic $\mathrm{Ca}^{2+}$, aminophospholipid exposure and micro-vesiculation in activated platelets. Platelets. 1997;8:405412 .

43. Zhang J, Billingsley ML, Kincaid RL, Siraganian RP. Phosphorylation of Syk activation loop tyrosines is essential for Syk function. An in vivo study using a specific anti-Syk activation loop phosphotyrosine antibody. J Biol Chem. 2000;275:35442-35447.

44. Goncalves I, Hughan SC, Schoenwaelder SM, Yap CL, Yuan Y, Jackson SP. Integrin $a_{11 b} \beta_{3}$-dependent calcium signals regulate platelet-fibrinogen interactions under flow. Involvement of phospholipase 
Cү2. J Biol Chem. 2003;278:34812-34822.

45. Ablooglu AJ, Kang J, Petrich BG, Ginsberg MH, Shattil SJ. Antithrombotic effects of targeting $\alpha_{11 b} \beta_{3}$ signaling in platelets. Blood. 2009;113:3585-3592.

46. Falati S, Gross P, Merrill-Skoloff G, Furie BC, Furie B. Real-time in vivo imaging of platelets, tissue factor and fibrin during arterial thrombus formation in the mouse. Nat Med. 2002;8:1175-1181.

47. Hechler B, Nonne C, Eckly A, Magnenat S, Rinckel JY, Denis CV, Freund M, Cazenave JP, Lanza F, Gachet C. Arterial thrombosis: relevance of a model with two levels of severity assessed by histologic, ultrastructural and functional characterization. J Thromb Haemost. 2010;8:173-184.

48. Kuijpers MJ, Munnix IC, Cosemans JM, Vlijmen BV, Reutelingsperger CP, Egbrink MO, Heemskerk JW. Key role of platelet procoagulant activity in tissue factor-and collagen-dependent thrombus formation in arterioles and venules in vivo differential sensitivity to thrombin inhibition. Microcirculation. 2008;15:269-282.

49. Wonerow P, Pearce AC, Vaux DJ, Watson SP. A critical role for phospholipase $C \gamma 2$ in $\alpha_{11 b} \beta_{3}$-mediated platelet spreading. J Biol Chem. 2003;278:37520-37529.

50. Van der Meijden PE, Schoenwaelder SM, Feijge MA, Cosemans JM, Munnix IC, Wetzker R, Heller $\mathrm{R}$, Jackson SP, Heemskerk JW. Dual $\mathrm{P}_{2} \mathrm{Y}_{12}$ receptor signaling in thrombin-stimulated platelets: involvement of phosphoinositide 3-kinase $\beta$ but not gamma isoform in $\mathrm{Ca}^{2+}$ mobilization and procoagulant activity. FEBS J. 2008;275:371-385.

51. Boylan B, Gao C, Rathore V, Gill JC, Newman DK, Newman PJ. Identification of FcyRIla as the ITAM-bearing receptor mediating $\alpha_{11 b} \beta_{3}$ outside-in integrin signaling in human platelets. Blood. 2008;112:2780-2786.

52. Cosemans JM, Schols SE, Stefanini L, De Witt S, Feijge MA, Hamulyák K, Deckmyn H, Bergmeier W, Heemskerk JW. Key role of glycoprotein Ib/V/IX and von Willebrand factor in platelet activationdependent fibrin formation at low shear flow. Blood. 2011;117:651-660.

53. Suzuki-Inoue K, Wilde Jl, Andrews RK, Auger JM, Siraganian RP, Sekiya F, Rhee SG, Watson SP. Glycoproteins $\mathrm{VI}$ and Ib-IX-V stimulate tyrosine phosphorylation of tyrosine kinase Syk and phospholipase $C \gamma 2$ at distinct sites. Biochem J. 2004;378:1023-1029.

54. Hughes CE, Pollitt AY, Mori J, Eble JA, Tomlinson MG, Hartwig JH, O'Callaghan CA, Futterer K, Watson SP. CLEC-2 activates Syk through dimerization. Blood. 2010;115:2947-2955. 


\section{Supplemental data}
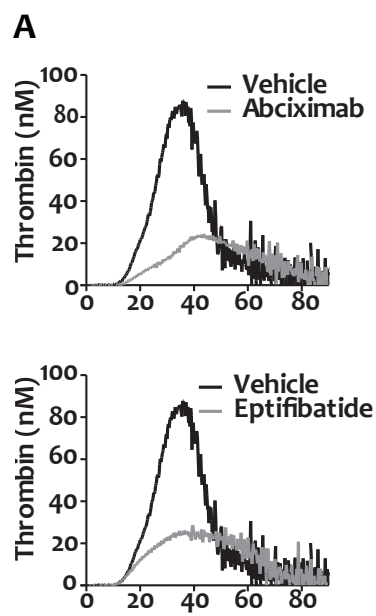
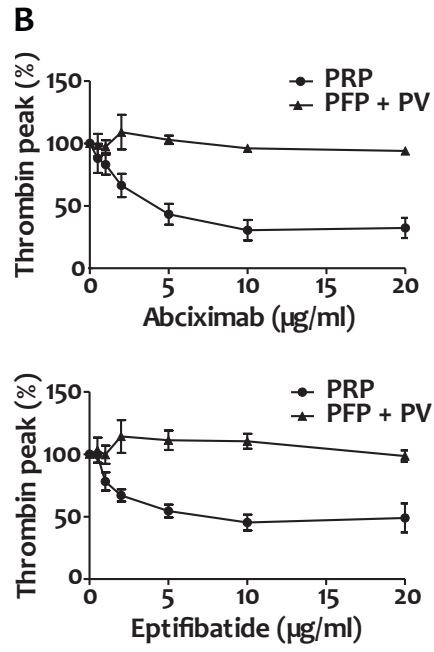

Supplemental Figure 1. Blocking of $\alpha_{11 b} \beta_{3}$ suppresses tissue factor-triggered thrombin generation in the presence of platelets. Human PRP $\left(1.0 \times 10^{8}\right.$ platelets $\left./ \mathrm{mL}\right)$ or PFP supplemented with phospholipid vesicles $(P V, 10 \mu \mathrm{M})$ was preincubated with vehicle, abciximab $(0.5-20 \mu \mathrm{g} / \mathrm{mL})$ or eptifibatide $(0.5-20$ $\mu \mathrm{g} / \mathrm{mL}$ ) for 20 minutes. Thrombin generation was stimulated with tissue factor (1 pM) and $\mathrm{CaCl}_{2}$. (A) Representative thrombin generation curves with PRP, showing comparable effects of all integrin blockers. (B) Dose-dependent inhibition of thrombin generation only in the presence of platelets. Means $\pm \operatorname{SEM}(n=3-9)$.
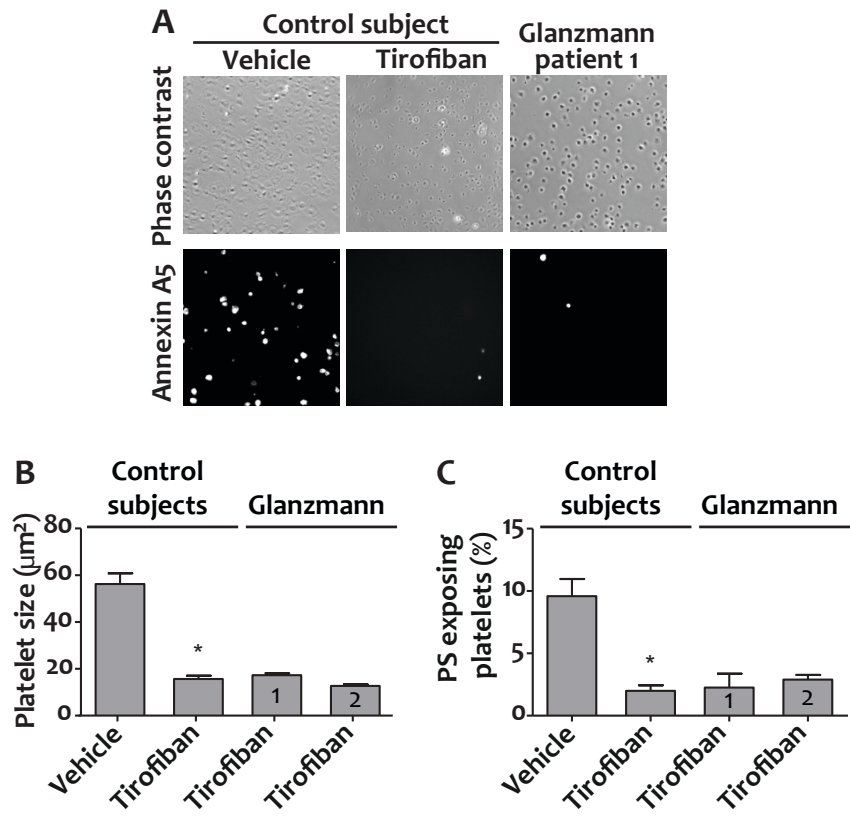

Supplemental Figure 2. Impaired spreading and thrombin activation of Glanzmann platelets. Platelets from healthy control subjects and Glanzmann patients were adhered to fibrinogen in the absence (control) or presence of tirofiban $(1 \mu \mathrm{g} / \mathrm{mL}$ ) for 30 minutes, and then stimulated with thrombin (10 $\mathrm{nM})$ for another 10 minutes. (A) Representative phase contrast $(120 \times 120 \mu \mathrm{m})$ and FITC-annexin A5 fluorescence $(150 \times 150 \mu \mathrm{m})$ images after thrombin activation. (B) Averaged platelet size; (C) fractions of PS-exposing platelets after activation. Means $\pm \operatorname{SEM}(n=3-6) ;{ }^{*} p<0.05$ versus vehicle. 
A

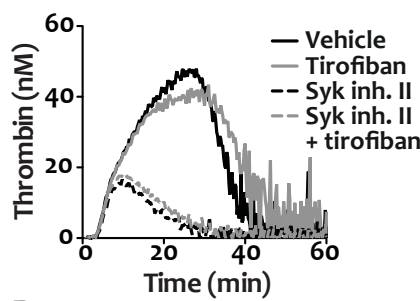

B

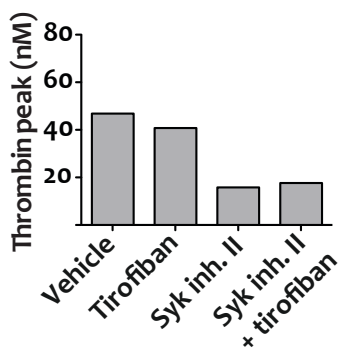

C

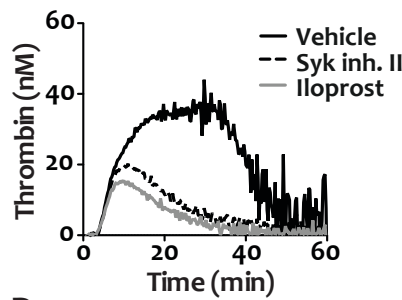

D

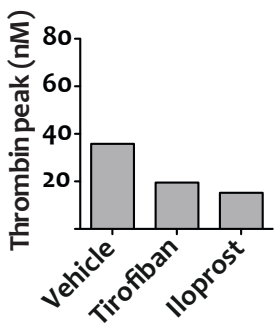

Supplemental Figure 3. Syk kinase is responsible for thrombin generation in tissue factor-stimulated PRP of a Glanzmann patient. PRP was incubated with vehicle, Syk inhibitor II (10 $\mu \mathrm{M})$, iloprost $(1 \mu \mathrm{M})$ and/ or tirofiban $(1 \mu \mathrm{g} / \mathrm{mL})$, and then stimulated with tissue factor $(1 \mathrm{pM})$ and $\mathrm{CaCl}_{2}$. Cangrelor (AR-C, $\left.10 \mu M\right)$ was present to eliminate $\mathrm{P}_{2} \mathrm{Y}_{12}$-dependent signalling events. (A, B) Effect of inhibitors on tissue factorinduced thrombin generation in PRP from blood samples collected on citrate, and (C, D) on citrate and tirofiban $(1 \mu \mathrm{g} / \mathrm{mL})$. Representative thrombin generation curves and mean thrombin peak heights of triplicate measurements are shown. 



\section{CHAPTER 6}

Platelet control of fibrin distribution and microelasticity in thrombus formation under flow

Swieringa F, Baaten $\mathrm{CC}^{*}$, Verdoold R*, Mastenbroek TG, Rijnveld N, Van der Laan KO, Breel EJ, Collins PW, Lancé MD, Henskens YM, Cosemans JM, Heemskerk JW, Van der Meijden PE (* equal contribution)

Arterioscler Thromb Vasc Biol. 2016; pii ATVBAHA.115.306537

Reprinted with permission 


\begin{abstract}
Platelet- and fibrin-dependent thrombus formation is regulated by blood flow and exposure of collagen and tissue factor. However, interactions between these bloodborne and vascular components are not well understood. Here, we developed a method to assess whole blood thrombus formation on microspots with defined amounts of collagen and tissue factor, allowing determination of the mechanical properties and intrathrombus composition. Confining the collagen content resulted in diminished platelet deposition and fibrin formation at high shear flow conditions, but this effect was compensated by a larger thrombus size and increased accumulation of fibrin in the luminal regions of the thrombi at the expense of the base regions. These thrombi were more dependent on tissue factor-triggered thrombin generation. Microforce nanoindentation analysis revealed a significantly increased microelasticity of thrombi with luminal-oriented fibrin. At a low shear rate, fibrin fibres tended to luminally cover the thrombi, again resulting in a higher microelasticity. Studies with blood from patients with distinct haemostatic insufficiencies indicated an impairment in formation of a platelet-fibrin thrombus in the cases of dilutional coagulopathy, thrombocytopenia, Scott syndrome and haemophilia B. Taken together, our data indicate that: (i) thrombin increases the platelet thrombus volume; (ii) tissue factor drives formation of fibrin outside of the platelet thrombus; (iii) limitation of platelet adhesion redirects fibrin from bottom to top of the thrombus; (iv) a lower shear rate promotes thrombus coverage with fibrin; $(v)$ the fibrin distribution pattern determines thrombus microelasticity; and (vi) the thrombus-forming process is reduced in patients with diverse haemostatic defects.
\end{abstract}

\title{
Introduction
}

During haemostasis, blood flow and platelet activation in combined action with the coagulation system determine the formation of platelet-fibrin thrombi at the injured vessel wall. Multiple components of the damaged endothelial and subendothelial layers are known to influence the dynamics of thrombus growth and fibrin polymerisation. ${ }^{1}$ Key vascular constituents triggering this process are collagen fibres and tissue factor (TF), serving as potent platelet- and coagulation-stimulating agents, respectively. ${ }^{2}$ Thrombin that is formed on the surface of activated platelets and on injured vascular cells is a central player in the haemostatic process by enhancing platelet activation, producing activated coagulation factors and converting fibrinogen into fibrin. ${ }^{3}$ Yet, the complex role of thrombin in formation of a platelet-fibrin thrombus under flow is at best incompletely studied.

Murine in vivo models of arterial thrombus formation have shown that the type and severity of vascular injury are determinative for the precise triggering of this process. Exposure of the vasculature to $\mathrm{FeCl}_{3}$, resulting in endothelial denudation, leads to collagen-dependent platelet activation with an additional role of TF, 
particularly upon milder $\mathrm{FeCl}_{3}$ injury. ${ }^{4-7}$ Thrombin and fibrin generation are elicited on procoagulant platelets exposing phosphatidylserine. ${ }^{8,} 9$ Platelets, furthermore, control the coagulation process by secreting pro- and anticoagulant proteins, whereas there is ample evidence for a role of platelet-exposed TF. ${ }^{10,11}$ On the other hand, in most laser-induced injury models, thrombus formation is primarily triggered by thrombin that is generated via vascular TF. ${ }^{12,13}$ Severe laser injury, however, also leads to collagen exposure and collagen-dependent platelet activation. ${ }^{14,15}$

Together, these in vivo findings indicate that the relative availability of plateletadhesive (like collagen) and coagulation-triggering (like TF) substances determines the buildup of a thrombus and, by implication, the roles of thrombin and fibrin herein. In support of this idea, different patterns of fibrin deposition have been detected in injury models, such as fibrin structures around a thrombus, ${ }^{16}$ and thrombin/fibrinrich areas in the thrombus core region. ${ }^{17,18}$ How the platelet- and coagulant-activities of the thrombogenic surface, for example, the relative abundance of collagen and TF, influence the buildup and composition of a platelet-fibrin thrombus has not been examined so far.

Viscoelasticy is considered to be an important haemostatic property of a fibrincontaining thrombus. For instance, in patients with severe factor XI deficiency, a low density fibrin network is associated with impaired haemostasis. ${ }^{19,}{ }^{20}$ However, the conditions that favour high elasticity of a platelet-fibrin thrombus or clot are largely unknown. The limited evidence available suggests that a local, high concentration of thrombin enforces the fibrin network, ${ }^{21}$ and that blood flow supports the alignment of stiff fibrin fibres. ${ }^{22,}{ }^{23}$ Macroscopic thromboelastic studies have not come much further than demonstrating that, in patients with dilutional coagulopathy, the overall clot strength is predictive for the risk of perioperative bleeding. ${ }^{24}$

In the present article, we investigated how the type of thrombogenic surface - with defined relative amounts of collagen and TF - affects the formation and composition of platelet-fibrin thrombi that are formed under high and low shear flow conditions. We developed standardised protocols, in which citrate-anticoagulated blood was flowed over defined collagen/TF microspots, and the deposition of platelets and fibrin(ogen) were assessed simultaneously. We then determined the distribution of fibrin within and outside the thrombus by confocal microscopy, as well as the microelasticity of the formed thrombi by a novel method of nanoindentation. The data reveal a surface- and flow-dependent thrombus buildup with a different location of fibrin.

\section{Materials and Methods}

Materials and Methods are available in the Supplemental data. 


\section{Results}

Collagen-TF surface determines buildup of platelet-fibrin thrombus and extent of fibrin formation under flow

To study the roles of platelets and coagulation in whole blood thrombus formation under flow, we applied a previously developed microspot method of thrombus formation in parallel-plate flow chambers, ${ }^{25}$ which was adapted to operate under strictly controlled coagulant conditions. The changes included: (i) preparation of microspots with defined amounts of collagen and tissue factor (TF), (ii) co-infusion of citrate-anticoagulated blood with $\mathrm{Mg}^{2+} / \mathrm{Ca}^{2+}$ recalcification medium to obtain physiological concentrations of divalent cations; (iii) adequate mixing of blood with medium by pushing through a tube-slit converter in the flow chamber, and (iv) realtime confocal recording of both platelet deposition and fibrin generated on the microspots using 3,3'-dihexyloxacarbocyanine ( DiOC $_{6}$ ) and Alexa Fluor (AF)647fibrinogen as probes, which were pre-added to the blood samples (Supplemental Fig. 1).

Pilot data indicated that co-coating of $10 \mathrm{pg}$ TF per microspot resulted in optimal shortening of time to fibrin formation, as checked with bright-field image recording, such in agreement with published findings. ${ }^{26}$ Perfusion of blood samples at a shear rate of $1,000 \mathrm{~s}^{-1}$ over microspots enriched in collagen (100 ng per microspot) resulted in a rapid formation of platelet thrombi that were rich in fibrin (Fig. $1 \mathrm{~A}-\mathrm{C}$ ). Lowering the collagen content ( $20 \mathrm{ng}$ per microspot) significantly decreased platelet deposition after 7 minutes of perfusion (area covered, $12.3 \pm 1.4 \%$ vs. $33.8 \pm 3.0 \%$; $p=0.005$ ) and delayed the onset of fibrin formation ( 3 vs. 5 minutes), as compared to the high collagen microspots (Fig. $1 \mathrm{~A}-\mathrm{C}$ ). In the absence of TF, when coagulation is triggered via the intrinsic pathway, ${ }^{27}$ platelet deposition was decreased on high collagen but unaltered on low collagen, whereas fibrin formation was greatly delayed in either case (Supplemental Table 1). Pretreatment of the blood with corn trypsin inhibitor to block the intrinsic coagulation pathway resulted in a delayed formation of fibrin only for microspots not containing TF (data not shown). In the absence of collagen (only $T F$ ), neither platelets nor fibrin deposited on the surface.

Reconstruction of stacks of confocal images in $3 \mathrm{D}$ indicated that the plateletfibrin thrombi formed on high collagen microspots were relatively small and dense, when compared with the more dispersed thrombi formed on low collagen microspots (Fig. 1A, D). Persistent thrombin activity on each type of microspot was confirmed by the addition of a fluorogenic thrombin substrate, which was continuously cleaved (data not shown). Markedly, with hirudin added to the blood, platelet deposition decreased on high collagen microspots (surface area coverage from 33.8 to $19.1 \%$, $p=0.001$ ), whereas on low collagen hardly any platelets were left (from 12.3 to 2.9 $\%, p<0.0001)$. As expected, with hirudin present, fibrin formation was completely suppressed on either surface (Fig. 1E). Quantification also showed that hirudin 
A

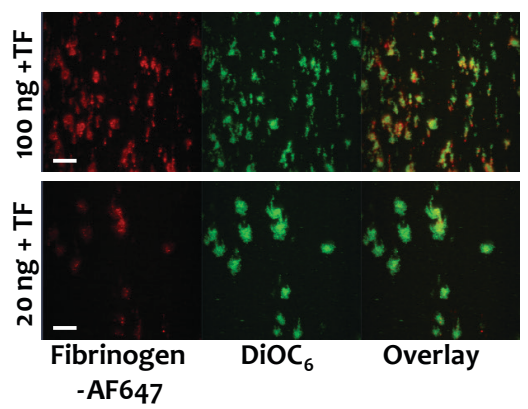

B
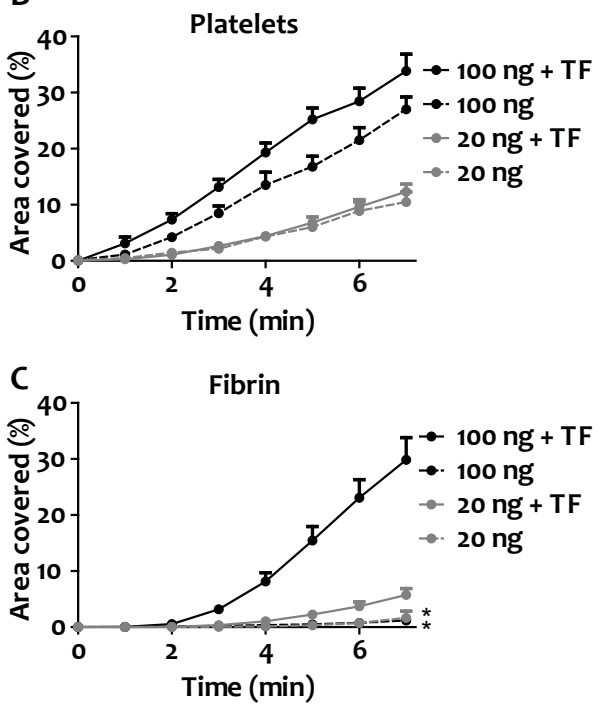

D

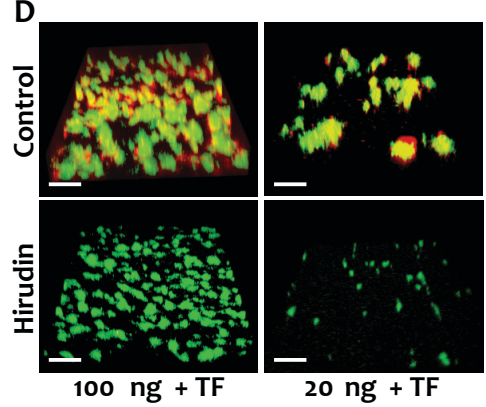

E
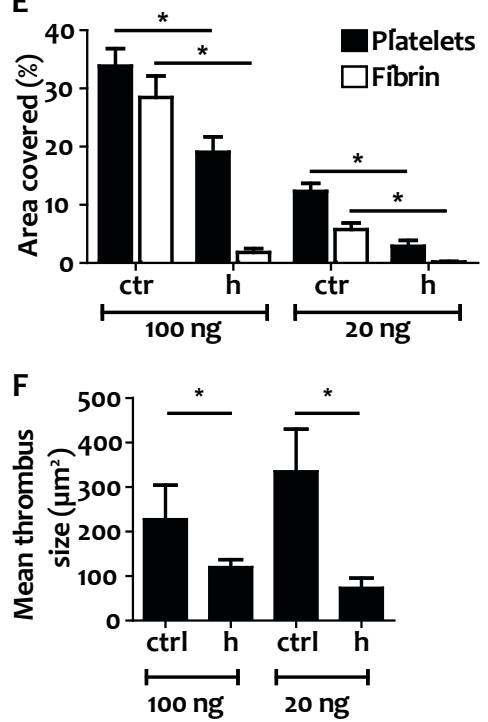

Figure 1. Collagen-tissue factor (TF) coating determines the buildup of platelet-fibrin thrombus formed under flow. Citrated whole blood was perfused during recalcification over microspots with collagen (20 or $100 \mathrm{ng}$ ) in the absence or presence of TF (10 pg) for 7 minutes at a wall shear rate of 1,000 $\mathrm{s}^{-1}$. Blood samples were prelabelled with $\mathrm{DiOC}_{6}$ to label platelets (green) and with AF647-fibrinogen to monitor fibrin formation (red). Control samples were pretreated with hirudin ( $h, 3 \mu \mathrm{g} / \mathrm{mL})$, where indicated. Twocolour microscopic confocal images were recorded in real time at frequency of $1 \mathrm{~Hz}$. (A) Representative fluorescence images from collagen/TF microspots after 7 minutes of flow (bars, $50 \mu \mathrm{m}$ ). (B, C) Timedependent accumulation of (B) DiOC 6 -labelled platelets and (C) AF647-fibrin(ogen) during blood flow. (D) Representative stacks of thrombi in 3D (z-step $1 \mu \mathrm{m}$ ) of platelets (green) and fibrin(ogen) (red) on collagen/TF microspots after 7 minutes of flow (bars, $50 \mu \mathrm{m}$ ). (E) Fluorescence area covered by platelets and fibrin on collagen/TF microspots after 7 minutes of flow. (F) Average thrombus size on spot as determined by morphometric image analysis. Means \pm SEM $(n=6-15),{ }^{*} p<0.05$.

significantly reduced the size of individual thrombi on the high collagen surface (from $228 \pm 76$ to $121 \pm 16 \mu \mathrm{m}^{2}, p=0.007$ ), a reduction that was even more pronounced on the low collagen surface (from $335 \pm 94$ to $74 \pm 21 \mu \mathrm{m}^{2}, p<0.001$ ) (Fig. 1F).

As microscopic images showed a gradual contraction of platelets in fibrincontaining thrombi, we aimed to quantify this process by addition of $3 \% \mathrm{DiOC}_{6}$-labelled 

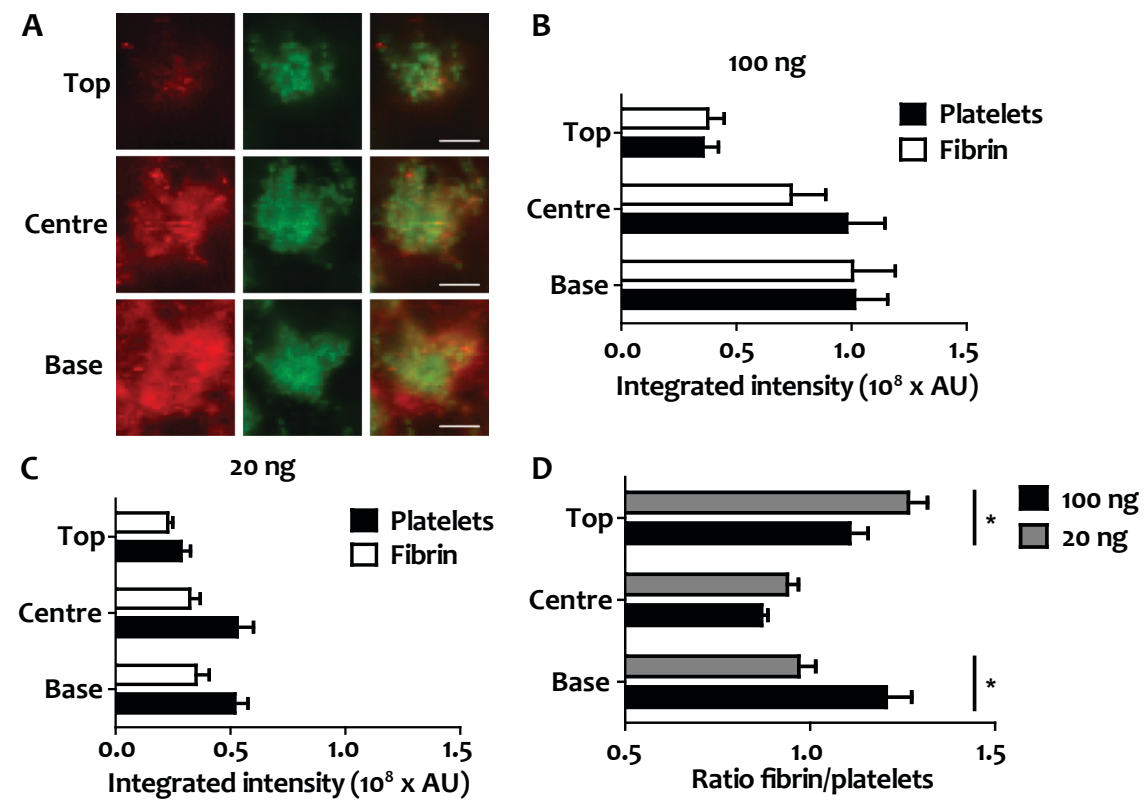

Figure 2. Collagen-tissue factor (TF) coating determines distribution of fibrin through a thrombus. Platelet-fibrin thrombi were formed by blood flow over microspots of collagen (20 or $100 \mathrm{ng}$ ) plus TF $(10 \mathrm{pg}$ ) for 7 minutes, as described in Figure 1. Confocal images of DiOC 6 (green) and AF647-fibrin(ogen) (red) fluorescence in 3D were captured. (A) Representative fluorescence images of optical slices at base, centre and top of thrombus on collagen (100 ng)/TF microspots (bars, $20 \mu \mathrm{m}$ ). (B, C) Integrated fluorescence intensity (arbitrary units, AU) of platelet (green) and fibrin (red) fluorescence from z stacks at base $(<10 \mu \mathrm{m})$, centre $(10-20 \mu \mathrm{m})$ and top $(>20 \mu \mathrm{m})$ of thrombi formed. (D) Ratio of platelet/fibrin fluorescence intensity at base, centre and top of thrombi. Means $\pm \operatorname{SEM}(n=6-7),{ }^{*} p<0.05$.

washed platelets to the blood, and then monitoring the movement of adhered platelets in time. It seemed that the overall displacement rate of platelets was high on microspots with collagen/TF during the time of fibrin formation $\left(1.06 \pm 0.11 \times 10^{-3} \mu \mathrm{m} / \mathrm{s}\right)$, whereas it was non-significantly changed $\left(0.87 \pm 0.10 \times 10^{-3} \mu \mathrm{m} / \mathrm{s}\right)$ in the presence of Gly-Pro-Arg-Pro, inhibiting fibrin polymerisation, and significantly lowered in the presence of hirudin $\left(0.64 \pm 0.05 \times 10^{-3} \mu \mathrm{m} / \mathrm{s}, p=0.0064\right)$. Hence, under the present flow conditions, platelet contraction did occur in the absence of thrombin or fibrin but was enhanced by the formation of either. This resolves a dispute in the literature that platelet-fibrin retraction is considered a thrombin-dependent event ${ }^{28}$ but can still occur under conditions where thrombin is blocked. ${ }^{29}$

By lowering the wall shear rate from 1,000 to $150 \mathrm{~s}^{-1}$, platelet deposition on high or low collagen was reduced (Supplemental Table 1), as expected due to a diminished role of von Willebrand factor. ${ }^{25}$ On the other hand, total fibrin formation did not reduce at lower shear rate (Supplemental Fig. 2). Co-localisation analysis of fluorescence from platelets and fibrin indicated major extension of the fibrin fibres outside of platelet aggregates especially at low shear conditions (150 $\left.\mathrm{s}^{-1}\right)$, and to a lesser extent at the higher shear rate (Supplemental Fig. 2). Jointly, these data indicate 
that limitation of the platelet-adhesive collagen surface resulted in an overall reduced platelet deposition, which was partly compensated by a larger thrombus size because of the activity of TF-triggered thrombin generation. Lowering of the shear rate also reduced platelet accumulation but in a relative way promoted fibrin formation.

\section{Collagen-TF surface and shear rate define distribution of fibrin in a thrombus}

To assess the amount and localisation of thrombus-associated fibrin on different microspots, $z$ stacks of confocal images of the fluorescent-labelled platelets and fibrin were captured at the end of high shear flow experiments (Fig. 2A). On microspots with high collagen, the majority of platelet and fibrin fluorescence was located near the base $(0-10 \mu \mathrm{m})$ and centre regions $(10-20 \mu \mathrm{m})$ of the thrombi. On microspots with low collagen, overall thrombus volume was reduced from $1.10 \pm 0.20$ to $0.46 \pm 0.10$ $\mathrm{x} 10^{6} \mu \mathrm{m}^{3}(p<0.001$; Supplemental Table 1$)$. In this case, fluorescence from platelets and fibrin was more evenly distributed among the base, centre and top regions of the thrombus (Fig. 2B, C). Markedly, the top regions of thrombi formed on low collagen showed a significantly higher ratio of fibrin to platelet fluorescence $(p=0.022)$, compared with those on high collagen (Fig. 2D). In contrast, the base regions of thrombi formed on low collagen had a lower fibrin/platelet ratio $(p=0.013)$, compared to the high collagen microspots.

The images were also analysed for co-localisation of platelets and fibrin, in order to assess the appearance of fibrin outside the aggregated platelets. On high collagen microspots, more fibrin fibres extended outside the platelet area at the base of the thrombi ( $28.8 \pm 1.9 \%$ of fibrin-positive pixels) than at the centre and top regions of the thrombus ( $8.9 \pm 2.1 \%$ and $5.3 \pm 1.8 \%$ fibrin-positive pixels, respectively; $p<0.001)$. Fibrin outgrowth was further restricted on low collagen microspots, where only 9.7 $\pm 2.8 \%, 2.3 \pm 0.6 \%$ and $5.2 \pm 1.1 \%$ of the fibrin fluorescence were detected outside the platelet aggregates, at the base, centre or top regions of thrombi, respectively.

Interestingly, when compared with a high shear rate, at a low shear rate of $150 \mathrm{~s}^{-1}$, overall thrombus volume reduced from $1.10 \pm 0.20$ to $0.78 \pm 0.02 \times 10^{6} \mu \mathrm{m}^{3}(p$ $=0.032)$ on high collagen and from $0.46 \pm 0.10$ to $0.29 \pm 0.04 \times 10^{6} \mu \mathrm{m}^{3}(p=0.026)$ on low collagen. At low shear rate, the majority of the fibrin was located outside of the platelet regions, which were low in height, such as apparent from analysis of $z$ stacks of confocal images (Supplemental Table 1).

\section{Collagen-TF surface and shear rate influence microelasticity of a thrombus}

A novel method of ferrule-top nanoindentation, related to atomic force microscopy, was used to determine how the quantity and localisation of fibrin fibres affected the mechanical and microelastic properties of the thrombi formed on microspots. This method is schematised in Fig. 3A, and described in more detail in the Supplement data. By gradually indenting and retracting a nanoindentation tip at multiple spots 

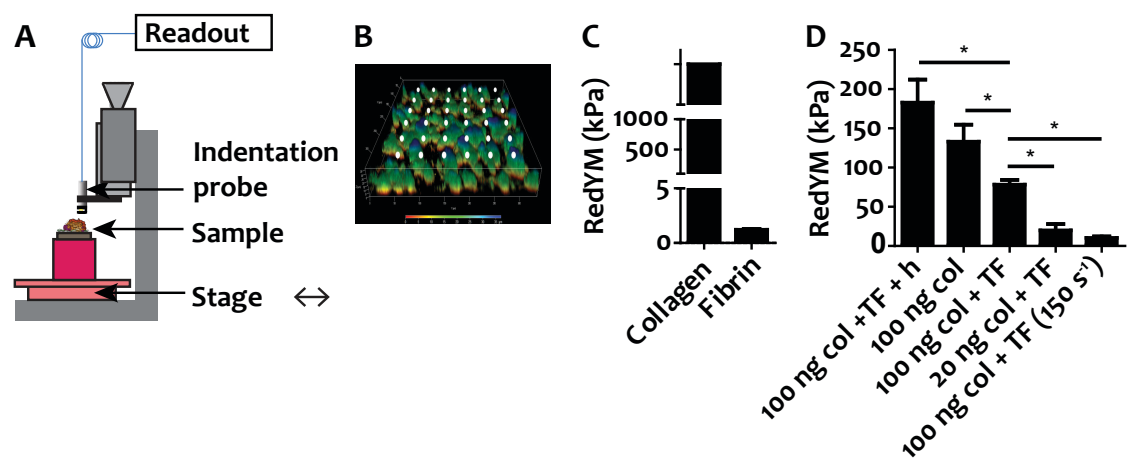

Figure 3. Collagen-tissue factor (TF) coating determines microelasticity of platelet-fibrin thrombus formed under flow. Platelet-fibrin thrombi were formed on microspots with collagen $\pm T F$, as described in Figure 1. Flow experiments were carried out at shear rate of $1,000 \mathrm{~s}^{-1}$, except where indicated otherwise $\left(150 \mathrm{~s}^{-1}\right)$. After careful rinse with Hepes buffer $\mathrm{pH}$ 7.5, coverslips with thrombi were subjected to nanoindentation ( $6 \times 6$ indentations/microspot). Control coverslips were used for scanning electron microscopy. (A) Schematic presentation of nanoindenter, featuring the indentation probe, sample and automated $x$ - $y$ stage. (B) Schematic grid pattern of preset $6 \times 6$ indentations per microspot with height gradient. (C) Reduced Young's modulus (RedYM, $\mathrm{kPa}$ ), a parameter inversely related to the microelasticity, in control measurements with a collagen-coated surface or with fibrinated plasma $(n=$ 16). (D) RedYM assessed for thrombi formed on indicated collagen/TF surfaces $(n=36-72)$. Means \pm SEM, ${ }^{*} p<0.05$, h indicates hirudin.

per coverslip, loading and unloading curves could be obtained, wherein slopes of the unloading curves informed on the reduced Young's modulus (RedYM), as an inverse measure for the microelasticity of the sample (Supplemental Fig. 3). By applying multiple nanoindentations per microspot surface in a grid-like pattern, high-resolution information on the microelasticity of the thrombi on the surface could be obtained (Fig. 3B). Uncoated (not shown) or collagen-coated coverslips without thrombi gave a high RedYM of $>1,000 \mathrm{kPa}$ (Fig. $3 \mathrm{C}$ ). In contrast, coverslips covered with coagulated, fibrin-containing plasma produced a low RedYM of $1.22 \pm 0.1 \mathrm{kPa}$, thus pointing to high microelasticity of the fibrin network. Nanoindentation measurements of thrombi formed on high collagen $(78.2 \pm 5.9 \mathrm{kPa})$ and low collagen $(20.0 \pm 8.1 \mathrm{kPa})$ microspots resulted in different RedYM values $(p=0.019)$, indicating that the latter had a higher microelasticity (Fig. 3D). Similarly, for thrombi formed on high collagen, lowering of the shear rate from $1,000 \mathrm{~s}^{-1}$ to $150 \mathrm{~s}^{-1}$ resulted in a lower RedYM $(p<0.0001)$ and hence a higher microelasticity (Fig. 3D). This suggested that intrathrombus fibrin increased the microelasticity of the sample. This was confirmed by nanoindentation analysis of fibrin-poor thrombi, formed on collagen without TF, giving high RedYM values of $133.1 \pm 21.6 \mathrm{kPa}$. In the presence of hirudin to block thrombin activity, and hence fibrin formation, the RedYM even further increased to $182.9 \pm 29.3 \mathrm{kPa}$ (Fig. $3 \mathrm{D}$ ). Together, this indicates that the thrombi with fibrin in the top or with outside coverage of fibrin, being formed on low density collagen microspots or at low shear rate, display a higher microelasticity, when compared with thrombi in which fibrin is accumulated at the base. 

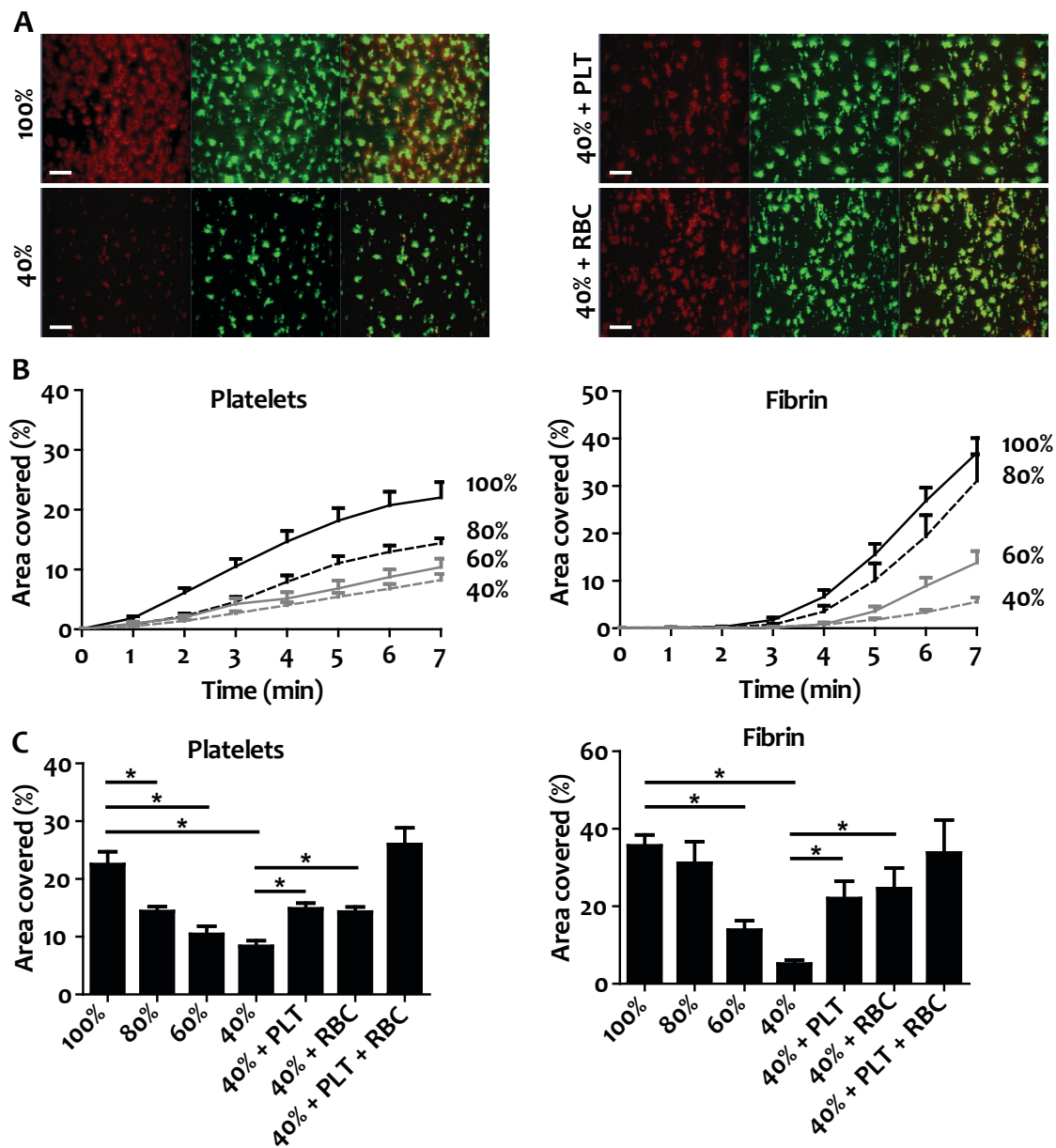

Figure 4. Restoration of platelet-fibrin thrombus formation by reconstitution of diluted blood. Platelet-fibrin thrombi were formed on microspots with collagen (100 ng) and tissue factor (TF; $10 \mathrm{pg}$ ), as described in Figure 1. Blood samples were diluted to indicated percentages with saline (keeping fixed concentrations of citrate, $\mathrm{MgCl}_{2}$ and $\left.\mathrm{CaCl}_{2}\right)$. Specific samples were reconstituted with autologous washed platelets (PLT) or red blood cells (RBC), added to the original counts. (A) Representative fluorescence images of $\mathrm{DiOC}_{6}$-labelled platelets (green) and AF647-fibrin(ogen) (red) after 7 minutes of flow using $100 \%$ or $40 \%$ blood with(out) reconstitution (bars, $50 \mu \mathrm{m}$ ). (B) Time-dependent accumulation of fluorescence from platelets (green) and fibrin (red) during perfusion. (C) Fluorescence area covered after 7 minutes of flow by platelets and fibrin. Means \pm SEM $(n=5-12),{ }^{*} p<0.05$.

\section{Formation of platelet-fibrin thrombus under conditions of impaired haemostasis}

Using several approaches, we further assessed the roles of platelets and other blood components in the formation of fibrin-containing thrombi. Considering the diminished clotting activity of patients with perioperative dilutional coagulopathy, ${ }^{30,31}$ we first determined the consequences of blood dilution in vitro. Blood samples were flowed at a shear rate of $1,000 \mathrm{~s}^{-1}$ over high collagen/TF microspots. In the absence of dilution, 
A

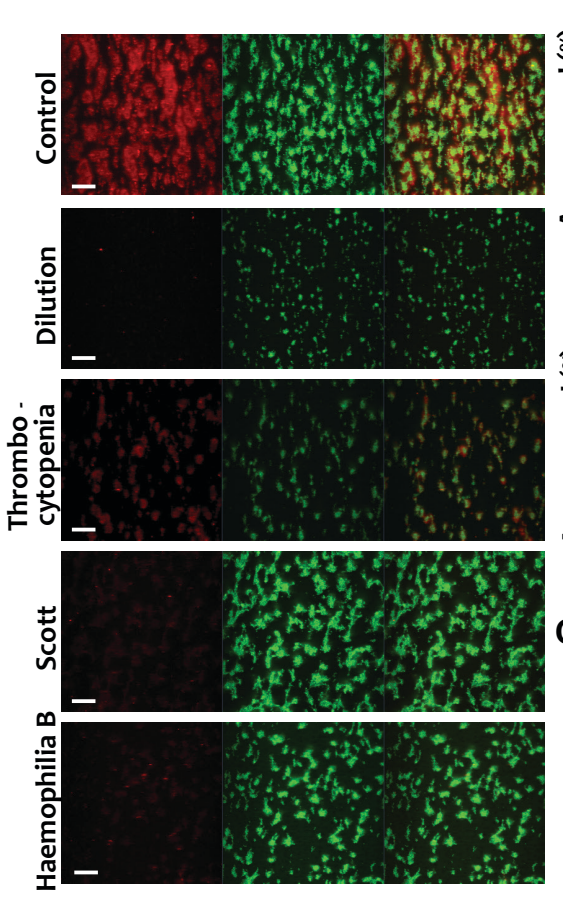

B
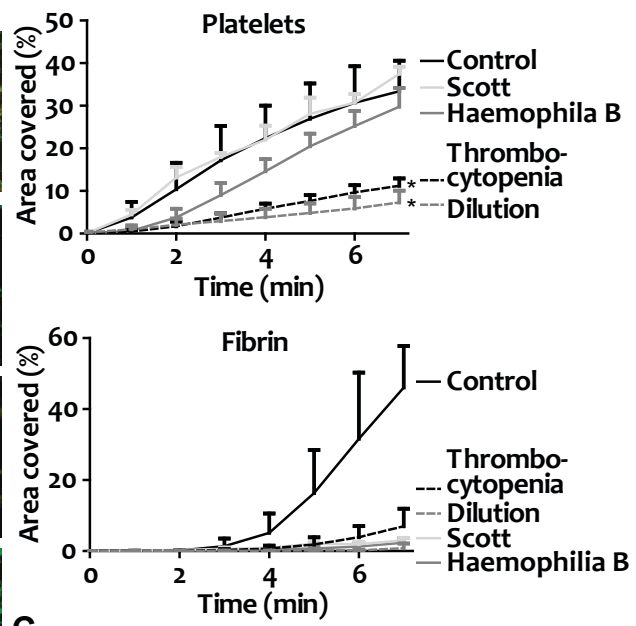

C

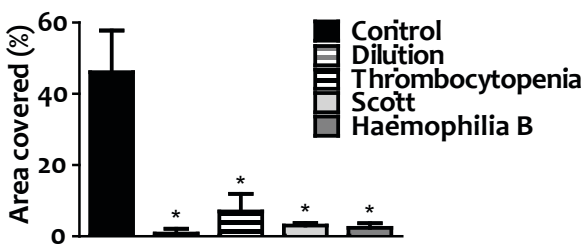

Figure 5. Impaired platelet-fibrin thrombus formation using blood from patients with impaired haemostasis. Blood was obtained from 7 healthy control subjects, 3 patients with dilutional coagulopathy because of massive fluid infusion during cardiothoracic surgery, 2 patients with acquired thrombocytopenia, 1 Scott syndrome, or 2 patients with haemophilia B. At least 3 blood samples were analysed per condition. Platelet-fibrin thrombi were formed in a standard way by blood flow at 1,000 $\mathrm{s}^{-1}$ over microspots with collagen (100 ng) and TF (10 pg), as described in Figure 1. (A) Representative images of $\mathrm{DiOC}_{6}$-labelled platelets (green) and AF647-fibrin(ogen) (red) after 7 minutes (bars, 50 $\mu \mathrm{m})$. (B) Time-dependent accumulation of platelet and fibrin fluorescence during blood perfusion. (C) Fluorescence area covered by fibrin after 7 minutes of flow. Means \pm SD $(n=6-9)$, *, significantly different from reference range of control blood samples.

this resulted in a high platelet deposition and fibrin formation (see above). However, dilution of the blood to 80,60 and $40 \%$ resulted in a gradual decrease in platelet deposition after 7 minutes of perfusion, amounting to $14 \pm 2 \%(p=0.046), 10 \pm 3 \%(p=$ $0.004)$ and $8 \pm 3 \%(p<0.001)$, respectively, in comparison with the undiluted sample. In contrast, amounts of fibrin only reduced at dilution to 60 and $40 \%$ blood, which reduction was accompanied by a prolonged time to onset of fibrin formation (Fig. 4A, B). Comparable results were obtained under conditions of a low, venous shear rate of $150 \mathrm{~s}^{-1}$ (Supplemental Fig. 4). Reconstitution of the $40 \%$ diluted blood with either washed platelets or red blood cells caused partial improvement of both platelet deposition and fibrin formation (Fig. 4A, C). Interestingly, complete recovery of the thrombus-forming process was obtained by addition of platelets in combination with red blood cells (Fig. 4C). These data pointed to a limiting role of platelet adhesion 
A
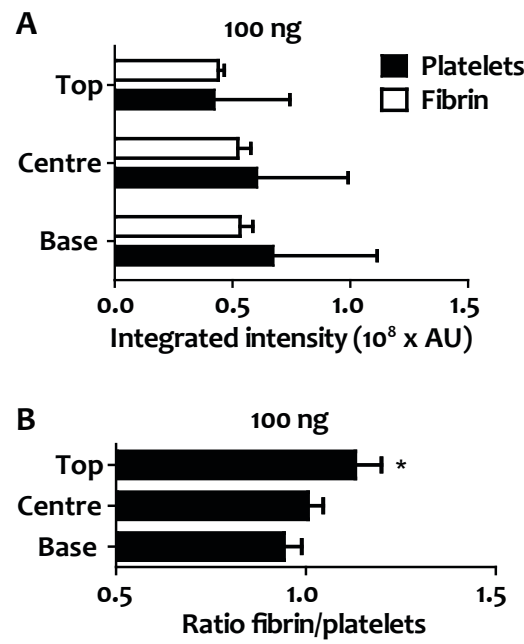

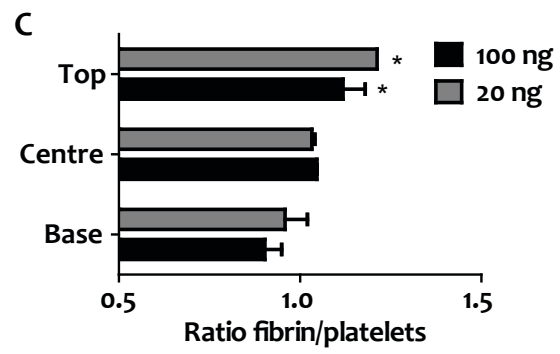

Figure 6. Altered fibrin distribution within thrombus formed by thrombocytopenic blood. Plateletfibrin thrombi were formed by blood flow over microspots with collagen (20 or $100 \mathrm{ng}$ ) and tissue factor (TF; $10 \mathrm{pg}$ ), as described in Figure 1. Confocal images of DiOC 6 (green) and AF647-fibrin(ogen) staining (red) in 3D were captured. (A) Integrated fluorescence intensity (arbitrary units, AU) of platelet (green) and fibrin (red) fluorescence from $z$ stacks at base $(>10 \mu \mathrm{m})$, centre $(10-20 \mu \mathrm{m})$ and top $(>20 \mu \mathrm{m})$ regions of thrombi after 7 minutes of blood perfusion on high collagen/TF. (B, C) Ratio of platelet/fibrin staining at base, centre and top of thrombi after 7 minutes on high collagen (B) or after 12 minutes (C) of blood perfusion on low and high collagen. Means \pm SD $(n=3),{ }^{*} p<0.05$ vs. base.

(enforced by marginalisation with erythrocytes) not only for platelet aggregation but also for fibrin formation under conditions of high shear flow. This was confirmed with scanning electron microscopy (Supplemental Fig. 5).

As a second approach, blood was used from various patients with haemostatic insufficiencies and a bleeding risk. Blood samples were obtained from 3 patients with dilutional coagulopathy because of massive fluid infusion during surgery. In this case, only few platelet aggregates of small size were formed and no fibrin was generated during the measured time of 7 minutes (Fig. 5A). With blood samples from 2 thrombocytopenic patients, platelet deposition was comparably low, although measurable amounts of fibrin were formed (Fig. 5A, C), such in accordance with normal coagulation activity in this condition. A requirement for platelet procoagulant activity (phosphatidylserine exposure) was investigated with blood samples from a Scott patient, whose platelets lack this property. ${ }^{32}$ Whereas platelet deposition and aggregate size were not affected, fibrin formation was essentially absent in the Scott blood samples. Similarly, impaired coagulation, as in blood from 2 patients with haemophilia B ( $\sim 5 \%$ factor IX), led to normal platelet aggregation under flow, but an impaired fibrin formation (Fig. 5A, C).

Because the flow of thrombocytopenic blood over high collagen microspots still gave limited fibrin formation, the intrathrombus localisation of fibrin could be analysed in more detail. Analysis of $z$ stacks of confocal images indicated that fibrin 
and platelets were similarly distributed throughout the thrombi (Fig. 6A). Yet, the ratio of fibrin to platelets was higher in the top regions of thrombi (Fig. 6B), with only limited fibrin extending from the platelet aggregates at base areas ( $12 \pm 8 \%$ of fibrinpositive pixels). This fibrin distribution pattern, concentrated in platelet regions, persisted even after prolongation of the perfusion time to 12 minutes (Fig. 6C). After 12 minutes, at high and low collagen microspots, fibrin deposition outside the platelet regions at the thrombus base gradually increased to $34 \pm 14 \%$ and $6 \pm 1 \%$, respectively. Jointly, these results pointed to platelet control of fibrin formation and distribution in thrombi formed on collagen/TF surfaces, relying on phosphatidylserine exposure.

\section{Discussion}

This study shows that, under conditions of high shear blood flow and coagulation, platelet deposition and platelet-dependent fibrin formation are decreased when collagen as a platelet-activating substrate becomes limited. Although fewer thrombi are formed on microspots with low collagen, we see a partly compensating effect in that thrombus size is increased and the contribution of thrombin to thrombus growth is relatively more important. Nanoindentation measuring viscoelastic properties of the sample indicates that the thrombi formed on low collagen possess enhanced microelasticity because of fibrin accumulation in the luminal region of thrombi. Interestingly, redistribution of fibrin from the base to the luminal region of thrombi is also observed under conditions of thrombocytopenia. Hence, a limitation of either the platelet-activating surface or the platelet number leads to reductions in platelet deposition and fibrin accumulation, which effects are accompanied by an altered fibrin distribution throughout the thrombus. Blood flow at low shear rate similarly enhances microelasticity by reducing platelet deposition and increasing the formation of a fibrin coat surrounding the thrombi.

Model of thrombus buildup, determining fibrin distribution and microelasticity

Platelet adhesion to collagen is known to result in a sustained rise in cytosolic calcium and phosphatidylserine exposure. This procoagulant surface greatly promotes the assembly of coagulation factors culminating in the formation of factor Xa and thrombin. ${ }^{2}$ Earlier, in flowing mouse blood, we have established that in the presence of collagen and TF this platelet-dependent factor Xa formation is essential for the propagation of fibrin formation and for boosting platelet phosphatidylserine exposure. ${ }^{3}$ The present results allow to extend this coagulation-platelet activation cross talk to the human system, in that we find that human deficiency in factor IX (haemophilia B patient) or deficiency in platelet phosphatidylserine exposure (Scott syndrome patient) results in almost complete abolition of fibrin formation under flow conditions.

Although in the past years progress has been made in determining the mechanical 


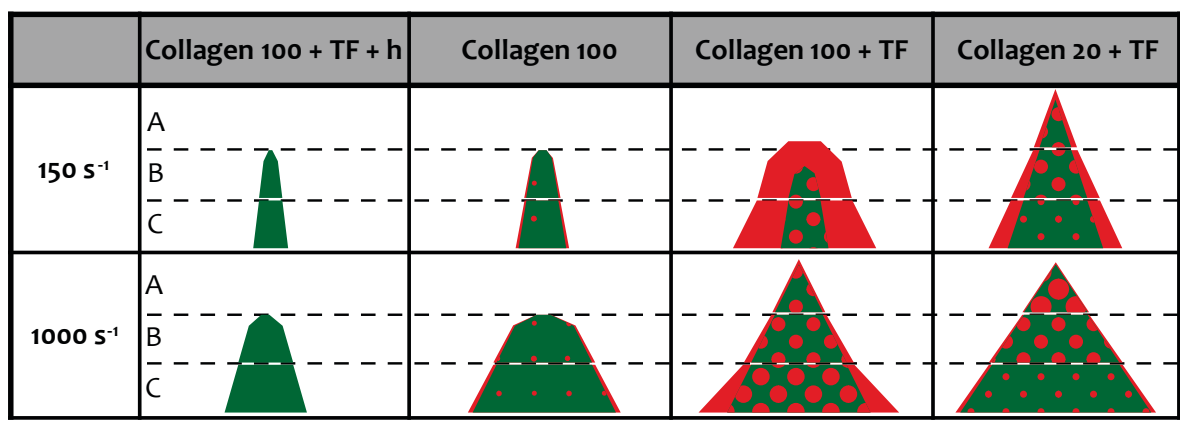

Figure 7. Schematic representation of thrombus buildup on collagen/TF surfaces. Platelet-fibrin thrombi were formed by blood perfusion over microspots with indicated amounts of collagen and TF at indicated shear rates, as described in Figure 1. In specific blood samples, thrombin was inhibited with hirudin $(+h)$. Multiple parameters of thrombus formation were assessed; for full data see Supplemental Table 1. Cartoons illustrate key characteristics of thrombi in terms of: width (scale 1-5), height (scale 1-3), fibrin inside platelet thrombus (sphere size: 1-4), and fibrin outside platelet thrombus (size of shell). Presentation is for three thrombus zones: A, $<10 \mu \mathrm{m}$ from microspot surface; $\mathbf{B}, 10-20 \mu \mathrm{m} ; \mathbf{C},>20 \mu \mathrm{m}$.

properties of fibrin fibres formed under static conditions, little is known about the elasticity of platelet-fibrin thrombi formed under flow. Recently, a new method of nanoindentation has been used to assess the microelasticity of clotted murine platelet-rich plasma. ${ }^{33}$ In this study, we have used this technique to measure the physical characteristics of human thrombi formed at high and low shear rates and find that thrombi with a high fibrin content in the thrombus top region are highest in microelasticity. Jointly, our results indicate that not so much the fibrin content, but rather the fibrin distribution through a thrombus determines its elastic properties. We hypothesise that the high microelasticity of thrombi with luminal-oriented fibrin ameliorates the haemostatic process. In agreement with this, thromboelastometry studies have indicated that a high clot elasticity associates with less bleeding in patients with haemostatic insufficiencies. ${ }^{20,34}$

If platelet adhesion is limited (low collagen), we see a relatively high fibrin content in the top region of an apparently 'loose' thrombus. On the other hand, at high platelet adhesion (high collagen), fibrin appears to be primarily formed at the base region of thrombi. Summarising these data as in Figure 7, we conclude that: (i) the presence of thrombin increases platelet-thrombus volume independent of the shear rate; (ii) the presence of TF drives fibrin formation outside of the thrombus; (iii) limitation of platelet adhesion redirects fibrin from the bottom to the top of the thrombus; (iv) lowering of the shear rate results in a more fibrin rich thrombus; and (v) the thrombus microelasticity is determined by the distribution pattern of fibrin.

\section{Comparison to in vivo models}

These findings provide an extension of the haemostatic thrombus model, based on in vivo observations, presented by the groups of Brass and Diamond, in which 
thrombin activity is confined to the dense thrombus core. ${ }^{18,35,36}$ Previous in vivo studies have shown that, upon laser-induced injury of arterioles, where exposed TF drives the thrombotic process, fibrin concentrates near the vessel wall and in the vascular-oriented part of the thrombus., ${ }^{6,14}$ Our findings suggest that this vascularoriented fibrin distribution pattern points to a relatively high abundance of plateletadhesive substrates. These substrates can be collagens, as well as other vascular components, such as laminins and von Willebrand factor. In other words, it seems that the 'strength' of the platelet-adhesive surface controls the platelet packing density during thrombus buildup, and thereby the thrombin retention and fibrin distribution pattern. Confirmative evidence for this hypothesis comes from in vivo studies using PAR4-deficient mice, in which fibrin was found to redistribute through the whole thrombus as a consequence of diminished platelet activation. ${ }^{16}$

A different pattern of fibrin distribution occurs under low shear flow conditions, where relatively more (microelastic) fibrin is formed, appearing as a coat that covers the thrombi. This might be explained by a reduction in the flow-dependent removal of thrombin and fibrin monomers. ${ }^{37}$ In accordance with our results, it has been shown that the thrombi formed in mesenteric venules after laser injury are surrounded by a fibrin cap. ${ }^{38}$

\section{Effects of haemostatic insufficiencies}

Under conditions of (perioperative) dilution, we find that platelet deposition is more sensitive to blood dilution than fibrin formation, which is impaired already at $60 \%$ blood. This contrasts to static measurements of macroscopic clot strength by thromboelastometry, which seemed to be less sensitive to dilution effects. ${ }^{30}$ Single-pass flow perfusion likely makes the difference here because under flow the deposited platelets provide a rate-limiting surface for the formation of thrombin and subsequent fibrin, whereas the same flow removes procoagulant factors and hence restricts fibrin formation. ${ }^{37}$ Remarkably, in reconstitution experiments, the addition of both platelets and red blood cells was required for complete reversal of dilution effects. By implication, this indicates that under conditions of flow and sufficient margination of platelets by red blood cells, a coagulant activity of $>60 \%$ is required for unrestricted generation of fibrin. These findings are supported by hydrodynamic simulations indicating that platelet interactions with the vessel wall are promoted by an increasing hematocrit. ${ }^{39}$

A limitation of the present study is the absence of vascular cells such as endothelial cells, which can also provide a procoagulant surface for the formation of thrombin..$^{40}$ On the other hand, current overviews indicate that collagen (TF)-based in vitro flow studies compare well with in vivo arterial thrombosis models, when evaluating the functional effects of genetic knockout in mice. ${ }^{41,42}$ Hence, we consider the present test of formation of platelet-fibrin thrombi under flow as a valid method to determine the haemostatic capacity of a blood sample. For fibrin localisation we used 
fluorescently labelled fibrinogen and therefor the distinction between fibrinogen and fibrin cannot be made unambiguously. We minimised the contribution of fibrinogen to the fibrin signal by threshold settings and the presence of fluorescent fibrin fibres was always confirmed from bright-field images. The advantage is that this method prevents underestimation of the fibrin signal as a consequence of poor permeation in the thrombus when using a fibrin antibody.

In summary, we conclude that under conditions of flow and coagulation the amount and localisation of fibrin are regulated by the relative abundance of triggers for platelet adhesion/activation (e.g. collagen) and coagulation (TF), and that the fibrin distribution is determinative for the clot microelasticity. Thrombin herein influences thrombus growth as well as fibrin formation, depending on its local concentration.

\section{Sources of funding}

Support for this work was obtained from the Dutch Landsteiner Foundation for Blood Transfusion Research (1006) and ZonMW, Animal-free Research Techniques (114021004). PvdM is supported by the Cardiovascular Center Maastricht.

\section{Disclosures}

NR, EJB and KL are employees of Optics11, Amsterdam, The Netherlands. Other authors have nothing to disclose.

\section{References}

1. Wolberg AS, Aleman MM, Leiderman K, Machlus KR. Procoagulant activity in hemostasis and thrombosis: Virchow's triad revisited. Anesth Analg. 2012;114:275-285.

2. Versteeg HH, Heemskerk JW, Levi M, Reitsma PH. New fundamentals in hemostasis. Physiol Rev. 2013;93:327-358.

3. Swieringa F, Kuijpers MJ, Lamers MM, Van der Meijden PE, Heemskerk JW. Rate-limiting roles of the tenase complex of factors VIII and IX in platelet procoagulant activity and formation of platelet-fibrin thrombi under flow. Haematologica. 2015;100:748-756.

4. Nieswandt B, Brakebusch C, Bergmeier W, Schulte V, Bouvard D, Mokhtari-Nejad R, Lindhout T, Heemskerk JW, Zirngibl H, Fassler R. Glycoprotein VI but not $\alpha_{2} \beta_{1}$ integrin is essential for platelet interaction with collagen. Embo J. 2001;20:2120-2130.

5. Massberg S, Gawaz M, Gruner S, Schulte V, Konrad I, Zohlnhofer D, Heinzmann U, Nieswandt B. A crucial role of glycoprotein VI for platelet recruitment to the injured arterial wall in vivo. $J$ Exp Med. 2003;197:41-49.

6. Dubois C, Panicot-Dubois L, Merrill-Skoloff G, Furie B, Furie BC. Glycoprotein VI-dependent and -independent pathways of thrombus formation in vivo. Blood. 2006;107:3902-3906.

7. Kuijpers MJ, Munnix IC, Cosemans JM, Vlijmen BV, Reutelingsperger CP, Egbrink MO, Heemskerk JW. Key role of platelet procoagulant activity in tissue factor-and collagen-dependent thrombus formation in arterioles and venules in vivo differential sensitivity to thrombin inhibition. Microcirculation. 2008;15:269-282.

8. Munnix IC, Kuijpers MJ, Auger J, Thomassen CM, Panizzi P, Van Zandvoort MA, Rosing J, Bock PE, 
Watson SP, Heemskerk JW. Segregation of platelet aggregatory and procoagulant microdomains in thrombus formation: regulation by transient integrin activation. Arterioscler Thromb Vasc Biol. 2007;27:2484-2490.

9. De Witt SM, Verdoold R, Cosemans JM, Heemskerk JW. Insights into platelet-based control of coagulation. Thromb Res. 2014;133 Suppl 2:S139-148.

10. Panes O, Matus V, Saez CG, Quiroga T, Pereira J, Mezzano D. Human platelets synthesize and express functional tissue factor. Blood. 2007;109:5242-5250.

11. Schwertz H, Tolley ND, Foulks JM, Denis MM, Risenmay BW, Buerke M, Tilley RE, Rondina MT, Harris EM, Kraiss LW, Mackman N, Zimmerman GA, Weyrich AS. Signal-dependent splicing of tissue factor pre-mRNA modulates the thrombogenicity of human platelets. J Exp Med. 2006;203:24332440.

12. Falati S, Gross P, Merrill-Skoloff G, Furie BC, Furie B. Real-time in vivo imaging of platelets, tissue factor and fibrin during arterial thrombus formation in the mouse. Nat Med. 2002;8:1175-1181.

13. Furie B, Furie BC. Mechanisms of thrombus formation. N Engl J Med. 2008;359:938-949.

14. Hechler B, Nonne C, Eckly A, Magnenat S, Rinckel JY, Denis CV, Freund M, Cazenave JP, Lanza F, Gachet C. Arterial thrombosis: relevance of a model with two levels of severity assessed by histologic, ultrastructural and functional characterization. J Thromb Haemost. 2010;8:173-184.

15. Bellido-Martin L, Chen V, Jasuja R, Furie B, Furie BC. Imaging fibrin formation and platelet and endothelial cell activation in vivo. Thromb Haemost. 2011;105:776-782.

16. Vandendries ER, Hamilton JR, Coughlin SR, Furie B, Furie BC. Par4 is required for platelet thrombus propagation but not fibrin generation in a mouse model of thrombosis. Proc Natl Acad Sci U S A. 2007;104:288-292.

17. Stalker TJ, Traxler EA, Wu J, Wannemacher KM, Cermignano SL, Voronov R, Diamond SL, Brass LF. Hierarchical organization in the hemostatic response and its relationship to the platelet-signaling network. Blood. 2013;121:1875-1885.

18. Stalker TJ, Welsh JD, Tomaiuolo M, Wu J, Colace TV, Diamond SL, Brass LF. A systems approach to hemostasis: 3. Thrombus consolidation regulates intrathrombus solute transport and local thrombin activity. Blood. 2014;124:1824-1831.

19. Wolberg AS. Thrombin generation and fibrin clot structure. Blood Rev. 2007;21:131-142.

20. Zucker M, Seligsohn U, Salomon O, Wolberg AS. Abnormal plasma clot structure and stability distinguish bleeding risk in patients with severe factor XI deficiency. J Thromb Haemost. 2014;12:11211130.

21. Neeves KB, Illing DA, Diamond SL. Thrombin flux and wall shear rate regulate fibrin fiber deposition state during polymerization under flow. Biophys J. 2010;98:1344-1352.

22. Collet JP, Shuman H, Ledger RE, Lee S, Weisel JW. The elasticity of an individual fibrin fiber in a clot. Proc Natl Acad Sci U S A. 2005;102:9133-9137.

23. Gersh KC, Edmondson KE, Weisel JW. Flow rate and fibrin fiber alignment. J Thromb Haemost. 2010;8:2826-2828.

24. Schols SE, Lancé MD, Feijge MA, Damoiseaux J, Marcus MA, Hamulyák K, Ten Cate H, Heemskerk JW, Van Pampus EC. Impaired thrombin generation and fibrin clot formation in patients with dilutional coagulopathy during major surgery. Thromb Haemost. 2010;103:318-328.

25. De Witt SM, Swieringa F, Cavill R, Lamers MM, Van Kruchten R, Mastenbroek T, Baaten C, Coort S, Pugh N, Schulz A, Scharrer I, Jurk K, Zieger B, Clemetson KJ, Farndale RW, Heemskerk JW, Cosemans JM. Identification of platelet function defects by multi-parameter assessment of thrombus formation. Nat Commun. 2014;5:4257.

26. Okorie UM, Denney WS, Chatterjee MS, Neeves KB, Diamond SL. Determination of surface tissue factor thresholds that trigger coagulation at venous and arterial shear rates: amplification of 100 fM circulating tissue factor requires flow. Blood. 2008;111:3507-3513.

27. Van der Meijden PE, Munnix IC, Auger JM, Govers-Riemslag JW, Cosemans JM, Kuijpers MJ, Spronk 
HM, Watson SP, Renné T, Heemskerk JW. Dual role of collagen in factor XII-dependent thrombus formation. Blood. 2009;114:881-890.

28. Shattil SJ, Kim C, Ginsberg MH. The final steps of integrin activation: the end game. Nat Rev Mol Cell Biol. 2010;11:288-300.

29. Ono A, Westein E, Hsiao S, Nesbitt WS, Hamilton JR, Schoenwaelder SM, Jackson SP. Identification of a fibrin-independent platelet contractile mechanism regulating primary hemostasis and thrombus growth. Blood. 2008;112:90-99.

30. Ninivaggi M, Feijge MA, Baaten CC, Kuiper GJ, Marcus MA, Ten Cate H, Lancé MD, Heemskerk JW, Van der Meijden PE. Additive roles of platelets and fibrinogen in whole-blood fibrin clot formation upon dilution as assessed by thromboelastometry. Thromb Haemost. 2014;111:447-457.

31. Schols SE, Feijge MA, Lancé MD, Hamulyák K, Ten Cate H, Heemskerk JW, Van Pampus EC. Effects of plasma dilution on tissue-factor-induced thrombin generation and thromboelastography: partly compensating role of platelets. Transfusion. 2008;48:2384-2394.

32. Castoldi E, Collins PW, Williamson PL, Bevers EM. Compound heterozygosity for 2 novel TMEM16F mutations in a patient with Scott syndrome. Blood. 2011;117:4399-4400.

33. Slaboch CL, Alber MS, Rosen ED, Ovaert TC. Mechano-rheological properties of the murine thrombus determined via nanoindentation and finite element modeling. J Mech Behav Biomed Mater. 2012;10:75-86.

34. Wolberg AS, Allen GA, Monroe DM, Hedner U, Roberts HR, Hoffman M. High dose factor VIIa improves clot structure and stability in a model of haemophilia B. Br J Haematol. 2005;131:645-655.

35. Tomaiuolo M, Stalker TJ, Welsh JD, Diamond SL, Sinno T, Brass LF. A systems approach to hemostasis: 2. Computational analysis of molecular transport in the thrombus microenvironment. Blood. 2014;124:1816-1823.

36. Welsh JD, Stalker TJ, Voronov R, Muthard RW, Tomaiuolo M, Diamond SL, Brass LF. A systems approach to hemostasis: 1 . The interdependence of thrombus architecture and agonist movements in the gaps between platelets. Blood. 2014;124:1808-1815.

37. Shen F, Kastrup CJ, Liu Y, Ismagilov RF. Threshold response of initiation of blood coagulation by tissue factor in patterned microfluidic capillaries is controlled by shear rate. Arterioscler Thromb Vasc Biol. 2008;28:2035-2041.

38. Kamocka MM, Mu J, Liu X, Chen N, Zollman A, Sturonas-Brown B, Dunn K, Xu Z, Chen DZ, Alber MS, Rosen ED. Two-photon intravital imaging of thrombus development. J Biomed Opt. 2010;15:016020.

39. Watts T, Barigou M, Nash GB. Comparative rheology of the adhesion of platelets and leukocytes from flowing blood: why are platelets so small? Am J Physiol Heart Circ Physiol. 2013;304:H14831494 .

40. Ivanciu L, Krishnaswamy S, Camire RM. New insights into the spatiotemporal localization of prothrombinase in vivo. Blood. 2014;124:1705-1714.

41. Mastenbroek TG, Van Geffen JP, Heemskerk JW, Cosemans JM. Acute and persistent platelet and coagulant activities in atherothrombosis. J Thromb Haemost. 2015;13 Suppl 1:S272-280.

42. Stegner D, Nieswandt B. Platelet receptor signaling in thrombus formation. J Mol Med (Berl). 2011;89:109-121.

\section{Significance}

Activation of blood platelets as well as the coagulation system is required for a normal haemostatic response. While it is known that platelet- and fibrin-dependent thrombus formation is regulated by blood flow, the blood composition and vascular triggers such as collagen and tissue factor, the interactions between these bloodborne and vascular components are not well understood. Our data imply that the 
relative abundance of vascular triggers of platelet adhesion/activation (collagen) at one side and of coagulation (tissue factor) at the other side is determining for both thrombus growth and thrombus composition, in particular regarding the distribution of fibrin. Furthermore, we show that the fibrin distribution is determinative for the thrombus microelasticity as assessed by nanoindentation. 


\section{Supplemental data}

\section{Methods}

Healthy volunteers and patients

Blood was taken from healthy volunteers and patients after informed consent was obtained in accordance with the Declaration of Helsinki. Studies were approved by the local medical ethics committee (METC 10-3-023). All donors were free from antiplatelet and anticoagulant medication for at least 2 weeks. Blood was taken from healthy control donors and from two patients with hemophilia B (both $5 \%$ factor IX), a patient with Scott syndrome (deficient anoctamin 6 expression), ${ }^{1}$ two patients with immune-induced thrombocytopenia (platelet count $85 \times 10^{9} / \mathrm{L}$ and $22 \times 10^{9} / \mathrm{L}$ ), or from three patients with dilutional coagulopathy due to massive fluid infusion during cardiothoracic surgery (4.5-5 L), collected in the operating theatre. At least three blood samples were analysed for all conditions.

\section{Blood collection and preparation}

Blood was collected in 1:10 (v/v) $3.2 \%$ trisodium citrate for whole blood perfusion experiments and the preparation of washed red blood cells, or in 1:6 (v/v) acidic citrate dextrose (ACD, $80 \mathrm{mM}$ trisodium citrate, $52 \mathrm{mM}$ citric acid and $180 \mathrm{mM}$ glucose) for the isolation of washed platelets. Platelets and red blood cells were isolated as described. ${ }^{2}$ Cell count was determined using a thrombocounter (Coulter Electronics, High Wycombe, United Kingdom).

\section{Formation of platelet-fibrin thrombi under conditions of coagulation and flow}

Thrombus formation under flow conditions was determined by perfusion of citrated whole blood over a collagen/tissue factor (TF) coated glass coverslip in a transparent parallel-plate perfusion chamber (width $3 \mathrm{~mm}$, depth $50 \mu \mathrm{m}$, length 300 $\mathrm{mm}){ }^{3}$ Coverslips were coated with microspots containing 20 or $100 \mathrm{ng}$ Horm type I collagen (Nycomed Pharma, Munich, Germany) in the absence or presence of 10 pg recombinant human TF (Innovin; Dade Behring, Deerfield IL, USA). After coating, surfaces were blocked with $1 \%$ bovine serum albumin (BSA; Sigma, St. Louis MO, USA) in Hepes buffer pH 7.45 (10 mM Hepes, $136 \mathrm{mM} \mathrm{NaCl}, 2.7 \mathrm{mM} \mathrm{KCl}, 2 \mathrm{mM} \mathrm{MgCl}$ ), and used within 4 hours. Immediately before use, blood samples were pre-labelled with the membrane probe DiOC 6 (f.c. $0.5 \mu \mathrm{g} / \mathrm{mL}$, Anaspec, Reeuwijk, The Netherlands) to identify platelets (green) and with Alexa fluor (AF)647-fibrinogen (f.c. $16.5 \mu \mathrm{g} /$ $\mathrm{mL}$, Molecular Probes, Life Technologies, New York NY, USA) to detect fibrinogen and fibrin (red). Using two pulse-free micro-pumps (Model 11 Plus, 70-2212, Harvard apparatus, Holliston MA, USA) and a Y-shaped flattened mixing tube, blood samples 
in $1 \mathrm{~mL}$ plastic syringe (Becton Dickinson, Franklin Lakes $\mathrm{NJ}$, USA) were mixed with coagulation medium (Hepes buffer $\mathrm{pH} 7.45$ supplemented with $32 \mathrm{mM} \mathrm{MgCl}_{2}$ and 63

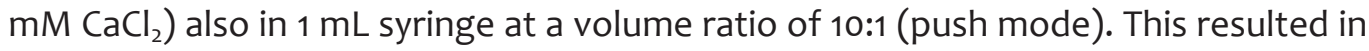
complete mixing and physiological (mM) concentrations of $\mathrm{Ca}^{2+}$ and $\mathrm{Mg}^{2+}{ }^{4}$ Where indicated, blood samples were pre-treated with $3 \mu \mathrm{g} / \mathrm{mL}$ hirudin (Refludan; Schering, Berlin, Germany) to block formed thrombin. Blood perfusion over microspots in the flow chamber was at a wall shear rate of 150 or $1,000 \mathrm{~s}^{-1}$ for at least 7 minutes.

For assessing platelet contraction in a thrombus, citrated blood samples were spiked with $3 \%$ of autologous, washed DiOC $_{6}$-labelled platelets. Where indicated, spiked blood samples were pre-treated with Gly-Pro-Arg-Pro (GPRP) or hirudin.

Two-colour confocal fluorescence microscopy and image analysis

During or after blood perfusion, two-coloured images were recorded using a fast line-scanning Zeiss LSM7 system, equipped with OPSS lasers and a $40 \times$ oil-immersion objective (numerical aperture 1.30) (Carl Zeiss, Oberkochen, Germany). Confocal fluorescence images (16 bit, $1024 \times 1024$ pixels, single channel plus overlay) were obtained at both 488 and $635 \mathrm{~nm}$ excitation. Fluorescence was detected with an ultra-sensitive CCD line camera after selection by optical emission filters of 495-555 $\mathrm{nm}$ and 665-750 nm (pinhole $1 \mathrm{AU}$ ). For time series, fluorescence images were taken at $1 \mathrm{~s}$ intervals; for $z$ stacks, fluorescence images were taken at $1 \mu \mathrm{m}$ z-steps.

Image analysis of morphometry and fluorescence was performed with Live7 Metamorph software, version 7.5.0.0 (MDS Analytical Technologies, Sunnyvale, Canada) and Image (version 1.48g: Rasband, NIH, Bethesda, MD, USA), as described elsewhere. ${ }^{5}$ For the quantification of fibrin and platelet fluorescence, images were thresholded to eliminate background. Integrated fluorescence intensity as well as percentage area covered were calculated per image. For co-localisation of twocolour fluorescence, binary images were created based on threshold to determine overlap percentages. As a standard, integrated fluorescence intensity was compared to surface area coverage of fluorescence features. Considering the overall similarity of these two analyses (Supplemental Fig. 1), for most conditions data of surface area coverage are given only.

For the quantification of (fluorescent) fibrin formation, threshold levels of fibrinogen binding to platelets was determined under conditions where coagulation was inhibited. Image intensities above this threshold were considered as originating from fibrin fibres. The presence of fluorescent fibrin fibres was always confirmed from brightfield images. Times to onset of fibrin formation were the moments at which the fluorescence threshold was passed, as confirmed by visual inspection of brightfield images.

For measurement of platelet contraction in a thrombus, time series of $\mathrm{DiOC}_{6}$ labelled images were collected during flow. Movement towards each other of labelled platelets within thrombi was analysed off-line via tracking analysis, as described. ${ }^{6}$ In 
brief, individual images of time-lapse series were thresholded to a binary images. Using the Mtrack2 plugin displacement was measured by including objects (platelets) of $<5$ pixels which were present $>60$ seconds. Objects were rejected when the displacement was $>25$ pixels s-1 (indicating noise or platelets not part of one thrombus). Calculated was per condition the average movement of platelets in time $(\mu \mathrm{m} / \mathrm{s})$.

\section{Dilution and reconstitution of whole blood samples}

Citrated blood was diluted in vitro with saline medium, consisting of $154 \mathrm{mM} \mathrm{NaCl}$, $10.8 \mathrm{mM}$ trisodium citrate, $2 \mathrm{mM} \mathrm{CaCl}$ and $2 \mathrm{mM} \mathrm{MgCl}_{2}$, in order to keep equal concentrations of free $\mathrm{Ca}^{2+}$ and $\mathrm{Mg}^{2+}$ in all diluted samples. ${ }^{2}$ Washed platelets and/ or red cells were added to diluted blood samples to restore original cell counts, as described before. ${ }^{2}$ Amounts of fluorescent labels, DiOC $_{6}$ and AF647-fibrinogen, were adjusted according to the extent of dilution and reconstitution.

\section{Measurement of thrombin generation on microspots in flow chamber}

Fluorogenic thrombin substrate Z-Gly-Gly-Arg-AMC (f.c. $0.5 \mathrm{mM}$, Thrombinoscope, Maastricht, The Netherlands) was added to blood samples where indicated, and used to measure thrombin generation on preformed thrombi under static conditions. ${ }^{7}$ Realtime fluorescence accumulation due to substrate cleavage was recorded at $447 / 30$ $\mathrm{nm}$ emission every 30 seconds for at least 5 minutes, using an inverted fluorescence microscope equipped with an Olympus $60 \times$ oil-immersion objective (numerical aperture 1.35), equipped with a $360 \mathrm{~nm}$ led cube (EVOS, Life Technologies, Bleiswijk, The Netherlands). ${ }^{5}$ Temporal analysis of fluorescence was performed using ImageJ.

Determination of microelasticity of thrombi on microspots by nanoindentation technology

Microelasticity of formed thrombi on coverslips was analysed with a recently developed Piuma nanoindenter (Optics11, Amsterdam, The Netherlands), which features a calibrated nanoindentation probe consisting of a cantilever fabricated on top of a ferruled optical fibre. Recent developments in indentation probe fabrication have resulted in probes that can measure soft (biological) materials, e.g. cartilage and vascular models. These probes measure the material hardness in the Pascal to kiloPascal range. ${ }^{8}$ In atomic force microscopy a cantilever tip is used that is repelled from the surface by minute forces. In contrast, in nanoindentation methods a spherical, cylinder or Berkovich shaped tip is used that is forced into the sample. The employed Optics11 Piuma Nanoindenter system has been validated before with biological samples. ${ }^{9-11}$

In brief, a probe with a defined cantilever of $1.39 \mathrm{~N} / \mathrm{m}$ and calibrated spherical tip of $56.5 \mu \mathrm{m}$ was gradually brought into contact with the sample surface, up to a depth of maximum $10 \%$ of the sample thickness. ${ }^{12}$ The counter-force of the sample on the tip 
results in cantilever bending, which is linear with the load on the cantilever. The result is a load-displacement curve of both loading (indenting) and unloading (retraction) of the probe. The probe, fixed on a closed-loop z-piezoelectric translator, was equipped with an integrated strain gage sensor and controlled by a closed-loop controller. From the slope of the unloading curve, the maximum load and the indentation area (tip surface area), a reduced Young's modulus (RedYM) can be calculated as a proxy measure of microelasticity. ${ }^{13}$ The modulus is the amount of force per unit area (stress), needed to reach a certain amount of deformation (unit $=\mathrm{Pa}$ ). Hence, a higher RedYM indicates that the given material is harder to deform. This measurement outcome is similar to atomic force microscopy. ${ }^{14}$

As a conservative rule of thumb, the indentation value is independent of the height and size of a sample, provided that the indentation depth is smaller than the sample radius and $<10 \%$ of the sample height. ${ }^{12,15}$ With these criterions met for the present conditions, the RedYM reflects the elastic modulus of platelet-fibrin thrombi. The attractiveness of the nanoindentation technique is that the mechanoelastic properties of a microscopic sample can directly be determined without any further sample knowledge. A drawback is the lack of visual feedback of the probe location above the thrombus sample. To overcome this, we have indented all samples (old and new conditions) 36 times in a grid-like pattern. With an optical magnifier, we further confirmed that the right spots on the sample were indented.

Coverslips with thrombi were carefully washed and incubated with Hepes buffer $\mathrm{pH} 7.45$. The diameter of the contact area of indentation tip with thrombi was $\sim 20$ $\mu \mathrm{m}^{2}$. Per microspot, 6 × 6 indentations were performed following a gridding pattern with a step-size of $50 \mu \mathrm{m}$. All indents were depth controlled $(1 \mu \mathrm{m}$ to a maximum of $5 \mu \mathrm{m}$ ). Loading and unloading times were set at 3 seconds, data were collected for 1 second per step. The unloading curves were used to calculate slopes using the compliance method of Oliver and Pharr in order to calculate the RedYM. ${ }^{13}, 16$ This is a measure of the microrigidity of the indented spot, which is inversely related to the microelasticity. A high RedYM reflects high microrigidity or stiffness of the indented sample and, hence, low microelasticity. Of the grid nanoindentations, only curves reflecting thrombi on glass were included. As negative and positive controls bare collagen surfaces and macroscopic fibrin clots were used, which showed a low and high microelasticity, respectively. Furthermore, scanning electron microscopy pictures were obtained for direct comparison of the dimensions of tip and thrombi.

\section{Scanning electron microscopy}

Thrombi were formed on microspots in a perfusion chamber, as described above, and prepared for scanning electron microscopy. In short, after disassembly of the perfusion chamber, coverslips were removed and gently washed with Hepes buffer $\mathrm{pH} 7.45$. Fixation was with $2.5 \%$ glutaraldehyde in $0.1 \mathrm{M} \mathrm{K}$-phosphate buffer $\mathrm{pH} 7.4$ at $4{ }^{\circ} \mathrm{C}$. Following a rinsing step with $0.1 \mathrm{M}$ phosphate buffer, samples were dehydrated in graded ethanol series, then critical point dried, mounted with silver paint on 
specimen stubs, and coated with gold. Thrombi were visualised with a Phillips XL30 scanning electron microscope at $10 \mathrm{kV}$ (Eindhoven, The Netherlands).

\section{Statistics}

Statistical significance of differences between two independent groups was determined using the independent samples $t$ test. Differences with $p$ values $<0.05$ were considered significant. Error bars indicate inter-individual variability, except for patient data, where the variation of independent measurements is shown; values outside reference ranges of controls (mean \pm 2 SD) were considered to be different. 
A

Platelets

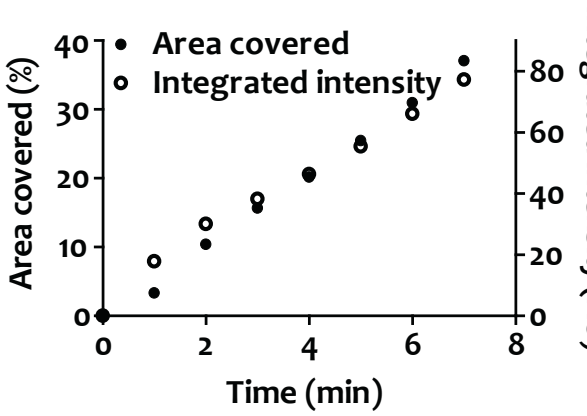

B

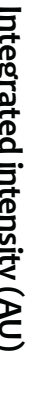

Fibrin

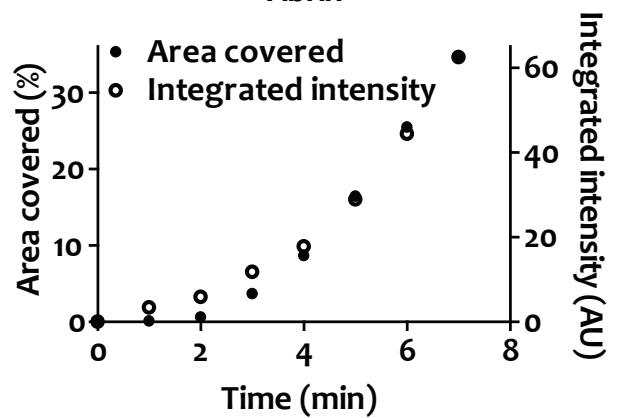

Supplemental Figure 1. Quantification of platelet-fibrin thrombus formation on collagen/TF surfaces. Citrated whole blood was perfused during recalcification over microspots with collagen (100 ng) and TF $\left(10 \mathrm{pg}\right.$ ) for 7 minutes at a wall shear rate of $1,000 \mathrm{~s}^{-1}$. Blood samples were pretreated with $\mathrm{DiOC}_{6}$ to label platelets (green) and with AF647-fibrinogen (red). (A, B) Analysis of integrated fluorescence intensity (open) and surface area coverage above background (close) from deposited platelets (A) and fibrin (B). Note high similarity of either analysis parameter.
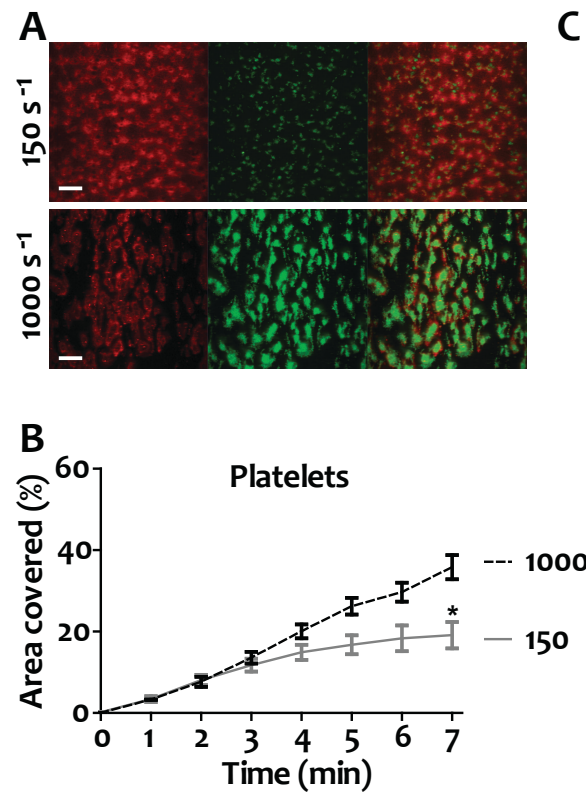

C
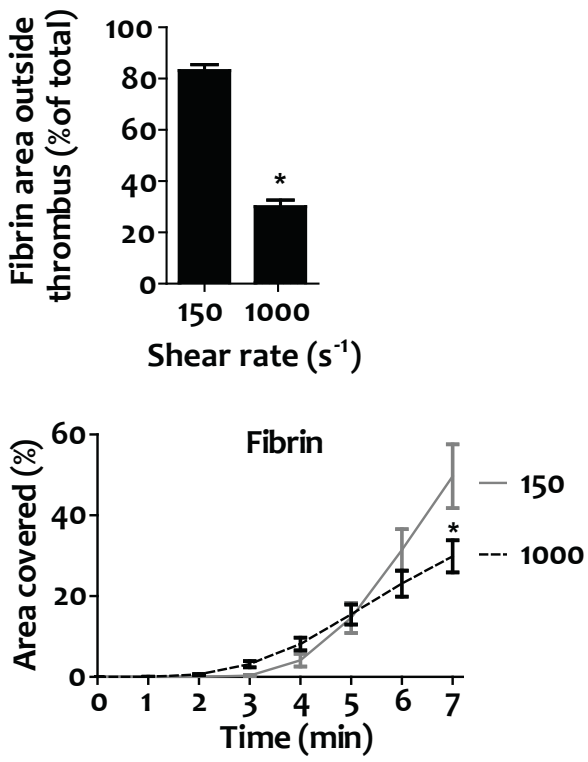

Supplemental Figure 2. Effect of wall shear rate in platelet-fibrin thrombus formation on collagentissue factor surfaces. Platelet-fibrin thrombi were formed by blood perfusion over microspots with collagen (100 ng) and TF (10 pg), as in Figure 1. Wall shear rate was 150 or 1,000 s'- . (A) Representative images after 7 minutes of flow of DiOC $_{6}$-labelled platelets (green) and AF647-fibrin(ogen) (red) (bars, 50 $\mu \mathrm{m})$. (B) Time-dependent deposition of fluorescent-labelled platelets and fibrin during blood perfusion. (C) Quantification of platelet-fibrin co-localisation at indicated shear rates. Means \pm SEM $(n=5-10),{ }^{*} p<$ 0.05 . 

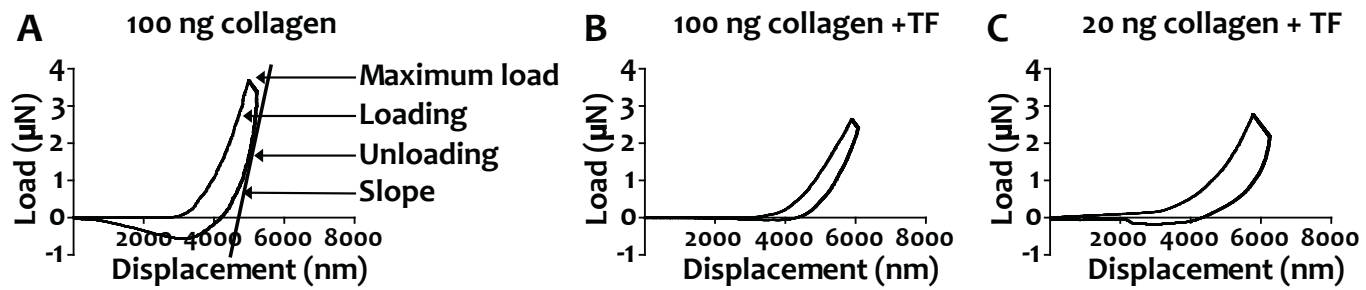

Supplemental Figure 3. Nanoindentation load-displacement curves for the assessment of microelasticity of platelet-fibrin thrombi. Platelet-fibrin thrombi were formed by blood perfusion over microspots with collagen $\pm T F$, as in Figure 1. Microspot surfaces were subjected to nanoindentation, as in Figure 4. Shown are representative load-displacement curves for thrombi formed on (A) $100 \mathrm{ng}$ collagen, (B) $100 \mathrm{ng}$ collagen plus $10 \mathrm{pg}$ TF, or (C) $20 \mathrm{ng}$ collagen plus $10 \mathrm{pg}$ TF. Indicated is the loading curve during indentation, the maximum load and the unloading curve with slope (= RedYM). The RedYM represents a measure of the microrigidity of the sample, which is inversely related to microelasticity.
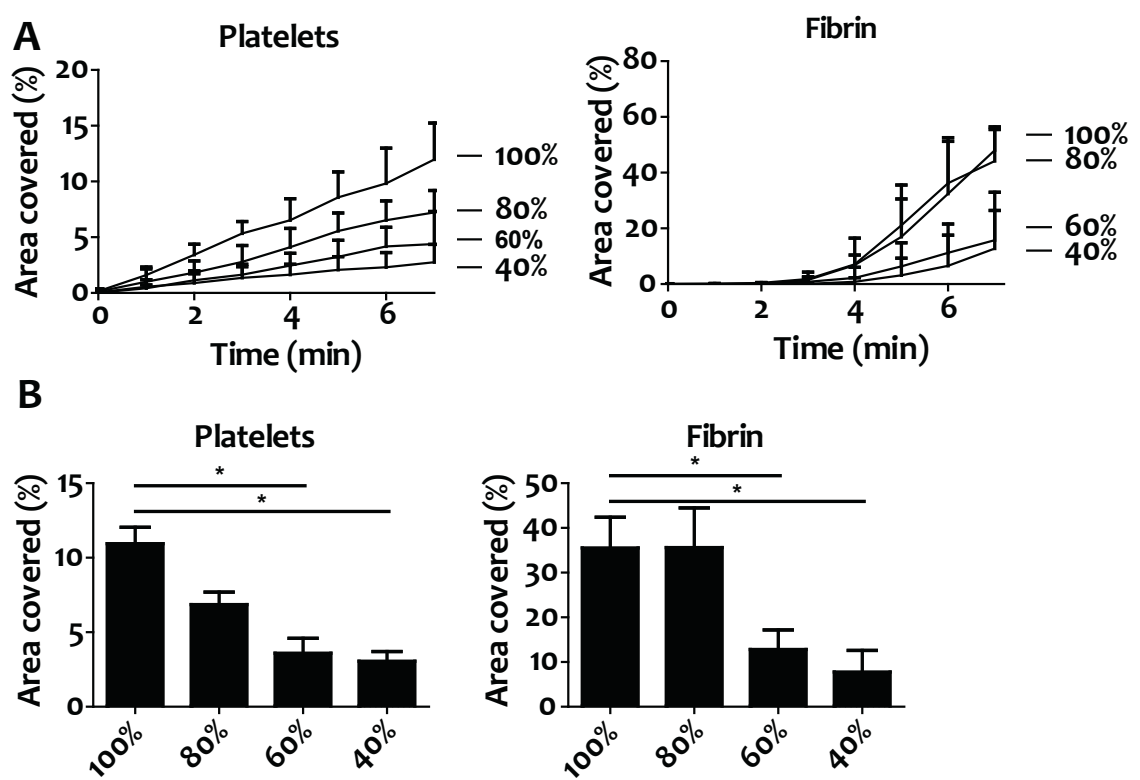

Supplemental Figure 4. Effects of blood dilution on formation of platelet-fibrin thrombus at low shear rate. Platelet-fibrin thrombi were formed by perfusion of blood over microspots with collagen (100 ng) and TF (10 pg) at low shear rate of $150 \mathrm{~s}^{-1}$ for 7 minutes. Whole blood samples were undiluted or diluted to indicated \% with saline (see Figure 5). (A) Time-dependent accumulation of fluorescence from platelets (green) and fibrin (red) during blood perfusion. (B) Fluorescence area covered by platelets and fibrin after 7 minutes of flow. Means $\pm \operatorname{SEM}(n=6) .{ }^{*} p<0.05$. 

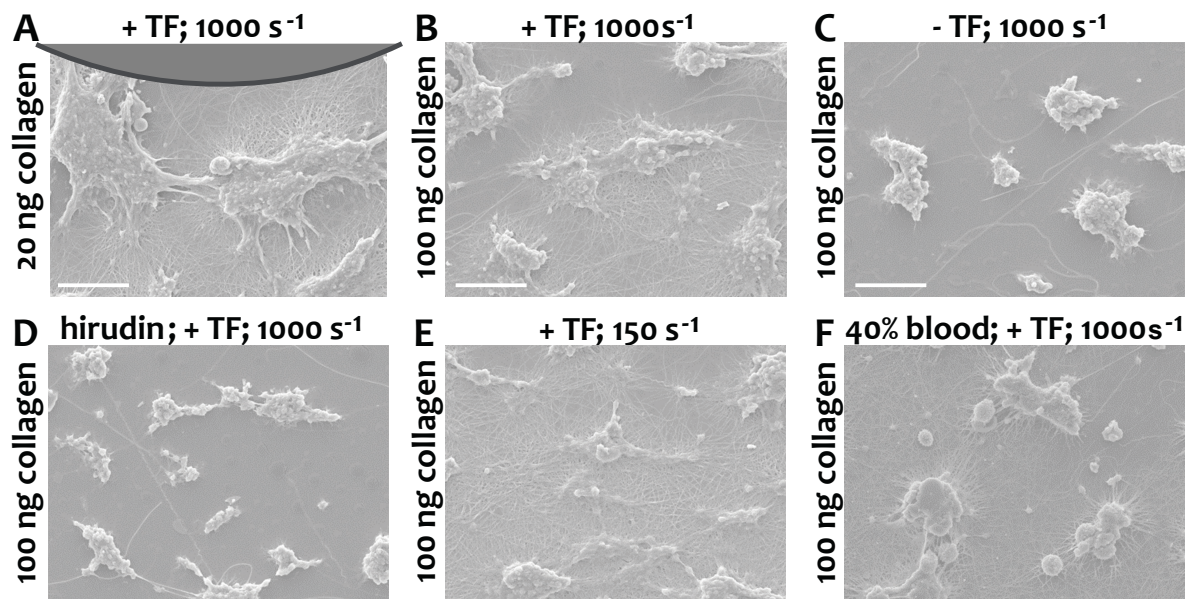

Supplemental Figure 5. Scanning electron microscopy of platelet-fibrin thrombus formed on different surfaces. Platelet-fibrin thrombi were formed by perfusion of blood over microspots with collagen $\pm \mathrm{TF}$ at shear of 150 or $1,000 \mathrm{~s}^{-1}$. Thrombi on microspots were fixed, stained and visualised with a scanning electron microscope at $10 \mathrm{kV}$. Representative $(n=3)$ images are shown from thrombi formed on: (A) 20 ng collagen + TF; (B) 100 ng collagen + TF; (C) 100 ng collagen; (D) 100 ng collagen + TF, with hirudin in blood; (E) $100 \mathrm{ng}$ collagen + TF, perfusion at $150 \mathrm{~s}^{-1}$; (F) $100 \mathrm{ng}$ collagen $+\mathrm{TF}, 40 \%$ whole blood (bars, 20 $\mu \mathrm{m})$. Upper part shows schematic representation of the spherical nanoindentation cantilever to scale.

Supplemental Table 1. Collagen-tissue factor surface determines platelet-fibrin thrombus buildup under flow. Citrated whole blood was perfused during recalcification over microspots with collagen (20 or $100 \mathrm{ng}$ ) with(out) TF (10 pg) for 7 minutes at a wall shear rate of 150 or 1,000 s-1. Blood samples were pretreated with $\mathrm{DiOC}_{6}$ to label platelets and with AF647-fibrinogen to monitor $\mathrm{f}$ ibrin formation. Twocolour confocal microscopic images were recorded in real time at a frequency of $1 \mathrm{~Hz}$. For determination of thrombus volume, confocal $z$ stacks were recorded after 7 minutes of perfusion (z-step $1 \mu \mathrm{m})$. *SAC, surface area covered; NA, not applicable due to relatively low thrombus height. Means \pm SEM $(n=6-15)$.

\begin{tabular}{|c|c|c|c|c|c|c|c|}
\hline & & \multicolumn{2}{|c|}{ Collagen 100} & \multicolumn{2}{|c|}{ Collagen $100+$ TF } & \multicolumn{2}{|c|}{ Collagen $20+$ TF } \\
\hline & & $150 \mathrm{~s}^{-1}$ & $1000 \mathrm{~s}^{-1}$ & $150 \mathrm{~s}^{-1}$ & $1000 \mathrm{~s}^{-1}$ & $150 \mathrm{~s}^{-1}$ & $1000 \mathrm{~s}^{-1}$ \\
\hline \multirow{2}{*}{$\begin{array}{l}\text { SAC* } \\
(\%)\end{array}$} & platelets & $9.04 \pm 1.7$ & $27.0 \pm 2.2$ & $19.1 \pm 3.2$ & $33.8 \pm 3.0$ & $5.62 \pm 0.7$ & $12.3 \pm 1.4$ \\
\hline & fibrin & $0.73 \pm 0.7$ & $1.19 \pm 0.1$ & $49.9 \pm 6.8$ & $29.8 \pm 4.0$ & $4.30 \pm 1.7$ & $5.76 \pm 1.1$ \\
\hline \multirow{3}{*}{$\begin{array}{l}\text { Fibrin area } \\
\text { outside } \\
\text { thrombus } \\
\text { (\% of total) }\end{array}$} & 0-10 $\mu \mathrm{m}$ (base) & $48.8 \pm 9.7$ & $36.3 \pm 8.4$ & $83.1 \pm 0.3$ & $28.8 \pm 1.9$ & $69.6 \pm 3.0$ & $9.7 \pm 2.8$ \\
\hline & $10-20 \mu \mathrm{m}$ (centre) & $54.0 \pm 18.0$ & $55.5 \pm 8.2$ & $91.4 \pm 2.1$ & $8.9 \pm 2.1$ & $55.8 \pm 5.4$ & $2.3 \pm 0.6$ \\
\hline & $>20 \mu \mathrm{m}$ (top) & $54.1 \pm 13.9$ & $75.5 \pm 14.0$ & $76.9 \pm 4.8$ & $5 \cdot 3 \pm 1.8$ & $57.5 \pm 1.3$ & $5.2 \pm 1.1$ \\
\hline \multirow{3}{*}{$\begin{array}{l}\text { Thrombus } \\
\text { volume } \\
\left(\times 10^{6} \mu \mathrm{m}^{3}\right)\end{array}$} & platelets & $0.15 \pm 0.02$ & $0.28 \pm 0.04$ & $0.16 \pm 0.02$ & $0.79 \pm 0.1$ & $0.15 \pm 0.02$ & $0.40 \pm 0.1$ \\
\hline & fibrin & $0.03 \pm 0.007$ & $0.02 \pm 0.01$ & $0.72 \pm 0.03$ & $0.76 \pm 0.2$ & $0.20 \pm 0.03$ & $0.24 \pm 0.07$ \\
\hline & total & $0.16 \pm 0.02$ & $0.28 \pm 0.04$ & $0.78 \pm 0.02$ & $1.10 \pm 0.2$ & $0.29 \pm 0.04$ & $0.46 \pm 0.1$ \\
\hline \multirow{3}{*}{$\begin{array}{l}\text { Ratio fibrin / } \\
\text { platelets }\end{array}$} & 0-10 $\mu \mathrm{m}$ (base) & $0.86 \pm 0.07$ & $0.84 \pm 0.02$ & $0.97 \pm 0.02$ & $1.21 \pm 0.1$ & $0.88 \pm 0.01$ & $0.97 \pm 0.1$ \\
\hline & $10-20 \mu \mathrm{m}$ (centre) & $1.09 \pm 0.1$ & $1.20 \pm 0.1$ & $1.07 \pm 0.02$ & $0.87 \pm 0.04$ & $0.98 \pm 0.01$ & $0.93 \pm 0.08$ \\
\hline & $>20 \mu \mathrm{m}$ (top) & NA & $N A$ & $N A$ & $1.07 \pm 0.1$ & $1.18 \pm 0.01$ & $1.27 \pm 0.1$ \\
\hline
\end{tabular}




\section{References}

1. Castoldi E, Collins PW, Williamson PL, Bevers EM. Compound heterozygosity for 2 novel TMEM16F mutations in a patient with Scott syndrome. Blood. 2011;117:4399-4400.

2. Ninivaggi M, Feijge MA, Baaten CC, Kuiper GJ, Marcus MA, Ten Cate H, Lancé MD, Heemskerk JW, Van der Meijden PE. Additive roles of platelets and fibrinogen in whole-blood fibrin clot formation upon dilution as assessed by thromboelastometry. Thromb Haemost. 2014;111:447-457.

3. Van Kruchten R, Cosemans JM, Heemskerk JW. Measurement of whole blood thrombus formation using parallel-plate flow chambers - a practical guide. Platelets. 2012;23:229-242.

4. Gilio K, Van Kruchten R, Braun A, Berna-Erro A, Feijge MA, Stegner D, Van der Meijden PE, Kuijpers MJ, Varga-Szabo D, Heemskerk JW, Nieswandt B. Roles of platelet STIM1 and Orai1 in glycoprotein VI- and thrombin-dependent procoagulant activity and thrombus formation. J Biol Chem. 2010;285:23629-23638.

5. De Witt SM, Swieringa F, Cavill R, Lamers MM, Van Kruchten R, Mastenbroek T, Baaten C, Coort S, Pugh N, Schulz A, Scharrer I, Jurk K, Zieger B, Clemetson KJ, Farndale RW, Heemskerk JW, Cosemans JM. Identification of platelet function defects by multi-parameter assessment of thrombus formation. Nat Commun. 2014;5:4257.

6. Smal I, Meijering E. Quantitative comparison of multiframe data association techniques for particle tracking in time-lapse fluorescence microscopy. Med Image Anal. 2015;24:163-189.

7. Berny MA, Munnix IC, Auger JM, Schols SE, Cosemans JM, Panizzi P, Bock PE, Watson SP, McCarty OJ, Heemskerk JW. Spatial distribution of factor Xa, thrombin, and fibrin(ogen) on thrombi at venous shear. PLoS One. 2010;5:e10415.

8. Chavan D, Van de Watering TC, Gruca G, Rector JH, Heeck K, Slaman M, Iannuzzi D. Ferrule-top nanoindenter: an optomechanical fiber sensor for nanoindentation. Rev Sci Instrum. 2012;83:115110.

9. Mattei G, Gruca G, Rijnveld N, Ahluwalia A. The nano-epsilon dot method for strain rate viscoelastic characterisation of soft biomaterials by spherical nano-indentation. J Mech Behav Biomed Mater. 2015;50:150-159.

10. Neufurth M, Wang X, Tolba E, Dorweiler B, Schroder HC, Link T, Diehl-Seifert B, Muller WE. Modular Small Diameter Vascular Grafts with Bioactive Functionalities. PLoS One. 2015;10:e0133632.

11. Wang S, Wang X, Draenert FG, Albert O, Schroder HC, Mailander V, Mitov G, Muller WE. Bioactive and biodegradable silica biomaterial for bone regeneration. Bone. 2014;67:292-304.

12. Panich N, Sun Y. Effect of penetration depth on indentation response of soft coatings on hard substrates: a finite element analysis. Surface \& Coatings Technology. 2004;182:342-350.

13. Oliver WC, Pharr GM. An improved technique for determining hardness and elastic modulus using load and displacement sensing indentation experiments. J Mater Res. 1992;7:1564-1583.

14. Chavan D, Andres D, lannuzzi D. Note: ferrule-top atomic force microscope. Il. Imaging in tapping mode and at low temperature. Rev Sci Instrum. 2011;82:046107.

15. Poon B, Rittel D, Ravichandran G. An analysis of nanoindentation in elasto-plastic solids. Int J Solids Struct. 2008;45:6399-6415.

16. Cabibbo M, Ricci P, Cecchini R, Rymuza Z, Sullivan J, Dub S, Cohen S. An international round-robin calibration protocol for nanoindentation measurements. Micron. 2012;43:215-222. 



\section{CHAPTER 7}

Rate-limiting roles of tenase complex of factors VIII and IX in platelet procoagulant activity and formation of platelet-fibrin thrombi

Swieringa F, Kuijpers MJ, Lamers MM, Van der Meijden PE, Heemskerk JW

Haematologica. 2015;100:748-56 Reprinted with permission 


\begin{abstract}
The importance of factor Xa generation in thrombus formation has not been studied extensively so far. Here, we used mice deficient in either factor VIII or factor IX to determine the role of platelet-stimulated tenase activity in the formation of plateletfibrin thrombi on collagen. With tissue factor present, deficiency in factor VIII or IX markedly suppressed thrombus growth, fibrin formation and platelet procoagulant activity (phosphatidylserine exposure). In either case, residual fibrin formation was eliminated in the absence of tissue factor. Effects of factor deficiencies were antagonised by supplementation of the missing coagulation factor. In wild-type thrombi generated under flow, phosphatidylserine-exposing platelets bound (activated) factor IX and factor X, whereas factor VIII preferentially co-localised at sites of von Willebrand factor binding. Furthermore, proteolytic activity of the generated activated factor $X$ and thrombin was confined to the sites of phosphatidylserine exposure. With blood from a haemophilia A or B patient, the formation of plateletfibrin thrombi was greatly delayed and reduced, even in the presence of high concentrations of tissue factor. A direct activated factor $\mathrm{X}$ inhibitor, rivaroxaban, added to human blood, suppressed both thrombin and fibrin formation. Together, these data point to a potent enforcement loop in thrombus formation due to factor $X$ activation, subsequent thrombin and fibrin generation, causing activated factor $\mathrm{X}$-mediated stimulation of platelet phosphatidylserine exposure. This implies that the factor VIII/factor IX-dependent stimulation of platelet procoagulant activity is a limiting factor for fibrin formation under flow conditions, even at high tissue factor concentrations.
\end{abstract}

\title{
Introduction
}

Platelet thrombus formation is a highly dynamic process that is tightly controlled by the rheological conditions and the activity of blood components at the vascular surface. The formation of a stable thrombus relies on interactions between activated platelets on the one hand, and the coagulation system generating thrombin and fibrin on the other hand. ${ }^{1-3}$ In vivo mouse models have shown that fibrin formation occurs hand-in-hand with platelet activation in the arterial and venous parts of the circulation. ${ }^{4-6}$ Furthermore, in vivo and in vitro flow studies have indicated that both the extrinsic coagulation pathway - triggered via tissue factor and factor (F) VIla -7-9 and the intrinsic pathway - triggered via FXII and resulting in FXI and FIX activation $-^{10,11}$ contribute to the formation of a stable thrombus. Tissue factor becomes exposed after vascular damage, ${ }^{12}$ but can also be recruited to a growing thrombus via leukocytes. ${ }^{13}$ Taken together, current evidence indicates that dual triggering of the coagulation system is required to generate sufficient amounts of thrombin and fibrin to stabilise the growing thrombus. It is recognised that thrombin and fibrin are generated in a resonance loop with platelet activation via the prothrombinase 
complex. ${ }^{14,15}$ However, the precise mechanism of FX activation via the tenase complex is not well understood.

Upon stimulation with strong agonists such as collagen and thrombin, platelets expose phosphatidylserine at their surface, as a consequence of $\mathrm{Ca}^{2+}$-dependent activation of phospholipid scrambling via anoctamin 6 (TMEM16F). ${ }^{16,17}$ Exposure of phosphatidylserine stimulates the procoagulant activity of platelets, by enhancing the binding and activation of coagulation factors at the platelet surface. ${ }^{1,3}$ A physiological role of phosphatidylserine exposure is evident from the observation that chelation of phosphatidylserine by annexin $A 5$ or a genetic deficiency in anoctamin 6 greatly impairs the formation of thrombi in mouse models in vivo. ${ }^{5,17}$ Earlier studies showed that phosphatidylserine-exposing platelets stimulate prothrombinase activity, by binding FVa, FXa and prothrombin, resulting in the cleavage of prothrombin into thrombin.9, 15 It has also been demonstrated that phosphatidylserine exposure is a driving force for the formation of fibrin networks on the platelet surface. ${ }^{18}$ In analogy, it has frequently been suggested, ${ }^{19-21}$ but not directly proven, that platelet phosphatidylserine exposure regulates the tenase complex, i.e. the binding of FVIIIa and FIXa to cleave FX into FXa. This is a relevant issue, since FX can also be activated by a direct interaction with the tissue factor/FVIIa complex. ${ }^{19}$ Deficiency of FVIII or FIX in humans with haemophilia A or $\mathrm{B}$, respectively, causes a mild to severe bleeding disorder. Early work, particularly using animal models, indicated that in these forms of haemophilia initial platelet plug formation (primary haemostasis) is unaffected, whereas ensuing fibrin formation (secondary haemostasis) is markedly diminished. ${ }^{22-24}$ This can be explored in test-tube experiments, suggesting that certain rates of FXa as well as thrombin generation are needed to produce a stable haemostatic platelet-fibrin clot, and that both FVIII and FIX are required for sufficient levels of FXa generation..$^{25-27}$ Indeed, in (treated) patients with haemophilia, residual thrombin generation appears to be linked to the remaining levels of FVIII or FIX. ${ }^{28}$ Mice deficient in FVIII have appeared to be valuable models for haemophilia $\mathrm{A}$, since they show prolonged bleeding times and delayed thrombus formation under flow in vivo. ${ }^{29-32}$ Similarly, mice deficient in FIX exhibit a bleeding phenotype and show reduced thrombus formation in vivo. ${ }^{33-35}$

In the present study, we aimed to establish the role of FX activation (tenase) via FVIII and FIX in the regulation of a stable, fibrin-platelet thrombus under flow conditions. In order to do so, we measured this process using blood from $\mathrm{F}^{-/}$and $\mathrm{Fg}^{-/}$ mice lacking these factors, and determined the binding of these on the thrombus. The data point to a prominent feed-forward role of locally generated FXa not only in fibrinclot formation, but also in enhancement of platelet phosphatidylserine exposure.

\section{Materials and methods}

Animals

Experiments were approved by the local animal experimental and care committee. 
$\mathrm{F}^{-1-}$ and $\mathrm{Fg}^{-/}$mice and matched wild-type mice came from the Jackson Laboratory and Charles River, respectively. All mice were bred on a $\mathrm{C} 57 \mathrm{Bl} / 6$ background. Blood was obtained by aortic puncture under anesthesia (ketamine/xylazine, Eurovet) and collected into $1 / 10$ volume of $129 \mathrm{mM}$ trisodium citrate, as described elsewhere. ${ }^{36}$

\section{Human blood preparation}

Blood was taken from healthy volunteers, a patient with haemophilia A ( $4 \%$ FVIII) and a patient with haemophilia $B(5 \%$ FIX) after their full informed consent, in accordance with the Declaration of Helsinki. Blood samples were collected by puncture of the median cubital vein into $1 / 10$ volume of $129 \mathrm{mM}$ trisodium citrate. ${ }^{15}$ In both patients, the levels of other coagulation factors were in the normal range.

\section{Labels and antibodies}

Annexin A5 labelled with fluorescein isothiocyanate (FITC) or Alexa Fluor (AF)647 was from PharmaTarget; fibrinogen labelled with Oregon Green (OG)488, AF546 or AF647 came from Invitrogen. Fluorogenic thrombin substrate peptide, Z-Gly-Gly-Arg aminomethyl coumarin (ZGGR-AMC), was from Bachem, and FXa substrate peptide Pefafluor from Pentapharm. Polyclonal AF647-labelled antibodies against human/ mouse FVIII(a) (ABIN895941) or FIX(a) (ABIN1403082) and AF647-labelled irrelevant IgG (A21240) were obtained from Antibodies Online. FITC-labelled antibody against human/mouse von Willebrand factor (VWF) came from Affinity Biologicals (GAVWFAPFTC). Human FXa was purified and labelled (active-site with $\mathrm{Na}$-[(acetylthio)acetyl]D-Phe-Pro-Arg chloromethyl ketone, followed by mild treatment with $\mathrm{NH}_{2} \mathrm{OH}$ and reaction of the thiol generated with $\mathrm{OG} 488$-5-iodoacetamide, as described. ${ }^{15,37}$ It was confirmed that the active-site labelled factor lacked protease activity, but retained normal binding properties.

\section{Whole blood flow chamber experiments}

Flow experiments were performed with a parallel-plate transparent flow chamber, containing a coverslip coated with spots of type I Horm collagen (Nycomed Pharma), blocked with Hepes buffer pH 7.45 (137 mM NaCl, 5 mM Hepes, $2.7 \mathrm{mM} \mathrm{KCl,} 2 \mathrm{mM}$ $\mathrm{MgCl}_{2}$, and $0.1 \%$ glucose), containing $1 \%$ bovine serum albumin..$^{38}$ Samples of citrateanticoagulated blood (400-1,500 $\mu \mathrm{L})$ were made to flow at defined wall shear rate, as described for murine ${ }^{39}$ and human ${ }^{15}$ blood. Coagulation was induced by co-perfusion of recalcification buffer using a dual syringe pump system (1:1 mouse: $110 \mathrm{mM} \mathrm{NaCl}$, $13.3 \mathrm{mM} \mathrm{CaCl}$, $6.7 \mathrm{mM} \mathrm{MgCl}$, $0.1 \%$ glucose and $0.1 \%$ bovine serum albumin; 1:10 human: $10 \mathrm{mM}$ Hepes, $136 \mathrm{mM} \mathrm{NaCl}, 2.7 \mathrm{mM} \mathrm{KCl}, 6.3 \mathrm{mM} \mathrm{CaCl}_{2}, 3.15 \mathrm{mM} \mathrm{MgCl}, 0.1$ $\%$ glucose and $0.1 \%$ bovine serum albumin). Where indicated, blood samples were supplemented with tissue factor (Innovin, 0.1-100 pM, Dade Behring), recombinant 
human FVIII $(2 \mathrm{U} / \mathrm{mL})$, recombinant human FIX $(2 \mathrm{U} / \mathrm{mL})$ or rivaroxaban ( 0.4 or $4 \mu \mathrm{g} / \mathrm{mL}$, all f.c.). Fluorescent probes, OG488-fibrinogen or AF647-fibrinogen ( $16 \mu \mathrm{g} / \mathrm{mL}$, f.c.), were added to the blood prior to perfusion. To detect phosphatidylserine exposure, thrombi on coverslips were post-stained with $0.5 \mu \mathrm{g} / \mathrm{mL}$ FITC-annexin A5. Coagulation factors were detected by post-staining with AF647-labelled antibodies against FVIII(a) or FIX(a) (1:20); control staining was performed with irrelevant AF647-labelled IgG at the same dilution. Where indicated, von Willebrand factor (VWF) was stained with FITC-labelled anti-VWF antibody $(20 \mu \mathrm{g} / \mathrm{mL})$, and FXa binding sites were identified with AF647-labelled FXa $(16.5 \mu \mathrm{g} / \mathrm{mL})$. Randomly captured images were analysed for surface area coverage of thrombi and fluorescent labels using Metamorph software (Molecular Devices).

Real-time measurement of thrombin and activated factor $X$ generation in flow chambers

To measure the activities of serine proteases, blood samples were preincubated with the fluorogenic thrombin substrate ZGGR-AMC or FXa substrate Pefafluor, each $0.5 \mathrm{mM}$. Thrombi were generated as above on a collagen surface, after which fluorescence accumulation from cleaved substrate was recorded under stasis, by capturing microscopic images every 30 seconds. ${ }^{15}$ Regions of interest with or without thrombi images were analysed for fluorescence intensity changes with ImageJ software (version 1.48g: Rasband, W.S, US NIH, Bethesda MD, USA).

\section{Statistical analysis}

The statistical significance of differences between groups was determined with the independent samples $t$ test, using the Statistical Package for Social Sciences (SPSS 11.0). Data are expressed as means \pm standard error of the mean (SEM). $P$ values < 0.05 are considered statistically significant.

\section{Results}

Key roles of murine factors VIII and IX in the formation of platelet-fibrin thrombi and platelet procoagulant activity in the presence of tissue factor

The formation of platelet-fibrin thrombi, triggered via the intrinsic or extrinsic pathway, can be studied efficiently in vitro by perfusion of murine or human blood over a collagen surface. ${ }^{15,39}$ In order to investigate the role of FVIII in this process, we used citrate-anticoagulated blood from $\mathrm{F}^{-}$or $\mathrm{F}^{+/+}$mice and made this flow during recalcification over collagen at an intermediate wall shear rate $\left(1,000 \mathrm{~s}^{-1}\right)$. The blood samples were supplemented with a surplus of $10 \mathrm{pM}$ tissue factor to stimulate the extrinsic pathway. With blood from wild-type $\mathrm{F}^{+/+}$mice, this resulted in the formation of large semi-occlusive thrombi after 4 minutes of perfusion (Fig. 1A). The thrombi were 


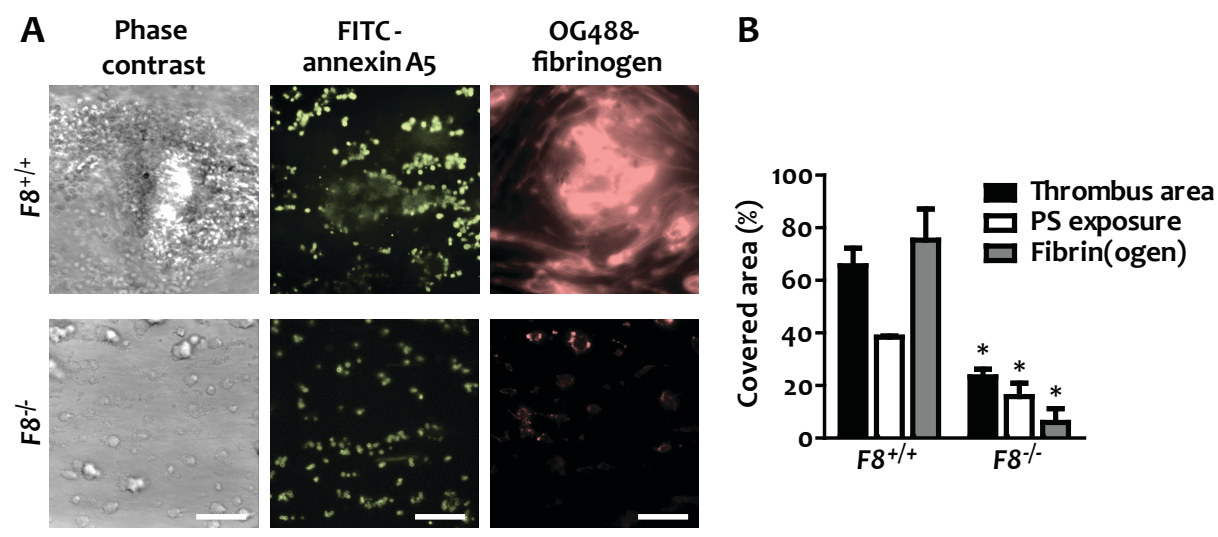

Figure 1. Role of murine FVIII in the formation of platelet-fibrin thrombi and procoagulant activity in the presence of tissue factor. Blood from wild-type mice or mice deficient in FVIII was perfused over collagen at a rate of shear $1,000 \mathrm{~s}^{-1}$ under coagulant conditions. Blood samples were preincubated with tissue factor (10 pM) and OG488-fibrinogen. Thrombi were post-stained with FITC-annexin A5 to detect phosphatidylserine (PS) exposure. (A) Representative microscopic phase contrast and fluorescence images after 4 minutes of flow (bars, $50 \mu \mathrm{m}$ ). (B) Surface area covered by platelet-fibrin thrombi (black bars), PS-exposing platelets (white bars) and fibrin(ogen) (grey bars) as determined by fluorescence studies. Means \pm SEM; $n=8-15 ;{ }^{*} p<0.05$ vs. wild-type.

composed of aggregated platelets, large patches of phosphatidylserine-exposing platelets (annexin A5 staining) and multiple fibrin fibres (fibrinogen staining). However, blood from $\mathrm{F}^{-/}$mice gave only small-sized platelet thrombi with substantially fewer phosphatidylserine-exposing platelets and limited formation of fibrin (Fig. 1A, B). As a consequence, the surface area coverage of $\mathrm{F}^{-/}$platelet-fibrin thrombi was reduced by $64 \%$, when compared to the $\mathrm{F}^{+/+}$control condition $(p=0.002)$. Fluorescent staining demonstrated a $59 \%$ reduction in phosphatidylserine-exposing platelets (annexin $A_{5}$ ) and a $92 \%$ reduction in fibrin(ogen) accumulation $(p<0.05)$.

A similar set of flow experiments over collagen was performed with blood from $\mathrm{Fg}^{-/}$and corresponding $\mathrm{Fg}^{+/+}$mice, again in the presence of tissue factor. Deficiency in FIX also resulted in a major reduction of platelet-fibrin thrombi, as well as decreased phosphatidylserine exposure and fibrin formation (Fig. 2A, B). In the case of $\mathrm{Fg}^{-/}$ blood, the surface area covered with thrombi and phosphatidylserine-exposing platelets decreased by $43 \%$ and $40 \%$, respectively, compared to the coverage when wild-type blood was used. Interestingly, this reduction was less severe than that observed with FVIII-deficient blood. Regarding thrombus size (surface area coverage) and fibrin formation, the difference between $\mathrm{F}^{-/}$and $\mathrm{Fg}^{-/}$blood was statistically significant ( $p<0.005$ and $p<0.001$, respectively). The F8 and F9 wild-type controls did not significantly differ in these parameters. Taken together, these results suggest largely overlapping functions of FVIII and FIX, but a more stringent role of FVIII, in the formation of platelet-fibrin thrombi and in the exposure of phosphatidylserine, under conditions in which exogenous tissue factor is present. 

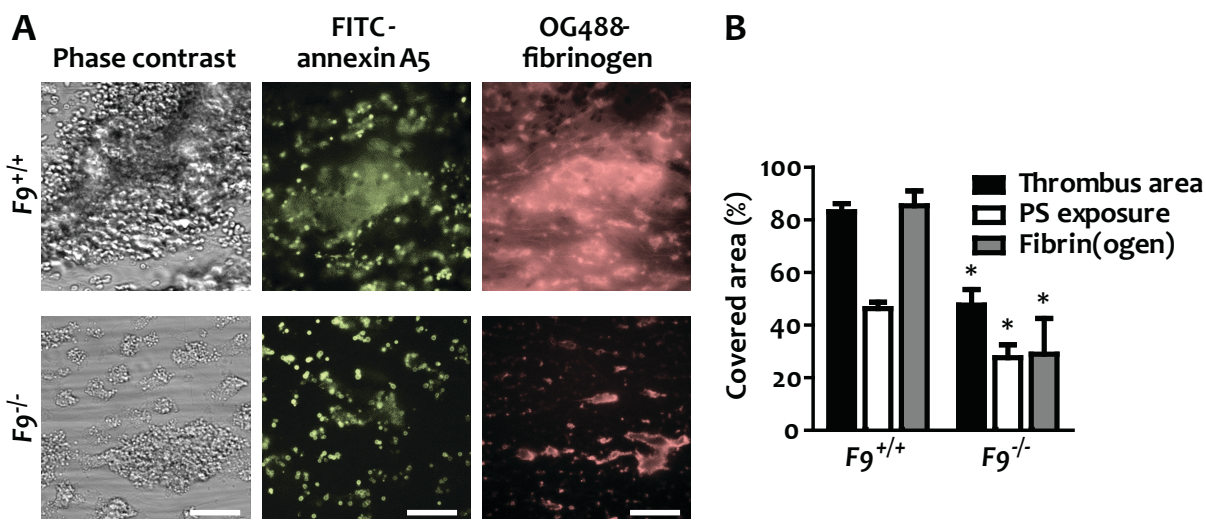

Figure 2. Role of murine FIX in the formation of platelet-fibrin thrombi and procoagulant activity in the presence of tissue factor. Blood from wild-type mice and mice deficient in FIX was perfused over collagen at a rate of shear $1,000 \mathrm{~s}^{-1}$ under coagulant conditions. Blood samples were preincubated with tissue factor (10 pM) and OG488- fibrinogen. Thrombi were then stained with FITC-annexin A5, as in Figure 1. (A) Representative microscopic phase contrast and fluorescence images (bars, $50 \mu \mathrm{m}$ ). (B) Surface area covered by platelet-fibrin thrombi (black bars), PS-exposing platelets (white bars), and fibrin(ogen) (grey bars) as determined by fluorescence studies. Means \pm SEM; $n=3-6 ;{ }^{*} p<0.05$ vs. wild-type.

Maintained roles of murine factors VIII and IX in the formation of platelet-fibrin thrombi without tissue factor

Subsequent flow studies were carried out in the absence of tissue factor to eliminate the contribution of FX activation via tissue factor/FVIla. We had previously established that in this condition the coagulation process is triggered via collagen-dependent FXII/ FXI activation. ${ }^{39}$ Flow of wild-type blood resulted in a moderately delayed, but steady formation of platelet-fibrin thrombi, again containing patches of phosphatidylserineexposing platelets and fibrin fibres (Fig. 3B, C). In this condition, flow of $\mathrm{F}^{-\%}$ or $\mathrm{Fg}^{-/ 2}$ blood (again at a shear rate of 1,000 s-1) gave small aggregates still containing procoagulant platelets, whereas fibrin fibres were absent (Fig. 3A). More specifically, with blood from $\mathrm{F}^{-/}$mice, the surface area covered by thrombi was reduced by $53 \%$, while phosphatidylserine-exposing platelets were reduced by $37 \%$ compared to the wild-type condition ( $p<0.001$ and $p<0.015$ ) (Fig. 3B, C). Similar but less stringent reductions were observed with blood from $\mathrm{Fg}^{-1-}$ mice. Addition of corn trypsin inhibitor $(20 \mu \mathrm{g} / \mathrm{mL})$, to suppress FXII activation, resulted in a further, non-significant reduction of $\sim 20 \%$ and $40 \%$ in thrombus size for $\mathrm{F}^{-/}$and $\mathrm{F}^{-/-}$blood, respectively $(p>0.10)$.

Improved formation of platelet-fibrin thrombi and platelet procoagulant activity by addition of recombinant human factor VIII or IX

To confirm that the impaired thrombus formation with knockout mice was caused by deficiency in FVIII or FIX, blood samples from $\mathrm{F}^{-/}$or $\mathrm{Fg}^{-/}$mice were treated with recombinant human FVIII or FIX, respectively. Supplementing blood from $\mathrm{F}^{-{ }^{-}}$mice 


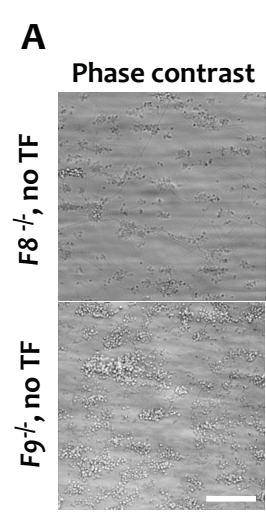

B

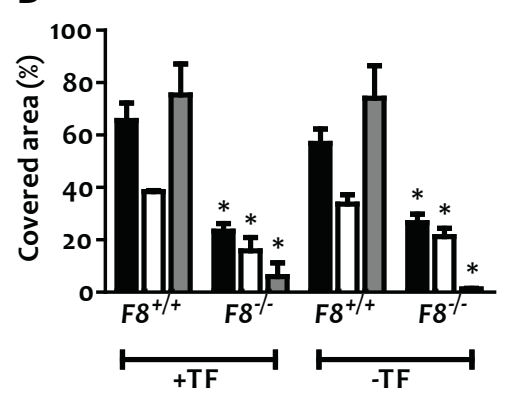

FITCannexin $\mathrm{A}_{5}$

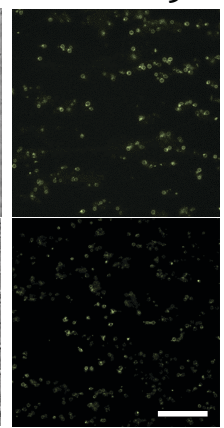

C
OG488-

fibrinogen

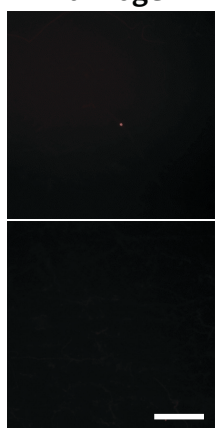

Figure 3. Retained roles of FVIII and FIX in the formation of platelet-fibrin thrombi and procoagulant activity without tissue factor. Blood from mice deficient in FVIII or FIX, or from corresponding wildtype mice was perfused over collagen under coagulant conditions. Where indicated, blood samples were preincubated with tissue factor (TF, $10 \mathrm{pM}$ ) and OG488-fibrinogen. Thrombi were then stained with FITCannexin A5 as in Figure 1. (A) Representative microscopic images after 4 minutes of flow of $\mathrm{F}^{\%}$ or F9 ${ }^{\%}$ blood in the absence of TF (bars, $50 \mu \mathrm{m}$ ). (B) Effect of TF on parameters of thrombus formation in $\mathrm{F}^{+/+}$ and $\mathrm{F}^{-/-}$mice. (C) Effect of TF on parameters of thrombus formation in $\mathrm{Fg}^{+/+}$and $\mathrm{Fg}^{-/}$mice. Means $\pm \mathrm{SEM}$; $n=3-7 ;{ }^{*} p<0.05$ vs. corresponding wild-type.

with recombinant human FVIII resulted in normalisation of the clot-formation process and a significant increase in phosphatidylserine-exposing platelets. Similarly, addition of recombinant human $\mathrm{FIX}$ to $\mathrm{F}^{-\%}$ blood resulted in the formation of large platelet-fibrin clots, which stained positively with FITC-annexin A5 (Fig. 4A, B).

\section{Tenase complex formation and activity on platelet-fibrin thrombi}

To determine the localisation of the tenase components on the murine thrombi, we first performed staining with fluorescently labelled antibodies against FVIII(a) or FIX(a). Before washing, the fluorescence of each antibody was present around the formed thrombi (Fig. 5A). However after washing, the staining for FIX(a) was exclusively detected at single platelets in a similar pattern to that seen with labelled annexin A5 staining phosphatidylserine, whereas staining for FVIII(a) was in part concentrated at fibrillar structures (Fig. 5B, C). In agreement with previous results, ${ }^{15}$ 

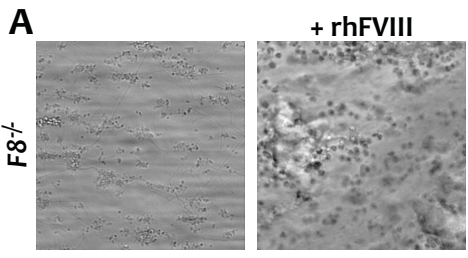

+ rhFIX
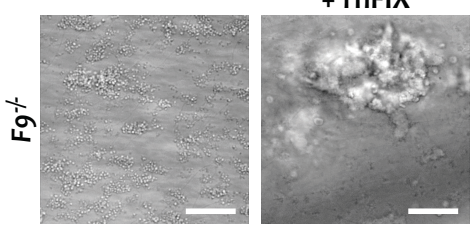

B

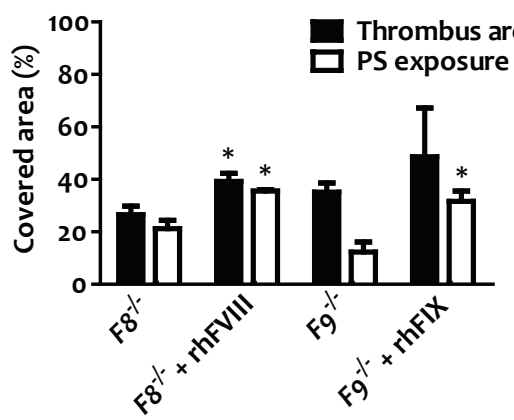

Figure 4. Restoration of thrombus formation in factor-deficient mice by supplementation of factor VIII or IX. Blood from $\mathrm{F}^{-/}$or F9 ${ }^{-/}$mice was preincubated for 10 minutes with recombinant human FVIII or FIX $(2 \mathrm{U} / \mathrm{mL})$, respectively. Samples were perfused for 4 minutes over collagen at a shear rate of $1,000 \mathrm{~s}^{-1}$, as described for Figure 1. Thrombi were then stained with FITC-annexin A5. (A) Representative microscopic phase contrast images (bars, $50 \mu \mathrm{m}$ ). (B) Surface area covered by platelet-fibrin thrombi and PS-exposing platelets. Means \pm SEM; $n=3,{ }^{*} p<0.05$.

staining of thrombi with the tenase product, labelled FXa, resulted in near complete co-localisation with annexin A5-positive platelets. Control experiments with labelled irrelevant antibodies did not result in appreciable fluorescent staining (Fig. 5D).

The concentrated staining for FVIII(a) seemed to coincide with earlier staining patterns for VWF, binding to collagen fibres. ${ }^{40}$ To investigate this further, we compared the localisation of FVIII(a) with VWF and fibrinogen by triple colour highresolution confocal microscopy using fluorescently labelled antibodies against FVIII(a) and VWF as well as labelled fibrinogen (Supplemental Fig. 1). Image inspection and overlap analysis indicated marked co-localisation of FVIII(a) with VWF in part on collagen fibres and furthermore on platelet thrombi in a similar labelling pattern as fibrin(ogen). Together, this points to FVIII(a) bound to VWF as a reservoir for tenase complex formation, in contrast to FIX(a) which seems to be bound in particular to phosphatidylserine-exposing platelets in a thrombus.

To assess the main sites of tenase activity further, murine blood was preincubated with fluorescence peptide substrates for thrombin or FXa and, once platelet-fibrin thrombi had been formed, fluorescence microscopic images were taken to determine the generation of thrombin and FXa. Image analysis under stasis indicated consistent activity of both thrombin (Fig. 6A) and FXa (Fig. 6B), occurring with highly similar kinetics, and being most prominent at areas where platelet thrombi were present. With either substrate, areas in between thrombi showed limited accumulation of fluorescence, most likely due to substrate diffusion from neighbouring areas. No fluorescence was seen at microscopic spots where no platelets (no collagen) were present (data not shown). 
A
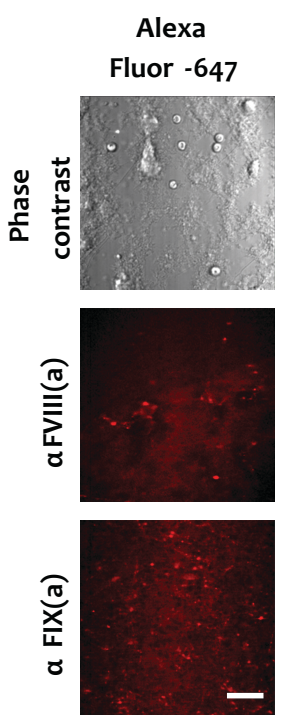

D

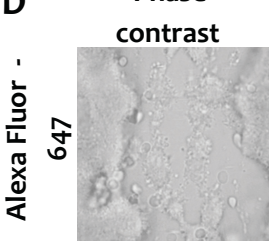

B

\section{Alexa}
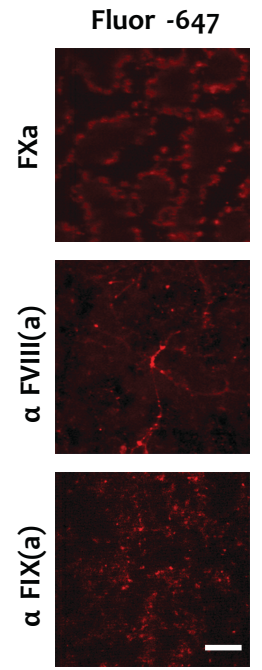

\begin{abstract}
$\lg C$
\end{abstract}

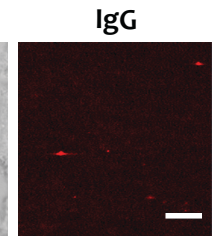

FITC -
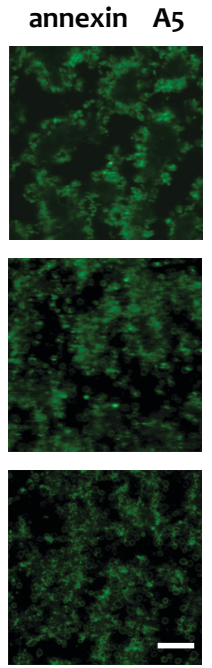

$\lg G$
C
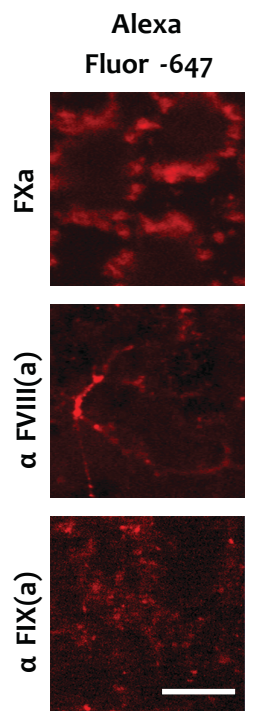

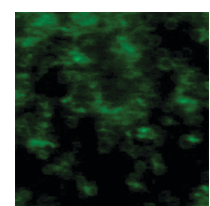

FITC annexin A5
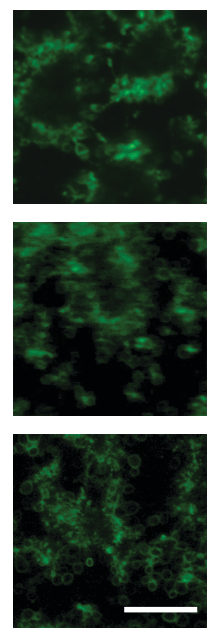

Figure 5. Localisation of (activated) FVIII, FIX and FX on murine platelet-fibrin thrombi. Blood from wildtype mice was perfused over collagen at a shear rate of 1,000 $\mathrm{s}^{-1}$ under coagulant conditions, as in Figure 1. Thrombi were then stained with FITC-annexin A5 and AF647-FXa, or AF647-labelled antibodies against FIX(a) or FVIII(a), as indicated. Representative microscopic phase contrast and fluorescence images are shown. (A) Direct antibody labelling of thrombi without rinsing. (B) Labelling of thrombi after rinsing. (C) Higher magnification images. (D) Labelling of thrombi with irrelevant AF647-antibody (bars, $20 \mu \mathrm{m}$ ).

Roles of human intrinsic and extrinsic coagulation pathways in the formation of plateletfibrin thrombi under flow

Also with human blood, in the absence of added tissue factor, fibrin clot formation on collagen under flow is known to be initiated by the intrinsic coagulation pathway. ${ }^{39}$ To determine the role of the extrinsic pathway herein, we performed perfusion experiments over collagen using whole blood with a range of tissue factor concentrations. Notably, platelet deposition over time was not affected by increasing the tissue factor concentration (Fig. 7A), indicating that this primary process is thrombin independent. However, the extent of fibrin formation was significantly enhanced after 4 minutes of perfusion with 1-100 pM tissue factor (Fig. 7B). In agreement with this, time to first fibrin formation decreased dose-dependently with 1,10 and $100 \mathrm{pM}$ of tissue factor ( $p<0.05, p<0.001$ and $p<0.0001$, respectively) (Fig. $7 \mathrm{C})$. 
A

Phase contrast
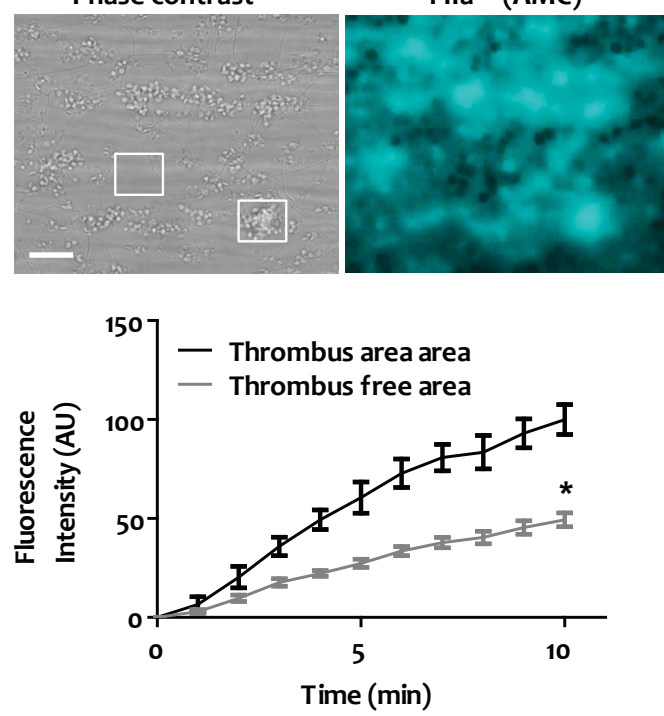

B

Phase contrast
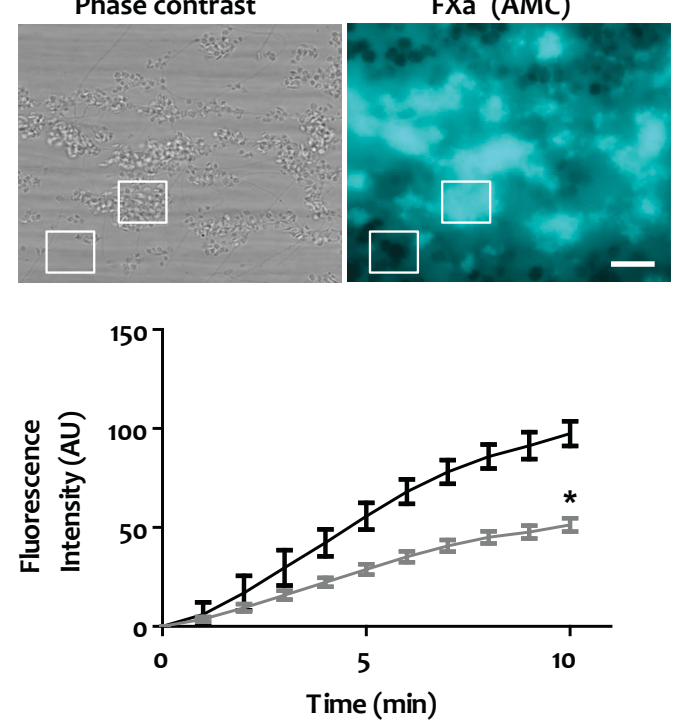

Figure 6. Generation of FXa and thrombin on preformed thrombi. Blood from wild-type mice was preincubated with (A) thrombin substrate peptide Z-GGR-AMC or (B) FXa substrate peptide Pefafluor, and perfused over collagen at a shear rate of 1,000 s'-1, as in Figure 1. After 4 minutes of thrombus formation, a time-series of microscopic fluorescence images was taken under stasis. Representative phase contrast and fluorescence images after 10 minutes (bars, $20 \mu \mathrm{m}$ ); and quantification of fluorescence intensity over time of regions-of-interest containing or not containing thrombi. Means $\pm \mathrm{SEM} ; n=8-12,{ }^{*} p<0.05$ vs. thrombi.

To assess the amplification role of FVIII and FIX at different trigger strengths, we perfused blood samples from patients with haemophilia A (4 \% FVIII) or haemophilia $\mathrm{B}(5 \% \mathrm{FIX})$ in the presence of increasing doses of tissue factor. With blood from either patient, no fibrin was formed in the absence of tissue factor over a period of 20 minutes, in comparison to the lag time of $8.6 \pm 1.9$ minutes for blood from control subjects $(p<0.0001)$. Strikingly, with blood samples from both haemophilic patients, time to fibrin formation shortened at 10 and $100 \mathrm{pM}$ tissue factor, but still remained delayed compared to that of control blood (Fig. 7D). These results indicate that the direct pathway of tissue factor/FVII(a)-induced coagulation can contribute to platelet-fibrin thrombus formation under flow, and that both FVIII and FIX enhance this process even at considerably high levels of tissue factor.

Finally, the sensitivity of this process to anticoagulant treatment was investigated by making human blood, treated with the direct FXa inhibitor rivaroxaban, ${ }^{41}$ flow over a collagen/tissue factor surface under coagulant conditions. Rivaroxaban treatment, at $4 \mu \mathrm{g} / \mathrm{mL}$, did not affect platelet deposition at the collagen surface, but greatly suppressed fibrin accumulation (Fig. 8A, B). In agreement with this, rivaroxaban strongly suppressed the cleavage of fluorescence thrombin substrate at the site of platelet thrombi (Fig. 8C). This confirmed, also for human thrombi, the presence of a potent enforcement loop of FX activation, thrombin and fibrin generation. 


\section{Discussion}

The present data point to key roles of FVIII and FIX in FX activation at the site of a platelet thrombus by supporting: (i) thrombin generation, (ii) thrombus growth and platelet phosphatidylserine exposure, and (iii) fibrin formation at the platelet surface. The likely mechanism is that tenase activity via FVIIla and FIXa, which is confined to the sites of platelet thrombi, generates FXa that directly catalyses the conversion of prothrombin into thrombin. The locally produced thrombin fulfils its multiple roles, including fibrinogen cleavage and evoking persistent intracellular signalling in platelets to stimulate phosphatidylserine exposure and enhancement of procoagulant activity. ${ }^{42}$

Early evidence for the assembly of the tenase complex of FVIIIa and FIXa came from experiments using only procoagulant phospholipid membranes. ${ }^{43}$, 44 Our localisation studies of the (activated) coagulation factors on platelet thrombi indicate that both FIX(a) and FXa concentrate on phosphatidylserine-exposing platelets, as expected, because both contain Gla-domains. However, we observed only limited and probably weak binding of FVIII(a) to phosphatidylserine-exposing platelets. Instead, FVIII(a) immuno-activity accumulated at sites of VWF localisation, coinciding with either collagen or fibrin(ogen)-labelled platelets in the thrombus. This is in agreement with the established role of VWF as a carrier of FVIII. Given that FVIII is one of the least abundant coagulation factors in plasma, these results suggest that a steady supply of (VWF-bound) FVIII to the procoagulant platelets is needed to ensure continued FXa generation. This suggestion is supported by recent evidence that locally immobilised
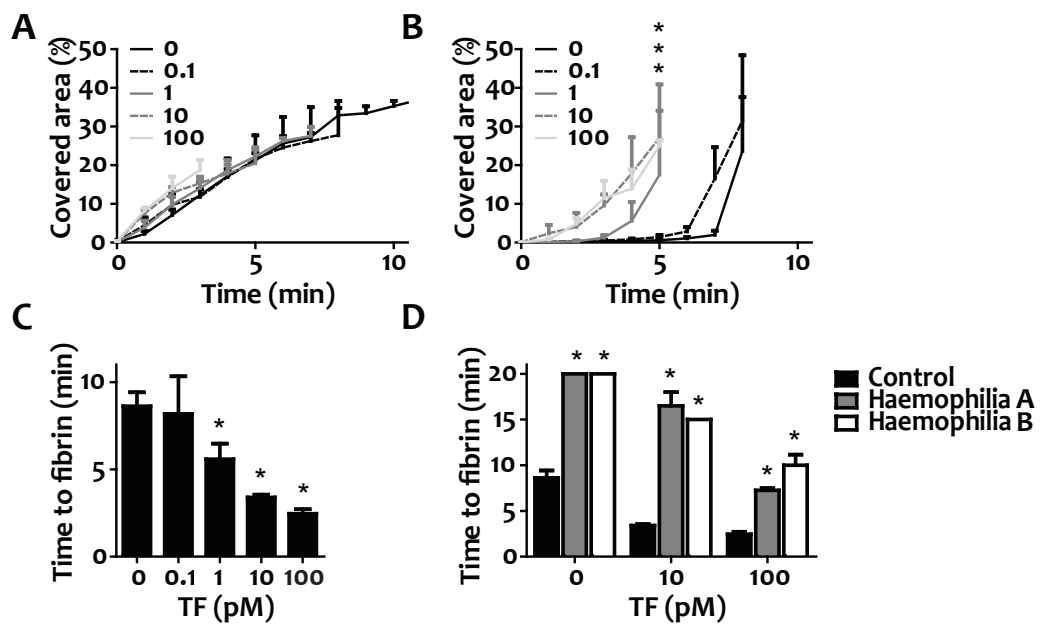

Figure 7. Maintained defective formation of platelet-fibrin thrombi with haemophilic blood at high tissue factor concentrations. Blood from normal control donors or a patient with haemophilia A (HA) or haemophilia $B(\mathrm{HB})$ was perfused over collagen at a shear rate of 1,000 s'-1 for up to 20 minutes. Blood samples were preincubated with $\mathrm{DiOC}_{6}$ to label platelets, AF647-fibrinogen and different concentrations of tissue factor (TF, 0-100 pM), as indicated. (A, B) Effect of TF concentration on surface area covered by (A) platelets and (B) fibrin(ogen) with normal blood samples. (C) Effect of TF concentration on time to first fibrin formation. (D) Prolonged time to first fibrin formation with blood from a HA or HB patient. Means \pm SEM; $n=4-6 ;{ }^{*} p<0.05$ compared to normal controls. 
A
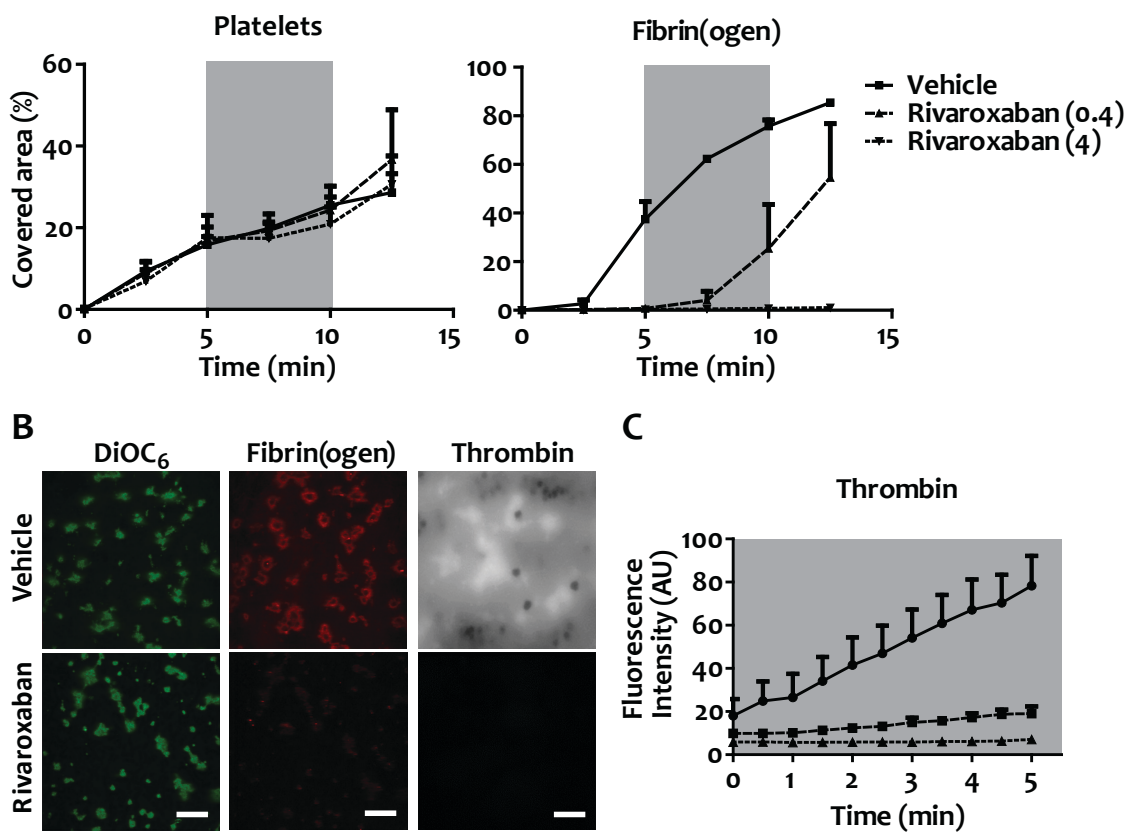

C

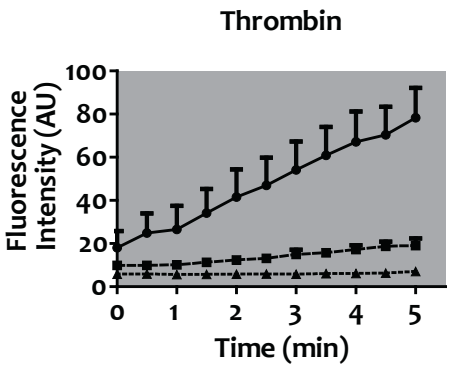

Figure 8. Effect of the FXa inhibitor rivaroxaban on the formation of platelet-fibrin thrombi. Human blood was preincubated with (A, B) OG488-fibrinogen or (B, C) thrombin substrate peptide Z-GGR-AMC and indicated concentrations of rivaroxaban $(\mu \mathrm{g} / \mathrm{mL})$, and then perfused under coagulant conditions over a collagen/tissue factor surface at a shear rate of $1,000 \mathrm{~s}^{-1}$. After 5 minutes of flow, the shear rate was reduced to $150 \mathrm{~s}^{-1}$, as indicated by the grey areas. Flow was continued at 1,000 s-1 after 5 minutes. (A) Microscopic images taken at indicated times were quantified for platelet deposition and fibrin(ogen) staining. (B) Representative fluorescence images of $\mathrm{DiOC}_{6}$-labelled thrombi, and fibrin(ogen) and accumulated $A M C$ staining. (C) Fluorescence images of $A M C$ fluorescence due to thrombin activity were quantified during 5 minutes of stasis after an initial 5 minutes of thrombus formation. Means \pm SEM; $n$ $=3$.

FVIII increases fibrin clot formation under flow, ${ }^{45}$ and that platelet-targeted FVIII can restore haemostasis in haemophilic dogs. ${ }^{46}$ Interestingly, engineered FIX variants that stimulate the coagulation process independently of FVIII appeared to reduce bleeding and normalise in vivo thrombus formation in haemophilic mice lacking FVIII. ${ }^{47}$ This latter observation is compatible with a primary role of FVIII in (soluble?) FIX activation, and the concept that thrombus-localised active FIX is sufficient for FXactivation and ensuing thrombin generation.

Based on the observation of low FVIII(a) staining of thrombi before rinsing, and relatively high, concentrated FVIII(a) staining at sites of VWF localisation after rinsing, we came to the following hypothesis. Assuming a high affinity of the FVIII(a) antibody, there appears to be low or reversible FVIII(a) binding to procoagulant platelets, i.e. at sites where FIXa is present. Likely, the FVIII(a) can be delivered to those sites from a reservoir of FVIII bound to the VWF on collagen and in the growing platelet thrombus. Earlier studies indicated that binding to VWF has no effect on the kinetics of heavy- 
chain FVIII activation, but that the cleavage of its light chain enhances the dissociation of FVIII from VWF. ${ }^{48,49}$ This is also an attractive mechanism for the presentation of FVIII from its VWF reservoir sites to procoagulant platelets during the thrombusforming process.

Whereas prothrombinase activity under flow conditions has been studied for 20 years, ${ }^{50}$ little has been reported on the regulation of FX activation, i.e. tenase activity, during thrombus formation under flow. Our data indicate that complete deficiency of murine FVIII or FIX leads to a marked impairment of the formation of fibrin-containing thrombi under flow, effects that can be restored by supplementation with human recombinant FVIII or FIX, respectively. Overall, the residual surface area covered by thrombi and fibrin(ogen) appeared to be higher for $\mathrm{Fg}^{-/}$than for $\mathrm{F}^{-/}$blood in the presence of tissue factor, suggesting that the remaining coagulant activity is higher in the absence of FIX than of FVIII. On the other hand, the data also indicate that without FIX or FVIII, procoagulant platelets and fibrin can still be formed, implying that residual amounts of thrombin are generated with tissue factor present.

In human haemophilia A, the amount of (platelet- or phospholipid-dependent) thrombin generation is considered to be a parameter related to the patient's bleeding phenotype. ${ }^{28,51}$ In this context, our findings with haemophilia A and B blood may suggest that local tenase activity at a platelet thrombus is a major regulator of fibrin clot formation and, hence, bleeding cessation. Indeed, even at high tissue factor concentrations, we still observed a prolongation of platelet-dependent fibrin formation under flow conditions. This is even more striking given the residual concentrations of FVIII ( $4 \%$ ) and FIX ( $5 \%$ ) present in the patients' blood. Jointly, these data point to a limited, supplementary role of tissue factor that still relies on both FVIII and FIX in platelet-fibrin thrombus formation under flow, likely via direct activation of FX.

Other authors have demonstrated that, in mouse models of arterial thrombosis, thrombus formation and fibrin accumulation are greatly impaired in animals deficient in either FVIII or FIX. ${ }^{30,}{ }^{35}$ Flow chamber studies in vitro also pointed to diminished fibrin clot formation with blood from mice lacking FVIII. ${ }^{32,52}$ Similar observations have been made in flow assays using blood from patients with FVIII or FIX deficiency under coagulant conditions. It was concluded that platelet deposition and fibrin formation under flow diminished depending on the residual factor activity, with fibrin formation being more sensitive to factor deficiency than platelet deposition. ${ }^{53-55}$ The present results extend these findings significantly, by indicating for the first time an enforcing role in the thrombus-forming process of procoagulant, phosphatidylserine-exposing platelets capable of FIX(a) and FXa binding.

We have unravelled a potent enforcement loop in thrombus formation, involving FX activation, subsequent thrombin and fibrin generation, and FXa-mediated stimulation of platelet phosphatidylserine exposure. The FVIII/FIX-dependent stimulation of platelet procoagulant activity seems to be a limiting factor in fibrin clotting under flow conditions. 


\section{Funding}

This research was supported by the Dutch Landsteiner Foundation for Blood Transfusion Research (1006) and the Centre for Translational Molecular Medicine (INCOAG).

\section{Authorship and disclosures}

Information on authorship, contributions, and financial \& other disclosures was provided by the authors and is available with the online version of this article at www. haematologica.org.

\section{References}

1. Heemskerk JW, Mattheij NJ, Cosemans JM. Platelet-based coagulation: different populations, different functions. J Thromb Haemost. 2013;11:2-16.

2. Jackson SP. Arterial thrombosis--insidious, unpredictable and deadly. Nat Med. 2011;17:1423-1436.

3. Mazepa M, Hoffman M, Monroe D. Superactivated platelets: thrombus regulators, thrombin generators, and potential clinical targets. Arterioscler Thromb Vasc Biol. 2013;33:1747-1752.

4. Furie B, Furie BC. In vivo thrombus formation. J Thromb Haemost. 2007;5 Suppl 1:12-17.

5. Kuijpers MJ, Munnix IC, Cosemans JM, Vlijmen BV, Reutelingsperger CP, Egbrink MO, Heemskerk JW. Key role of platelet procoagulant activity in tissue factor-and collagen-dependent thrombus formation in arterioles and venules in vivo differential sensitivity to thrombin inhibition. Microcirculation. 2008;15:269-282.

6. Von Bruhl ML, Stark K, Steinhart A, Chandraratne S, Konrad I, Lorenz M, Khandoga A, Tirniceriu A, Coletti R, Kollnberger M, Byrne RA, Laitinen I, Walch A, Brill A, Pfeiler S, Manukyan D, Braun S, Lange P, Riegger J, Ware J, Eckart A, Haidari S, Rudelius M, Schulz C, Echtler K, Brinkmann V, Schwaiger M, Preissner KT, Wagner DD, Mackman N, Engelmann B, Massberg S. Monocytes, neutrophils, and platelets cooperate to initiate and propagate venous thrombosis in mice in vivo. J Exp Med. 2012;209:819-835.

7. Dubois C, Panicot-Dubois L, Merrill-Skoloff G, Furie B, Furie BC. Glycoprotein VI-dependent and -independent pathways of thrombus formation in vivo. Blood. 2006;107:3902-3906.

8. Mangin P, Yap CL, Nonne C, Sturgeon SA, Goncalves I, Yuan Y, Schoenwaelder SM, Wright CE, Lanza F, Jackson SP. Thrombin overcomes the thrombosis defect associated with platelet GPVI/ FcRy deficiency. Blood. 2006;107:4346-4353.

9. Munnix IC, Kuijpers MJ, Auger J, Thomassen CM, Panizzi P, Van Zandvoort MA, Rosing J, Bock PE, Watson SP, Heemskerk JW. Segregation of platelet aggregatory and procoagulant microdomains in thrombus formation: regulation by transient integrin activation. Arterioscler Thromb Vasc Biol. 2007;27:2484-2490.

10. Kuijpers MJ, Van der Meijden PE, Feijge MA, Mattheij NJ, May F, Govers-Riemslag J, Meijers JC, Heemskerk JW, Renné T, Cosemans JM. Factor XII regulates the pathological process of thrombus formation on ruptured plaques. Arterioscler Thromb Vasc Biol. 2014;34:1674-1680.

11. Renné T, Pozgajova M, Gruner S, Schuh K, Pauer HU, Burfeind P, Gailani D, Nieswandt B. Defective thrombus formation in mice lacking coagulation factor XII. J Exp Med. 2005;202:271-281.

12. Day SM, Reeve JL, Pedersen B, Farris DM, Myers DD, Im M, Wakefield TW, Mackman N, Fay WP. Macrovascular thrombosis is driven by tissue factor derived primarily from the blood vessel wall. Blood. 2005;105:192-198. 
13. Gross PL, Furie BC, Merrill-Skoloff G, Chou J, Furie B. Leukocyte-versus microparticle-mediated tissue factor transfer during arteriolar thrombus development. J Leukoc Biol. 2005;78:1318-1326.

14. Beguin S, Kumar R. Thrombin, fibrin and platelets: a resonance loop in which von Willebrand factor is a necessary link. Thromb Haemost. 1997;78:590-594.

15. Berny MA, Munnix IC, Auger JM, Schols SE, Cosemans JM, Panizzi P, Bock PE, Watson SP, McCarty OJ, Heemskerk JW. Spatial distribution of factor Xa, thrombin, and fibrin(ogen) on thrombi at venous shear. PLoS One. 2010;5:e10415.

16. Van Kruchten R, Mattheij NJ, Saunders C, Feijge MA, Swieringa F, Wolfs JL, Collins PW, Heemskerk JW, Bevers EM. Both TMEM16F-dependent and TMEM16F-independent pathways contribute to phosphatidylserine exposure in platelet apoptosis and platelet activation. Blood. 2013;121:18501857.

17. Yang H, Kim A, David T, Palmer D, Jin T, Tien J, Huang F, Cheng T, Coughlin SR, Jan YN, Jan LY. TMEM16F forms a $\mathrm{Ca}^{2+}$-activated cation channel required for lipid scrambling in platelets during blood coagulation. Cell. 2012;151:111-122.

18. Cosemans JM, Schols SE, Stefanini L, De Witt S, Feijge MA, Hamulyák K, Deckmyn H, Bergmeier W, Heemskerk JW. Key role of glycoprotein Ib/V/IX and von Willebrand factor in platelet activationdependent fibrin formation at low shear flow. Blood. 2011;117:651-660.

19. Mackman N. Triggers, targets and treatments for thrombosis. Nature. 2008;451:914-918.

20. Monroe DM, Hoffman M. What does it take to make the perfect clot? Arterioscler Thromb Vasc Biol. 2006;26:41-48.

21. Zwaal RF, Schroit AJ. Pathophysiologic implications of membrane phospholipid asymmetry in blood cells. Blood. 1997;89:1121-1132.

22. Hovig T, Dodds WJ, Rowsell HC, Mustard JF. The transformation of hemostatic platelet plugs in normal and factor IX deficient dogs. Am J Pathol. 1968;53:355-373.

23. Sixma JJ, Van den Berg A. The haemostatic plug in haemophilia A: a morphological study of haemostatic plug formation in bleeding time skin wounds of patients with severe haemophilia A. Br J Haematol. 1984;58:741-753.

24. Vander Velden $\mathrm{P}$, Giles AR. A detailed morphological evaluation of the evolution of the haemostatic plug in normal, factor VII and factor VIII deficient dogs. Br J Haematol. 1988;70:345-355.

25. Hemker HC. Thrombin generation, an essential step in haemostasis and thrombosis. Haemostasis and Thrombosis, Vol I (Forbes CD, Thomas DP, Tuddenham EGD, Eds) Churchill Livingstone, UK. 1993:477-490.

26. Mann KG, Jenny RJ, Krishnaswamy S. Cofactor proteins in the assembly and expression of blood clotting enzyme complexes. Annu Rev Biochem. 1988;57:915-956.

27. Orfeo T, Brummel-Ziedins KE, Gissel M, Butenas S, Mann KG. The nature of the stable blood clot procoagulant activities. J Biol Chem. 2008;283:9776-9786.

28. Dargaud Y, Beguin S, Lienhart A, Al Dieri R, Trzeciak C, Bordet JC, Hemker HC, Negrier C. Evaluation of thrombin generating capacity in plasma from patients with haemophilia $\mathrm{A}$ and $\mathrm{B}$. Thromb Haemost. 2005;93:475-480.

29. Bi L, Lawler AM, Antonarakis SE, High KA, Gearhart JD, Kazazian HH. Targeted disruption of the mouse factor VIII gene produces a model of haemophilia A. Nat Genet. 1995;10:119-121.

30. Chauhan AK, Kisucka J, Lamb CB, Bergmeier W, Wagner DD. von Willebrand factor and factor VIII are independently required to form stable occlusive thrombi in injured veins. Blood. 2007;109:24242429.

31. Neyman M, Gewirtz J, Poncz M. Analysis of the spatial and temporal characteristics of plateletdelivered factor VIII-based clots. Blood. 2008;112:1101-1108.

32. Ogawa S, Szlam F, Dunn AL, Bolliger D, Ohnishi T, Hosokawa K, Tanaka KA. Evaluation of a novel flow chamber system to assess clot formation in factor VIII-deficient mouse and anti-factor IXatreated human blood. Haemophilia. 2012;18:926-932. 
33. Kundu RK, Sangiorgi F, Wu LY, Kurachi K, Anderson WF, Maxson R, Gordon EM. Targeted inactivation of the coagulation factor IX gene causes hemophilia B in mice. Blood. 1998;92:168-174.

34. Lin HF, Maeda N, Smithies O, Straight DL, Stafford DW. A coagulation factor IX-deficient mouse model for human hemophilia B. Blood. 1997;90:3962-3966.

35. Wang X, Cheng Q, Xu L, Feuerstein GZ, Hsu MY, Smith PL, Seiffert DA, Schumacher WA, Ogletree $M L$, Gailani D. Effects of factor IX or factor XI deficiency on ferric chloride-induced carotid artery occlusion in mice. J Thromb Haemost. 2005;3:695-702.

36. Munnix IC, Cosemans JM, Auger JM, Heemskerk JW. Platelet response heterogeneity in thrombus formation. Thromb Haemost. 2009;102:1149-1156.

37. Panizzi P, Friedrich R, Fuentes-Prior P, Kroh HK, Briggs J, Tans G, Bode W, Bock PE. Novel fluorescent prothrombin analogs as probes of staphylocoagulase-prothrombin interactions. J Biol Chem. 2006;281:1169-1178.

38. De Witt SM, Swieringa F, Cavill R, Lamers MM, Van Kruchten R, Mastenbroek T, Baaten C, Coort S, Pugh N, Schulz A, Scharrer I, Jurk K, Zieger B, Clemetson KJ, Farndale RW, Heemskerk JW, Cosemans JM. Identification of platelet function defects by multi-parameter assessment of thrombus formation. Nat Commun. 2014;5:4257.

39. Van der Meijden PE, Munnix IC, Auger JM, Govers-Riemslag JW, Cosemans JM, Kuijpers MJ, Spronk HM, Watson SP, Renné T, Heemskerk JW. Dual role of collagen in factor XII-dependent thrombus formation. Blood. 2009;114:881-890.

40. Kuijpers MJ, Schulte V, Oury C, Lindhout T, Broers J, Hoylaerts MF, Nieswandt B, Heemskerk JW. Facilitating roles of murine platelet glycoprotein $\mathrm{lb}$ and $\alpha_{11 \mathrm{~b}} \beta_{3}$ in phosphatidylserine exposure during vWF-collagen-induced thrombus formation. J Physiol. 2004;558:403-415.

41. Bauer KA. Recent progress in anticoagulant therapy: oral direct inhibitors of thrombin and factor Xa. J Thromb Haemost. 2011;9 Suppl 1:12-19.

42. Van der Meijden PE, Feijge MA, Swieringa F, Gilio K, Nergiz-Unal R, Hamulyák K, Heemskerk JW. Key role of integrin $\alpha_{1 \mathrm{lb}} \beta_{3}$ signaling to Syk kinase in tissue factor-induced thrombin generation. Cell Mol Life Sci. 2012;69:3481-3492.

43. Curtis JE, Helgerson SL, Parker ET, Lollar P. Isolation and characterization of thrombin-activated human factor VIII. J Biol Chem. 1994;269:6246-6251.

44. Lamphear BJ, Fay PJ. Factor IXa enhances reconstitution of factor VIIla from isolated A2 subunit and A1/A3-C1-C2 dimer. J Biol Chem. 1992;267:3725-3730.

45. Doi M, Sugimoto M, Matsui H, Matsunari Y, Shima M. Coagulation potential of immobilised factor VIII in flow-dependent fibrin generation on platelet surfaces. Thromb Haemost. 2013;110:316-322.

46. Du LM, Nurden P, Nurden AT, Nichols TC, Bellinger DA, Jensen ES, Haberichter SL, Merricks E, Raymer RA, Fang J, Koukouritaki SB, Jacobi PM, Hawkins TB, Cornetta K, Shi Q, Wilcox DA. Platelettargeted gene therapy with human factor VIII establishes haemostasis in dogs with haemophilia A. Nat Commun. 2013;4:2773.

47. Milanov P, Ivanciu L, Abriss D, Quade-Lyssy P, Miesbach W, Alesci S, Tonn T, Grez M, Seifried E, Schuttrumpf J. Engineered factor IX variants bypass FVIII and correct hemophilia A phenotype in mice. Blood. 2012;119:602-611.

48. Kaufman RJ, Antonarakis SE, Fay PJ. Factor VIII and hemophilia A. Hemostasis and Thrombosis (Colman RW, Hirsh J, Marder VJ, Clowes AW, George JN, Eds) Lippincott Williams \& Wilkins, Philadelphia. 2001; 135-156.

49. Hill-Eubanks DC, Lollar P. von Willebrand factor is a cofactor for thrombin-catalyzed cleavage of the factor VIII light chain. J Biol Chem. 1990;265:17854-17858.

50. Billy D, Speijer H, Willems G, Hemker HC, Lindhout T. Prothrombin activation by prothrombinase in a tubular flow reactor. J Biol Chem. 1995;270:1029-1034.

51. Wartiovaara-Kautto U, Joutsi-Korhonen L, Ilveskero S, Armstrong E, Lassila R. Platelets significantly modify procoagulant activities in haemophilia A. Haemophilia. 2011;17:743-751. 
52. Sugita C, Yamashita A, Moriguchi-Goto S, Furukoji E, Takahashi M, Harada A, Soeda T, Kitazawa T, Hattori K, Tamura S, Asada Y. Factor VIII contributes to platelet-fibrin thrombus formation via thrombin generation under low shear conditions. Thromb Res. 2009;124:601-607.

53. Colace TV, Fogarty PF, Panckeri KA, Li R, Diamond SL. Microfluidic assay of hemophilic blood clotting: distinct deficits in platelet and fibrin deposition at low factor levels. J Thromb Haemost. 2014;12:147-158.

54. Onasoga-Jarvis AA, Leiderman K, Fogelson AL, Wang M, Manco-Johnson MJ, Di Paola JA, Neeves KB. The effect of factor VIII deficiencies and replacement and bypass therapies on thrombus formation under venous flow conditions in microfluidic and computational models. PLoS One. 2013;8:e78732.

55. Weiss HJ, Turitto VT, Vicic WJ, Baumgartner HR. Fibrin formation, fibrinopeptide A release, and platelet thrombus dimensions on subendothelium exposed to flowing native blood: greater in factor XII and XI than in factor VIII and IX deficiency. Blood. 1984;63:1004-1014. 


\section{Supplemental data}

A
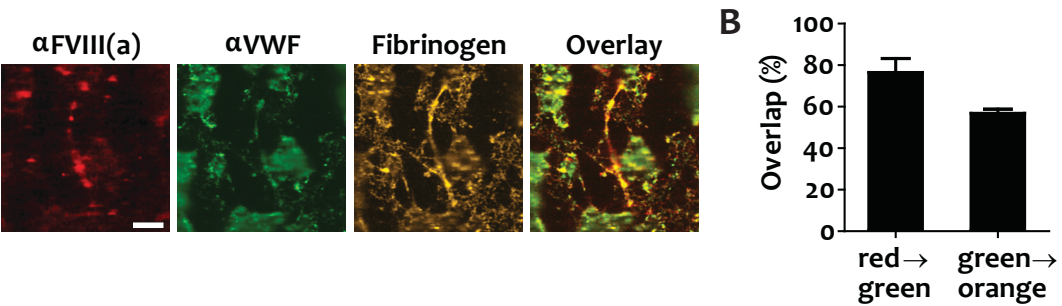

Supplemental Figure 1. Localisation of FVIII(a), VWF and fibrin(ogen) on murine platelet-fibrin thrombi. Blood from wild-type mice was perfused over collagen at 1,000 s-1 under coagulant conditions, as for Figure 1. Thrombi were post-stained with AF647-labelled antibody against FVIII(a) (red), FITC-labelled antibody against VWF (green), and AF546- labelled fibrinogen (orange), as indicated. (A) Representative confocal fluorescence images with overlay (bars, $20 \mu \mathrm{m}$ ). (B) \% Overlap analysis of red ( $\alpha$-FVIII) $\rightarrow$ green $(\alpha-V W F)$, and green $(\alpha-V W F) \rightarrow$ orange [fibrin(ogen] fluorescence (pixels thresholded above background). Means \pm SEM; $n=3$. 



\section{CHAPTER 8}

Plasminogen associates with phosphatidylserine-exposing platelets and contributes to thrombus lysis under flow

Whyte CS, Swieringa F, Mastenbroek TG, Lionikiene AS, Lancé MD, Van der Meijden PE, Heemskerk JW, Mutch NJ

Blood. 2015;125:2568-2578 Reprinted with permission 


\begin{abstract}
The interaction of plasminogen with platelets and their localisation during thrombus formation and fibrinolysis under flow are not defined. Using a novel model of whole blood thrombi, formed under flow, we examine dose-dependent fibrinolysis using fluorescence microscopy. Fibrinolysis was dependent upon flow and the balance between fibrin formation and plasminogen activation, with tissue plasminogen activator-mediated lysis being more efficient than urokinase plasminogen activatormediated lysis. Fluorescently labelled plasminogen radiates from platelet aggregates at the base of thrombi, primarily in association with fibrin. Hirudin attenuates, but does not abolish plasminogen binding, denoting the importance of fibrin. Flow cytometry revealed that stimulation of platelets with thrombin/convulxin significantly increased the plasminogen signal associated with phosphatidylserine (PS)-exposing platelets. Binding was attenuated by tirofiban and Gly-Pro-Arg-Pro amide, confirming a role for fibrin in amplifying plasminogen binding to PS-exposing platelets. Confocal microscopy revealed direct binding of plasminogen and fibrinogen to different platelet subpopulations. Binding of plasminogen and fibrinogen co-localised with PAC-1 in the centre of spread platelets. In contrast, PS-exposing platelets were PAC-1 negative, and bound plasminogen and fibrinogen in a protruding 'cap'. These data show that different subpopulations of platelets harbour plasminogen by diverse mechanisms and provide an essential scaffold for the accumulation of fibrinolytic proteins that mediate fibrinolysis under flow.
\end{abstract}

\title{
Introduction
}

Platelet accumulation is central to the haemostatic response. Platelets are activated in vivo by numerous agonists of varying potency, including thrombin, collagen, ADP, and thromboxane $A_{2}$. Platelets exhibit a nonuniform response to activation, with distinct populations forming with different surface characteristics. ${ }^{1}$ Aggregating platelets are characterised by a spherical shape, binding of fibrinogen, and expression of the active integrin $\alpha_{11 b} \beta_{3}$, and predominantly function in clot retraction. Highly activated platelets are observed on collagen fibres ${ }^{1}$ and in the core region of a thrombus nearest the vascular injury. ${ }^{2}$ These platelets are characterised by membrane exposure of phosphatidylserine (PS), a rounded balloon-like structure, sustained increase in cytosolic $\mathrm{Ca}^{2+}$, and binding of coagulation factors. ${ }^{3,4} \mathrm{PS}$-exposing platelets, also termed procoagulant platelets, substantially enhance the activity of the prothrombinase complex, ${ }^{5,6}$ and subsequent thrombin and fibrin formation. ${ }^{7}$ An additional subpopulation of platelets, termed coated platelets, are generated in response to strong dual agonist stimulation with thrombin and collagen or convulxin (CVX). ${ }^{8-10}$ Like PS-exposing platelets, coated platelets express high levels of PS and are highly procoagulant, but are differentiated by their ability to irreversibly bind a-granular proteins, such as factor $\mathrm{V}$, thrombospondin, fibrinogen, fibronectin, and 
von Willebrand factor. ${ }^{11}$ In addition to their procoagulant functions, platelets also provide a scaffold for fibrin formation and assembly of haemostatic factors.

Platelets mediate fibrinolysis by supplying a number of fibrinolytic proteins and inhibitors, including fibrinogen, plasminogen, and plasminogen activator inhibitor-1 (PAI-1). The anti-fibrinolytic function of platelet PAl-1 has been described in vitro ${ }^{12,13}$ and in vivo. ${ }^{14}$ Like PAl-1, ${ }^{15}$ plasminogen is contained in platelet $\alpha$-granules ${ }^{16,17}$ and is released upon thrombin stimulation. ${ }^{18}$ Platelet-bound plasminogen supports singlechain urokinase PA (scuPA)-mediated plasma clot lysis, ${ }^{19}$ suggesting that despite being at a low concentration ( $0.2 \mathrm{nM})$, it is a functionally active pool. Several cell receptors for plasminogen have been identified, most of which engage lysine binding sites $^{20}$ and consistent with this, can be blocked with the lysine analogue $\varepsilon A C A$, as well as antibodies directed against the fibrinogen binding site on $\alpha_{11 b} \beta_{3}{ }^{21,22}$ Binding of plasminogen to platelets is augmented by thrombin stimulation, suggesting exposure of additional sites on the activated membrane. ${ }^{21}$ When in association with the platelet surface, plasminogen assumes an open conformation that is more readily cleaved to plasmin ${ }^{19,22-26}$ and is considerably protected from inhibition by $\alpha_{2}$-antiplasmin $\left(\alpha_{2} A P\right) .{ }^{27}$, 28

Thrombi formed at high shear rates contain an abundance of platelets, whereas at low shear they are rich in erythrocytes and fibrin, ${ }^{29}$ which is aligned in the direction of flow. ${ }^{30}$ This has downstream implications in terms of thrombus stability and susceptibility to lysis ${ }^{29,31}$ and can influence penetration of cells into thrombi. Models incorporating physiological flow rates have helped define the processes governing thrombus formation..$^{29,}{ }^{32-36}$ However, studies analysing the impact of flow on fibrinolysis are limited and little is known about the distribution of plasminogen under these circumstances. In this study, we form thrombi from whole blood under physiological shear rates and manipulate the model to study fibrinolysis. Under flow plasminogen primarily associates with platelet-associated fibrin with a smaller pool found to be directly associated with platelets. Direct binding of plasminogen to platelets reveals differences in localisation depending on the subpopulation of platelets.

\section{Materials and methods}

\section{Collection of blood and preparation of platelets}

Blood was drawn from healthy controls according to the Declaration of Helsinki. In addition, remnant blood samples were obtained from 3 patients undergoing cardiothoracic surgery, before and after receiving $2 \mathrm{mg}$ of tranexamic acid to prevent bleeding complications, as a consequence of massive dilution with crystalloids and colloids. ${ }^{37}$ Protocols were approved by the local Medical Ethical Commission (METC13-4-084).

Peripheral blood was collected in $3.2 \%$ sodium citrate for thrombus formation 
or acid citrate dextrose solution A vacuettes (Greiner Bio-One) for platelet isolation. Platelets were isolated by centrifugation at $260 \times \mathrm{g}$ for 15 minutes to collect plateletrich plasma. ${ }^{35}$ Platelet-rich plasma was centrifuged at $870 \times g$ for 15 minutes and then washed by centrifugation at $870 \times g$ for 15 minutes in N-2-hydroxyethylpiperazine- $\mathrm{N}^{\text {'- }}$ 2-ethanesulfonic acid (Hepes) wash buffer (10 mM Hepes [pH 6.6], $136 \mathrm{mM} \mathrm{NaCl}, 2.7$ $\mathrm{mM} \mathrm{KCl}, 2 \mathrm{mM} \mathrm{MgCl}, 0.1 \%$ glucose, and $0.1 \%$ bovine serum albumin [BSA]) containing $0.1 \mathrm{U} / \mathrm{mL}$ apyrase (Sigma-Aldrich) and acid citrate dextrose ( $80 \mathrm{mM}$ trisodium citrate, $52 \mathrm{mM}$ citric acid, and $183 \mathrm{mM}$ glucose). Pelleted platelets were resuspended in Hepes buffer (10 mM Hepes [pH 7.45], $136 \mathrm{mM} \mathrm{NaCl}, 2.7 \mathrm{mM} \mathrm{KCl}, 2 \mathrm{mM} \mathrm{MgCl}, 0.1 \%$ glucose, and $0.1 \% \mathrm{BSA}$ ) containing $0.1 \mathrm{U} / \mathrm{mL}$ apyrase. ${ }^{35}$ Platelet counts were measured on a Siemens ADVIA 2120i Hematology System by the Haematology department, Aberdeen Royal Infirmary.

\section{Thrombus formation and lysis under flow conditions}

Citrated whole blood was perfused $\left(1,000 \mathrm{~s}^{-1}\right)$ over a glass coverslip coated with microspots of $100 \mathrm{ng}$ Horm fibrillar type I collagen (Takeda Pharmaceuticals) \pm 100 pM recombinant human tissue factor (TF) (Innovin; Dade Behring) in a transparent parallel-plate perfusion chamber. ${ }^{38}$ Blood $\pm 3 \mu \mathrm{g} / \mathrm{mL}$ hirudin was recalcified by coperfusing 1:10 (v/v) with Hepes buffer $(\mathrm{pH} 7.45)$ containing $31.5 \mathrm{mM} \mathrm{MgCl}_{2}$ and 63.2 $\mathrm{mM} \mathrm{CaCl}$ by a dual inlet mechanism. Platelets were fluorescently labelled with $0.5 \mu \mathrm{g} /$ $\mathrm{mL}$ 3',3'-dihexyloxacarbocyanine iodide ( DiOC $_{6}$ ) (AnaSpec). Fibrinogen was labelled with either Alexa Fluor (AF) 647 or Oregon Green (OG) 488 (Life Technologies, Paisley, UK) and added to whole blood at $16.7 \mu \mathrm{g} / \mathrm{mL}$ and $75 \mu \mathrm{g} / \mathrm{mL}$, respectively. ${ }^{36}$ Where indicated, thrombi were perfused with $0.8 \mu \mathrm{M}$ glu-plasminogen (Enzyme Research Laboratories) labelled with DyLight (DL) 633 (Pierce, Thermo Scientific). For fibrinolysis experiments, tissue PA (tPA) (Technoclone) or urokinase PA (uPA) (National Institute for Biological Standards and Control) (o to $75 \mathrm{nM}$ ) were added to the blood and thrombi formed for 7 minutes before perfusing for 8 minutes with buffer 1 (Hepes buffer, $\mathrm{pH}$ 7.45), at indicated shear rate. In some experiments, thrombi were perfused with whole blood \pm heparin $(3 \mu \mathrm{g} / \mathrm{mL})$ containing tPA $(75 \mathrm{nM})$. If lysis was incomplete at 8 minutes, perfusion was continued for up to 25 minutes (total time) in buffer 2 (Hepes buffer, pH 7.45 containing tPA or uPA at indicated concentrations). Fluorescence time course images and $z$ stacks were generated using a confocal Zeiss Live 7 laser scanning microscope 60 X/1.4 NA oil immersion objective. Spatial and temporal analysis of fluorescence changes, as well as overlap coefficients $R$ were determined using Zeiss Live 7 software and Image J (version 1.48g: Rasband, W.S, US NIH, Bethesda MD, USA).

Plasmin activity assays

Washed platelets $\left(5 \times 10^{8}\right.$ platelets $\left./ \mathrm{mL}\right)$ in Hepes buffer, $\mathrm{pH} 7.45$ were stimulated 
for 45 minutes with $100 \mathrm{nM}$ thrombin $+100 \mathrm{ng} / \mathrm{mL} \mathrm{CVX}$ (Pentapharm or purified to homogeneity from the venom of Crotalus durissus terrificus ${ }^{39}$ ) or $15 \mu \mathrm{M}$ thrombin receptor activator peptide 6 (TRAP-6) (Sigma-Aldrich) + CVX in the presence of $2 \mathrm{mM}$ $\mathrm{CaCl}_{2} \cdot{ }^{40}$ Following activation, $6 \times 10^{7}$ platelets $/ \mathrm{mL}$ were removed and $0.3 \mu \mathrm{g} / \mathrm{mL}$ hirudin added to prevent substrate cleavage by thrombin. Plasmin generation was measured $\pm 1 \mathrm{nM}$ tPA or uPA using $0.35 \mathrm{mM}$ D-Val-Leu-Lys-7-amido-4-methylcoumarin (SigmaAldrich). Fluorescence release (excitation $360 / 40 \mathrm{~nm}$, emission $460 / 40 \mathrm{~nm}$ ) was detected by continuous measurement in a BioTek FLx800 fluorescence microplate reader at $37^{\circ} \mathrm{C}$.

For a different set of experiments, effluents were collected during tPA-mediated thrombus lysis and immediately centrifuged to collect cell-free serum. Samples were snap-frozen in liquid nitrogen and subsequently analysed for plasmin using $0.5 \mathrm{mM}$ S2251 (Quadratech) after being diluted 7-fold in TBST (150 mM NaCl, $10 \mathrm{mM}$ Tris pH 7.4 and $0.01 \%$ Tween 20). Readings were taken at $405 \mathrm{~nm}$ every $30 \mathrm{~s}$ at $37^{\circ} \mathrm{C}$ in a BioTech ELx 808 plate reader. The concentration was determined against commercial plasmin (Enzyme Research Laboratories) using the average $\mathrm{A}_{405} / \mathrm{min}$. Hirudin $(3 \mu \mathrm{g} / \mathrm{mL}$; Merck Millipore) was included in replicate wells to check for cleavage of S2251 by residual thrombin but no differences were observed.

\section{Flow cytometry}

Washed platelets at $2 \times 10^{8}$ platelets $/ \mathrm{mL}$ in Hepes buffer, $\mathrm{pH} 7.45$, were stimulated in the presence of $2 \mathrm{mM} \mathrm{CaCl}$ with $100 \mathrm{nM}$ thrombin $\pm 100 \mathrm{ng} / \mathrm{mL} \mathrm{CVX}$, or $15 \mu M$ TRAP- 6 and $100 \mathrm{ng} / \mathrm{mL} \mathrm{CVX} \pm 1 \mu \mathrm{g} / \mathrm{mL}$ tirofiban, or $5 \mathrm{mM}$ Gly-Pro-Arg-Pro amide (GPRP) (SigmaAldrich), or Gly-Pro-Pro-Pro (Severn Biotech) as a negative control. After 40 minutes, $0.27 \mu \mathrm{M}$ plasminogen-DL633 was added before the addition of annexin A5-fluorescein isothiocyanate (FITC) (1:20) (BD Biosciences). Binding of plasminogen-DL633 was measured using an LSR II flow cytometer (Becton Dickinson). A minimum of 10,000 events were collected. Data analysis was performed using FloJo software (Tree Star).

\section{Fluorescent confocal microscopy}

Washed platelets at $0.5 \times 10^{8}$ platelets $/ \mathrm{mL}$ in Hepes buffer, $\mathrm{pH} 7.45(1 \% \mathrm{BSA})$ were adhered to $\mu$-Ibidi $I^{0.4}$ coated with $0.6 \mu$ g equine tendon type I collagen (American Biochemical Pharmaceuticals), \pm 3 pmol thrombin or $0.45 \mathrm{nmol}$ TRAP- 6 , and \pm 1 $\mu \mathrm{g} / \mathrm{mL}$ tirofiban or $5 \mathrm{mM}$ GPRP. In some cases, rabbit anti-human PAl-1 antibody ${ }^{41}$ labelled with DyLight 488 (1/20 dilution) or mouse anti-human monoclonal PAC-1 FITC antibody (1:20 dilution) (Becton Dickinson) were included. After stimulation for 40 minutes, $0.8 \mu \mathrm{M}$ plasminogen-DL633 or $16.7 \mu \mathrm{g} / \mathrm{mL}$ fibrinogen-AF647 were added. Where indicated, annexin A5-FITC (1:20 dilution) or annexin A5-AF647 (1:20 dilution) (Life Technologies) and $2 \mathrm{mM} \mathrm{CaCl}_{2}$ were included. Images were recorded on Zeiss 710 laser scanning confocal microscope with $a \times 631.40$ oil immersion objective using 
Zeiss Zen 2012 software. Analysis was performed on Bitplane's Imaris $\times 64$ software.

\section{Western blotting}

Fibrinogenolysis by tPA was analysed by incubating normal pooled human plasma \pm 10 or $75 \mathrm{nM}$ tPA for 30 minutes at $37^{\circ} \mathrm{C}$ and collected in reducing sample buffer (Life Technologies). Samples were run on 4-12\% polyacrylamide bis-Tris NuPAGE gels with MOPS running buffer (Life Technologies) under reducing conditions. Purified human plasminogen-free fibrinogen (200 ng, Enzyme Research Laboratories) was included as a positive control. Proteins were transferred to polyvinyl difluoride membrane and immunoblotted with a horse radish peroxidase conjugated sheep anti-human fibrinogen antibody (1:5,000 dilution; Enzyme Research Laboratories). Proteins were detected using ECL (Thermo Fisher-Scientific) with a UVP Biospectrum 810 imaging system and analysed with UVP VisionWorks LS Image Aquisition and Analysis software.

\section{Statistical analysis}

Statistical analysis was performed in GraphPad Prism 5.04 using one-way analysis of variance with Bonferroni post hoc test with the exception of the thrombus flow experiments, which were analysed using Student $t$ test (two-tailed). $P<0.05$ was considered to be significant. Results are represented by the mean \pm standard deviation (SD) or \pm standard error of the mean (SEM).

\section{Results}

Visualisation of fibrinolysis under high shear flow conditions

A whole blood flow model was developed to visualise fibrinolysis under a physiological shear rate $\left(1,000 \mathrm{~s}^{-1}\right)$. Thrombi consisting of platelets and fibrin(ogen) were formed for 7 minutes on collagen + TF microspot surfaces \pm tPA or UPA ( 0 to $75 \mathrm{nM}$ ). Fibrin formation was monitored by fluorescence microscopy as accumulation of fibrin(ogen)AF647 (Supplemental Video 1, available on the Blood Web site). Thrombi (nonocclusive) were subsequently perfused with buffer to monitor lysis in real time. In the absence of PA fibrin(ogen), coverage remained relatively unchanged for the duration of the experiment (Fig. 1A). Dose-dependent fibrinolysis was visualised in thrombi containing either TPA or UPA (Fig. 1B, C and Supplemental Video 2). Comparable 50 $\%$ lysis times were achieved with $30 \mathrm{nM}$ tPA and $75 \mathrm{nM}$ uPA ( $4.5 \pm 0.6$ minutes and $7.3 \pm 1.5$ minutes, respectively (Fig. $1 B, C$ ). The lower tPA concentration required was consistent with its known fibrin specificity. Analysis of effluents, collected during tPA-mediated fibrinolysis, revealed substantial plasmin activity at $75 \mathrm{nM}$ but minimal activity at $10 \mathrm{nM}$ or in its absence (Supplemental Fig. $1 \mathrm{~A}$ ). The addition of PAs to thrombi post formation (Fig. 1A) did not achieve lysis within the experimental time frame 

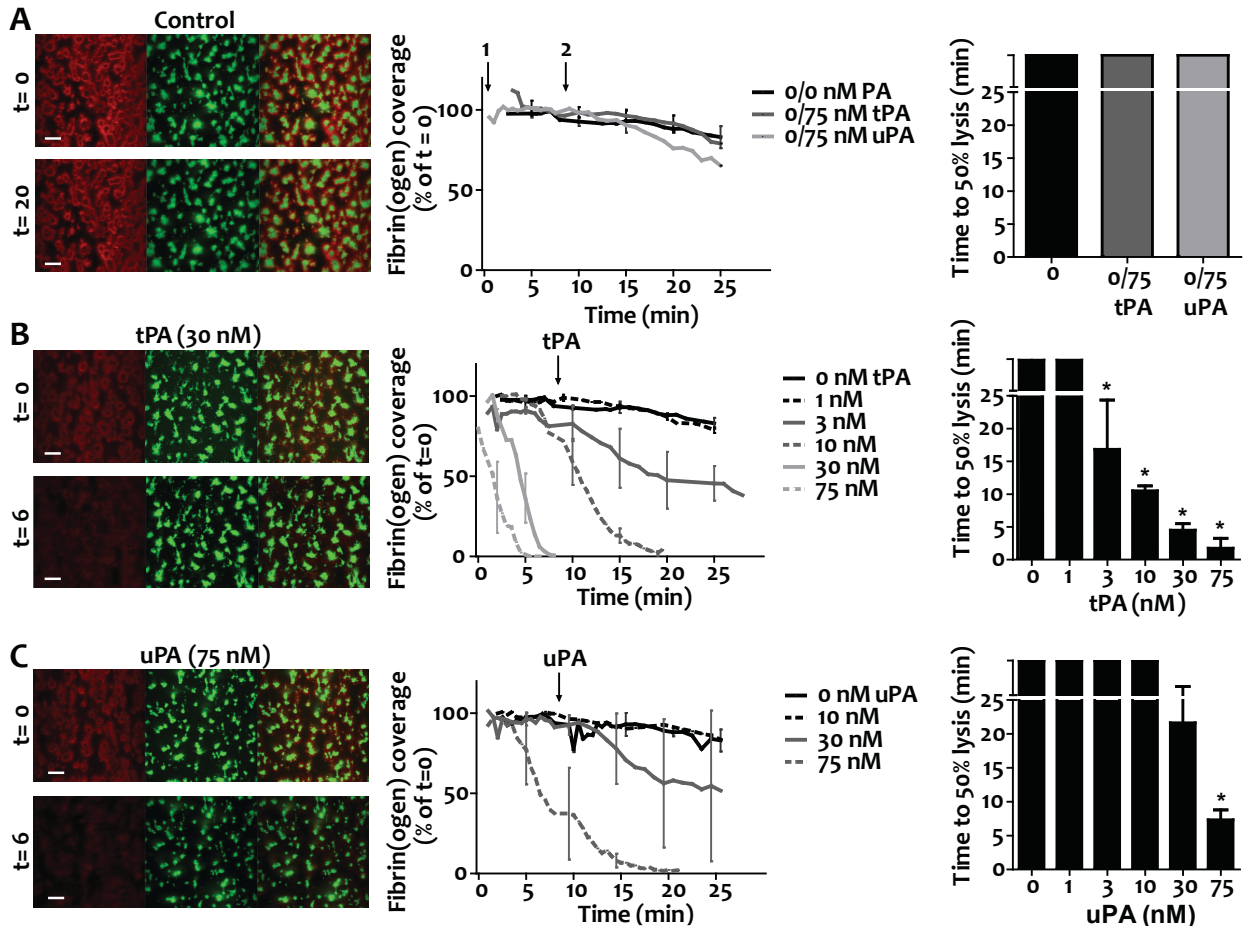

Figure 1. Concentration-dependent effect of plasminogen activators on fibrin degradation of thrombi under flow. Platelet-fibrin thrombi were formed by perfusion $\left(1,000 \mathrm{~s}^{-1}\right)$ of whole blood for 7 minutes over collagen/TF in the absence or presence of tPA or UPA (o nM to $75 \mathrm{nM}$ ). Blood samples were preincubated with $\mathrm{DiOC}_{6}(0.5 \mu \mathrm{g} / \mathrm{mL})$ to label platelets and fibrinogen-AF647 $(16.7 \mu \mathrm{g} / \mathrm{mL})$. The fluorescent thrombi were perfused $(t=0$ ) with buffer 1 (Hepes buffer, $\mathrm{pH} 7.45$ ) for 8 minutes, followed by buffer 2 (Hepes buffer, pH 7.45 with the indicated concentration of tPA or UPA) for up to 28 minutes. Shown are representative images of thrombi labelled for fibrin(ogen) (red), platelets (green), and label overlay (yellow) (left panels; bars, $50 \mu \mathrm{m}$ ). Also shown is dose-dependent fibrinolysis in time, expressed as fibrin(ogen)-AF647 surface coverage (\% of $t=0, \pm S E M$ ) (middle panels), and time to $50 \%$ lysis (right panels). (A) Absence of plasminogen activators, light bars represent controls where $75 \mathrm{nM}$ tPA or uPA were present in buffer 2 only and not during thrombus formation. (B) tPA-mediated fibrinolysis and (C) uPA-mediated fibrinolysis. Data represent means \pm SEM, * $p<0.05$ vs no tPA or uPA, $n \geq 3$.

(up to 25 minutes). Interestingly, maximum fibrinogen coverage post thrombus formation was reduced in the presence of $75 \mathrm{nM}$ tPA ( $51 \pm 3 \%$ vs $75 \pm 1 \%$ in the absence of tPA), reflecting concurrent fibrinolysis during thrombus formation. The change in fibrinogen coverage could not be explained by fibrinogenolysis, as minimal degradation was observed following incubation of tPA in plasma (Supplemental Fig. 1B). In contrast, surface coverage of $\mathrm{DiOC}_{6}$-labelled platelets was unaltered by tPA or uPA (Supplemental Fig. 1C, D). Furthermore, DiOC 6 -labelled platelet volume was investigated using confocal z stacks (16-bit images of $1024 \times 1024$ pixels; $336 \times 336 \mu \mathrm{m}$; stack distance $0.5 \mu \mathrm{m} ; 100$ slices). ${ }^{42}$ No difference was noted in total platelet volume \pm $75 \mathrm{nM}$ tPA (311 $\pm 145 \mu \mathrm{m}^{3}$ vs $267 \pm 115 \mu \mathrm{m}^{3}$ per field, $\left.p=0.43, n=4\right)$. 

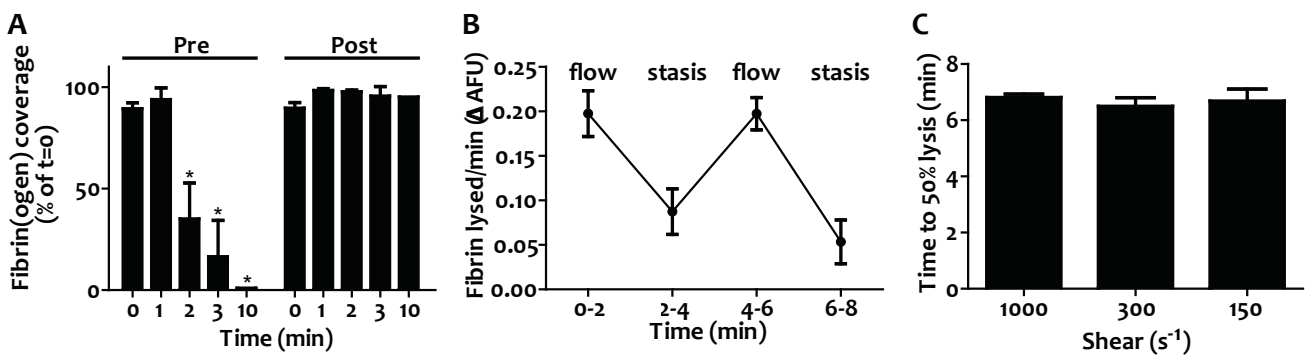

D

$\mathbf{t}=\mathbf{0}$

$\mathbf{t}=\mathbf{3}$
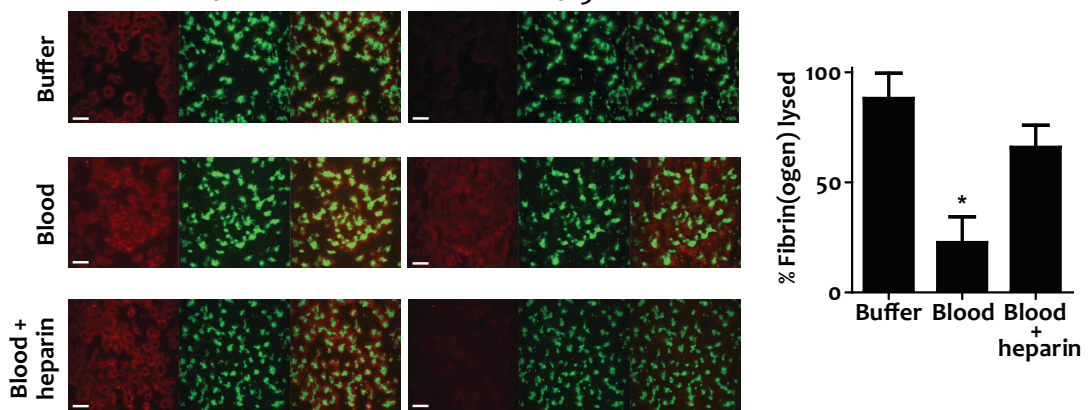

Figure 2. Fibrinolysis under physiological flow conditions. (A) Thrombi were formed from blood samples obtained from cardiothoracic patients pre- and post-tranexamic acid treatment ( $2 \mathrm{mg})$. Blood was preincubated with $\operatorname{DiOC}_{6}(0.5 \mu \mathrm{g} / \mathrm{mL})$ to label platelets and fibrinogen-AF647 $(16.7 \mu \mathrm{g} / \mathrm{mL})$, and perfused $\left(1,000 \mathrm{~s}^{-1}\right)$ over a collagen/TF-coated surface for 7 minutes in the presence of $75 \mathrm{nM}$ tPA. Hepes buffer, $\mathrm{pH} 7.45(\mathrm{t}=0)$ was then allowed to perfuse through thrombi at 1,000 s-1. The fluorescent thrombi were lysed by perfusion ( $t=0$ ) with buffer 1 (Hepes buffer, $\mathrm{pH} 7.45$ ) for 10 minutes. Quantification of surface area covered with fibrin(ogen) as compared with $t=0$. Data represent means $\pm S D, n=3$. (B) Thrombi from blood of normal individuals were formed as described for (A) except with inclusion of 10 $\mathrm{nM}$ tPA, and during the lysis stage flow was alternated between 1,000 $\mathrm{s}^{-1}$ and stasis every 2 minutes. (C) Thrombi were formed as for $(B)$ before perfusing at $1,000 \mathrm{~s}^{-1}, 300 \mathrm{~s}^{-1}$, or $150 \mathrm{~s}^{-1}(t=0)$ with buffer 1 (Hepes buffer, $\mathrm{pH}$ 7.45) for 8 minutes, followed by buffer 2 (Hepes buffer, pH 7.45 with 10 nM tPA) until lysis was complete. Quantification is shown as time to $50 \%$ lysis (mean \pm SD). (D) Thrombi were formed as above in the presence of $75 \mathrm{nM}$ tPA. After formation $(t=0)$, thrombi were subsequently perfused with Hepes buffer, $\mathrm{pH}$ 7.45, whole blood, or heparinised whole blood containing tPA (75 nM) for up to 20 minutes. Representative images of thrombi labelled for fibrin(ogen) (red), platelets (green), and overlay (yellow) (bars, $50 \mu \mathrm{m}$ ). Quantification shown of percentage of fibrin(ogen) lysed based on initial surface coverage from 0 to 3 minutes. AFU, average fluorescence units. Data represent means $\pm S E M, n \geq 3,{ }^{*} p<0.05$.

Differences in fibrinolysis were noted in cardiothoracic surgery patients treated with $2 \mathrm{mg}$ of tranexamic acid, a lysine analogue that inhibits plasminogen activation. Thrombi formed prior to tranexamic acid infusion lysed to a similar degree as healthy controls (75 nM tPA; $50 \%$ lysis times were $1.5 \pm 0.3$ minutes vs $1.75 \pm 1.5$ minutes, respectively). However, thrombi formed posttreatment did not show any appreciable lysis during the time frame (Fig. 2A).

The impact of flow on tPA-mediated lysis of thrombi was investigated by alternating between a high shear rate of $1,000 \mathrm{~s}^{-1}$ and stasis conditions. Lysis, quantified as change in fibrin(ogen) fluorescence over time, was reduced twofold during stasis (Fig 2B), reflecting the contribution of flow to fibrinolysis. When shear rates were 
altered during fibrinolysis, no differences in time to $50 \%$ lysis were observed (Fig. 2C), suggesting that flow itself is the essential parameter rather than shear rate.

The described model was manipulated to visualise both thrombus formation and lysis in the presence of whole blood. Thrombi were perfused with buffer or recalcified citrated-whole blood containing $75 \mathrm{nM}$ tPA. Complete lysis was visualised at around 3 minutes with $75 \mathrm{nM}$ tPA in buffer, but was substantially delayed in whole blood (Fig. 2D). Perfusing thrombi with heparinised blood containing $75 \mathrm{nM}$ tPA achieved a comparable level of lysis to that observed in buffer (Fig. 2D). These data indicate that in the presence of an anticoagulant to inhibit ongoing thrombin generation, ${ }^{43}$ and thus fibrin formation, fibrinolysis is more efficient.

\section{Localisation of plasminogen within thrombi}

Plasminogen activation and fibrinolysis are inextricably linked. We therefore investigated the distribution of plasminogen within thrombi under physiological flow rates. Two pools of plasminogen were detected (Fig. 3A); the larger pool primarily co-localised with platelet-associated fibrin(ogen), whereas a smaller pool was directly associated with platelets. Overlap coefficients $(R)$ were high $(0.89 \pm 0.02)$ for plasminogen and fibrinogen, and lower $(0.45 \pm 0.01)$ for plasminogen and platelets (Fig. 3B). Inclusion of hirudin reduced fibrin(ogen) surface area coverage from $50 \pm 3$ $\%$ to $9 \pm 1 \%(p<0.05$; Fig. $3 C, D)$. Consequently, plasminogen binding decreased $83 \%$ ( $p$ $<0.05$; Fig. 3D), and overlap coefficients for plasminogen with platelets or fibrinogen were similar under these conditions ( $0.64 \pm 0.06$ and $0.72 \pm 0.07$, respectively) (Fig. 3B).

High-resolution confocal $z$ stacks of thrombi ${ }^{42}$ were performed to study the localisation of plasminogen during thrombus formation. An intense signal from platelet aggregates was evident at the base, consistent with deposition on the collagen/TF surface (Fig. 3E). Before visible fibrin fibre formation, plasminogen was detected around platelets in the base and centre $z$ stacks (Fig. $3 \mathrm{E}$ ). Following the onset of fibrin formation, fibrin(ogen) and plasminogen radiated from platelet aggregates at the base and extended over the platelet surface as shown by the centre and top $z$ stacks (Fig. 3E). During tPA-mediated fibrinolysis, maximal plasminogen signal was visualised at $t=3$ minutes, followed by a reduction in signal as the fibrin(ogen) was degraded (Fig. 3F). Despite a decrease in overall signal during fibrinolysis, the overlapcoefficient for plasminogen and fibrinogen remained high $(R>0.95)$. Prolonged perfusion of thrombi with buffer did not substantially alter the plasminogen signal, except in the presence of hirudin, illustrating the role of fibrin in stabilising the binding of plasminogen (not shown).

We further examined the pattern of fibrin degradation in thrombi during tPAmediated fibrinolysis. At $t=0$ minutes, the most intense fluorescent signal was directly associated with the platelet surface, indicative of dense fibrin in these areas (Fig. 4A, B). Subtraction analysis revealed that platelet-associated fibrin is extremely 

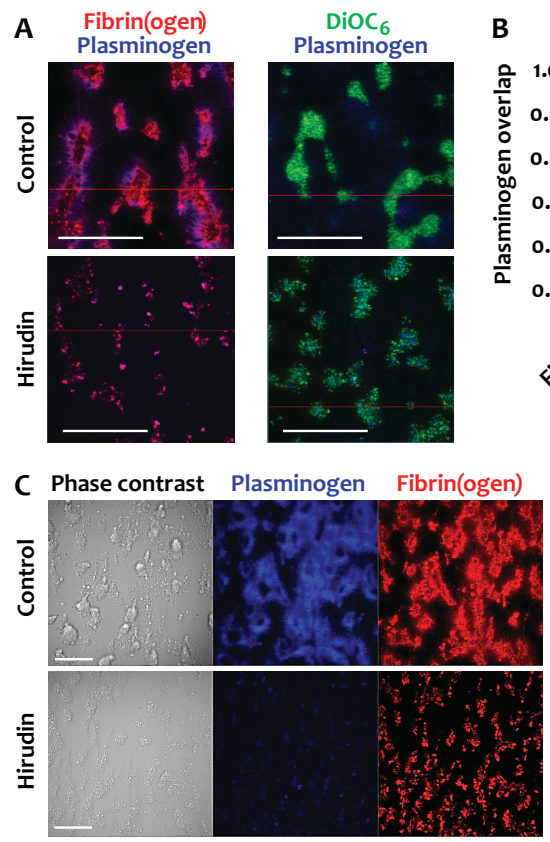

E
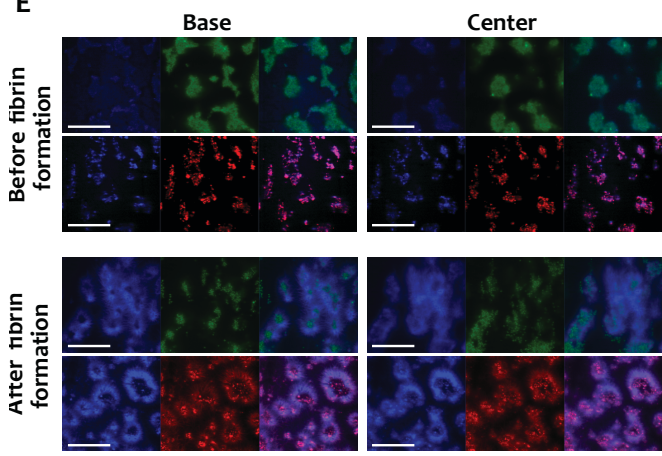

B
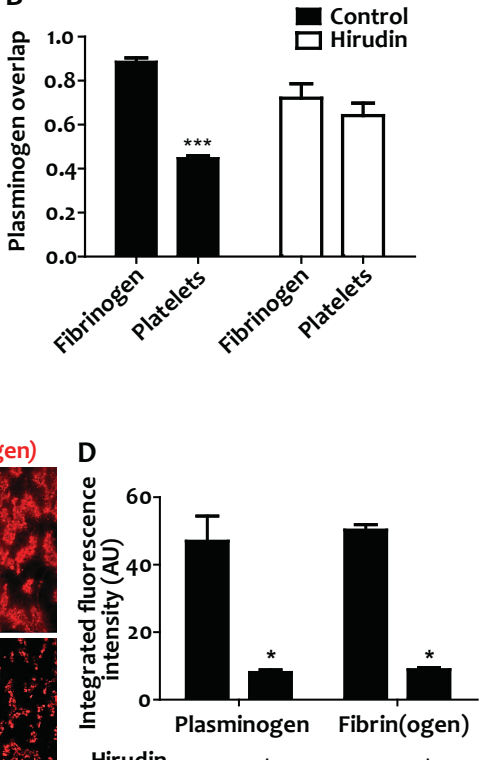

Top
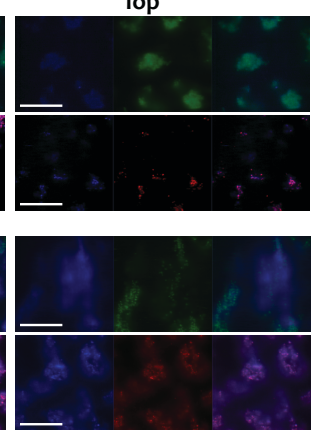

$\mathbf{F}$
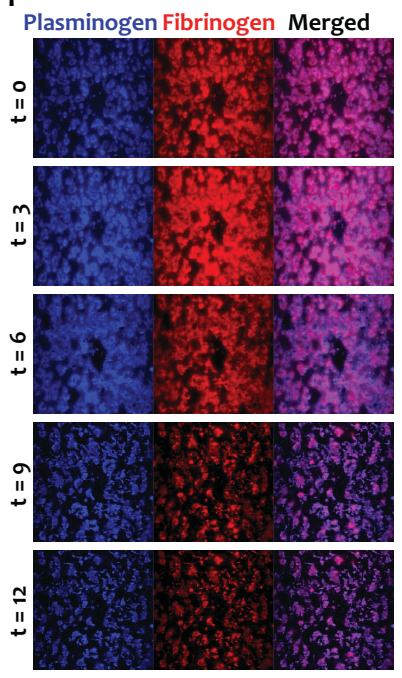

Figure 3. Plasminogen localisation within thrombi. Thrombi were formed by whole blood perfusion (1,000 $\left.\mathrm{s}^{-1}\right)$ over a collagen/TF-coated surface with or without hirudin $(3 \mu \mathrm{g} / \mathrm{mL})$. Platelets labelled with $\mathrm{DiOC}_{6}$ $(0.5 \mu \mathrm{g} / \mathrm{mL})$ or fibrinogen-OG488 $(75 \mu \mathrm{g} / \mathrm{mL})$ was included. Thrombi were perfused with plasminogenDL633 $(0.8 \mu \mathrm{M})$. (A) Representative overlays of plasminogen (blue) and fibrin(ogen) (red), or platelets (green). (B) Overlap coefficients (R) for plasminogen with fibrin(ogen) and/or platelets as determined using Zeiss Live 7 software. (C) Representative images of thrombi labelled for plasminogen-DL633 (blue) and fibrin(ogen)-OG488 (red). (D) Quantification of plasminogen and fibrinogen fluorescence intensity (AU). (E) Confocal $z$ stacks were recorded of labelled thrombi (16-bit images of 1024 x 1024 pixels; 106 x $106 \mu \mathrm{m}$; stack distance $0.5 \mu \mathrm{m}$; 50 slices). Representative images and overlays of plasminogen (blue) and platelets (green), or fibrin(ogen) (red) taken from $z$ stacks at the base $(0 \mu \mathrm{m})$, centre $(10 \mu \mathrm{m})$, and top $(20 \mu \mathrm{m}$ ) of thrombi before (top panel) or after (bottom panel) visible fibrin formation. (F) Thrombi were formed as above after preincubation with plasminogen-DL633 (0.8 $\mu \mathrm{M})$ and fibrinogen-OG488 (75 $\mu \mathrm{g} / \mathrm{mL}$ ) in the presence of $10 \mathrm{nM} \mathrm{tPA}$. The fluorescent thrombi were perfused $(\mathrm{t}=0$ ) with buffer 1 (Hepes buffer, pH 7.45) for 8 minutes, followed by buffer 2 (Hepes buffer, pH 7.45 with $10 \mathrm{nM}$ tPA) for up to 28 minutes. Shown are representative images of thrombi labelled for fibrin(ogen) (red) and plasminogen (blue)(bars, $50 \mu \mathrm{m}) .{ }^{*} p<0.05 ; * * * p<0.001$. Data represented as means \pm SEM, $n \geq 3$. 
A

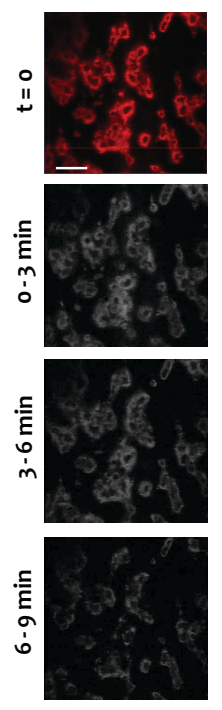

B
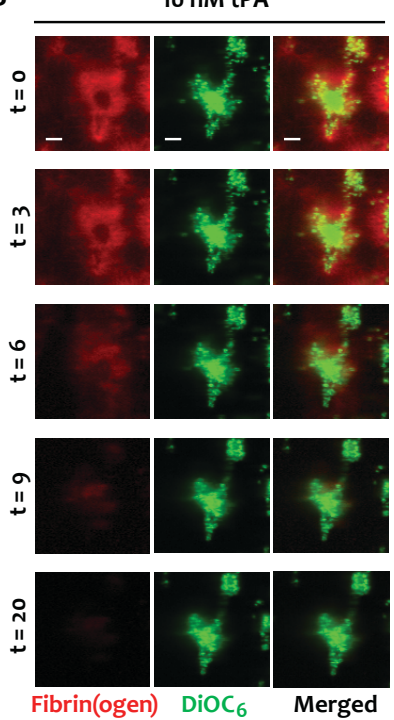
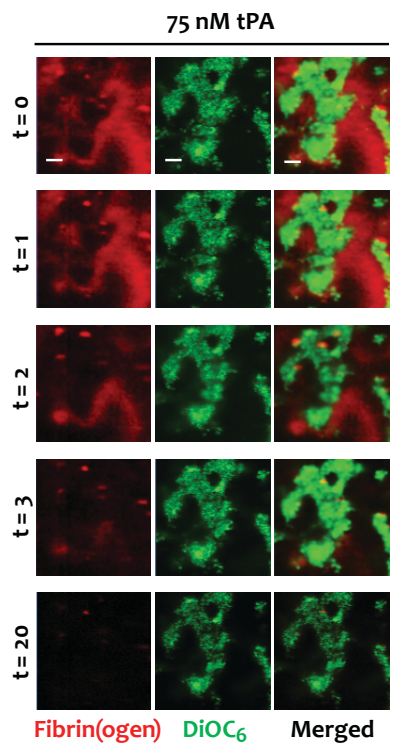

Figure 4. Fibrinolysis is delayed in fibrin immediately proximal to the platelet surface. Thrombi were formed by whole blood perfusion $\left(1,000 \mathrm{~s}^{-1}\right)$ over a collagen/TF-coated surface for 7 minutes in the presence of $10 \mathrm{nM}$ tPA. Thrombi were subsequently perfused $(t=0)$ with Hepes buffer, $\mathrm{pH} 7.45$ for 8 minutes, and then Hepes buffer containing $10 \mathrm{nM}$ tPA. (A) Representative image of fibrin(ogen)-AF647 staining on thrombi at the start and images subjected to subtraction analysis of fibrinogen fluorescence $(n=3)$. (B) Blood samples were preincubated with DiOC $_{6}(0.5 \mu \mathrm{g} / \mathrm{mL})$ to label platelets and fibrinogenAF647 $(16.7 \mu \mathrm{g} / \mathrm{mL})$. Thrombi were allowed to form as above in the presence of $10 \mathrm{nM}$ or $75 \mathrm{nM} \mathrm{tPA}$. The fluorescent thrombi were perfused $(t=0$ ) with buffer 1 (Hepes buffer, $\mathrm{pH} 7.45$ ) for 8 minutes, followed by buffer 2 (Hepes buffer, $\mathrm{pH} 7.45$ with indicated concentration tPA) until lysis was complete. Representative images and overlays of platelets (green) and fibrin(ogen) (red) during lysis with $10 \mathrm{nM}$ or $75 \mathrm{nM}$ tPA. Images are representative of $n=8$ (bars, $50 \mu \mathrm{m})$.

resistant to lysis (Fig. 4A). Higher magnification images of single thrombi revealed that the fibrin fibres distal to platelet aggregates were the first to be degraded (Fig. 4B) (10 nM tPA, o to 6 minutes), whereas fibrin adjacent to platelets was the last to lyse (consistent with Fig. 4A). A faster rate of lysis was observed with $75 \mathrm{nM}$ tPA, but the same pattern of degradation was visualised (Fig. 4B). These data indicate that tPA concentration affects the kinetics but not the pattern of fibrin degradation.

\section{Plasminogen activation is enhanced on the stimulated platelet membrane}

Plasmin activity was analysed on the surface of resting platelets, or platelets stimulated with CVX plus thrombin or TRAP-6. In the absence of exogenous PA, minimal plasmin activity was detected, except when platelets were stimulated with thrombin/CVX ( $p<0.001$; Fig. 5A). The addition of $1 \mathrm{nM}$ tPA or uPA increased plasmin generation on unstimulated and stimulated platelets (Fig. 5B, C). uPA-mediated plasmin activity was significantly augmented with thrombin/CVX and TRAP-6/CVX compared with unstimulated platelets. In contrast, tPA-mediated plasmin generation 
A

No PA

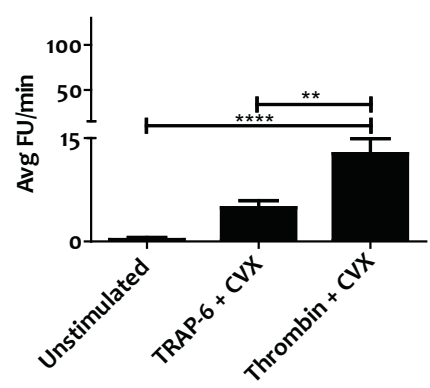

B

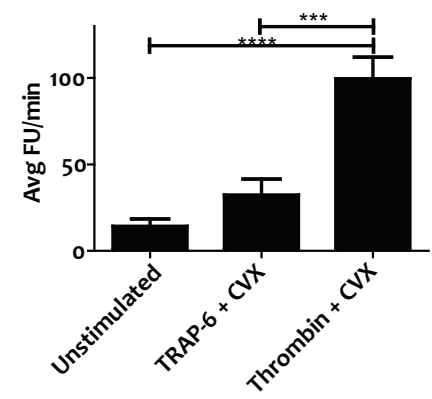

C UPA

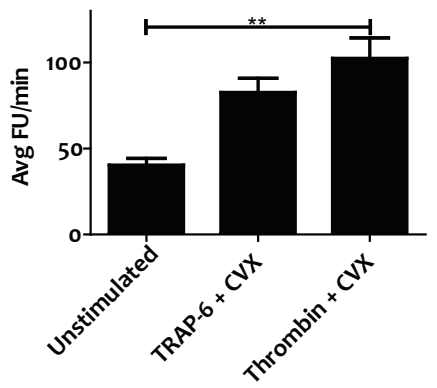

Figure 5. Platelet stimulation enhances plasminogen activation. (A) Washed platelets $\left(5 \times 10^{8}\right.$ platelets/ $\mathrm{mL})$ were stimulated with CVX $(100 \mathrm{ng} / \mathrm{mL})+$ thrombin $(100 \mathrm{nM})$ or TRAP-6 $(15 \mu \mathrm{M})$ for 45 minutes. (B-C) Platelets were then diluted to a final concentration of $6 \times 10^{7}$ platelets $/ \mathrm{mL} \pm \operatorname{tPA}$ or uPA $(1 \mathrm{nM})$ in the presence of hirudin $(0.3 \mu \mathrm{g} / \mathrm{mL})$ and D-Val-Leu-Lys-7-amido-4-methylcoumarin $(0.35 \mathrm{mM})$. Plasmin generation was measured as fluorescence release and quantified as average FU per minute. ${ }^{* *} p<0.01$; $* * * p<0.001 ; * * * * p<0.0001$ compared with unstimulated platelets. Data are expressed as means \pm SEM, $n=5$. FU, fluorescence unit.

was only significantly enhanced when platelets were stimulated with thrombin/ CVX. This difference may be explained by the fact that thrombin, unlike TRAP-6, cleaves fibrinogen indicating that platelet-associated fibrin supports tPA-mediated plasminogen activation.

\section{Plasminogen binds to PS-exposing platelets}

Flow cytometry was used to analyse direct binding of fibrinogen and plasminogen to washed stimulated platelets. Stimulation of platelets with TRAP-6/CVX or thrombin/ CVX significantly augmented binding of fibrinogen-AF647 $(p<0.01$ and $p<0.0001$, respectively) (Fig. $6 \mathrm{~A}$ ), with maximal binding observed with thrombin/CVX stimulation $(p<0.001)$. The percentage of unstimulated platelets that bound fibrinogen-AF647 was much lower $(5.6 \pm 3.5 \%)$ than the number that bound plasminogen-DL633 (29.5 $\pm 10.3 \%$ ). However, binding of plasminogen-DL633 was still significantly enhanced by stimulation (TRAP-6/CVX, 87.2 $\pm 2.7 \%, p<0.001$ and thrombin/CVX, $94.5 \pm 2.1 \%, p<$ 0.0001) (Fig. 6B). A comparable number of platelets bound plasminogen-DL633 when stimulated with thrombin/CVX or TRAP-6/CVX, however, the median fluorescence intensity (MFI) was nine-fold higher with thrombin/CVX stimulation. Thrombin stimulation alone generated similar numbers of plasminogen-positive platelets, consistent with previous reports, ${ }^{22}$ but MFI was reduced twofold compared with thrombin/CVX (data not shown).

Staining with annexin A5-FITC revealed that $66.2 \pm 12.2 \%$ of the thrombin/CVXstimulated platelets exposed PS. The majority of plasminogen-positive thrombin/ CVX- stimulated platelets were PS-exposing (65.8 $\pm 12.6 \%$; Fig. $6 \mathrm{~B})$, in contrast to unstimulated and TRAP-6/CVX-stimulated platelets $(3.8 \pm 2.4 \%$ and $12.6 \pm 1.0 \%$, 


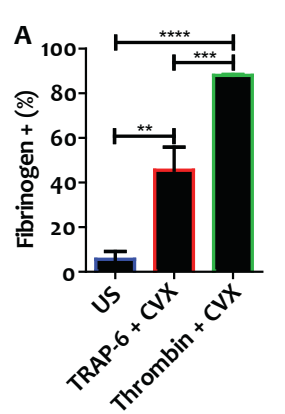

C

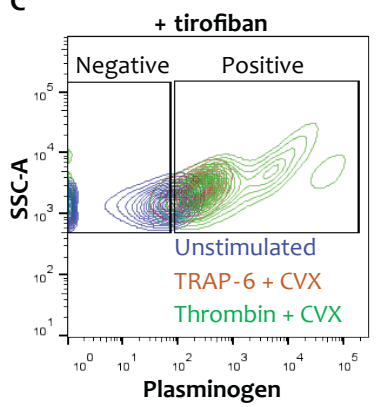

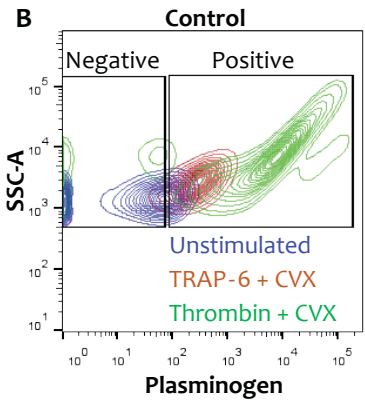

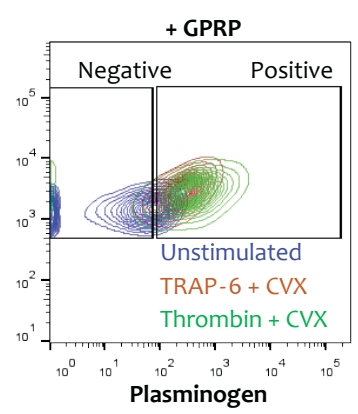

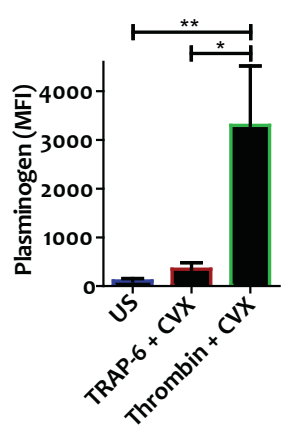
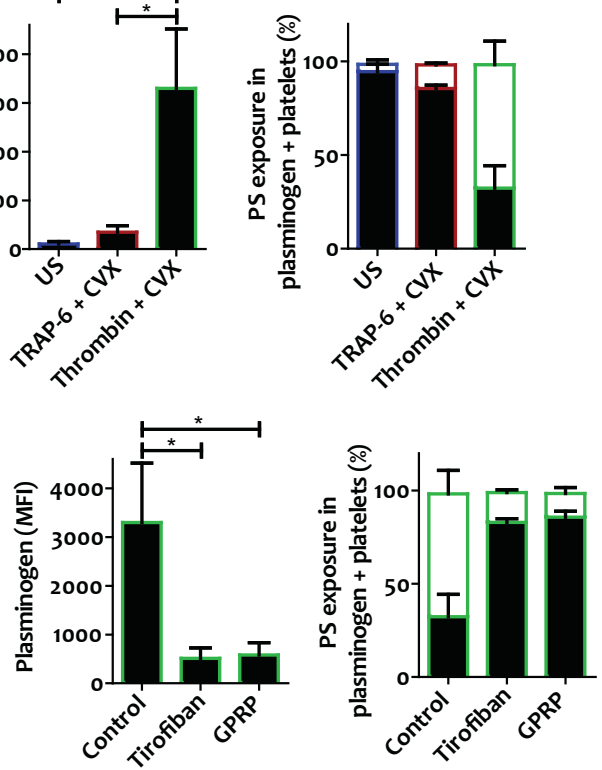

Figure 6. Activation of platelets stimulates binding of plasminogen. Platelets $\left(2 \times 10^{8} / \mathrm{mL}\right)$ were stimulated with thrombin $(100 \mathrm{nM})+\mathrm{CVX}(100 \mathrm{ng} / \mathrm{mL})$ or TRAP-6 $(15 \mu \mathrm{M})+\mathrm{CVX}$. After 40 minutes stimulation, either (A) fibrinogen-AF647 $(16.7 \mu \mathrm{g} / \mathrm{mL})$ or (B) plasminogen-DL633 $(0.27 \mathrm{mM})$ was added for 5 minutes before the addition of annexin A5-FITC (1:20 dilution), followed by Hepes buffer ( $\mathrm{pH} 7.45$ ) containing $2 \mathrm{mM} \mathrm{CaCl}_{2}$. Platelets were then analysed by flow cytometry. (A) Percentage of fibrinogen-AF647-positive platelets. (B) Representative contour plots showing side scatter (SSC-A) against plasminogen-DL633, gated on unstained platelets (left). Plasminogen-DL633 binding quantified as MFI (middle) and the percentage of plasminogen-positive platelets that are PS-exposing (open bars) or negative (closed bars) (as indicated by annexin A5-FITC) are shown (right). (C) Representative contour plots of thrombin/CVX-stimulated platelets in the presence of GPRP $(5 \mathrm{mM})$ or tirofiban $(1 \mu \mathrm{g} / \mathrm{mL})$ (left). Plasminogen-DL633 binding quantified as MFI (middle) and the percentage of plasminogen-positive platelets that are PS-exposing (open bars) or negative (closed bars) are shown (right). Expressed as mean \pm SEM. ${ }^{*} p<0.05 ;{ }^{* *} p<0.01$; $* * * p<0.001 ; * * * * p<0.0001 ; n=3$.

respectively). These differences reinforce a role for fibrin in plasminogen binding. Consistent with this inclusion of tirofiban, a potent inhibitor of the $\alpha_{11 b} \beta_{3}$, decreased the MFI of plasminogen-DL633 on thrombin/CVX-stimulated platelets sevenfold $(p<0.05)$ (Fig. 6C), but did not change the number of positive platelets. GPRP is analogous to the amino-terminus of $A \alpha$-chain and $B \beta$-chain of fibrinogen and inhibits fibrin polymerisation. GPRP reduced the MFI of plasminogen-DL633 in thrombin/CVXstimulated platelets sixfold ( $p<0.05$ ) (Fig. 6C), whereas Gly-Pro-Pro-Pro, a negative control peptide, had no effect (not shown). The change in relative amount of plasminogen bound in the presence of tirofiban or GPRP correlated with the number of PS-exposing platelets. No significant additional reduction in plasminogen binding to thrombin/CVX-stimulated platelets occurred with both tirofiban and GPRP. Neither tirofiban nor GPRP altered plasminogen binding in unstimulated or TRAP-6 + CVX- 
A

B

Control

+ Tirofiban

+ GPRP

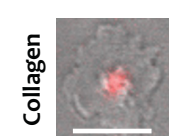

Annexin A5 Plasminogen Merged

Annexin A5 Plasminogen Merged

Annexin A5 Plasminogen Merged
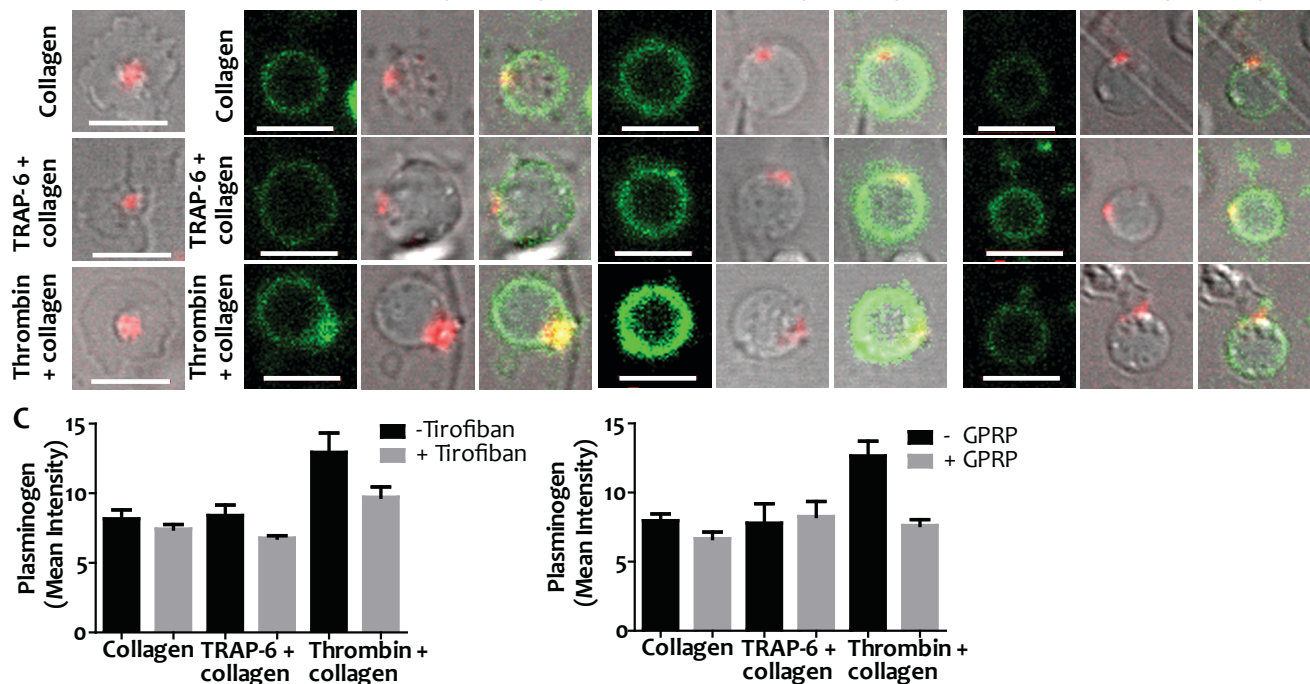

D

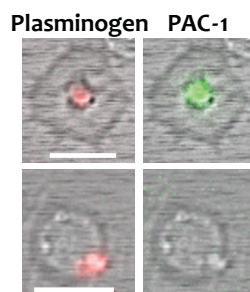

E
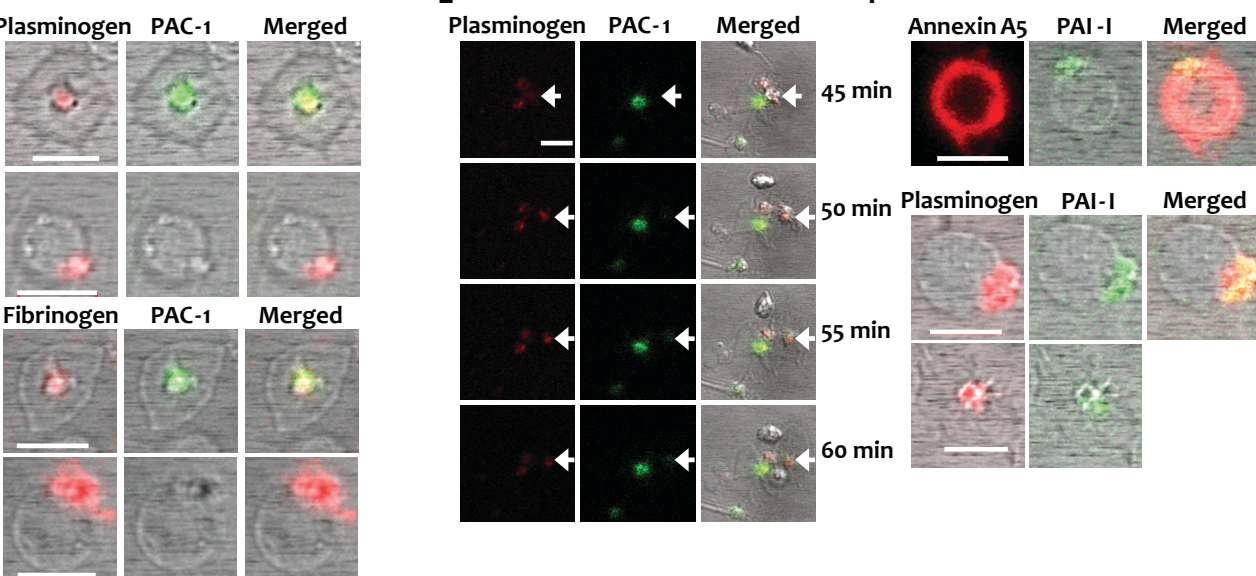

Plasminogen PAI-I Merged

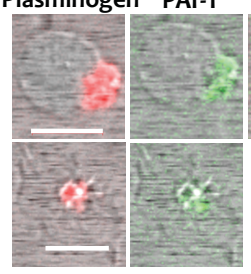

Figure 7. Plasminogen localises in caps of PS-exposing platelets. Platelets $\left(0.5 \times 10^{8} / \mathrm{mL}\right)$ were adhered to a collagen $(0.6 \mu \mathrm{g}) \pm$ thrombin $(3 \mathrm{pmol})$ or \pm TRAP-6 $(0.45 \mu \mathrm{mol})$ coated slide \pm GPRP $(5 \mathrm{mM})$ or \pm tirofiban $(1 \mu \mathrm{g} / \mathrm{mL})$. After 40 minutes incubation, plasminogen-DL633 (0.8 $\mu \mathrm{M})$ or fibrinogen-AF647 (16.7 $\mu \mathrm{g} / \mathrm{mL})$ was added for 5 minutes. (A) Spread PS-negative platelets showing plasminogen-DL633 binding. (B) Plasminogen-DL633 binding on PS-positive platelets (left) \pm tirofiban (middle), or GPRP (right). Annexin A5-FITC (1:20 dilution) and $2 \mathrm{mM} \mathrm{CaCl}_{2}$ were added immediately prior to imaging. (C) Representative quantification of plasminogen-DL633 binding expressed as mean intensity \pm SEM determined using Bitplane's Imaris x64 software. (D) Platelets on collagen + thrombin surface stained with PAC-1-FITC showing plasminogen-DL633 (top panel) and fibrinogen-AF647 (bottom panel) binding on spread and balloon-shaped subpopulations. (E) Platelets on collagen + thrombin surface stained with PAC-1-FITC and showing plasminogen-DL633 binding. Arrows indicate a platelet with plasminogen bound that tethers to a spread plasminogen-positive platelet, which subsequently expresses active $\alpha_{11 b} \beta_{3}$, represented by positive PAC-1 staining. Images were recorded every minute for 60 minutes and selected images are shown. (F) Staining for platelet-derived PAI-1 in collagen + thrombin-stimulated platelets using an antiPAI-1-DL488 antibody and PS (annexin A5-AF647) (top). PAl-1 co-localisation with plasminogen-DL633 in balloon-shaped (middle) and spread platelets (bottom)(bars, $5 \mu \mathrm{m}$ ). Images are representative of $n=3$. 
stimulated platelets (not shown). These results imply that $\alpha_{1 \mathrm{lb}} \beta_{3}$ activation and fibrin polymerisation are essential for maximal binding of plasminogen to PS-exposing platelets.

\section{Plasminogen localises in caps on PS-exposing platelets}

Plasminogen binding to the surface of live unpermeablised platelets was visualised by fluorescent confocal microscopy. Slides coated with collagen alone or in combination with TRAP- 6 or thrombin generated 2 distinct platelet subpopulations. PS-negative platelets with a spread morphology accumulated plasminogen-DL633 in the centre over the granulomere, whereas balloon-shaped PS-exposing platelets (indicated by annexin A5-FITC binding) bound plasminogen in a protruding 'cap' (Fig. 7A, B). Increased plasminogen-DL633 binding to the cap was observed with thrombin stimulation. Tirofiban and GPRP diminished, but did not abolish, plasminogen-DL633 binding on PS-exposing platelets (Fig. 7B, C), consistent with flow cytometry data. These data indicate that fibrin augments the localisation of plasminogen in caps of PS-exposing platelets. Exogenous fibrinogen-AF647 also bound to the cap of balloonshaped platelets (Fig. 7D). The presence of active $\alpha_{11 b} \beta_{3}$ was analysed using an antibody to PAC-1. PAC-1 co-localised with plasminogen and fibrinogen in the centre of spread platelets but was absent from the caps of balloon-shaped PS-exposing platelets (Fig. 7D). A time course following platelet stimulation revealed that plasminogen-DL633 was present in spread platelets prior to visualisation of $\mathrm{PAC}-1$, indicating that binding of plasminogen to this subpopulation is not dependent on active $\alpha_{11 b} \beta_{3}$ (Fig. 7E). PAI1 is contained in platelet $\alpha$-granules and is released upon stimulation. ${ }^{15}$ We found that PAI-1 co-localised with plasminogen in both the protruding cap of PS-exposing platelets and in spread platelets (Fig. 7F). These data suggest that these caps serve as a focal point for localisation of pro- and anti-fibrinolytic proteins.

\section{Discussion}

Knowledge of the location of plasminogen in thrombi is crucial to our understanding of initiation, regulation, and progression of fibrinolysis under physiological shear rates. In this study, we used a novel whole blood flow model and found that plasminogen largely associated with the fibrin network emanating from platelet aggregates. A smaller but significant pool directly associated with the platelet surface. We found that subpopulations of platelets showed different distributions of plasminogen, with it localised to distinct caps on PS-exposing platelets.

The composition of the fibrin network affects the rate of fibrinolysis, with areas of dense thin fibres being lysed more slowly. ${ }^{44}$ Fibrin also acts as a cofactor for tPAmediated plasminogen activation, with thin fibres being less effective than thick fibres. ${ }^{44}, 45$ We found that fibrinolysis was accelerated by flow, irrespective of the shear rate applied, and was dependent on the PA and its concentration. Fibrinolysis 
with tPA was more efficient, consistent with its binding to fibrin, which also protects it from inhibition by PAl-1. ${ }^{46}$ Partial degradation of fibrin by plasmin exposes new carboxyl-terminal lysine residues, which enhance binding of plasminogen thereby accelerating lysis. ${ }^{47-52}$ In our flow model, we visualised this phenomenon in real time as a wave of plasminogen accumulation following initiation of tPA-mediated fibrinolysis, which subsequently receded as lysis progressed. Cardiothoracic patients examined here received the lysine analogue transexamic acid to prevent on-going fibrinolysis during surgery. Prior to drug administration, lysis of thrombi from these patients was similar to controls, however, following treatment, no lysis was detected during the time frame. The use of transexamic acid in this patient group exemplifies the importance of plasminogen binding to exposed lysine binding sites on partially degraded fibrin. Together, these results highlight the role of the fibrin network in orchestrating its own destruction by localising the fibrinolytic process to its surface. We found that lysis of fibrin proximal to platelets was markedly delayed, in line with previous observations in static clots..$^{53}$ The fibrin network around platelets is composed of thin densely packed fibres, ${ }^{44,53}$ which are less efficient at supporting plasminogen activation and may represent a protective mechanism to stabilise early thrombi and possibly prevent against embolization.

We showed that the majority of thrombin/CVX-stimulated platelets that bound plasminogen were PS-exposing and localised plasminogen to a distinct cap on their surface. Coated platelets accumulate fibrin(ogen) on their surface ${ }^{9,10}$ and Abaeva et al..$^{54}$ recently reported that platelet-derived fibrin(ogen) and thrombospondin are focused in a cap on the activated membrane. Our results indicate that exogenous fibrinogen also binds the cap on PS-exposing platelets co-localising with plasminogen. Interestingly, we have recently shown that platelet-derived factor XIII-A is found in the cap of PS-exposing platelets..$^{55}$ The presence of activated FXIII-A potentially stabilises fibrin emanating from the platelet surface mechanically, via cross-linking of fibrin, and protects against fibrinolytic degradation by cross-linking $a_{2} A P .{ }^{55}$ Coated platelets express $\alpha_{11 b} \beta_{3}$ on their surface that becomes inactivated after occupation by a ligand such as fibrinogen. ${ }^{4,10,56}$ PS-exposing platelets in this study did not express the active $\alpha_{11 \mathrm{~b}} \beta_{3}$, as demonstrated by the lack of PAC-1 staining in the cap. Intriguingly, despite the lack of active integrin, we show that blocking $\alpha_{11 b} \beta_{3}$ and fibrin polymerisation attenuates plasminogen binding to PS-exposing platelets. Consistent with this, platelets from patients with Glanzmann's thrombasthenia who lack $\alpha_{11 b} \beta_{3}$ display reduced plasminogen binding. ${ }^{57}$ Inhibition of $\alpha_{11 b} \beta_{3}$ initiates thrombin signalling through GPIb, which requires polymerising fibrin. ${ }^{58}$ It is unlikely that GPIb has a role in plasminogen binding, as levels on resting and thrombin-stimulated platelets from patients with Bernard-Soulier syndrome, who lack GPIb, are similar to controls. ${ }^{57}$ We observed a significant reduction in plasminogen MFI when platelets were treated with GPRP to inhibit fibrin polymerisation. Together, these data indicate that $\alpha_{11 b} \beta_{3}$ has an indirect role in plasminogen binding via fibrinogen, which is subsequently polymerised to fibrin, thereby amplifying the number of plasminogen binding sites. 
Platelets contain both $\alpha_{2} \mathrm{AP}^{59}$ and PAI-1 ${ }^{15,60-63}$ that are released upon stimulation. We found that a proportion of platelet PAl-1 co-localised with plasminogen binding in PS-exposing and spread platelets. Therefore, local plasminogen activation on the platelet surface must overcome PAI-1 inhibition to generate functional plasmin. Plasmin activity was detected in effluents collected during fibrinolysis with $75 \mathrm{nM}$ tPA, but not with $10 \mathrm{nM}$ tPA, despite this concentration achieving lysis of thrombi. The lack of quantifiable plasmin in effluents can be explained by protection from $a_{2}$ AP inhibition via binding to the fibrin surface ${ }^{64}$ and the consumption of free enzyme by inhibitors. In addition to enhanced plasminogen binding to thrombin/CVX-stimulated platelets, we also showed an increase in plasmin activity, even in the absence of exogenous PAs, suggesting the presence of endogenous PA activity on platelets. Higher plasmin activity was observed in thrombin/CVX-stimulated platelets compared with TRAP-6/ CVX stimulation with tPA, which can be attributed to the presence of fibrin. ${ }^{65}$ Plasmin treatment of platelets augments the number of binding sites for plasminogen in both unstimulated and ADP-stimulated platelets, ${ }^{24,25}$ and enhances tPA activity. In contrast, plasmin generation on platelets treated with uPA was significantly augmented by TRAP-6/CVX and thrombin/CVX stimulation. Cell-bound UPA is capable of activating lys-plasminogen and $\varepsilon A C A$-liganded plasminogen, which has an open conformation similar to lys-plasminogen, without the requirement of being bound to the same cell surface. ${ }^{23}$ Indeed, a crosstalk mechanism of activation of platelet-bound plasminogen by uPA bound to monocytes or endothelial microparticles has been described, ${ }^{23}$ and our work has previously shown that scuPA-mediated fibrinolysis was enhanced by platelet-associated plasminogen. ${ }^{19}$ These reports highlight the different mechanisms by which these PAs activate plasminogen and regulate fibrinolysis.

Recently, a hierarchical thrombus structure has been observed in vivo, in which 2 discrete regions of platelet activation were observed. ${ }^{2}$ The inner core is rich in fibrin and thrombin and consists of tightly packed platelets with extensive a-granule release. A loosely packed shell of platelets with minimal a-granule release encases the core. Here, we demonstrate plasminogen accumulation around platelet aggregates at the core of the thrombus. As fibrin forms, plasminogen co-localising with fibrin(ogen) radiates from the aggregates extending over their surface into the loosely packed shell. We found a high overlap coefficient for plasminogen and fibrin(ogen) with the most intense fibrin(ogen) signal in direct association with platelets. Thrombi perfused with whole blood containing heparin lysed significantly faster than whole blood alone, highlighting the importance of on-going fibrin formation in these thrombi as a result of procoagulant activity. Notable work by Stalker et al. ${ }^{66}$ has recently shown that integrin $\alpha_{1 \mathrm{lb}} \beta_{3}$ outside-in signalling localises thrombin activity within the core region of thrombi by minimising solute transport. Our studies on plasminogen localisation and plasmin activity suggest that it may be regulated by similar mechanisms via the interaction of fibrin(ogen) with $\alpha_{1 \mathrm{ll}} \beta_{3}$ within the microenvironment of the thrombus.

In conclusion, a functional pool of plasminogen accumulates on the platelet membrane following strong agonist stimulation. Under physiological flow 
conditions, plasminogen binds directly to platelets but is predominantly visualised on fibrin(ogen) radiating from PS-exposing platelets, and it is this pool that fluctuates during fibrinolysis. Two subpopulations of platelets were observed with distinct plasminogen and fibrinogen binding characteristics. Spread platelets accumulate plasminogen and fibrinogen centrally over the granulomere via a $\alpha_{11 b} \beta_{3}$-dependent manner. In contrast, PS-exposing platelets, such as those found in the core of a thrombus bind plasminogen to protruding caps via platelet-derived fibrin(ogen) and display an enhanced capacity to generate plasmin on their surface. These data suggest a role for PS-exposing platelets in modulating local fibrinolysis under physiological flow conditions within the microenvironment of the thrombus. Interestingly, a recent report described thrombolysis with scuPA fused to single-chain antibody fragments, which binds activated $\alpha_{11 b} \beta_{3}$, in a plasminogen-dependent manner. ${ }^{67}$ Targeting this sub-population of platelets in thrombi could prove useful in the development of novel thrombolytics to augment local fibrinolysis.

\section{Authorship contribution}

C.S. Whyte and F. Swieringa performed the research, analysed the data, and wrote the manuscript; T.G. Mastenbroek and A.S. Liokiene performed the research; M.D. Lancé contributed vital patient samples; P.E.J.van der Meijden supervised the research; and J.W.M. Heemskerk and N.J. Mutch supervised the research, analysed the data, and wrote the manuscript.

\section{Disclosure of conflict of interest}

The authors declare no competing financial interests.

\section{Acknowledgments}

The authors thank the Microscopy and Histology Core Facility and the lain Fraser Cytometry Centre at the University of Aberdeen for excellent advice and use of the facilities. This work was supported by grants from the British Heart Foundation (FS/11/2/28579) (N.J.M., A.S.L.) and (PG/11/1/28461) (N.J.M., C.S.W.), the National Health Service Grampian Endowment (grant 14/43) (C.S.W., N.J.M.), Friends of Anchor (N.J.M.), the Landsteiner Foundation for Blood Transfusion Research (1006) (F.S., P.E.J.v.d.M., J.W.M.H.), and the Cardiovascular Centre Maastricht (F.S., P.E.J.v.d.M., J.W.M.H.). Travel for this project was supported by a grant from the British Society for Haemostasis and Thrombosis (N.J.M., C.S.W.).

\section{References}

1. Heemskerk JW, Mattheij NJ, Cosemans JM. Platelet-based coagulation: different populations, different functions. J Thromb Haemost. 2013;11:2-16. 
2. Stalker TJ, Traxler EA, Wu J, Wannemacher KM, Cermignano SL, Voronov R, Diamond SL, Brass LF. Hierarchical organization in the hemostatic response and its relationship to the platelet-signaling network. Blood. 2013;121:1875-1885.

3. Berny MA, Munnix IC, Auger JM, Schols SE, Cosemans JM, Panizzi P, Bock PE, Watson SP, McCarty OJ, Heemskerk JW. Spatial distribution of factor Xa, thrombin, and fibrin(ogen) on thrombi at venous shear. PLoS One. 2010;5:e10415.

4. Munnix IC, Kuijpers MJ, Auger J, Thomassen CM, Panizzi P, Van Zandvoort MA, Rosing J, Bock PE, Watson SP, Heemskerk JW. Segregation of platelet aggregatory and procoagulant microdomains in thrombus formation: regulation by transient integrin activation. Arterioscler Thromb Vasc Biol. 2007;27:2484-2490.

5. Bevers EM, Comfurius P, Zwaal RF. Platelet procoagulant activity: physiological significance and mechanisms of exposure. Blood Rev. 1991;5:146-154.

6. Heemskerk JW, Bevers EM, Lindhout T. Platelet activation and blood coagulation. Thromb Haemost. 2002;88:186-193.

7. Cosemans JM, Schols SE, Stefanini L, De Witt S, Feijge MA, Hamulyák K, Deckmyn H, Bergmeier W, Heemskerk JW. Key role of glycoprotein Ib/V/IX and von Willebrand factor in platelet activationdependent fibrin formation at low shear flow. Blood. 2011;117:651-660.

8. Alberio L, Safa O, Clemetson KJ, Esmon CT, Dale GL. Surface expression and functional characterization of $\alpha$-granule factor $V$ in human platelets: effects of ionophore A23187, thrombin, collagen, and convulxin. Blood. 2000;95:1694-1702.

9. Dale GL. Coated-platelets: an emerging component of the procoagulant response. J Thromb Haemost. 2005;3:2185-2192.

10. Dale GL, Friese P, Batar P, Hamilton SF, Reed GL, Jackson KW, Clemetson KJ, Alberio L. Stimulated platelets use serotonin to enhance their retention of procoagulant proteins on the cell surface. Nature. 2002;415:175-179.

11. Szasz R, Dale GL. Thrombospondin and fibrinogen bind serotonin-derivatized proteins on COATplatelets. Blood. 2002;100:2827-2831.

12. Fay WP, Eitzman DT, Shapiro AD, Madison EL, Ginsburg D. Platelets inhibit fibrinolysis in vitro by both plasminogen activator inhibitor-1-dependent and -independent mechanisms. Blood. 1994;83:351-356.

13. Robbie LA, Booth NA, Croll AM, Bennett B. The roles of $\alpha_{2}$-antiplasmin and plasminogen activator inhibitor 1 (PAl-1) in the inhibition of clot lysis. Thromb Haemost. 1993;70:301-306.

14. Levi M, Biemond BJ, Van Zonneveld AJ, Ten Cate JW, Pannekoek H. Inhibition of plasminogen activator inhibitor-1 activity results in promotion of endogenous thrombolysis and inhibition of thrombus extension in models of experimental thrombosis. Circulation. 1992;85:305-312.

15. Booth NA, Simpson AJ, Croll A, Bennett B, MacGregor IR. Plasminogen activator inhibitor (PAI-1) in plasma and platelets. Br J Haematol. 1988;70:327-333.

16. Maynard DM, Heijnen HF, Horne MK, White JG, Gahl WA. Proteomic analysis of platelet a-granules using mass spectrometry. J Thromb Haemost. 2007;5:1945-1955.

17. Veljkovic DK, Rivard GE, Diamandis M, Blavignac J, Cramer-Borde EM, Hayward CP. Increased expression of urokinase plasminogen activator in Quebec platelet disorder is linked to megakaryocyte differentiation. Blood. 2009;113:1535-1542.

18. Coppinger JA, Cagney G, Toomey S, Kislinger T, Belton O, McRedmond JP, Cahill DJ, Emili A, Fitzgerald DJ, Maguire PB. Characterization of the proteins released from activated platelets leads to localization of novel platelet proteins in human atherosclerotic lesions. Blood. 2004;103:20962104.

19. Baeten KM, Richard MC, Kanse SM, Mutch NJ, Degen JL, Booth NA. Activation of single-chain urokinase-type plasminogen activator by platelet-associated plasminogen: a mechanism for stimulation of fibrinolysis by platelets. J Thromb Haemost. 2010;8:1313-1322. 
20. Plow EF, Doeuvre L, Das R. So many plasminogen receptors: why? J Biomed Biotechnol. 2012;2012:141806.

21. Adelman B, Michelson AD, Loscalzo J, Greenberg J, Handin RI. Plasmin effect on platelet glycoprotein Ib-von Willebrand factor interactions. Blood. 1985;65:32-40.

22. Miles LA, Plow EF. Binding and activation of plasminogen on the platelet surface. J Biol Chem. 1985;260:4303-4311.

23. Dejouvencel T, Doeuvre L, Lacroix R, Plawinski L, Dignat-George F, Lijnen HR, Angles-Cano E. Fibrinolytic cross-talk: a new mechanism for plasmin formation. Blood. 2010;115:2048-2056.

24. Loscalzo J, Pasche B, Ouimet H, Freedman JE. Platelets and plasminogen activation. Thromb Haemost. 1995;74:291-293.

25. Ouimet H, Freedman JE, Loscalzo J. Kinetics and mechanism of platelet-surface plasminogen activation by tissue-type plasminogen activator. Biochemistry. 1994;33:2970-2976.

26. Stricker RB, Wong D, Shiu DT, Reyes PT, Shuman MA. Activation of plasminogen by tissue plasminogen activator on normal and thrombasthenic platelets: effects on surface proteins and platelet aggregation. Blood. 1986;68:275-280.

27. Hall SW, Humphries JE, Gonias SL. Inhibition of cell surface receptor-bound plasmin by $a_{2}-$ antiplasmin and $\alpha_{2}$-macroglobulin. J Biol Chem. 1991;266:12329-12336.

28. Plow EF, Freaney DE, Plescia J, Miles LA. The plasminogen system and cell surfaces: evidence for plasminogen and urokinase receptors on the same cell type. J Cell Biol. 1986;103:2411-2420.

29. Robbie LA, Young SP, Bennett B, Booth NA. Thrombi formed in a Chandler loop mimic human arterial thrombi in structure and PAI-1 content and distribution. Thromb Haemost. 1997;77:510-515.

30. Varju I, Sotonyi P, Machovich R, Szabo L, Tenekedjiev K, Silva MM, Longstaff C, Kolev K. Hindered dissolution of fibrin formed under mechanical stress. J Thromb Haemost. 2011;9:979-986.

31. Falati S, Gross P, Merrill-Skoloff G, Furie BC, Furie B. Real-time in vivo imaging of platelets, tissue factor and fibrin during arterial thrombus formation in the mouse. Nat Med. 2002;8:1175-1181.

32. Magwenzi SG, Ajjan RA, Standeven KF, Parapia LA, Naseem KM. Factor XIII supports platelet activation and enhances thrombus formation by matrix proteins under flow conditions. $J$ Thromb Haemost. 2011;9:820-833.

33. Mutch NJ, Moore NR, Wang E, Booth NA. Thrombus lysis by UPA, scuPA and tPA is regulated by plasma TAFI. J Thromb Haemost. 2003;1:2000-2007.

34. Neeves KB, Maloney SF, Fong KP, Schmaier AA, Kahn ML, Brass LF, Diamond SL. Microfluidic focal thrombosis model for measuring murine platelet deposition and stability: PAR4 signaling enhances shear-resistance of platelet aggregates. J Thromb Haemost. 2008;6:2193-2201.

35. Van der Meijden PE, Schoenwaelder SM, Feijge MA, Cosemans JM, Munnix IC, Wetzker R, Heller $\mathrm{R}$, Jackson SP, Heemskerk JW. Dual $\mathrm{P}_{2} \mathrm{Y}_{12}$ receptor signaling in thrombin-stimulated plateletsinvolvement of phosphoinositide 3 -kinase $\beta$ but not $\gamma$ isoform in $\mathrm{Ca}^{2+}$ mobilization and procoagulant activity. FEBS J. 2008;275:371-385.

36. Van Kruchten R, Cosemans JM, Heemskerk JW. Measurement of whole blood thrombus formation using parallel-plate flow chambers - a practical guide. Platelets. 2012;23:229-242.

37. Lancé MD, Ninivaggi M, Schols SE, Feijge MA, Oehrl SK, Kuiper GJ, Nikiforou M, Marcus MA, Hamulyák K, Van Pampus EC, Ten Cate H, Heemskerk JW. Perioperative dilutional coagulopathy treated with fresh frozen plasma and fibrinogen concentrate: a prospective randomized intervention trial. Vox Sang. 2012;103:25-34.

38. Munnix IC, Strehl A, Kuijpers MJ, Auger JM, Van der Meijden PE, Van Zandvoort MA, Oude Egbrink MG, Nieswandt B, Heemskerk JW. The glycoprotein VI-phospholipase C $\gamma_{2}$ signaling pathway controls thrombus formation induced by collagen and tissue factor in vitro and in vivo. Arterioscler Thromb Vasc Biol. 2005;25:2673-2678.

39. Siljander P, Farndale RW, Feijge MA, Comfurius P, Kos S, Bevers EM, Heemskerk JW. Platelet adhesion enhances the glycoprotein $\mathrm{VI}$-dependent procoagulant response: Involvement of p38 
MAP kinase and calpain. Arterioscler Thromb Vasc Biol. 2001;21:618-627.

40. Heemskerk JW, Feijge MA, Henneman L, Rosing J, Hemker HC. The $\mathrm{Ca}^{2+}$-mobilizing potency of a-thrombin and thrombin-receptor-activating peptide on human platelets: concentration and time effects of thrombin-induced $\mathrm{Ca}^{2+}$ signaling. Eur J Biochem. 1997;249:547-555.

41. Booth NA, MacGregor IR, Hunter NR, Bennett B. Plasminogen activator inhibitor from human endothelial cells. Purification and partial characterization. Eur J Biochem. 1987;165:595-600.

42. De Witt SM, Swieringa F, Cavill R, Lamers MM, Van Kruchten R, Mastenbroek T, Baaten C, Coort S, Pugh N, Schulz A, Scharrer I, Jurk K, Zieger B, Clemetson KJ, Farndale RW, Heemskerk JW, Cosemans JM. Identification of platelet function defects by multi-parameter assessment of thrombus formation. Nat Commun. 2014;5:4257.

43. Gallino A, Haeberli A, Hess T, Mombelli G, Straub PW. Fibrin formation and platelet aggregation in patients with acute myocardial infarction: effects of intravenous and subcutaneous low-dose heparin. Am Heart J. 1986;112:285-290.

44. Collet JP, Park D, Lesty C, Soria J, Soria C, Montalescot G, Weisel JW. Influence of fibrin network conformation and fibrin fiber diameter on fibrinolysis speed: dynamic and structural approaches by confocal microscopy. Arterioscler Thromb Vasc Biol. 2000;20:1354-1361.

45. Gabriel DA, Muga K, Boothroyd EM. The effect of fibrin structure on fibrinolysis. J Biol Chem. 1992;267:24259-24263.

46. Thelwell C, Longstaff C. The regulation by fibrinogen and fibrin of tissue plasminogen activator kinetics and inhibition by plasminogen activator inhibitor 1. J Thromb Haemost. 2007;5:804-811.

47. Bok RA, Mangel WF. Quantitative characterization of the binding of plasminogen to intact fibrin clots, lysine-sepharose, and fibrin cleaved by plasmin. Biochemistry. 1985;24:3279-3286.

48. Harpel PC, Chang TS, Verderber E. Tissue plasminogen activator and urokinase mediate the binding of Glu-plasminogen to plasma fibrin I. Evidence for new binding sites in plasmin-degraded fibrin I. J Biol Chem. 1985;260:4432-4440.

49. Suenson E, Lutzen O, Thorsen S. Initial plasmin-degradation of fibrin as the basis of a positive feedback mechanism in fibrinolysis. Eur J Biochem. 1984;140:513-522.

50. Tran-Thang C, Kruithof EK, Atkinson J, Bachmann F. High-affinity binding sites for human Gluplasminogen unveiled by limited plasmic degradation of human fibrin. Eur J Biochem. 1986;160:599604.

51. Tran-Thang C, Kruithof EK, Bachmann F. Tissue-type plasminogen activator increases the binding of glu-plasminogen to clots. J Clin Invest. 1984;74:2009-2016.

52. Fleury V, Angles-Cano E. Characterization of the binding of plasminogen to fibrin surfaces: the role of carboxy-terminal lysines. Biochemistry. 1991;30:7630-7638.

53. Collet JP, Montalescot G, Lesty C, Weisel JW. A structural and dynamic investigation of the facilitating effect of glycoprotein Ilb/IIla inhibitors in dissolving platelet-rich clots. Circ Res. 2002;90:428-434.

54. Abaeva AA, Canault M, Kotova YN, Obydennyy SI, Yakimenko AO, Podoplelova NA, Kolyadko VN, Chambost H, Mazurov AV, Ataullakhanov FI, Nurden AT, Alessi MC, Panteleev MA. Procoagulant platelets form an a-granule protein-covered "cap" on their surface that promotes their attachment to aggregates. J Biol Chem. 2013;288:29621-29632.

55. Mitchell JL, Lionikiene AS, Fraser SR, Whyte CS. Functional factor XIII-A is exposed on the stimulated platelet surface. Blood. 2014;124:3982-3990.

56. Kulkarni S, Jackson SP. Platelet factor XIII and calpain negatively regulate integrin $\alpha_{11 b} \beta_{3}$ adhesive function and thrombus growth. J Biol Chem. 2004;279:30697-30706.

57. Miles LA, Ginsberg MH, White JG, Plow EF. Plasminogen interacts with human platelets through two distinct mechanisms. J Clin Invest. 1986;77:2001-2009.

58. Soslau G, Class R, Morgan DA, Foster C, Lord ST, Marchese P, Ruggeri ZM. Unique pathway of thrombin-induced platelet aggregation mediated by glycoprotein Ib. J Biol Chem. 2001;276:21173- 
21183.

59. Plow EF, Collen D. The presence and release of a2-antiplasmin from human platelets. Blood. 1981;58:1069-1074.

60. Erickson LA, Ginsberg MH, Loskutoff DJ. Detection and partial characterization of an inhibitor of plasminogen activator in human platelets. $J$ Clin Invest. 1984;74:1465-1472.

61. Fay WP, Owen WG. Platelet plasminogen activator inhibitor: purification and characterization of interaction with plasminogen activators and activated protein C. Biochemistry. 1989;28:5773-5778.

62. Kruithof EK, Tran-Thang C, Bachmann F. Studies on the release of a plasminogen activator inhibitor by human platelets. Thromb Haemost. 1986;55:201-205.

63. Sprengers ED, Akkerman JW, Jansen BG. Blood platelet plasminogen activator inhibitor: two different pools of endothelial cell type plasminogen activator inhibitor in human blood. Thromb Haemost. 1986;55:325-329.

64. Wiman B, Collen D. On the kinetics of the reaction between human antiplasmin and plasmin. Eur J Biochem. 1978;84:573-578.

65. Hoylaerts M, Rijken DC, Lijnen HR, Collen D. Kinetics of the activation of plasminogen by human tissue plasminogen activator. Role of fibrin. J Biol Chem. 1982;257:2912-2919.

66. Stalker TJ, Welsh JD, Tomaiuolo M, Wu J, Colace TV, Diamond SL, Brass LF. A systems approach to hemostasis: 3. Thrombus consolidation regulates intrathrombus solute transport and local thrombin activity. Blood. 2014;124:1824-1831.

67. Wang X, Palasubramaniam J, Gkanatsas Y, Hohmann JD, Westein E, Kanojia R, Alt K, Huang D, Jia F, Ahrens I, Medcalf RL, Peter K, Hagemeyer CE. Towards effective and safe thrombolysis and thromboprophylaxis: preclinical testing of a novel antibody-targeted recombinant plasminogen activator directed against activated platelets. Circ Res. 2014;114:1083-1093. 


\section{Supplemental data}

Video 1. Formation of whole blood thrombi under flow. Thrombi were formed by whole blood perfusion $\left(1,000 \mathrm{~s}^{-1}\right)$ over a collagen/TF-coated surface for 7 minutes in the presence of tPA (30 nM). FibrinogenAF674 $\left(16.7 \mu \mathrm{g} / \mathrm{ml}\right.$; red) and $\mathrm{DIOC}_{6}$-labelled platelets $(0.5 \mu \mathrm{g} / \mathrm{ml}$; green). Consecutive images were acquired every 5 seconds, videos are at 2 frames $\mathrm{s}^{-1}$.

Video 2. Lysis of whole blood thrombi under flow with tPA (30 nM). Following thrombus formation ( $t=$ 0) lysis was monitored by perfusion $\left(1,000 \mathrm{~s}^{-1}\right)$ with Hepes buffer, $\mathrm{pH} 7.45$ containing $30 \mathrm{nM}$ tPA over time ( 8 minutes). Consecutive images were acquired every 5 seconds, videos are at 2 frames s$~^{-1}$.

A

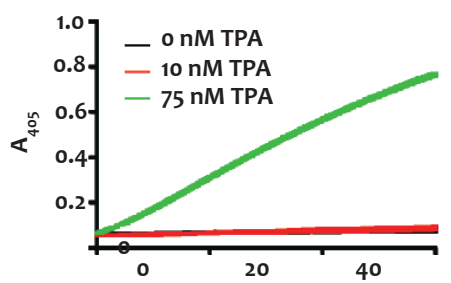

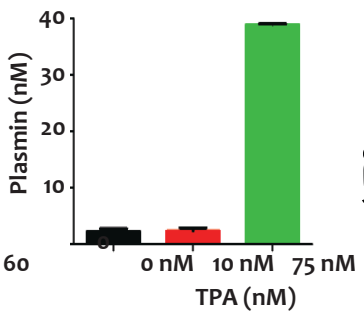

B Fgn $\frac{\text { plasma }}{0 \quad 1075 \mathrm{nM} \text { tPA }}$
C

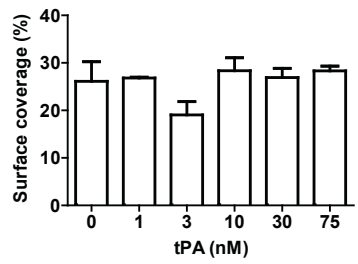

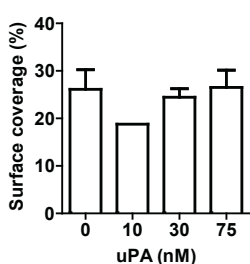

D

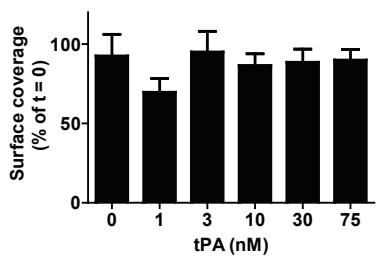

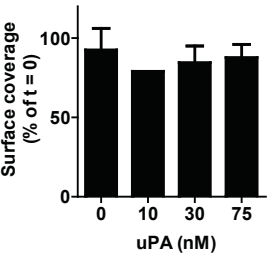

Supplemental Figure 1. tPA-mediated plasmin activity and surface coverage of DiOC $_{6}$-labelled platelets. Thrombi were formed by whole blood perfusion $\left(1,000 \mathrm{~s}^{-1}\right)$ over a collagen/TF-coated surface in the absence of presence of tPA or uPA (0-75 nM). Both fibrin(ogen)-OG488 and $\mathrm{DIOC}_{6}$, to label platelets, were added to the whole blood. After formation for 7 minutes thrombi were perfused with Hepes buffer, pH 7.45 for 8 minutes. In the absence of lysis, tPA or uPA was added to Hepes buffer, pH 7.45 for an additional 20 minutes $(t=28)(A)$ Effluents were collected during tPA-mediated fibrinolysis ( 0 , 10 and $75 \mathrm{nM}$ tPA) and plasmin activity quantified using the chromogenic substrate S2251 at $405 \mathrm{~nm}$. Plasmin generation shown as absorbance over time and plasmin concentration determined against a standard curve using average $A_{405} / \mathrm{min}$. ${ }^{* *} p<0.0001, n=2$. (B) Fibrinogenolysis with tPA was detected after incubating normal pooled human plasma was incubated \pm tPA 10 or $75 \mathrm{nM}$ for 30 minutes at 37 ${ }^{\circ} \mathrm{C}$. Degradation products were analysed using a peroxidase conjugated sheep anti-human fibrinogen antibody by Western Blotting. Purified human plasminogen-free fibrinogen (200 ng) was included as a control. (C) Percentage of total surface coverage with DiOC 6 -labelled platelets. (D) Surface coverage with $\mathrm{DiOC}_{6}$-labelled platelets as percentage of time $0 . n=3$. 



\title{
CHAPTER 9
}

\author{
General discussion
}


As stated in Chapter 1, haemostasis is the process that ensures the cessation of blood flow after tissue injury. It is well established now that activation of blood platelets as well as the coagulation system is required for a normal haemostatic response. ${ }^{1-3} \mathrm{~A}$ key interaction mechanism is known to be provided by exposure of the procoagulant phospholipid, phosphatidylserine (PS), at the surface of activated platelets, which allows generation of thrombin and formation of fibrin..$^{4-6}$ Yet, only little is understood of the many other molecular interactions between platelet and coagulation activation. The overall goal of the studies presented in this thesis was to investigate such interaction processes between platelet activation and coagulation under conditions of blood flow and relevant for haemostasis. One focus point was to evaluate the consequences of aberrant platelet signalling in haemostatic thrombus formation and thrombin generation in situ. Another focus point was to determine the role of von Willebrand factor (VWF) at the interface of platelet activation and coagulation. Furthermore, we aimed to understand the dynamic processes which feed thrombus formation and mediate degradation of the fibrin clot. As discussed below, the findings reported in this thesis may provide relevant new insights into how dysfunctions in either platelet or coagulant activity can lead to bleeding complications.

\section{Aberrant platelet signalling in procoagulant activity and aggregation}

Recent overviews summarise that platelets can be activated by both soluble and adhesive ligands via different sets of receptors. ${ }^{7-10}$ In the review Chapter 2, a comparison is made of the key platelet signalling events via G-protein-coupled receptors, interacting mostly with soluble agonists, and those via tyrosine kinaselinked receptors, specialised in transmitting responses due to binding of adhesive glycoproteins. We argue that the signalling pathways via both adhesive and soluble agonists are critical for normal haemostasis, as deficiencies in either can result in platelet dysfunction and bleeding complications. Importantly, impaired haemostasis also may result from acquired platelet function defects, for instance when the same receptors are used as drug targets to prevent cardiovascular disease, with minor or major bleeding as a not infrequent side effect. On the other hand diagnosis of patients with a congenital or acquired platelet function defect is not always easy, as such patients can present with only a mild bleeding phenotype. For the improvement of diagnosis, much gain is expected in the clinic from the development and use of new bleeding assessment tools, like detailed questionnaires and consistent registrations of all (subclinical) bleeding events. A questionnaire propagated by the International Society on Thrombosis and Haemostasis, developed for this purpose, is currently receiving much attention from the international community. ${ }^{11-13}$

In vitro whole blood flow assays of thrombus formation with murine blood have been shown to be of high predictive power for the assessment of in vivo arterial thrombotic tendencies. ${ }^{14}$ Accordingly, it is anticipated that the development of such flow assays can help in the detection of a bleeding or thrombotic tendency in 
patients. Hence, in the future, whole blood flow tests may be used as proxy assays for determining the (pathophysiological) roles of both platelets and the coagulation system in haemostasis. Yet, as will be described below, further standardisation and validation of the flow technique is required before this test can go into the clinic.

In Chapter 3 a study is performed to the role of Gsa in platelet signalling and function. In platelets and other cells, the GTP-binding Gsa protein (GNAS1 gene) is coupled to the IP and EP receptors for prostacyclin and prostaglandin $E_{1}$, stimulating adenylate cyclase $(A C)$ and thus providing a tonic levelling up of the cyclic AMP level. ${ }^{15}$, ${ }^{16}$ By activating the global serine/threonine protein kinase $A(P K A)$, cyclic AMP is known to act as a potent and overall inhibitor of platelet function. ${ }^{17}$ Hence, a deficiency in the Gsa/AC/PKA pathway is expected to result in increased residual activity of platelets, exposed to Gsa-activating stimuli. Patients with Albright hereditary osteodystrophy (AHO) carry a mutation in the GNAS1 gene, which leads to a hypo-function of the Gsa protein. $^{18,19}$ Most AHO patients however are heterozygous for the dysfunctional mutation, thus resulting in only partially reduced Gsa expression and activity, an effect that is usually measured in erythrocytes or fibroblasts. ${ }^{20-22}$ In the AHO patients also the penetration of disease is variable with respect to the pathophysiological symptoms, including osteodystrophy and mental retardation. So far, clinically relevant plateletrelated effects have only been observed in one patient suffering from a compound heterozygous Gsa defect (different mutations in either GNAS1 allele), in whom a 80$90 \%$ reduction in Gsa activity was accompanied with a prothrombotic tendency. ${ }^{23}$

In this chapter, we studied the responses of platelets to prostaglandin $\mathrm{E}_{1}$ or iloprost (i.e. a stable prostacyclin analogue) in two related patients diagnosed with $\mathrm{AHO}$. Whereas the platelets from healthy control subjects were consistently suppressed in aggregation upon Gsa stimulation, platelets from the AHO patients showed a significant residual platelet aggregation. In thrombi of aggregated platelets formed under flow conditions, this was accompanied by increased $\alpha_{1 \mathrm{lb}} \beta_{3}$ activation and granule secretion with Gsa-stimulating agents present. In earlier work with flow perfusion studies, our group has shown, using coagulating rat blood, that treatment with prostaglandin $E_{1}$ greatly impaired platelet aggregate formation, but only minimally decreased platelet phosphatidylserine (PS) exposure and fibrin formation. ${ }^{24}$ This suggested that in the presence of high thrombin generation the stimulation of Gsa inhibits the aggregatory function of platelets, yet maintains platelet-dependent coagulation via PS exposure. Summarising, it can be concluded that adequate Gsa signalling is required for a normal haemostatic response.

Based on recent progress in elucidation of the platelet proteome, ${ }^{25}$ we used in Chapter 3 highly sensitive mass spectrometric techniques to determine changes in the protein spectrum and phosphorylation profile of platelets from one of the AHO patients. We could identify 1,206 unique proteins in platelets, but without major differences in global proteome between the patient and a healthy control subject, in that $>95 \%$ of the proteins was unaltered in presence. Furthermore, in the Gsa-stimulated platelets (using iloprost) we identified phosphorylation sites in 3,457 peptides 


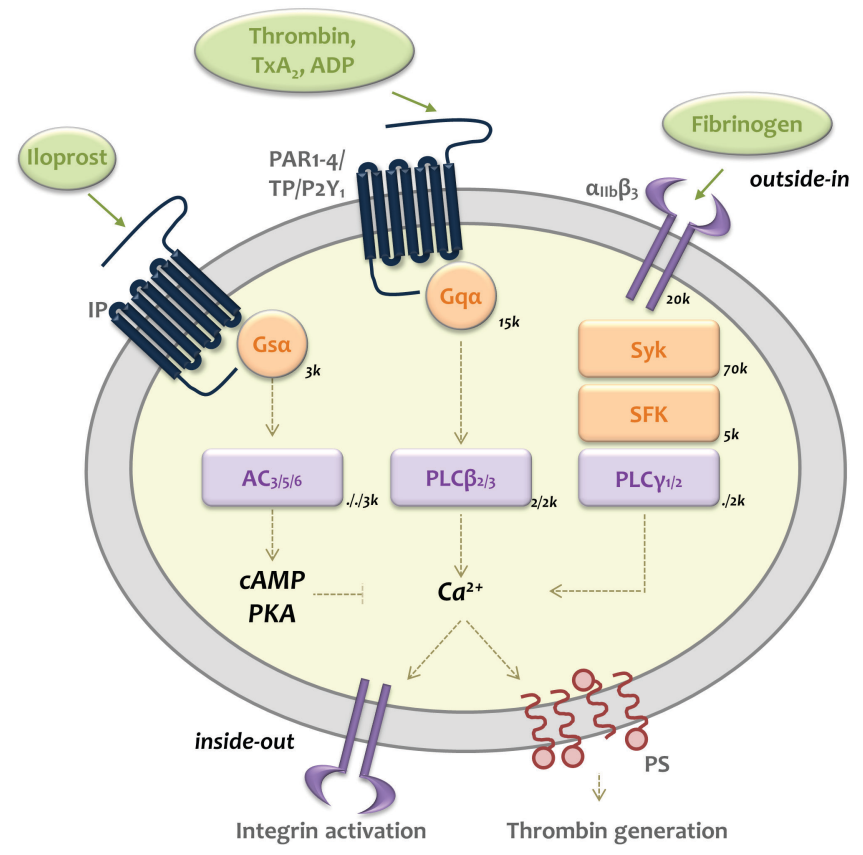

Figure 1. Signalling towards activation or inhibition of integrin $\alpha_{11 b} \beta_{3}$. Schematised are agonist-induced signalling pathways in platelets that are likely involved in normal haemostasis. Only key signalling proteins are indicated, as far as these contribute to the regulation of integrin $\alpha_{11 b} \beta_{3}$ activation and PS exposure and are discussed in this thesis. Protein isoforms are indicated in smaller font, while published copy numbers $(\times 1,000)$ per platelet are shown in italic. ${ }^{25}, 33$ For instance, for adenylate cyclase (AC), the indication $. / 1 / 3 \mathrm{k}$ indicates expression levels of 'unknown' (AC3/5) and $\sim 3,000$ (AC6) copy numbers per platelet. Dashed lines show networks of signals from proteins to the indicated platelet responses. See also text. Other abbreviations: Gsa, GTP-binding protein Gsa-subunit; SFK, Src family kinases; PLC, phospholipase C; PKA, protein kinase A (cyclic-AMP dependent protein kinase).

corresponding to 1,356 unique proteins. The iloprost-induced phosphoproteome of the patients showed 180 relevant changes of 239 phosphopeptides compared to control, half of which not identified earlier. Of these $84 \%$ concerned a decreased abundance in the AHO patients' platelets, in particular for phosphorylation sites that have previously been shown to be a target of cyclic AMP and PKA-dependent phosphorylation. ${ }^{17,26}$ In addition, we found a systematic lowering of iloprost-induced phosphorylation in platelets from the AHO patient in $82 \%$ of the newly recognised phosphopeptides regulated by iloprost. Mapping of the proteins with altered phosphorylation to platelet function classes indicated prominent roles, including signalling \& adapter proteins; protein kinases \& phosphatases and small GTPases \& regulators. Putative proteins with altered phosphorylation state in AHO platelets that can explain the decreased Gsa-induced inhibition of aggregation include phospholipase C- $\beta$ isoforms (genes PLCB2, PLCB3); type 3A phosphodiesterase (PDE3A); vasodilator-stimulated phosphoprotein (VASP); ${ }^{17}$ the inositol phosphate receptor regulator IRAG; ${ }^{26}$ and several members of the mitogen-activated protein kinase cascades (MINK1, MAP2K4, 
$M A P_{3} K_{2}$ ). Responsible for integrin $\alpha_{11 b} \beta_{3}$ activation and hence platelet aggregation via altered phosphorylation may especially be: the Rap1 controlling proteins, CalDAGGEFI (RASGRP2); ${ }^{27}$ and Rap1 GTPase-activating protein (RAP1GAP2); ${ }^{17}$ as well as the actin-linked proteins kindlin-3 (FERMT3), talin-1 (TLN1) and filamin A (FLNA). SNAREbinding proteins like tomosyn (STXPB5) are known to regulate granular secretion. ${ }^{28}$ Taken together, our data provide a detailed description of the altered Gsa-dependent functional responses of AHO platelets, i.e. integrin activation, granule secretion, activation, aggregation, and thrombus formation. Not only can platelet proteomics act as an important tool for determining the role of platelets in health and disease, but specifically the present phosphoproteomics is useful for diagnostic purposes in addition to understanding the molecular events regulating platelet inhibition via the key Gsa-AC-PKA signalling pathway.

Integrin $\alpha_{11 b} \beta_{3}$ is the most abundantly expressed of all glycoprotein receptors on the platelet surface (>70,000 copy numbers per cell). ${ }^{29}$ Once $\alpha_{11 \mathrm{~b}} \beta_{3}$ is activated by a conformational change, ligand binding to this integrin is known to provoke outside-in signalling events that mediate platelet responses such as spreading on a fibrinogen surface and retraction of a platelet-fibrin clot. ${ }^{30-32}$ In Chapter 5 we investigated whether $\alpha_{11} \beta_{3}$ outside-in signalling is also involved in the development of platelet procoagulant activity. This work was initiated by earlier reports describing anticoagulant effects of integrin $\alpha_{11 b} \beta_{3}$ antagonists on thrombin generation in plateletrich plasma. ${ }^{34-36}$ However, the underlying mechanism had not yet been determined. Here we report a pathway induced by $a_{11 b} \beta_{3}$ outside-in signalling that causes activation of the protein tyrosine kinase Syk, with as a consequence long-term intracellular $\mathrm{Ca}^{2+}$ rises and PS exposure (Fig. 1). Blockage of the integrin suppresses these processes and thereby reduces the generation of thrombin on the platelet surface. While the contribution of ITAM-bearing receptors like GPVI and CLEC-2 to Syk-dependent PS exposure had earlier been described in great detail, ${ }^{37-39}$ our findings are the first to demonstrate a similar activation pathway evoked by integrin $\alpha_{11 b} \beta_{3}$ outside-in signalling. In contrast to the present results, others did not detect inhibiting effects of integrin blockers on $\mathrm{Ca}^{2+}$ responses after collagen and thrombin stimulation..$^{40-42}$ However, these measurements concerned only initial $\mathrm{Ca}^{2+}$ rises that are unlikely to be mediated by integrin outside-in signalling. From the perspective of platelet control of the coagulation process, our results are interesting, because they implicate an intrinsic feedback mechanism. Earlier our group has shown that active $\alpha_{11 \mathrm{~b}} \beta_{3}$ integrins on PSexposing platelets become inactivated due to proteolytic cleavage. ${ }^{43}$ Together with the present results this implies that the $\alpha_{11 b} \beta_{3}$-mediated stimulation of PS exposure is followed by an inactivating pathway to abolish the integrin signalling. Considering the severe bleeding phenotype of Glanzmann patients lacking (functional) $\alpha_{11 b} \beta_{3},{ }^{44-46}$ our results further suggest that the procoagulant role of integrin signalling contributes to the impaired haemostasis in these patients. Taken together, it appears that $\alpha_{11 b} \beta$ has a star role in many intricate platelet activation processes, thus linking platelet aggregation, signalling and procoagulant activity. 


\section{Von Willebrand factor linking platelet and coagulation activation}

Together with platelet defects, deficiencies in VWF like in von Willebrand disease, represent the most common inherited bleeding disorders. ${ }^{47-49}$ This indicates that a certain activity of VWF is required for normal haemostasis. In Chapter 4, we hypothesised that this also applies to the bleeding risk of patients undergoing dilution during surgery. Support for this idea came from the use of desmopressin as a standard treatment option for patients experiencing post-operative bleeding after major surgery. Desmopressin is a vasopressin analogue that efficiently stimulates VWF release from the Weibel-Palade bodies in endothelial cells. ${ }^{50,51}$ We investigated the effects of desmopressin treatment in cardiothoracic patients with dilutional coagulopathy, who experienced major bleeding complications in the recovery room. Interestingly, irrespective of the high blood dilution, the pre-treatment levels of VWF in these patients were already high, which can be explained by endothelial stimulation due to the surgical intervention. ${ }^{52}$ Nevertheless, desmopressin treatment resulted in a further increase of in particular the high molecular weight multimers of VWF (Fig. 2A). This VWF rise was accompanied by a post-treatment increase in thrombus formation and PS exposure, when assayed in whole blood flowed at high wall shear rate, i.e. a condition known to be sensitive to VWF function. ${ }^{6}$ Our findings conform to the well-known clinical evidence that the haemostatic potential of VWF increases with its multimer size. ${ }^{53-55}$ Similarly, also other authors have reported that thrombus formation at high shear rate is dependent on the VWF level under conditions of haemodilution. ${ }^{56}$ In contrast to our observations, one study failed to detect positive effects of desmopressin treatment on thrombus formation. ${ }^{57}$ However, in that study flow assays were performed at low shear rate $\left(300 \mathrm{~s}^{-1}\right)$, where the contribution of (multimeric) VWF can be considered minimal. The observed increased PS exposure fits with findings from others that desmopressin treatment and, hence, VWF release promotes the formation of procoagulant, coated platelets. ${ }^{58}$ Hence, it can be concluded that the improved haemostatic state of patients receiving desmopressin is explained by the platelet-stimulating effect of released high molecular weight VWF multimers.

Several findings in this thesis also refer to another way how VWF can link platelet activation to coagulation. Chapter 4 describes that desmopressin treatment of patients with post-surgical bleeding, in addition to VWF, also increases the (already high) plasma level of factor VIII, which is in agreement with the known role of VWF as a carrier for factor VIII. Interestingly, we could not detect a coagulationpromoting effect using integrative coagulation assays, such as calibrated automated thrombin generation (CAT) or rotational thromboelastometry (ROTEM). This could be explained by the high pre-treatment levels of factor VIII (median $88 \%$ ), which likely were not rate-limiting for normal coagulation. In Chapter 7 we made the remarkable observation that, during the process of thrombus formation under coagulant conditions, immobilised VWF can serve as a capture pool for factor VIII. It appeared 
that this captured factor VIII was still available for tenase complex formation to interact with active factor IX, present on the PS-exposing platelets in a thrombus (Fig. 2A). Indirect support for the idea of factor VIII re-allocation comes from a recent report indicating that locally immobilised factor VIII is capable to enhance fibrin clot formation under flow. ${ }^{59}$

\section{Coagulation processes feeding thrombus and fibrin formation under flow conditions}

Thrombin, as a key product of the coagulation cascade and a main platelet activator, determines not only the rate and extent of fibrin formation, but also thrombus growth in vivo. ${ }^{60-62}$ The local thrombin concentration is regulated by the blood flow, which on the one hand facilitates the continuous supply of blood components (platelets, prothrombin, fibrinogen), and on the other hand mediates the removal of reaction products, e.g. thrombin complexes and fibrin monomers. ${ }^{63}$ Although there is no doubt that the interactions between platelets and coagulation are important in haemostasis, diagnostic testing for haemostatic defects is still performed with nonflow assays for either platelet function or coagulation activity. Some of the currently employed integrative tests - CAT and ROTEM - can be done with whole blood samples, ${ }^{64,65}$ but these are triggered in a way to principally detect coagulant rather than platelet activity. On the other hand, measurement of the procoagulant response (PS exposure) is an effective way to assess the procoagulant potential of platelets, ${ }^{2}$ but does not report on the coagulation process per se. In order to overcome the limitations of current haemostatic tests, we set to develop a flow assay incorporating the processes of platelet adhesion and aggregation, as well as those of thrombin generation and fibrin formation. By applying high shear flow conditions, the assay should be sensitive to VWF as well.

In earlier work it was established that collagen and tissue factor are key triggers of the formation of platelet-fibrin thrombi in vivo and in vitro., ${ }^{46}$ In Chapter 6, we determined how microspots with varied amounts of immobilised collagen and tissue factor influenced platelet adhesion, thrombus growth and the clotting potential under flow conditions. Confining the collagen content of a microspot specifically reduced initial platelet adhesion and ensuing platelet aggregation. However, it also appeared that the few thrombi formed on low collagen were relatively large in size due to a major contribution of tissue factor-triggered thrombin generation. This is in line with the platelet-stimulating effect of thrombin via PAR receptors ${ }^{61,67}$ and furthermore with in vivo findings showing that thrombin and fibrin accumulate in the core of a growing thrombus. ${ }^{68}$ We also introduced a novel method for investigating the elastic properties of the fibrin network formed, i.e. by nano-indentation. ${ }^{69,70}$ It appeared that especially the fibrin fibres located higher within thrombi contributed to elastic thrombus formation (Fig. 2A).

With optimised (high) amounts of collagen and tissue factor in microspots, we found that platelet adhesion, aggregation and PS exposure were all required for a 

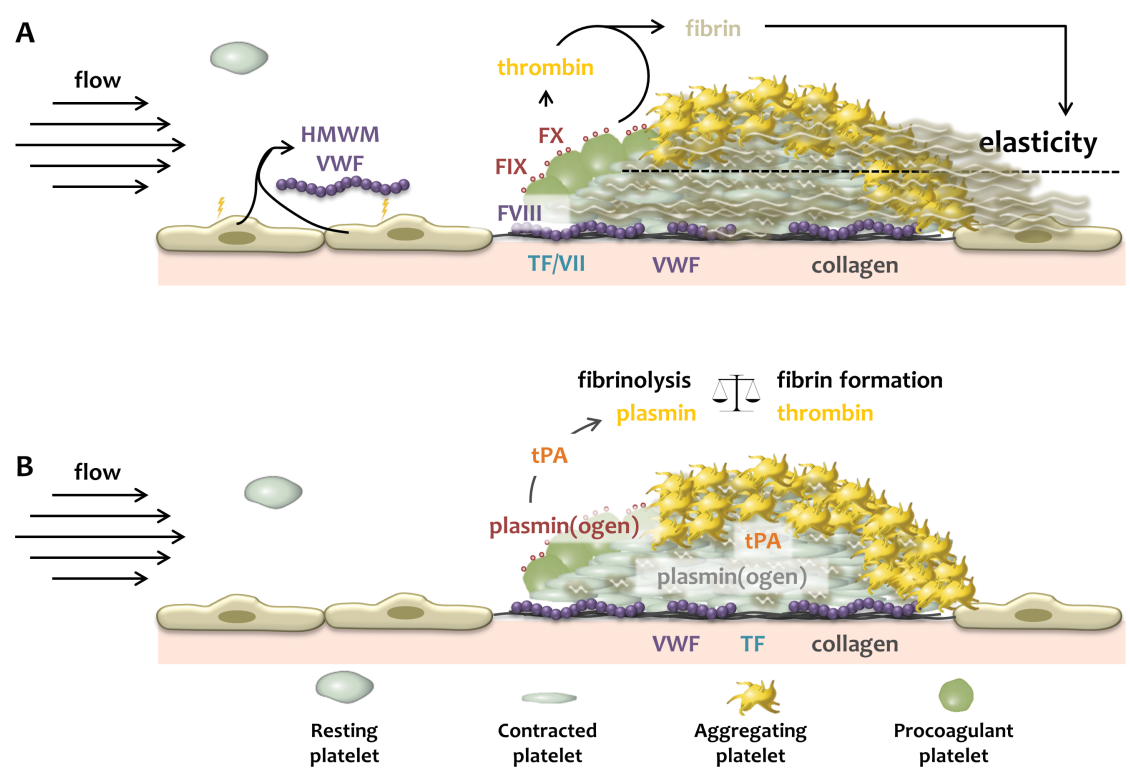

Figure 2. Schematic presentation of blood components critical for thrombus and fibrin formation and dissolution under flow conditions. (A) High molecular weight multimers (HMWM) of VWF promote thrombus formation and PS exposure. In addition, VWF serves as a capture pool for factor (F)VIII, which can be delivered to FIX on PS-exposing platelets for tenase complex formation, generating FXa and subsequently thrombin and fibrin. The elasticity of a thrombus relies on the presence of fibrin, especially in the top regions. (B) Plasminogen, binding to PS-exposing platelets, reallocates to fibrin fibres once these are formed. In the presence of tPA, plasmin is formed to induce fibrinolysis at a rate also dependent on the flow conditions. Furthermore, tPA incorporation in the growing thrombus enhances fibrinolysis in balance with the fibrin formation. See also text.

rapid onset of fibrin formation within and around the formed thrombi. Hence, fibrin formation depended on the presence of collagen as a platelet-capturing surface and the presence of tissue factor to stimulate thrombin generation (Fig. 2A). Accordingly, this is the preferred surface for a whole blood assay determining platelet activation and fibrin formation. Blood samples from various well-identified patients were tested under these conditions with microspots of high collagen and tissue factor. Deficiency in PS exposure (Scott syndrome patient), factor IX expression (haemophilia B), low platelet count (thrombocytopenia) or dilutional coagulopathy resulted in a greatly impaired fibrin formation under flow. The data hence highlight the relevance of platelet-coagulation interactions. Interestingly, a moderate decrease in platelet count (thrombocytopenic with a count of $85 \times 10^{9} / \mathrm{L}$ ) resulted in a delayed time to fibrin formation, with fibrin fibres located higher within thrombi. This is likely because a longer time period was needed to get sufficient platelet deposition and platelet coagulant activity for the clotting process to start.

In the studies of Chapter 7 we used a similar assay with mouse blood to determine the role of the tenase complex of factors VIII and IX in the formation of 
platelet-fibrin thrombi. From earlier work it was known that thrombin generation is enforced by a resonance loop with platelet activation, PS exposure and the cleavage of more prothrombin. ${ }^{38,66,71}$ How factor $X$ activation and PS exposure are linked has however remained unclear. Studies in the 1990-ies mostly confined to the assembly of the tenase complex factors on purified PS-containing phospholipid membranes. ${ }^{72,}$ 73 By performing whole blood flow studies under coagulant conditions, we could obtain detailed insight into the generation of factor Xa at a physiological thrombus. In mouse thrombi, we found factors IX and X both positioned on PS-exposing platelets, as expected from the presence of Gla-domains in these factors. It appeared that low amounts of the factor VIII(a) could be detected at the PS-exposing thrombi, but these were easily washed away. In contrast, the majority of the factor VIII staining after wash coincided with the staining pattern of VWF, co-localising with collagen and fibrin (Fig. 2A). Hence, we concluded that the VWF-bound factor VIII can act as a delivery site for the interaction with other tenase factors, as described above.

By flow perfusion experiments, in this setting with tissue factor added to the blood to 'saturate' the factor VIla pathway of coagulation, we established in Chapter 7 a role for factors VIII and IX in thrombus formation. These activate factor Xa and appear to cause a potent enforcement loop to subsequent thrombin generation, increased PS exposure, and resulting in fibrin formation. By implication, the pool of factor VIII that is bound at nearby VWF needs to relocate to the sites of factor X activation, likely after being cleaved into factor VIIla. Such an enforcing role of the tenase complex is supported by earlier studies, mostly using animal models, where deficiency in factor VIII or IX led to a diminished fibrin clot formation. ${ }^{74,75}$ Furthermore, also in mouse mouse models of arterial thrombosis both deficiencies greatly impaired thrombus growth and fibrin formation. ${ }^{76,77}$ With respect to human, other investigators monitored diminished thrombus formation and fibrin accumulation with blood from patients with haemophilia A (deficiency in factor VIII) or haemophilia B (deficiency in factor IX), but these concerned flow assays at low shear rate in the absence of tissue factor. ${ }^{78}$ Yet, it was concluded that residual factor activity determined the remaining fibrin-forming activity. In agreement with the lesser bleeding complication in haemophilia $B$ than in haemophilia $A,{ }^{78}$ we found in mouse that thrombus formation was less reduced with factor IX deficient blood, compared to factor VIII deficient blood.

\section{Dynamics of thrombus formation and fibrin clot lysis}

In the last decade, evidence has been accumulated that platelets play a role in the lysis of fibrin clots. Platelets can release from their a-granules various fibrinolytic proteins and inhibitors, including fibrinogen, plasminogen, plasminogen activator inhibitor-1 and $a_{2}$-antiplasmin. ${ }^{79-81}$ In Chapter 8 , we aimed to determine how the platelets in a fibrin-containing thrombus contributed to the lysis of fibrin fibres under conditions of flow. First, in the absence of fibrin fibres, we found plasminogen to be present on 
protruding 'caps' of PS-exposing platelets. This is in agreement with the literature that: (i) exposed PS on macrophages can act as a co-receptor for plasminogen together with histone $\mathrm{H}_{2} \mathrm{~B}$; ${ }^{82}$ and (ii) PS-exposing coated platelets accumulate also fibrin(ogen) in protruding caps. ${ }^{83-85}$ Subsequent experiments indicated that the binding of plasminogen to PS-exposing platelets was attenuated by the blocking of integrin $\alpha_{11 b} \beta_{3}$ and fibrin polymerisation. An earlier report indeed described that plasminogen binding is decreased for platelets from patients with Glanzmann's thrombasthenia. ${ }^{86}$ Overall, this points to an $\alpha_{11 b} \beta_{3}$-dependency of plasminogen binding to platelets via fibrin(ogen).

In thrombi generated from flowing whole blood, also in Chapter 8, we established that plasminogen mostly binds to platelet-connected fibrin fibres, with a smaller pool directly associating with platelets themselves. We further determined that the fibrin fibre degradation evoked by tissue or urokinase plasminogen activator (tPA or uPA) could be promoted by three factors: incorporation of the tPA/uPA into a growing thrombus, inhibition of thrombin and increased flow rates (Fig. 2B). The effect of tPA/uPA incorporation agrees with evidence from others that incorporation of the plasminogen activators in a large thrombus resulted in progressive lysis throughout the clot, thereby increasing plasminogen binding sites and promoting further lysis; whereas in contrast the addition of activators - as in thrombolytic therapies - resulted in superficial front lysis. ${ }^{87,88}$ With thrombin present, fibrin generation was ongoing and the fibrinolytic process appeared to be less effective. Another interesting finding was that the fibrin directly bound to the platelet surface was resistant to fibrinolysis. This is consistent with reports that the platelet-rich areas in a fibrin network form denser fibres, making these less sensitive to lytic degradation. ${ }^{89-91}$ Relevant in this context is further to mention that fibrin-bound plasminogen is protected from inhibition by $\alpha_{2}$ antiplasmin, ${ }^{92-94}$ implicating that the fibrin/plasminogen-rich thrombi are susceptible to fibrinolytic activity.

\section{Clinical implications}

Summarising, we have underscored in this thesis the importance of several interactions of platelets with the coagulation system, in experimental settings relevant for haemostasis. These interactions ranged from procoagulant consequences of platelet signalling pathways; and positive feedforward effects of tenase and prothrombinase on platelet activation; to the complex roles of platelets in fibrin formation and degradation. As listed in Table 1, all these interactions are of relevance for understanding the phenotype of patients with bleeding diatheses and explaining the bleeding side effects of antithrombotic drugs. A variety of platelet function tests is currently available (see Chapter 2), with light transmission aggregometry still as the gold standard, all with different sensitivities to specific platelet defects. ${ }^{95}$ Systematic use of these tests in combination with second generation sequencing has led to the identification of many novel mutations in genes encoding for relevant platelet 
proteins. ${ }^{96,97}$ Yet, the underlying (platelet) dysfunction in the majority of patients with mild bleeding complications remains unknown..$^{98}$ Thus, there is an ongoing need to develop new sensitive tools to register abnormalities in haemostatic platelet functions. Also with respect to coagulation, improved haemostatic tests are needed. For instance, it is not sufficient to measure individual factor levels to predict bleeding due to defective coagulation. Only one example of the many is the knowledge that two haemophilia A patients with equal factor VIII levels often do not experience the same bleeding tendency. ${ }^{99,} 100$

One future approach for testing normal or abnormal haemostasis is the further deployment of platelets proteomics, i.e. the global study of proteins expressed by a cell under well-defined conditions. ${ }^{101,102}$ An example in this thesis is the use of (phospho)proteomics for the phenotypic characterisation of an AHO patient with aberrant platelet signalling, which could be attributed to specific changes in the PKA-dependent protein phosphorylation pattern. Other examples of (phospho) proteomics analyses of platelets from patients with defined bleeding phenotypes are present in the recent literature. ${ }^{101}$ With the rapid progress in these techniques and the accompanying lower costs of such analyses - especially when carried out label-free - we foresee a more and more frequent use of phosphoproteomics as a diagnostic tool for detecting (unknown) platelet disorders. Furthermore, (phospho) proteomics analyses will help to understand the complex signalling mechanisms in platelet biology at normal and pathological conditions.

Obviously, in this thesis we also advocate another approach for the better predicting of haemostatic abnormalities. Since defective functions of platelets, VWF and the coagulation process independently can lead to bleeding, the whole blood flow assay measuring the formation of platelet-fibrin thrombi under high shear conditions, as described in this thesis, can serve as an integrative assay sensitive to all three haemostatic factors.

Table 1. Determinants of haemostasis, described in this thesis, that regulate the cross-talk between platelet and coagulation activation. Also indicated are the clinical relevance of the processes or factors, as apparent in the relevant chapters of this thesis.

\section{Blood process or factor}

Platelet integrin signalling (o/i, i/o)

Platelet procoagulant activity

Plasma VWF composition

Coagulation factors VIII, IX

Blood dilution

Fibrin formation

Fibrinolysis

\section{Clinical relevance for thesis}

Glanzmann patients, integrin blockers

Scott patient

Desmopressin treatment

Haemophilia patients

Perioperative bleeding

Perioperative bleeding, Scott patient, haemophilia patients

Tranexamic acid treatment

\section{Chapter}

$2,3,5$

3, 5,7

4, 7

7

4, 6

6, 7

8 


\section{References}

1. Monroe DM, Hoffman M. The clotting system - a major player in wound healing. Haemophilia. 2012;18 Suppl 5:11-16.

2. Heemskerk JW, Mattheij NJ, Cosemans JM. Platelet-based coagulation: different populations, different functions. J Thromb Haemost. 2013;11:2-16.

3. Cosemans JM, Angelillo-Scherrer A, Mattheij NJ, Heemskerk JW. The effects of arterial flow on platelet activation, thrombus growth, and stabilization. Cardiovasc Res. 2013;99:342-352.

4. Van der Meijden PE, Munnix IC, Auger JM, Govers-Riemslag JW, Cosemans JM, Kuijpers MJ, Spronk HM, Watson SP, Renné T, Heemskerk JW. Dual role of collagen in factor XII-dependent thrombus formation. Blood. 2009;114:881-890.

5. Cosemans JM, Schols SE, Stefanini L, de Witt S, Feijge MA, Hamulyák K, Deckmyn H, Bergmeier W, Heemskerk JW. Key role of glycoprotein Ib/V/IX and von Willebrand factor in platelet activationdependent fibrin formation at low shear flow. Blood. 2011;117:651-660.

6. De Witt SM, Swieringa F, Cavill R, Lamers MM, Van Kruchten R, Mastenbroek T, Baaten C, Coort S, Pugh N, Schulz A, Scharrer I, Jurk K, Zieger B, Clemetson KJ, Farndale RW, Heemskerk JW, Cosemans JM. Identification of platelet function defects by multi-parameter assessment of thrombus formation. Nat Commun. 2014;5:4257.

7. Jackson SP. The growing complexity of platelet aggregation. Blood. 2007;109:5087-5095.

8. Ruggeri ZM. Platelet adhesion under flow. Microcirculation. 2009;16:58-83.

9. Brass LF, Wannemacher KM, Ma P, Stalker TJ. Regulating thrombus growth and stability to achieve an optimal response to injury. J Thromb Haemost. 2011;9 Suppl 1:66-75.

10. Versteeg HH, Heemskerk JW, Levi M, Reitsma PH. New fundamentals in hemostasis. Physiol Rev. 2013;93:327-358.

11. Rodeghiero F, Tosetto A, Abshire T, Arnold DM, Coller B, James P, Neunert C, Lillicrap D. ISTH/SSC bleeding assessment tool: a standardized questionnaire and a proposal for a new bleeding score for inherited bleeding disorders. J Thromb Haemost. 2010;8:2063-2065.

12. Lowe GC, Lordkipanidze M, Watson SP. Utility of the ISTH bleeding assessment tool in predicting platelet defects in participants with suspected inherited platelet function disorders. J Thromb Haemost. 2013;11:1663-1668.

13. Deforest M, Grabell J, Albert S, Young J, Tuttle A, Hopman WM, James PD. Generation and optimization of the self-administered bleeding assessment tool and its validation as a screening test for von Willebrand disease. Haemophilia. 2015;21:e384-388.

14. De Witt S. The entanglement of thrombus formation: systems biology as a novel key. PhD Thesis, 2014, Maastricht University; Maastricht (The Netherlands).

15. Noe L, Peeters K, Izzi B, Van Geet C, Freson K. Regulators of platelet CAMP levels: clinical and therapeutic implications. Curr Med Chem. 2010;17:2897-2905.

16. Mischnik M, Hubertus K, Geiger J, Dandekar T, Timmer J. Dynamical modelling of prostaglandin signalling in platelets reveals individual receptor contributions and feedback properties. Mol Biosyst. 2013;9:2520-2529.

17. Smolenski A. Novel roles of CAMP/cGMP-dependent signaling in platelets. J Thromb Haemost. 2012;10:167-176.

18. Freson K, Izzi B, Labarque V, Van Helvoirt M, Thys C, Wittevrongel C, Bex M, Bouillon R, Godefroid N, Proesmans W, De Zegher F, Jaeken J, Van Geet C. GNAS defects identified by stimulatory G protein $\alpha$-subunit signalling studies in platelets. J Clin Endocrinol Metab. 2008;93:4851-4859.

19. Levine MA. An update on the clinical and molecular characteristics of pseudohypoparathyroidism. Curr Opin Endocrinol Diabetes Obes. 2012;19:443-451.

20. Levine MA, Downs RW, Jr., Singer M, Marx SJ, Aurbach GD, Spiegel AM. Deficient activity of guanine nucleotide regulatory protein in erythrocytes from patients with pseudohypoparathyroidism. 
Biochem Biophys Res Commun. 1980;94:1319-1324.

21. Levine MA, Eil C, Downs RW, Spiegel AM. Deficient guanine nucleotide regulatory unit activity in cultured fibroblast membranes from patients with pseudohypoparathyroidism type I. A cause of impaired synthesis of 3',5'-cyclic AMP by intact and broken cells. J Clin Invest. 1983;72:316-324.

22. Ahrens W, Hiort O. Determination of Gsa protein activity in Albright's hereditary osteodystrophy. J Pediatr Endocrinol Metab. 2006;19 Suppl 2:647-651.

23. Freson K, Izzi B, Jaeken J, Van Helvoirt M, Thys C, Wittevrongel C, De Zegher F, Van Geet C. Compound heterozygous mutations in the GNAS gene of a boy with morbid obesity, thyroidstimulating hormone resistance, pseudohypoparathyroidism, and a prothrombotic state. $J$ Clin Endocrinol Metab. 2008;93:4844-4849.

24. Kuijpers MJ, Nieuwenhuys CM, Feijge MA, Kloots W, Giesen PL, Jerling JC, Oude Egbrink MG, Heemskerk JW. Regulation of tissue factor-induced coagulation and platelet aggregation in flowing whole blood. Thromb Haemost. 2005;93:97-105.

25. Burkhart JM, Vaudel M, Gambaryan S, Radau S, Walter U, Martens L, Geiger J, Sickmann A, Zahedi RP. The first comprehensive and quantitative analysis of human platelet protein composition allows the comparative analysis of structural and functional pathways. Blood. 2012;120:e73-82.

26. Beck F, Geiger J, Gambaryan S, Veit J, Vaudel M, Nollau P, Kohlbacher O, Martens L, Walter U, Sickmann A, Zahedi RP. Time-resolved characterization of CAMP/PKA-dependent signaling reveals that platelet inhibition is a concerted process involving multiple signaling pathways. Blood. 2014;123:e1-e10.

27. Subramanian H, Zahedi RP, Sickmann A, Walter U, Gambaryan S. Phosphorylation of CalDAG-GEFI by protein kinase A prevents Rap1b activation. J Thromb Haemost. 2013;11:1574-1582.

28. GeneCards. www.gencards.org. 2015.

29. Fischer TH, Merricks EP, Bode AP, Bellinger DA, Russell K, Reddick R, Sanders WE, Nichols TC, Read MS. Thrombus formation with rehydrated, lyophilized platelets. Hematology. 2002;7:359-369.

30. Watson SP, Auger JM, McCarty OJ, Pearce AC. GPVI and integrin $\alpha_{11 b} \beta_{3}$ signaling in platelets. J Thromb Haemost. 2005;3:1752-1762.

31. Senis YA, Antrobus R, Severin S, Parguina AF, Rosa I, Zitzmann N, Watson SP, Garcia A. Proteomic analysis of integrin $\alpha_{11 b} \beta_{3}$ outside-in signaling reveals Src-kinase-independent phosphorylation of Dok-1 and Dok-3 leading to SHIP-1 interactions. J Thromb Haemost. 2009;7:1718-1726.

32. De Witt SM, Verdoold R, Cosemans JM, Heemskerk JW. Insights into platelet-based control of coagulation. Thromb Res. 2014;133 Suppl 2:S139-148.

33. Michelson AD. Platelets. Amsterdam: Elsevier. 2007.

34. Reverter JC, Béguin S, Kessels H, Kumar R, Hemker HC, Coller BS. Inhibition of platelet-mediated, tissue factor-induced thrombin generation by the mouse/human chimeric $7 \mathrm{E} 3$ antibody. Potential implications for the effect of C7E3 Fab treatment on acute thrombosis and "clinical restenosis". J Clin Invest. 1996;98:863-874.

35. Ilveskero S, Lassila R. Abciximab inhibits procoagulant activity but not the release reaction upon collagen- or clot-adherent platelets. J Thromb Haemost. 2003;1:805-813.

36. Vanschoonbeek K, Feijge MA, Van Kampen RJ, Kenis H, Hemker HC, Giesen PL, Heemskerk JW. Initiating and potentiating role of platelets in tissue factor-induced thrombin generation in the presence of plasma: subject-dependent variation in thrombogram characteristics. J Thromb Haemost. 2004;2:476-484.

37. Siljander PR, Munnix IC, Smethurst PA, Deckmyn H, Lindhout T, Ouwehand WH, Farndale RW, Heemskerk JW. Platelet receptor interplay regulates collagen-induced thrombus formation in flowing human blood. Blood. 2004;103:1333-1341.

38. Munnix IC, Kuijpers MJ, Auger J, Thomassen CM, Panizzi P, Van Zandvoort MA, Rosing J, Bock PE, Watson SP, Heemskerk JW. Segregation of platelet aggregatory and procoagulant microdomains in thrombus formation: regulation by transient integrin activation. Arterioscler Thromb Vasc Biol. 
2007;27:2484-2490.

39. Watson SP, Herbert JM, Pollitt AY. GPVI and CLEC-2 in hemostasis and vascular integrity. J Thromb Haemost. 2010;8:1456-1467.

40. Pedicord DL, Thomas BE, Mousa SA, Dicker IB. Glycoprotein IIb/IIla receptor antagonists inhibit the development of platelet procoagulant activity. Thromb Res. 1998;90:247-258.

41. Lages B, Weiss HJ. Greater inhibition of platelet procoagulant activity by antibody-derived glycoprotein Ilb-Illa inhibitors than by peptide and peptidomimetic inhibitors. $\mathrm{Br} J$ Haematol. 2001;113:65-71.

42. Razmara M, Hu H, Masquelier M, Li N. Glycoprotein Ilb/IIla blockade inhibits platelet aminophospholipid exposure by potentiating translocase and attenuating scramblase activity. Cell Mol Life Sci. 2007;64:999-1008.

43. Mattheij NJ, Gilio K, Van Kruchten R, Jobe SM, Wieschhaus AJ, Chishti AH, Collins P, Heemskerk JW, Cosemans JM. Dual mechanism of integrin $\alpha_{11 b} \beta_{3}$ closure in procoagulant platelets. J Biol Chem. 2013;288:13325-13336.

44. Hodivala-Dilke KM, McHugh KP, Tsakiris DA, Rayburn H, Crowley D, Ullman-Cullere M, Ross FP, Coller BS, Teitelbaum S, Hynes RO. $\beta_{3}$-integrin-deficient mice are a model for Glanzmann thrombasthenia showing placental defects and reduced survival. J Clin Invest. 1999;103:229-238.

45. Nurden AT, Fiore M, Nurden P, Pillois X. Glanzmann thrombasthenia: a review of ITCA2B and ITGB3 defects with emphasis on variants, phenotypic variability, and mouse models. Blood. 2011;118:59966005.

46. Tronik-Le Roux D, Roullot V, Poujol C, Kortulewski T, Nurden P, Marguerie G. Thrombasthenic mice generated by replacement of the integrin $\alpha_{11 b}$ gene: demonstration that transcriptional activation of this megakaryocytic locus precedes lineage commitment. Blood. 2000;96:1399-1408.

47. Sadler JE, Budde U, Eikenboom JC, Favaloro EJ, Hill FG, Holmberg L, Ingerslev J, Lee CA, Lillicrap D, Mannucci PM, Mazurier C, Meyer D, Nichols WL, Nishino M, Peake IR, Rodeghiero F, Schneppenheim R, Ruggeri ZM, Srivastava A, Montgomery RR, Federici AB. Update on the pathophysiology and classification of von Willebrand disease: a report of the Subcommittee on von Willebrand Factor. J Thromb Haemost. 2006;4:2103-2114.

48. Favaloro EJ, Bodo I, Israels SJ, Brown SA. von Willebrand disease and platelet disorders. Haemophilia. 2014;20 Suppl 4:59-64.

49. Lillicrap D. von Willebrand disease: advances in pathogenetic understanding, diagnosis, and therapy. Hematology Am Soc Hematol Educ Program. 2013;2013:254-260.

50. Ruggeri ZM, Mannucci PM, Lombardi R, Federici AB, Zimmerman TS. Multimeric composition of factor VIII/von Willebrand factor following administration of DDAVP: implications for pathophysiology and therapy of von Willebrand's disease subtypes. Blood. 1982;59:1272-1278.

51. Svensson PJ, Bergqvist PB, Juul KV, Berntorp E. Desmopressin in treatment of haematological disorders and in prevention of surgical bleeding. Blood Rev. 2014;28:95-102.

52. Panagiotopoulos I, Palatianos G, Michalopoulos A, Chatzigeorgiou A, Prapas S, Kamper EF. Alterations in biomarkers of endothelial function following on-pump coronary artery revascularization. J Clin Lab Anal. 2010;24:389-398.

53. Federici AB, Bader R, Pagani S, Colibretti ML, De Marco L, Mannucci PM. Binding of von Willebrand factor to glycoproteins Ib and IIb/IIla complex: affinity is related to multimeric size. $\mathrm{Br} J$ Haematol. 1989;73:93-99.

54. Fischer BE, Kramer G, Mitterer A, Grillberger L, Reiter M, Mundt W, Dorner F, Eibl J. Effect of multimerization of human and recombinant von Willebrand factor on platelet aggregation, binding to collagen and binding of coagulation factor VIII. Thromb Res. 1996;84:55-66.

55. Schneider SW, Nuschele S, Wixforth A, Gorzelanny C, Alexander-Katz A, Netz RR, Schneider MF. Shear-induced unfolding triggers adhesion of von Willebrand factor fibers. Proc Natl Acad Sci U S A. 2007;104:7899-7903. 
56. Ogawa S, Ohnishi T, Hosokawa K, Szlam F, Chen EP, Tanaka KA. Haemodilution-induced changes in coagulation and effects of haemostatic components under flow conditions. $\mathrm{Br} J$ Anaesth. 2013;111:1013-1023.

57. Calmer S, Ferkau A, Larmann J, Johanning K, Czaja E, Hagl C, Echtermeyer F, Goudeva L, Heuft HG, Theilmeier G. Desmopressin (DDAVP) improves recruitment of activated platelets to collagen but simultaneously increases platelet endothelial interactions in vitro. Platelets. 2014;25:8-15.

58. Colucci G, Stutz M, Rochat S, Conte T, Pavicic M, Reusser M, Giabbani E, Huynh A, Thurlemann C, Keller P, Alberio L. The effect of desmopressin on platelet function: a selective enhancement of procoagulant COAT platelets in patients with primary platelet function defects. Blood. 2014;123:1905-1916.

59. Doi M, Sugimoto M, Matsui H, Matsunari Y, Shima M. Coagulation potential of immobilised factor VIII in flow-dependent fibrin generation on platelet surfaces. Thromb Haemost. 2013;110:316-322.

60. Hathcock JJ, Nemerson Y. Platelet deposition inhibits tissue factor activity: in vitro clots are impermeable to factor Xa. Blood. 2004;104:123-127.

61. Okorie UM, Denney WS, Chatterjee MS, Neeves KB, Diamond SL. Determination of surface tissue factor thresholds that trigger coagulation at venous and arterial shear rates: amplification of 100 fM circulating tissue factor requires flow. Blood. 2008;111:3507-3513.

62. Colace TV, Muthard RW, Diamond SL. Thrombus growth and embolism on tissue factor-bearing collagen surfaces under flow: role of thrombin with and without fibrin. Arterioscler Thromb Vasc Biol. 2012;32:1466-1476.

63. Shen F, Kastrup CJ, Liu Y, Ismagilov RF. Threshold response of initiation of blood coagulation by tissue factor in patterned microfluidic capillaries is controlled by shear rate. Arterioscler Thromb Vasc Biol. 2008;28:2035-2041.

64. Ninivaggi M, Apitz-Castro R, Dargaud Y, De Laat B, Hemker HC, Lindhout T. Whole-blood thrombin generation monitored with a calibrated automated thrombogram-based assay. Clin Chem. 2012;58:1252-1259.

65. Lancé MD. A general review of major global coagulation assays: thrombelastography, thrombin generation test and clot waveform analysis. Thromb J. 2015;13:1.

66. Berny MA, Munnix IC, Auger JM, Schols SE, Cosemans JM, Panizzi P, Bock PE, Watson SP, McCarty OJ, Heemskerk JW. Spatial distribution of factor Xa, thrombin, and fibrin(ogen) on thrombi at venous shear. PLoS One. 2010;5:e10415.

67. Vu TK, Wheaton VI, Hung DT, Charo I, Coughlin SR. Domains specifying thrombin-receptor interaction. Nature. 1991;353:674-677.

68. Welsh JD, Colace TV, Muthard RW, Stalker TJ, Brass LF, Diamond SL. Platelet-targeting sensor reveals thrombin gradients within blood clots forming in microfluidic assays and in mouse. $J$ Thromb Haemost. 2012;10:2344-2353.

69. Chavan D, Andres D, lannuzzi D. Note: ferrule-top atomic force microscope. Il. Imaging in tapping mode and at low temperature. Rev Sci Instrum. 2011;82:046107.

70. Chavan D, Van de Watering TC, Gruca G, Rector JH, Heeck K, Slaman M, Iannuzzi D. Ferrule-top nanoindenter: an optomechanical fiber sensor for nanoindentation. Rev Sci Instrum. 2012;83:115110.

71. Beguin S, Kumar R. Thrombin, fibrin and platelets: a resonance loop in which von Willebrand factor is a necessary link. Thromb Haemost. 1997;78:590-594.

72. Lamphear BJ, Fay PJ. Factor IXa enhances reconstitution of factor VIIla from isolated A2 subunit and A1/A3-C1-C2 dimer. J Biol Chem. 1992;267:3725-3730.

73. Curtis JE, Helgerson SL, Parker ET, Lollar P. Isolation and characterization of thrombin-activated human factor VIII. J Biol Chem. 1994;269:6246-6251.

74. Hovig T, Dodds WJ, Rowsell HC, Mustard JF. The transformation of hemostatic platelet plugs in normal and Factor IX deficient dogs. Am J Pathol. 1968;53:355-373.

75. Vander Velden P, Giles AR. A detailed morphological evaluation of the evolution of the haemostatic 
plug in normal, factor VII and factor VIII deficient dogs. Br J Haematol. 1988;70:345-355.

76. Wang X, Cheng Q, Xu L, Feuerstein GZ, Hsu MY, Smith PL, Seiffert DA, Schumacher WA, Ogletree $M L$, Gailani D. Effects of factor IX or factor XI deficiency on ferric chloride-induced carotid artery occlusion in mice. $J$ Thromb Haemost. 2005;3:695-702.

77. Chauhan AK, Kisucka J, Lamb CB, Bergmeier W, Wagner DD. von Willebrand factor and factor VIII are independently required to form stable occlusive thrombi in injured veins. Blood. 2007;109:24242429.

78. Colace TV, Fogarty PF, Panckeri KA, Li R, Diamond SL. Microfluidic assay of hemophilic blood clotting: distinct deficits in platelet and fibrin deposition at low factor levels. J Thromb Haemost. 2014;12:147-158.

79. Coppinger JA, Cagney G, Toomey S, Kislinger T, Belton O, McRedmond JP, Cahill DJ, Emili A, Fitzgerald DJ, Maguire PB. Characterization of the proteins released from activated platelets leads to localization of novel platelet proteins in human atherosclerotic lesions. Blood. 2004;103:20962104.

80. Maynard DM, Heijnen HF, Horne MK, White JG, Gahl WA. Proteomic analysis of platelet a-granules using mass spectrometry. J Thromb Haemost. 2007;5:1945-1955.

81. Veljkovic DK, Rivard GE, Diamandis M, Blavignac J, Cramer-Borde EM, Hayward CP. Increased expression of urokinase plasminogen activator in Quebec platelet disorder is linked to megakaryocyte differentiation. Blood. 2009;113:1535-1542.

82. Das R, Plow EF. Phosphatidylserine as an anchor for plasminogen and its plasminogen receptor, histone H2B, to the macrophage surface. J Thromb Haemost. 2011;9:339-349.

83. Dale GL, Friese P, Batar P, Hamilton SF, Reed GL, Jackson KW, Clemetson KJ, Alberio L. Stimulated platelets use serotonin to enhance their retention of procoagulant proteins on the cell surface. Nature. 2002;415:175-179.

84. Dale GL. Coated-platelets: an emerging component of the procoagulant response. J Thromb Haemost. 2005;3:2185-2192.

85. Abaeva AA, Canault M, Kotova YN, Obydennyy SI, Yakimenko AO, Podoplelova NA, Kolyadko VN, Chambost H, Mazurov AV, Ataullakhanov FI, Nurden AT, Alessi MC, Panteleev MA. Procoagulant platelets form an a-granule protein-covered cap on their surface that promotes their attachment to aggregates. J Biol Chem. 2013;288:29621-29632.

86. Miles LA, Ginsberg MH, White JG, Plow EF. Plasminogen interacts with human platelets through two distinct mechanisms. J Clin Invest. 1986;77:2001-2009.

87. Sakharov DV, Rijken DC. Superficial accumulation of plasminogen during plasma clot lysis. Circulation. 1995;92:1883-1890.

88. Sakharov DV, Nagelkerke JF, Rijken DC. Rearrangements of the fibrin network and spatial distribution of fibrinolytic components during plasma clot lysis. Study with confocal microscopy. J Biol Chem. 1996;271:2133-2138.

89. Gabriel DA, Muga K, Boothroyd EM. The effect of fibrin structure on fibrinolysis. J Biol Chem. 1992;267:24259-24263.

90. Collet JP, Montalescot G, Lesty C, Weisel JW. A structural and dynamic investigation of the facilitating effect of glycoprotein IIb/IIla inhibitors in dissolving platelet-rich clots. Circ Res. 2002;90:428-434.

91. Collet JP, Park D, Lesty C, Soria J, Soria C, Montalescot G, Weisel JW. Influence of fibrin network conformation and fibrin fiber diameter on fibrinolysis speed: dynamic and structural approaches by confocal microscopy. Arterioscler Thromb Vasc Biol. 2000;20:1354-1361.

92. Hall SW, Humphries JE, Gonias SL. Inhibition of cell surface receptor-bound plasmin by $a_{2}{ }^{-}$ antiplasmin and $\alpha_{2}$-macroglobulin. J Biol Chem. 1991;266:12329-12336.

93. Plow EF, Freaney DE, Plescia J, Miles LA. The plasminogen system and cell surfaces: evidence for plasminogen and urokinase receptors on the same cell type. J Cell Biol. 1986;103:2411-2420. 
94. Wiman B, Collen D. On the kinetics of the reaction between human antiplasmin and plasmin. Eur J Biochem. 1978;84:573-578.

95. Gresele P, Harrison P, Bury L, Falcinelli E, Gachet C, Hayward CP, Kenny D, Mezzano D, Mumford $A D$, Nugent D, Nurden AT, Orsini S, Cattaneo M. Diagnosis of suspected inherited platelet function disorders: results of a worldwide survey. J Thromb Haemost. 2014;12:1562-1569.

96. Westbury SK, Turro E, Greene D, Lentaigne C, Kelly AM, Bariana TK, Simeoni I, Pillois X, Attwood A, Austin S, Jansen SB, Bakchoul T, Crisp-Hihn A, Erber WN, Favier R, Foad N, Gattens M, Jolley JD, Liesner R, Meacham S, Millar CM, Nurden AT, Peerlinck K, Perry DJ, Poudel P, Schulman S, Schulze H, Stephens JC, Furie B, Robinson PN, Van Geet C, Rendon A, Gomez K, Laffan MA, Lambert MP, Nurden P, Ouwehand WH, Richardson S, Mumford AD, Freson K. Human phenotype ontology annotation and cluster analysis to unravel genetic defects in 707 cases with unexplained bleeding and platelet disorders. Genome Med. 2015;7:36.

97. Leo VC, Morgan NV, Bem D, Jones ML, Lowe GC, Lordkipanidze M, Drake S, Simpson MA, Gissen P, Mumford A, Watson SP, Daly ME. Use of next-generation sequencing and candidate gene analysis to identify underlying defects in patients with inherited platelet function disorders. $J$ Thromb Haemost. 2015;13:643-650.

98. Watson SP, Lowe GC, Lordkipanidze M, Morgan NV. Genotyping and phenotyping of platelet function disorders. J Thromb Haemost. 2013;11 Suppl 1:351-363.

99. Santagostino E, Mancuso ME, Tripodi A, Chantarangkul V, Clerici M, Garagiola I, Mannucci PM. Severe hemophilia with mild bleeding phenotype: molecular characterization and global coagulation profile. J Thromb Haemost. 2010;8:737-743.

100. Carcao MD, Van den Berg HM, Ljung R, Mancuso ME. Correlation between phenotype and genotype in a large unselected cohort of children with severe hemophilia A. Blood. 2013;121:3946-3952, s3941.

101. Di Michele M, Van Geet C, Freson K. Proteomics to unravel platelet-related diseases and identify novel anti-platelet drugs. Curr Med Chem. 2012;19:4662-4670.

102. Di Michele M, Van Geet C, Freson K. Recent advances in platelet proteomics. Expert Rev Proteomics. 2012;9:451-466. 


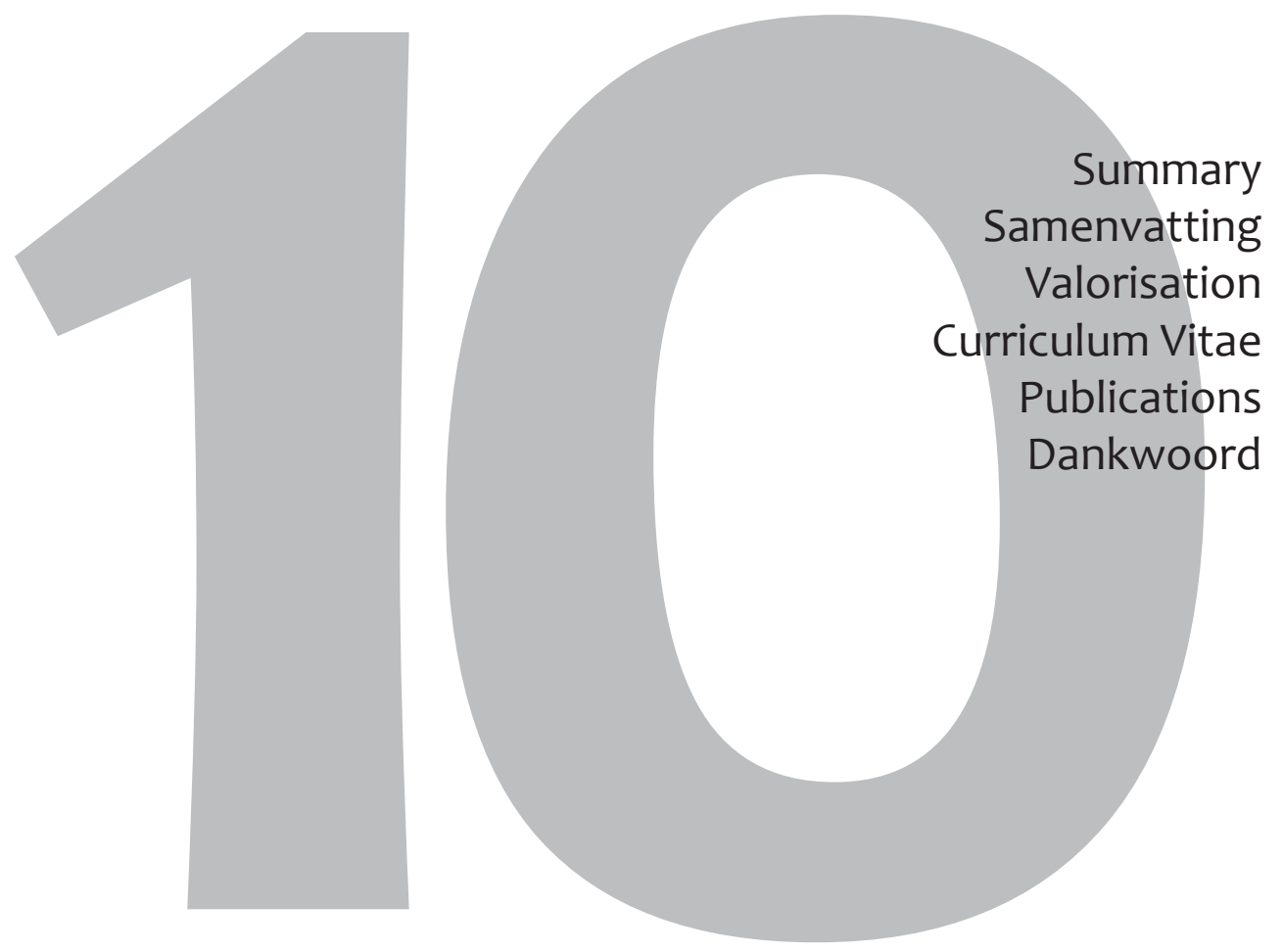

Summary 


\section{Platelets and coagulation: partners in haemostasis}

Haemostasis is the process that ensures cessation of blood flow after tissue injury. It relies on the activation of both platelets and the coagulation cascade, and results in the formation of a platelet-fibrin thrombus or clot. Functional defects in these processes can lead to a decreased thrombus/clot forming potential and bleeding. The contribution of platelets to haemostasis is not limited to their adhesive and cohesive functions, but extends to their ability to stimulate the coagulation process. Exposed phosphatidylserine on the surface of activated platelets acts as a site for the assembly of coagulation factors, which catalyse several proteolytic steps in the coagulation cascade, in particular causing activation of factor $\mathrm{X}$ into factor $\mathrm{Xa}$ and prothrombin into thrombin. The studies in this thesis were performed to identify novel links between platelet activation and the formation and breakdown of a fibrin clot. Chapter 1 provides a brief, general background on the known mechanisms of platelet activation and coagulation activity in thrombus formation. Highlighted here are key platelet receptors in clot formation; the use of proteomics to identify the roles of platelet protein kinases; the regulation of thrombin generation, fibrin formation and fibrinolysis; and description of the multiple interactions of platelets and coagulation in thrombus formation.

Presuming that the process of thrombus formation, as assessed by whole blood flow experiments and experimental thrombosis studies, adequately reflects the platelet responses in haemostasis, in Chapter $\mathbf{2}$ an extended overview is given on the main platelet receptors and downstream signalling pathways that are implicated in haemostasis. In addition, potential therapeutic targets are discussed of these platelet activation pathways. Currently, the main receptors with potential or established clinical relevance are those of thrombin, thromboxane $A_{2}, A D P, A T P$, prostaglandins, von Willebrand factor (VWF), collagen, CLEC-2 ligand, fibrinogen and laminin. Dysfunction of one or more of these platelet activation pathways, which can be either inherited or acquired due to antiplatelet medication, may lead to a higher risk of bleeding. Only mild bleeding complications are reported in case of deficiency in the collagen receptor glycoprotein $\mathrm{VI}$, making this signalling pathway an interesting target for antithrombotic therapy. It is also described that, for proper diagnosis and treatment, uniformity is needed in the registration of bleeding events, e.g. by using standardised assessment tools and platelet function tests.

In Chapter 3 sensitive (phospho)proteomics approaches are used as a novel fascinating technique to study platelet function defects. In patients with Albright hereditary osteodystrophy (AHO), linked to a heterozygous mutation in the GNAS1 gene cluster, a hypofunction exists of the GTP-bound Gsa protein, resulting in impaired signalling via adenylate cyclase (AC) and CAMP-dependent protein kinase $A$ (PKA). The Gsa protein is activated by the IP receptor for prostacyclin and iloprost. By using highly sensitive quantitative techniques, changes in protein abundance and protein phosphorylation pattern were assessed in platelets from a well-identified 
AHO patient. While the global proteome was not different from that of healthy controls, phosphoproteomic analysis revealed 180 altered protein phosphorylation sites along the Gsa-AC-PKA axis in AHO platelets stimulated with iloprost. These 180 phosphorylation events were particularly found in protein targets downstream of PKA; approximately $50 \%$ of these were novel phosphorylation sites that have not been described before. The altered, decreased, phosphorylation state in AHO platelets reflects the impaired ability of iloprost to inhibit platelet responses, such as aggregation, secretion and thrombus formation.

Thrombus formation at high shear rate is initiated by platelet adhesion to VWF bound to collagen fibres via the receptor complex, glycoprotein Ib-V-IX. In Chapter 4, we supposed that cardiothoracic patients who suffer from postoperative bleeding complications due to haemodilution benefit from desmopressin treatment due to do novo release of VWF from the endothelium. We found that the plasma levels of VWF were already high in these patients pretreatment, but that desmopressin further increased the levels in particular of the active, high molecular weight VWF multimers. This increase in large VWF multimers resulted in an improved platelet deposition and thrombus formation on collagen under flow, as well as in enhanced platelet procoagulant activity (phosphatidylserine exposure). No major effect of desmopressin was found on the coagulant state of plasma, as determined by thrombin generation and thromboelastometry measurements. The results suggest that an increased VWF-dependent platelet activity can help to restore haemostasis in these bleeding patients.

With fibrinogen as a main ligand, integrin $\alpha_{111} \beta_{3}$ plays an important role in the development of platelet procoagulant activity, in addition to its classical role in platelet aggregate formation. In Chapter 5 we identified an $\alpha_{\mathrm{Ib}} \beta_{3}$ outside-in signalling pathway that stimulates the late phase of the $\mathrm{Ca}^{2+}$ responses in platelets, thereby increasing phosphatidylserine exposure and enforcing tissue factor-triggered thrombin generation on platelets. In agreement with inhibitor studies, we found that platelets from patients with Glanzmann's thrombasthenia, lacking $\alpha_{11 b} \beta_{3}$, possessed a reduced procoagulant potential. Analysis of the protein tyrosine phosphorylation pattern pointed to a key role of the kinase Syk, which was largely but not exclusively dependent on $\alpha_{11 b} \beta_{3}$ activation.

In Chapter 6 we developed and employed an integrative whole blood flow method, allowing the combined assessment of platelet and coagulant functions. This allowed us to assess the formation and composition of fibrin clots on various thrombogenic surfaces consisting of collagen and tissue factor. Lowering the collagen content reduced platelet deposition and fibrin formation, which effects were compensated by a larger thrombus size and an increased accumulation of fibrin in the luminal regions of the thrombi. At low collagen, the formation of thrombi was more dependent on the local generation of thrombin. In addition, we used a novel nano-indentation technique, to assess the mechanical properties - i.e. the microelasticity - of thrombi formed on these physiological surfaces. We observed that the redistribution of fibrin 
from the base to the luminal region of a thrombus was accompanied by an increased microelasticity. This redistribution was also observed with thrombocytopenic blood, where not the collagen content on the surface but the platelet count is a limiting factor in fibrin clot formation. These findings demonstrated that, under conditions of flow and coagulation, the amount and localisation of fibrin are dependent on the relative abundance of the triggers for platelet adhesion (collagen) and coagulation (tissue factor). Furthermore, the microelasticity of a thrombus appears to be dependent on the fibrin distribution in the thrombus.

In Chapter 7 we assessed how the intrinsic coagulation pathway to tenase activation contributes to the formation of platelet-fibrin thrombi under flow. While there is strong experimental evidence that phosphatidylserine-exposing platelets in a thrombus support prothrombinase activity, the exact mechanism how factor $X$ activation on a thrombus is regulated is much less understood. We found that activated factor IX and $X$ are located on phosphatidylserine-exposing positive platelets in a thrombus, whereas factor VIII co-localises with VWF, which in turn is bound to collagen and fibrin fibres. The results point to a mechanism of delivery of VWF-bound factor VIII to phosphatidylserine-exposing platelets, resulting in transient formation of the tenase complex, which then serves to enhance thrombus formation. Perfusion experiments with human and mouse blood with deficiencies in factor VIII or IX indicated a limiting role for both factors in the factor Xa-mediated stimulation of platelet procoagulant activity via thrombin generation and subsequent fibrin formation. Whereas haemophilia A and B are often considered as similar pathologies, our data indicated that the formation of platelet-fibrin thrombi was less reduced with factor IX-deficient blood than with factor VIII-deficient blood. This might explain the lesser bleeding complications in patients with factor IX deficiency.

Additionally to controlling thrombus and fibrin formation, platelets can also play a role in fibrinolysis as they secrete the fibrinolytic factor plasminogen from their a-granules. In Chapter 8, we investigated how a platelet-fibrin thrombus is lysed under flow conditions, with particular focus on the role of plasminogen. Highresolution microscopy indicated that plasminogen is localised on 'caps' protruding from phosphatidylserine-exposing platelets. The binding of plasminogen relies on integrin $\alpha_{11 b} \beta_{3}$ and fibrin(ogen), as blocking of either $\alpha_{11 b} \beta_{3}$ or fibrin polymerisation attenuated this interaction. In thrombi formed by whole blood perfusion plasminogen primarily bound to platelet-associated fibrin, with a smaller pool directly binding to platelets. Degradation of fibrin in the thrombi was dose-dependently induced by tissue and urokinase plasminogen activator (tPA/uPA), and was promoted by three distinct factors, namely by: incorporation of tPA/uPA into the growing thrombus, inhibition of (thrombin and) fibrin formation, and increasing the flow rate.

The final Chapter 9 discusses the key findings of this thesis in the light of relevant literature. New insights into the underlying mechanisms of thrombus formation reveal intricate interactions between platelet activation and coagulant activity, and help to better understand bleeding complications in patients in various clinical settings. 

Samenvatting 


\section{Plaatjes en stolling: partners in hemostase}

Hemostase is het proces dat zorgt voor het stelpen van een bloeding, als gevolg van beschadiging van een bloedvat. Deze bloedstelping is afhankelijk van de activering van bloedplaatjes en de stollingscascade, en leidt tot de vorming van een plaatjesfibrine trombus of stolsel. Functionele defecten in deze activeringsprocessen kunnen leiden tot een verminderde potentie om een stolsel te vormen met een mogelijke bloeding als gevolg. De bijdrage van bloedplaatjes aan hemostase is niet beperkt tot hun adhesieve en cohesieve eigenschappen, maar omvat ook hun vermogen om het stollingsproces sterk te stimuleren. Op geëxposeerd fosfatidylserine aanwezig op de buitenmembraan van geactiveerde bloedplaatjes hechten diverse stollingsfactoren, hetgeen leidt tot een katalyse van verschillende proteolytische stappen in de stollingscascade, in het bijzonder de activering van factor $\mathrm{X}$ naar factor Xa en van protrombine naar trombine. Echter er zijn vele aanwijzingen dat er ook andere interacties zijn tussen de activering van bloedplaatjes en de stollingscascade. De studies in dit proefschrift zijn uitgevoerd om nieuwe schakels tussen plaatjesactivering en de vorming en afbraak van een fibrine-stolsel te identificeren en op te helderen. Hoofdstuk $\mathbf{1}$ biedt hiertoe een korte, algemene achtergrond over de tot dusver bekende mechanismen van plaatjesactivering en stollingsactiviteit bij de trombusvorming. Uitgelicht zijn hier de belangrijkste betrokken plaatjesreceptoren; het mogelijk gebruik van proteomics-technieken om de meest relevante eiwitten te identificeren; op hoofdlijnen de regulatie van trombinegeneratie, fibrinevorming en fibrinolyse; en de beschrijving van bekende interactie-mechanismen.

Gebaseerd op het concept dat het proces van trombusvorming, zoals dat plaatsvindt in volbloed perfusie-experimenten en in vivo trombosestudies in diermodellen, een adequaat inzicht biedt in de bloedplaatjes-reacties bij hemostase, biedt Hoofdstuk 2 een uitgebreid overzicht van de belangrijkste receptoren en de bijbehorende signaleringspaden die betrokken zijn bij het proces van trombusvorming. Daarnaast worden potentiële therapeutische doeleiwitten in deze signaleringspaden besproken. Voor de kliniek zijn de belangrijkste receptoren met potentiële of bekende relevantie de receptoren voor trombine, tromboxaan $A_{2}$, ADP, ATP, prostaglandines, von Willebrand factor (VWF), collageen, CLEC-2 ligand, fibrinogeen en laminine. Dysfunctie van één of meer van deze receptoren of betrokken signaleringspaden, door oftewel een genetisch defect oftewel een verworven defect middels antiplaatjesmedicatie, kan leiden tot een verhoogd bloedingsrisico. Enkel milde bloedingscomplicaties zijn bekend in geval van een deficiëntie van de collageenreceptor glycoproteïne $\mathrm{VI}$, hetgeen deze receptor tot een interessant doeleiwit maakt voor antitrombotische therapie. Ook is in dit hoofdstuk beschreven, dat voor een juiste diagnose en behandeling uniformiteit nodig is bij de registratie van bloedingsepisodes, alsmede het belang van gestandaardiseerde diagnostische instrumenten en gevalideerde plaatjesfunctietesten.

In Hoofdstuk 3 is gebruik gemaakt van geavanceerde (fosfo)proteomics 
technieken als een nieuwe, fascinerende methode om structurele en functiedefecten van bloedplaatjes te bestuderen. In patiënten met Albright hereditary osteodystrophy $(\mathrm{AHO})$, gelinkt aan een heterozygote mutatie in het GNAS1 gen-cluster, is er sprake van een hypofunctie van het GTP-gebonden eiwit Gsa. Dit resulteert in een beperkte signalering via adenylaat cyclase $(A C)$ en het CAMP-afhankelijke protein-kinase $A$ (PKA). In bloedplaatjes wordt het Gsa eiwit geactiveerd door de IP-receptor voor prostacycline en iloprost. Door gebruik te maken van zeer gevoelige kwantitatieve technieken, konden we de eiwit-patronen en fosforyleringsstatus bepalen in iloprostbehandelde bloedplaatjes van een goed gekarakteriseerde AHO patiënt. Hoewel het globale proteoom niet verschilde van dat van controle-bloedplaatjes, toonde de fosfoproteomics analyse aan dat er 180 fosforyleringsplaatsen in eiwitten veranderend waren in AHO plaatjes gestimuleerd met iloprost. Veranderde fosforylering vond voornamelijk plaats in potentiële PKA substraten (de signaleringsas Gsa-AC-PKA). Ongeveer 50\% van deze waren nieuw geïdentificeerde fosforyleringsplaatsen, die niet eerder in de literatuur beschreven zijn. De verminderde fosforyleringsstatus van AHOplaatjes reflecteerde de verminderde capaciteit van iloprost om bloedplaatjesfuncties zoals aggregatie, secretie en trombusvorming te onderdrukken.

Trombusvorming bij een hoge stroomsnelheid van het bloed wordt geïnitieerd door de aanhechting van bloedplaatjes aan VWF dat bindt aan collageenvezels via het receptorcomplex glycoproteïne Ib-V-IX. In Hoofdstuk 4 hebben we onderzocht of cardio-thoracale patiënten die lijden aan postoperatieve bloedingscomplicaties als gevolg van bloedverdunning, profiteren van behandeling met desmopressine door de vrijlating van VWF vanuit het endotheel. We vonden dat in deze patiënten de plasmaspiegels van VWF voor de behandeling reeds hoog waren, maar dat na desmopressine deze niveaus nog verder toenamen, met name van de meest actieve hoog molecuulgewicht multimeren van VWF. Deze toename in grote VWFmultimeren resulteerde in een verbeterde plaatjesdepositie en trombusvorming op collageen onder stromingscondities, en eveneens in een verhoogde stollingsactiviteit van bloedplaatjes (verhoogde fosfatidylserine-expositie). Daarentegen konden we geen effect vinden van desmopressine-behandeling op de stollingscapaciteit van het bloedplasma, zoals bepaald middels metingen van trombinegeneratie en tromboelastometrie. Deze resultaten suggereren dat een verhoogde VWFafhankelijke plaatjesactiviteit kan bijdragen tot de herstelde hemostase in deze patiënten met postoperatieve bloedingen.

Met fibrinogeen als belangrijkste ligand, speelt integrine $\alpha_{111} \beta_{3}$ een rol in het ontstaan van procoagulante activiteit van bloedplaatjes, naast de klassieke rol in vorming van plaatjesaggregaten. In Hoofdstuk 5 beschrijf ik de identificatie van een integrine $a_{\| b} \beta_{3}$-afhankelijk outside-in signaleringspad, dat bijdraagt tot de calciumrespons van bloedplaatjes, daarmee de fosfatidylserine-expositie verhoogt en de trombinegeneratie onder invloed van weefselfactor op deze bloedplaatjes versterkt.Zowel onderinvloed van integrine-remmers alsinbloedplaatjes van patiënten met Glanzmann's thrombasthenia (deficiëntie in $\alpha_{11} \beta_{3}$ ) maten wij een verminderde 
procoagulante activiteit. Analyse van het tyrosine eiwitfosforyleringspatroon wees hierbij op een kern rol van Syk-kinase, welke grotendeels maar niet exclusief afhankelijk was van $\alpha_{1 \mathrm{lb}} \beta_{3}$-activering.

In Hoofdstuk 6 heb ik een integratieve volbloedmeting ontwikkeld en gebruikt, waarmee het mogelijk wordt tegelijkertijd de bijdrage van bloedplaatjes en bloedstolling te meten in de trombusvorming onder stromingscondities. Deze methode stelde ons in staat om de vorming en samenstelling te bepalen van plaatjesfibrine trombi op verschillende trombogene oppervlakken, bestaande uit collageen en weefselfactor. Een lagere collageendichtheid leidde tot een verminderde plaatjesdepositie en fibrinevorming, hetgeen gecompenseerd werd door een toegenomen trombusgrootte en accumulatie van fibrine aan de bovenzijde van de trombus. Bij lage collageendichtheden werd de vorming van trombi bovendien meer afhankelijk van lokaal gegenereerd trombine. Daarnaast hebben we gebruik gemaakt van we een nieuwe nanoindentatie-methode om beter inzicht te krijgen in de mechano-elastische eigenschappen (microelasticiteit) van trombi gevormd op deze fysiologische oppervlakken. We vonden dat een herverdeling van fibrine van de onderkant naar de bovenkant van trombi gepaard ging met een toegenomen microelasticiteit. Een dergelijke herverdeling werd ook gevonden met bloed van een patiënt met trombocytopenie, waarbij niet zozeer de collageendichtheid, maar het aantal bloedplaatjes de beperkende factor was in het trombusvormend proces. Deze bevindingen tonen dat onder stromingscondities in de aanwezigheid van stolling de hoeveelheid en lokalisatie van de fibrine afhankelijk zijn van de mate van zowel bloedplaatjesadhesie (collageen) als stimulering van de bloedstolling (weefselfactor). De microelasticiteit van een trombus blijkt hierbij bepaald te worden door de fibrinedistributie in de trombus.

In Hoofdstuk 7 hebben we bekeken hoe de intrinsieke stollingscascade die leidt tot factor $\mathrm{X}$ activering bijdraagt aan de trombusvorming met depositie van plaatjes en fibrine trombi onder stromingscondities. Hoewel er sterke aanwijzingen waren dat de fosfatidylserine-exposerende bloedplaatjes in een trombus de activering van protrombine stimuleren, was het veel minder duidelijk hoe de activering van factor X gereguleerd is op een trombus. We vonden dat geactiveerd factor IX en X gelokaliseerd zijn op de fosfatidylserine-exposerende bloedplaatjes in een trombus, terwijl factor VIII co-lokaliseert met VWF, welke op zijn beurt gebonden is aan collageen- en fibrine-vezels. De resultaten wijzen op een mechanisme van aanvoer van VWF-geassocieerd factor VIII naar de fosfatidylserine-exposerende plaatjes, met als resultaat een tijdelijke vorming van het tenase-complex, dat vervolgens de trombusvorming verder bevordert. Perfusie-experimenten met humaan bloed en muizenbloed deficiënt in factor VIII of IX lieten zien dat er een beperkende rol is voor beide factoren in de activering van factor Xa op procoagulante activiteit van bloedplaatjes, en daaropvolgende trombinegeneratie en fibrinevorming. Hoewel hemofilie A en B vaak beschouwd worden als gelijksoortige aandoeningen, laten onze experimenten zien dat de trombusvorming met bloedplaatjes en fibrine sterker 
beperkt werd met factor VIII-deficiënt bloed dan met factor IX-deficiënt bloed. Dit zou kunnen verklaren waarom factor IX deficiëntie overal het algemeen minder gepaard gaat met bloedingscomplicaties.

Aangezien bloedplaatjes de fibrinolysefactor plasminogeen uit hun a-granula kunnen uitscheiden, kan verwacht worden dat zij ook een rol spelen bij de fibrinolyse, dat wil zeggen het oplossen van een fibrine-stolsel. In Hoofdstuk 8 onderzochten we hoe een plaatjes-fibrine trombus gelyseerd kan worden onder stromingscondities, met speciale aandacht daarbij voor de rol van plasminogeen. Microscopische beelden van geïsoleerde bloedplaatjes, opgenomen bij zeer hoge resolutie wezen uit dat plasminogeen voornamelijk gelokaliseerd is op zogenoemde 'caps', die te vinden zijn op fosfatidylserine-exposerende plaatjes. De binding van plasminogeen is afhankelijk van integrine $\alpha_{11 b} \beta_{3}$ en fibrine (of fibrinogeen), aangezien het blokkeren van óf $\alpha_{11 b} \beta_{3}$ óf fibrine-polymerisatie deze interactie tegengaat. In trombi gevormd tijdens stroming van volbloed onder stollingscondities was plasminogeen voornamelijk te vinden op plaatjes-gebonden fibrine, terwijl slechts een klein deel direct bond aan (fosfatidylserine-exposerende) bloedplaatjes. Degradatie van fibrine in de trombi gebeurde dosis-afhankelijk door tissue-type en urokinase-type plasminogeen activator (tPA en UPA), en werd bevorderd door drie processen, namelijk: incorporatie van tPA/uPA in een groeiende trombus, inhibitie van (trombine- en) fibrine-vorming, en verhogen van de stromingssnelheid.

Het afsluitende Hoofdstuk 9 bediscussieert de belangrijkste bevindingen van dit proefschrift in het licht van huidige relevante literatuur. Nieuwe inzichten in de moleculaire mechanismen van trombusvorming, zoals in dit proefschrift, wijzen op onverwacht complexe interacties tussen bloedplaatjes en het stollingssysteem. Verder ophelderen van deze interacties is van belang om bloedingscomplicaties bij verschillende groepen patiënten beter te kunnen begrijpen en behandelen. 



\section{Valorisation}


Knowledge valorisation: "the process of creating value from knowledge, by making knowledge suitable and/or available for social (and/or economic) use and by making knowledge suitable for translation into competitive products, services, processes and new commercial activities" (definition adapted from the National Valorization Committee 2011:8).

The attribution of value is complicated at times as research is split in two distinct fields: basic and applied research. Basic research concerns the process of discovery and aims to expand the knowledge of a particular field of study, with no specific applications toward processes or products in mind. On the other hand, applied research uses a knowledge base - from basic research - to devise solutions for specific practical problems. As a result, it may seem that applied research is more preferable than basic research as it often has a more direct function. However, without the former the latter would have nothing to apply.

My PhD project was dedicated to determine how platelet activation is linked to the dynamic processes of fibrin clot formation and fibrinolysis in haemostasis. Haemostasis is the process that ensures cessation of blood flow after tissue injury. It relies on the activation of both platelets and the coagulation cascade, and results in the formation of a platelet-fibrin thrombus or clot. Bleeding disorders can be caused by disorders of platelet function or number, disorders of clotting factors, and a combination of the two. Most of the work presented here can be considered as basic research with the primary aim to gain more insight into the underlying molecular interaction mechanisms between platelets and coagulation. The valorisation of my thesis will focus on the experimental research described.

In this thesis I employed a sensitive (phospho)proteomics approach as a novel fascinating technique to study platelet function defects. This technique allows the quantification of protein levels and phosphorylation, giving specific information on intracellular pathways upon stimuli. We could identify novel proteins involved in platelet responses, such as aggregation, secretion and thrombus formation, which showed an altered phosphorylation status in a patient with Albright Hereditary Osteodystrophy (Gsa subunit deficiency). Further validation and optimisation of data handling for this technique could render it a valuable new tool to study platelet (signalling) defects. With the new strategies requiring little sample amount they show a great potential to discover, validate and accurately quantify biomarkers in body fluids and primary tissues also in a clinical setting.

Bleeding complications can occur postoperatively due to haemodilution when a patient is heavily transfused in the operating theatre. Although relatively rare, massive blood loss has a high mortality and is therefore clinically important. We found that cardiothoracic patients who suffer from postoperative bleeding complications had elevated levels of von Willebrand factor (VWF), possibly due to the surgical intervention. Interestingly, treatment with desmopressin - a VWF releasing agent - was still beneficial due to release of high-molecular-weight VWF. This increase in large VWF multimers resulted in an improved platelet deposition and thrombus 
formation on collagen under flow, as well as in enhanced platelet procoagulant activity (phosphatidylserine exposure). The results suggest that an increased VWFdependent platelet activity can help to restore haemostasis in these bleeding patients.

Maybe the most well-known link between platelets and the coagulation system is via exposure of phosphatidylserine. We identified an integrin $\alpha_{11 b} \beta_{3}$ outside-in signalling pathway that stimulates the late phase of the $\mathrm{Ca}^{2+}$ responses in platelets, thereby increasing phosphatidylserine exposure and enforcing tissue factor-triggered thrombin generation on platelets. In agreement with inhibitor studies, we found that platelets from patients with Glanzmann's thrombasthenia, lacking $\alpha_{11 b} \beta_{3}$, possessed a reduced procoagulant potential. We thus showed how integrin $\alpha_{11 b} \beta_{3}$ plays an important role in the development of platelet procoagulant activity, in addition to its classical role in platelet aggregate formation. This indicates that inhibitors of $\alpha_{111} \beta_{3}$ will not only limit platelet function but will also diminish the coagulant potential.

In the final chapters of my thesis I developed and employed an integrative whole blood perfusion method, allowing the combined assessment of platelet and coagulant functions, aswellastheprocessoffibrinolysis. Thisallowedustoassesstheformationand composition of fibrin clots and lysis on various thrombogenic surfaces. As currentassays mostly focus on either platelet function or coagulant capacity under static conditions, this new assay may assist in the indication of bleeding (or thrombogenic) tendencies.

While it is known that platelet- and fibrin-dependent thrombus formation is regulated by blood flow, blood components, and exposure of collagen and tissue factor, the interactions between these blood-borne and vascular components are not well understood. We demonstrate for the first time that under flow conditions platelet and fibrin amount and localisation depend on the abundance of triggers for platelet adhesion (collagen) and coagulation (tissue factor). A novel nanoindentation technique, to assess the mechanical properties - i.e. the microelasticity - of thrombi furthermore showcased that thrombus elasticity relies on the plateletfibrin distribution. Importantly, we showed a reduced fibrin-thrombus formation for patients with diverse haemostatic defects. In addition, I studied the lysis of platelet-fibrin thrombi under flow conditions, with particular focus on the role of plasminogen herein. In thrombi formed by whole blood perfusion plasminogen primarily bound to platelet-associated fibrin, with a smaller pool directly binding to platelets. Degradation of fibrin in the thrombi was dose-dependently induced by tissue and urokinase plasminogen activator (tPA/UPA), and was promoted by three distinct factors, namely by: incorporation of tPA/uPA into the growing thrombus, inhibition of (thrombin and) fibrin formation, and increasing the flow rate.

One haemostatic defect studied in more detail is Haemophilia. Whereas haemophilia A and B (deficiency in coagulation factor VIII and IX, respectively) are often consideredassimilarpathologies, our dataindicated that the formation of platelet-fibrin thrombi was less reduced with factor IX-deficient blood than with factor VIII-deficient blood. This might explain the lesser bleeding complications in patients with factor IX deficiency. Perfusion experiments indicated a limiting role for both factors in the factor 
Xa-mediated stimulation of platelet procoagulant activity via thrombin generation and subsequent fibrin formation, even in the presence of high extrinsic stimulation. As clot stability depends on both its elastic and fibrinolytic properties, a better understanding of the mechanisms underlying these characteristics is essential for our understanding of haemostasis. 

Curriculum vitae 


\section{About the author}

Frauke Swieringa was born on October $23^{\text {rd }} 1984$ in Heerlen, The Netherlands. In 1996 she started her secondary school education at the Sintermeerten College in Heerlen, where she obtained her VWO-diploma in 2003 for Nature \& Health with philosophy in her free section. In 2004 she started her bachelor study Molecular Lifesciences at the University of Maastricht. After receiving her bachelor degree in 2008, she started a master Clinical Molecular Sciences at the same university. She followed her graduate internship, focussing on the identification of blood platelet-reactive sites in collagen, at the Department of Biochemistry at the University of Maastricht and the Biochemistry Department of the University of Cambridge (United Kingdom). This resulted in a master-diploma in 2010 with highest degree, cum laude. In November 2010 she started her PhD research in the Cardiovascular Research Institute Maastricht (CARIM) at the Department of Biochemistry, supervised by Prof. Dr. J.W.M. Heemskerk and Dr. P.E.J. van der Meijden. Here she conducted research in the field of platelets, thrombosis and haemostasis, as described in this thesis. As a PhD-student, she presented her research at international conferences in Kyoto (Japan), Maastricht (The Netherlands), Bath (United Kingdom) and Amsterdam (The Netherlands) and received a young investigator award. Currently, she is working at the Department of Biochemistry at the University of Maastricht as post-doctoral researcher. 


\section{Over de auteur}

Frauke Swieringa werd geboren op 23 oktober 1984 te Heerlen. In 1996 begon zij haar middelbare schoolopleiding aan het Sintermeerten College te Heerlen, waar zij in 2003 haar VWO-diploma behaalde met als afstudeerprofiel Natuur \& Gezondheid met Filosofie in het vrije deel. In 2004 begon zij de bacheloropleiding Moleculaire Levenswetenschappen aan de Universiteit Maastricht. Na het behalen van haar bachelor-graad in 2008, volgde zij aan dezelfde universiteit de masteropleiding Clinical Molecular Sciences. Haar afstudeerstage naar het identificeren van bloedplaatjesactieve domeinen in collageen voerde zij uit bij de vakgroep Biochemie van de Universiteit Maastricht en de vakgroep Biochemie van de Universiteit van Cambridge (Groot-Brittannië). Hierna ontving zij in 2010 haar master-diploma met predicaat cum laude. In november 2010 begon zij haar promotieonderzoek binnen het Cardiovascular Research Institute Maastricht (CARIM) bij de vakgroep Biochemie onder leiding van Prof. Dr. J.W.M. Heemskerk en Dr. P.E.J. van der Meijden. Hier voerde zij onderzoek uit op het gebied van bloedplaatjes, trombose en hemostase, zoals beschreven in dit proefschrift. Als promovendus presenteerde zij haar onderzoek op internationale congressen in Kyoto (Japan), Maastricht, Bath (Groot-Brittannië) en Amsterdam en kreeg daarvoor een young investigator award. Momenteel is zij werkzaam bij de vakgroep Biochemie aan de Universiteit Maastricht als post-doctoraal onderzoeker. 



\section{Publications}




\section{Full papers}

1. Van der Meijden PEJ, Feijge MAH*, Swieringa F*, Gilio K, Nergiz-Unal R, Hamulyák $\mathrm{K}$, Heemskerk JWM (* equal contribution). Key role of integrin $\alpha_{11 b} \beta_{3}$ signaling to Syk kinase in tissue factor-induced thrombin generation. Cell Mol Life Sci. 2012;69:3481-3492.

2. Van Kruchten R, Mattheij NJA, Saunders C, Feijge MAH, Swieringa F, Wolfs JLN, Collins PW, Heemskerk JWM, Bevers EM. Both TMEM16F-dependent and TMEM16F-independent pathways contribute to phosphatidylserine exposure in platelet apoptosis and platelet activation. Blood. 2013;121:1850-1857.

3. Swieringa F, Kuijpers MJE, Heemskerk JWM, Van der Meijden PEJ. Targeting platelet receptor function in thrombus formation: The risk of bleeding. Blood Rev. 2014;28:9-21.

4. De Witt SM, Swieringa F, Cavill R, Lamers MME, Van Kruchten R, Mastenbroek T, Baaten C, Coort S, Pugh N, Schulz A, Scharrer I, Jurk K, Zieger B, Clemetson KJ, Farndale RW, Heemskerk JWM, Cosemans JMEM. Identification of platelet function defects by multi-parameter assessment of thrombus formation. Nat Commun. 2014, 5: 4257.

5. De Witt S, Swieringa F, Heemskerk J, Cosemans J. Multi-parameter assessment of thrombus formation on microspotted arrays of thrombogenic surfaces. Nat Prot Exchange. 2014; DOI: ISSN 2043-0116.

6. Whyte CS, Swieringa F, Mastenbroek TG, Lionikiene AS, Lancé MD, Van der Meijden PEJ, Heemskerk JWM, Mutch NJ. Plasminogen associates with phosphatidylserine-exposing platelets and contributes to thrombus lysis under flow. Blood. 2015;125:2568-2578.

7. Swieringa F, Kuijpers MJE, Lamers MM, Van der Meijden PEJ, Heemskerk JWM. Rate-limiting roles of the tenase complex of factors VIII and IX in platelet procoagulant activity and formation of platelet-fibrin thrombi under flow. Haematologica. 2015;100:748-756.

8. Swieringa F, Lancé MD, Fuchs B, Feijge MAH, Solecka BA, Verheijen LPJ, Hughes KR, Van Oerle R, Deckmyn H, Kannicht C, Heemskerk JWM, Van der Meijden PEJ. Desmopressin treatment improves platelet function under flow in patients with postoperative bleeding. J Thromb Haemost. 2015;13:1503-1513.

9. Baaten CCFMJ, Veenstra LF, Wetzels R, Van Geffen JP, Swieringa F, De Witt SM, Henskens YMC, Crijns H, Nylander S, Van Giezen JJJ, Heemskerk JWM, Van der Meijden PEJ. Gradual increase in thrombogenicity of juvenile platelets formed upon offset of prasugrel medication. Haematologica, 2015;100:1131-1138.

10. Mastenbroek TG, Feijge MAH, Kremers RWM, Van den Bosch MT, Swieringa F, De Groef L, Moons L, Bennett C, Gheveart C, Johnson JL, Van der Meijden PEJ, Cosemans JMEM. Platelet-associated matrix metalloproteinases regulate thrombus formation and exert local collagenolytic activity. Arterioscler Thromb Vasc Biol, 2015;35:2554-2561. 
11. Mattheij NJA, Swieringa F, Mastenbroek TG, Berny-Lang MA, May F, Baaten, CCFMJ, Van der Meijden PEJ, Henskens YMC, Beckers EAM, Suylen DPL, Nolte MW, Hackeng TM, McCarty OJT, Heemskerk JWM, Cosemans JMEM. Coated platelets function in platelet-dependent fibrin formation via integrin $\alpha_{11 b} \beta_{3}$ and transglutaminase factor XIII. Haematologica, 2015; pii: haematol.2015.131441.

12. Swieringa F, Baaten CCFMJ*, Verdoold $R^{*}$, Mastenbroek TG, Rijnveld R, Van der Laan KO, Breel EJ, Collins PW, Lancé MD, Henskens YMC, Cosemans JMEM, Heemskerk JWM, Van der Meijden PEJ (* equal contribution). Platelet control of fibrin distribution and micro-elasticity in thrombus formation under flow. Arterioscler Thromb Vasc Biol, 2016; pii: ATVBAHA.115.306537.

13. Van Geffen JP, Swieringa F, Heemskerk JWM. Platelets and coagulation in thrombus formation: aberrations in the Scott syndrome. Thromb Res, 2016;accepted.

14. Solari FA, Mattheij NJA, Burkhart J, Swieringa F, Collins PW, Cosemans JMEM, Sickmann A, Heemskerk JWM, Zahedi RP. Altered protein expression, phosphorylation and cleavage in platelets from rare Scott syndrome patient revealed by quantitative proteomics analysis. Submitted.

15. Swieringa F, Solari FA, Feijge MAH, Mattheij NJA, Stumpel CTRM, Sickmann A, Van der Meijden PEJ, Körver-Keularts IMLW, Zahedi RP, Heemskerk JWM. Multiple changes in novel protein kinase A phosphorylation targets identified in platelets from patient with impaired Gsa signalling. Submitted.

\section{Abstracts}

1. Swieringa F, Feijge MA, Henskens YM, Schrander-Stumpel CT, Rubio-Gozalbo ME, Van der Meijden PE, Keularts IM, Heemskerk JW. Common signaling pathways involving $\mathrm{PI} 3 \mathrm{~K}$ beta in the regulation of platelet inhibition via Gs in subjects with normal or altered Gs function. XXIII ${ }^{\text {th }}$ Congress of the International Society for Thrombosis and Haemostasis (ISTH), Kyoto, Japan, 2011. J Thromb Haemost, Suppl. July 2011.

2. De Witt SM, Lamers MM, Nergiz R, Swieringa F, Westein E, Cosemans JM, Heemskerk JW. Platelet receptors in thrombus formation on spotted microarray surfaces. XXIII ${ }^{\text {th }}$ Congress of the ISTH, Kyoto, Japan, 2011. J Thromb Haemost, Suppl. July 2011.

3. De Witt SM, Lamers MM, Swieringa F, Westein E, Farndale RW, Clemetson KJ, Cosemans JM, Heemskerk JW. Platelet receptors in thrombus formation on spotted microarray surfaces: a role for CLEC-2. Proc. $13^{\text {th }}$ United Kingdom Platelet Meeting, Cardiff, United Kingdom, 2011.

4. De Witt SM, Lamers MM, Swieringa F, Van Kruchten R, Farndale RW, Clemetson KJ, Cosemans JM, Heemskerk JW. Synergy of platelet receptors in thrombus formation: a microarray approach. $58^{\text {th }}$ Meeting of SSC/ISTH, Liverpool, United Kingdom, 2012. 
5. Swieringa F, Kuiper GJ, Lancé MD, Heemskerk JW, Van der Meijden PE. Desmopressin treatment improves thrombus formation in patients undergoing cardiothoracic surgery. Proc. $1^{\text {st }}$ European Platelet Network Conference, EUPLAN, Maastricht, The Netherlands, 2012.

6. De Witt SM, Lamers MM, Swieringa F, Van Kruchten R, Farndale RW, Clemetson $\mathrm{KJ}$, Cosemans JM, Heemskerk JW. Multiparameter assessment of thrombus formation: A systems biology approach. Proc. $1^{\text {st }}$ European Platelet Network Conference, EUPLAN, Maastricht, The Netherlands, 2012.

7. Swieringa F, Kuiper GJ, Lancé MD, Heemskerk JW, Van der Meijden PE. Thrombus formation under flow conditions in enhanced after desmopressin treatment in patients with postoperative bleeding complications. Proc. British Society for Haemostasis \& Thrombosis and the UK Platelet Annual Joint Scientific Meeting, Bath, United Kingdom, 2012.

8. Swieringa F, Kuijpers MJ, Lamers MM, Renné T, Heemskerk JW, Van der Meijden PE. Contribution of intrinsic and extrinsic coagulation pathways to whole blood clot and thrombus formation under flow. XXIV th Congress of the ISTH, 2013, Amsterdam, The Netherlands. J Thromb Haemost, Suppl. June 2013.

9. Kuijpers MJ, Mattheij NJ, Cipolla L, Cosemans JM, Lievens D, Donners M, Swieringa F, Mastenbroek TG, Torti M, Lutgens E, Heemskerk JW. A role for CD4OL signalling in platelet activation and thrombus formation that is independent of CD40. XXIV th Congress of the ISTH, 2013, Amsterdam, The Netherlands. J Thromb Haemost, Suppl. June 2013.

10. Farndale RW, Swieringa F, Tsim M, Howes J, Jung S, Bihan D, Hamaia S. XXIVth Congress of the ISTH , 2013, Amsterdam, The Netherlands. J Thromb Haemost, Suppl. June 2013.

11. De Witt SM, Lamers MM, Swieringa F, Van Kruchten R, Mastenbroek TG, Coort S, Cavill R, Pugh N, Farndale RW, Clemetson KJ, Heemskerk JW, Cosemans JM. Advanced multi-parameter assessment of microspot thrombus formation: A systems biology approach. XXIVth Congress of the ISTH, 2013, Amsterdam, The Netherlands. J Thromb Haemost, Suppl. June 2013.

12. Vignoli A, Van der Meijden PE, Giaccherini C, Swieringa F, Marchetti M, Ten Cate $\mathrm{H}$, Heemskerk JW, Falanga A. Platelet adhesion to collagen under flow conditions is increased in essential thrombocythemia patients. XXIVth Congress of the ISTH, 2013, Amsterdam, The Netherlands. J Thromb Haemost, Suppl. June 2013.

13. Whyte CS, Swieringa F, Heemskerk JW, Mutch NJ. Plasminogen preferentially binds to platelet-associated fibrin. British Society for Haemostasis and Thrombosis / Belgian Society on Thrombosis and Haemostasis Joint Meeting, Nottingham, United Kingdom, 2013.

14. Whyte CS, Swieringa F, Heemskerk JW, Mutch NJ. A flow model study of the role of plasminogen in thrombus lysis. Scottish Cardiovascular Forum, Aberdeen, Scotland, 2014.

15. Whyte CS, Swieringa F, Van der Meijden PE, Heemskerk JW, Mutch NJ. The 
distribution and function of plasminogen in thrombus lysis under flow. $22^{\text {nd }}$ International Congress on Fibrinolysis and Proteolysis, Marseille, France, 2014.

16. Mattheij NJ, Solari FA, Burkhart J, Swieringa F, Collins PW, Cosemans JM, Sickmann A, Zahedi RP, Heemskerk JW. Altered phosphorylation profile of Scott syndrome platelets as revealed by quantitative phosphoproteomics. Proc. $2^{\text {nd }}$ European Platelet Network Conference, EUPLAN, Le Bischenberg, France, 2014.

17. Mattheij NJ, Solari FA, Burkhart J, Swieringa F, Collins PW, Cosemans JM, Sickmann A, Zahedi RP, Heemskerk JW. Altered phosphorylation profile of Scott syndrome platelets as revealed by quantitative phosphoproteomics. Proc. HUPO $13^{\text {th }}$ Annual World Congress, Madrid, Spain, 2014.

18. Solari FA, Mattheij NJ, Burkhart F, Swieringa F, Collins CW, Cosemans JM, Sickmann A, Heemskerk JW, Zahedi RP. Calpain-dependent cleavage in highly activated platelets revealed by quantitative $\mathrm{N}$-terminal ChaFRADIC|: alterations in Scott syndrome. Proc. HUPO $13^{\text {th }}$ Annual World Congress, Madrid, Spain, 2014.

19. Whyte CS, Swieringa F, Mastenbroek TG, Lionikiene AS, Lancé MD, Van der Meijden PE, Heemskerk JW, Mutch NJ. Plasminogen localizes to distinct caps on phosphatidylserine-exposing platelets and promotes thrombus lysis under flow. $X X V^{\text {th }}$ Congress of the ISTH, Toronto, Canada, 2015.

20. Swieringa $F$, Lancé MD, Fuchs B, Feijge MA, Solecka BA, Verheijen LP, Hughes KR, Deckmyn H, Kannicht C, Heemskerk JW, Van der Meijden PE. Desmopressin treatment improves platelet function under flow in patients with postoperative bleeding. XXV th Congress of the ISTH, Toronto, Canada, 2015.

21. Mattheij NJ, Solari FA, Burkhart J, Swieringa F, Collins PW, Cosemans JM, Sickmann A, Zahedi RP, Heemskerk JW. Scott syndrome platelets show alterations in protein expression levels, calcium-dependent phosphorylation and cleavage as revealed by proteomics. XXV th Congress of the ISTH, Toronto, Canada, 2015.

22. Swieringa F, Kuijpers MJ, Lamers MM, Van der Meijden PE, Heemskerk JW. Ratelimiting roles of tenase complex of factors VIII and IX in platelet procoagulant activity and formation of platelet-fibrin thrombi under flow. XXVth Congress of the ISTH, Toronto, Canada, 2015.

23. Mattheij NJ, Swieringa F, Berny-Lang MA, May F, Van der Meijden PE, Suylen DP, Nolte MW, Hackeng TM, McCarty OJ, Heemskerk JW, Cosemans JM. Combined roles of factor XIIla and glycoprotein IIB/IIIA in platelet-dependent fibrin formation. $X X V^{\text {th }}$ Congress of the International Society of Thrombosis and Haemostasis, Toronto, Canada, 2015.

24. Baaten CCFMJ, Henskens YMC, Swieringa F, Wtzels R, Van Oerle R, Ten Cate H, Moenen FCJI, Beckers EAM, Heemskerk JWM, Van der Meijden PEJ. Multiple functional defects in platelets from thrombocytopenic cancer patients undergoing chemotherapy. $8^{\text {th }}$ International conference on thrombosis and hemostasis issues in cancer, Bergamo, Italy, 2015.

25. Solari FA, Venne S, Mattheij NJ, Burkhart J, Swieringa F, Collins PW, Cosemans 
JMEM, Sickmann A, Heemskerk JWM, Zahedi RP. N-Terminal Charged-based fractional diagonal chromatography (ChaFRADIC) to study proteolytic events in procoagulant platelets. $64^{\text {th }}$ Conference of the American Society for Mass Spectrometry, San Antonio TX, USA .

\section{Oral presentations}

1. Swieringa F. Common signaling pathways involving $\mathrm{PI} 3 \mathrm{~K}$ beta in the regulation of platelet inhibition via Gs in subjects with normal or altered Gs function. XXIII ${ }^{\text {th }}$ Congress of the ISTH, Kyoto, Japan, 2011.

2. Swieringa F. Contribution of intrinsic and extrinsic coagulation pathways to whole blood clot and thrombus formation under flow. XXIV th Congress of the ISTH, Amsterdam, The Netherlands, 2013.

\section{Awards}

Young Investigators Award. XXIII ${ }^{\text {th }}$ ISTH, Kyoto, Japan, 2011. 

Dankwoord 
Je realiseert je soms ineens dat je een bofkont bent, iets dat ik besef tijdens het schrijven van mijn dankwoord. Ik heb een lijstje gemaakt met mensen die ik graag wil bedanken, mooi onderverdeeld in collega's van het superplatelet team, Biochemie en andere afdelingen als ook vrienden en familie. Een verdeling die prettig gecompliceerd is, omdat veel collega's ook vrienden zijn geworden. De lijst van mensen die mij hebben geholpen, naar mijn gejammer hebben geluisterd, geïnformeerd hebben naar de voortgang van mijn boekje, of mij even lieten vergeten dat ik aan het promoveren was, lijkt eindeloos. Toen wist ik: ik ben een bofkont!

Allereerst wil ik heel graag mijn promotieteam bedanken. Mijn promotor, Prof. Johan Heemskerk. Beste Johan, als promotor had ik waarschijnlijk geen betere kunnen treffen. Je liet weten dat ik altijd hysterisch binnen mocht rennen en dat gaf mij rust. Ik mocht altijd even storen, zowel met een serieuze vraag als ook met het ventileren van paniek. Ik heb onze samenwerking altijd als zeer prettig ervaren, bedankt voor alles, van bachelor tot PhD. Mijn co-promotor, Paola van der Meijden. Lief Paoolsjen, lieve collega, maar vooral lieve vriendin, ik kan me niet voorstellen hoe de afgelopen $5^{+}$jaren zouden zijn geweest zonder jou. Het was zeker een stuk minder gezellig geweest. Er is vooral heel veel gelachen en soms gehuild (ik dan). Toen ik de grens van 30 bereikte en bij de aankoop van mijn eerste huis was jij de persoon die me met heel veel roze bij stond. Ik weet zeker dat als we later grijs zijn, we nog steeds vrolijk door kwebbelen. Dankjewel dat je me af en toe een schop onder m'n kont gaf als ik dat nodig had. Vooral bedankt dat je dan vervolgens zei: "Sorry dat ik even bazig deed." Je bent een lieverd, dankjewel voor alles!

Leden van de leescommissie, Prof. Harry Schouten, Prof. Robert Ariëns, Prof. Richard Farndale, Dr. Yvonne Henskens en Dr. Rory Koenen. Thank you all very much for reviewing my thesis. Ik waardeer de gesprekken met betrekking tot mijn proefschrift en natuurlijk de gezellige gesprekken daar buiten. Dear Richard, thank you for giving me the opportunity to come to your lab in Cambridge. Not only was it a great learning experience, it was also a lot of fun thanks to you and the great people in your group.

Lieve paranimfen, Kelly en Connie! Kel, mede-prinses, alles wat ik hier zou typen zou tekort doen aan onze vriendschap. Het is bizar hoeveel we al samen hebben meegemaakt. Dansen in onesies, Disney films kijken, taarten bakken, Baileys drinken en vooral heel veel kletsen. Ik kan alleen maar zeggen dat ik hoop dat dit altijd zo blijft! Constance, sorry voor de bijnaam... Met jou samenwerken was altijd geslaagd en vooral erg gezellig. Je bent een ontzettende lieverd, altijd bereidt om iedereen te helpen, altijd vriendelijk en vergeleken met mij zo heerlijk rustig. Ik bof dat jullie mijn nimfjes zijn!

Marion, Judith en Marijke, de drie wijzen, bedankt dat jullie altijd bereid waren om jullie kennis te delen. Marion, de stap die je hebt gemaakt naar een andere baan vind ik zeer stoer, maar ik mis onze fijne gesprekken. Judith, dankjewel voor je eerlijkheid. 
Jij wist me meerdere keren te wijzen op punten die ik zelf niet zag. Ik wilde het misschien niet altijd horen, maar het heeft me zeker geholpen. Ik kon altijd even bij je binnen stappen voor een goed en gezellig babbeltje. Marijke, ik waardeer al jouw hulp van de afgelopen jaren. Dankjewel voor de prinsessentaart, beste taart ooit!

Al mijn lieve (ex-)kamergenootjes: Moniek, Roger, Tom, Ankie en Remco. Allereerst de mannen: dank dat jullie me nooit de deur uit hebben gezet! Jullie hebben heel wat muziek moeten aanhoren die de gemiddelde man gek zou maken. Roger, je vertrek was een groot gemis, want er bleef niemand over die met mij over sport wilde praten. Dankzij jouw goedbedoelde aandringen heb ik wel eindelijk een fiets gekocht, na het voorwerp nu nog de handeling. Tom, bedankt voor het opstellen en vervolgens totaal negeren van de 12-uur regel. Ik heb altijd met veel plezier met je samengewerkt (en zal nooit meer met een nietmachine gooien in jouw bijzijn). Remco, dank voor je geduld om me (technische) dingen uit te leggen, maar nog meer voor alle nietnuttige informatie waarmee jij mij hebt verrijkt. Ik heb veel van je geleerd! Moniek, met jou op een kamer was het altijd feest: kerstmuziek zodra de Sint het land uit was, carnavalsmuziek vanaf de elfde-van-de-elfde of Disney krakers. Hopelijk kunnen we nog lang samen de economie stimuleren met onze shopmiddagjes. Lieve Ankie, mede-stollingsbikkel met uitstekende muzieksmaak, dankjewel voor de gezellige babbeltjes. Ik hoop dat ik nog een tijd mee kan kijken met al je leuke proeven.

Alle lieve mensen van de 'andere AIO-kamer': Nadine, Susanne, Magdi, Jilke en Erik. Nadine, proteomics partner in crime, jij maakte dat stomme FACSen toch altijd erg gezellig. Samen hebben we heel wat mooie proeven gedaan... Samen hebben we ook heel professioneel proeven verknald, maar het was in ieder geval altijd erg gezellig! Susanne, lieve Suus, wat was ik sip toen jij vertelde dat je weg ging. Ik denk dat het tripje naar Cambridge dat benadrukte: samen lekker dansen, keihard zingen en heerlijk veel eten. Dear Magdi, hoi Magdi! Meisje dat mij Palenka in Nederland gaf. I am really happy you joined us again, it has been more fun with you here! En natuurlijk ook dankjewel voor alle hulp en gezelligheid aan Jilke en Erik. Erik, het is mij uiteindelijk toch gelukt om de ZEISS te hanteren zonder jouw nummer op speed dial. Bij Biochemie 'kamerloze' Siamack, lieve Mackintosh, wij hebben elkaar altijd weten te vinden. Eerst samen op een basisschool in Nuth, daarna studiegenoten bij de bachelor Moleculaire Levenswetenschappen. We hebben zelfs nog even samengewoond (gezang en sjnaps alom) en dan ook deels samen promoveren. Ik ben blij dat je er steeds was!

Een groot dankjewel aan alle studenten die mee hebben geholpen aan de hoofdstukken in mijn proefschrift. Maaike, Yannick, Lieke en Kevin, wat fijn dat jullie er waren, niet alleen voor de hulp, maar ook voor de gezelligheid.

Alle lieve mensen van het superplatelet team: Dankjewel dat ik in zo'n heerlijke groep mocht werken! 
Alle mede-AIOs van Biochemie, dankjewel voor de vele, vele leuke babbeltjes. Het lijkt alsof ik alleen maar gebabbeld heb 5 jaar lang... gelukkig kan ik multitasken. Lieve (de liefste, ik mis je in Maasje) Rinske, Marie-Claire, Geert-Jan, Barbara, Martijn, Brecht, Annemieke, Pieter, Francesca, Minka, Suzanne, Farida, Tanja, Annemiek, Daniëlle, Rick, Armand, Stijn, Jelle, Peter, Nahla, Stepan en natuurlijk alle anderen, bedankt!

En natuulijk alle anderen van Biochemie, een aantal hele fijne mensen op een rij: Stella, Tilman, Betta, Roy, Gerrie, Dennis, Ingrid, Lidewij, Liset, Leon, Chris, mijn buurmannen Niko, René en Henry, Diane, Stephanie, Marieke, Patricia, Hugo, Jan, Guido, Edouard, José, Simone, Cecile, Petra, Gosia, Hans, Alexandra, Kanin en vooral lieve tante Trees. Dankjewel, iedereen van Biochemie, wat een topgroep zijn we toch!

Heel veel dank aan alle andere mensen die nog niet genoemd zijn waar ik mee heb mogen publiceren. Marcus en Connie bedankt voor jullie belangrijke klinische visie op mijn onderzoek. Ook wil ik graag Dr. Karly Hamulyák bedanken. Oud AIOs, Reyhan en Karen, jullie bedankt voor het doorgeven van de kennis. Dear Dr. Birte Fuchs, Barbara Solecka, Dr. Christoph Kannicht and Prof. Hans Deckmyn, thank you for all your help on the desmopressin chapter! De mannen van Optics11, Koen, Niek en Ernst, dank dat jullie mijn thesis versterkt hebben met de kracht van nanoindentatie. Prof. Peter Collins, thank you for your contribution. Lieve Irene, al vanaf mijn stage tijdens de master Clinical Molecular Science mag ik met jou samenwerken en dit heb ik altijd met heel veel plezier gedaan. Dear Prof. Nikki Mutch, Ausra Lionikiene, and Dr. Claire Whyte, thank you so very much for widening my horizon to the lovely field of fibrinolysis! Prof. Albert Sickmann, Dr. René Zahedi and dear Fiorella Solari, although sometimes meetings seemed to be in Chinese, I did learn a lot from you all. It is a great pleasure working with you!

Een speciaal woord van dank aan alle mensen die in de afgelopen jaren bloed hebben gedoneerd of bloed hebben geprikt voor mijn onderzoek. Zonder jullie was dit hele proefschrift er nooit geweest.

Buiten de werkplek zijn er ook hele lieve mensen die ik graag wil bedanken. Marti, lieve Matseflats, wat ben ik blij dat ik je na het Sintermeerten college weer zag op de universiteit in Maastricht! Samenwonen met Jekerbabes Daan en Loes, samen op vakantie, samen een snurkje doen tijdens college, samen carnaval vieren en zo kan ik nog 1000 pagina's vullen met redenen waarom ik dol op je ben! Dennis, ik ben de afgelopen 10 jaar zo gewend aan het feit dat je er altijd was. Als je dan besluit naar Amerika te gaan, zoek ik je daar natuurlijk op. Bedankt dat ik je mag missen, dat lijkt me een goed teken voor onze vriendschap, maar kom wel weer terug. Lieve Femke, Thijs, Kimberley, Roel, Robin en Nadine, bedankt voor alle gezellige momenten die ontelbaar waren en waar hopelijk nog iemand ooit een boek aan gaat wijden. Ik heb met jullie mogen genieten van wijn, muziek, diners, feestjes en andere ongein. 
Lief Oud-Brunssum, Jana \& René, Anke \& Rob, Loek \& Bianca, Jesper \& Marijke, Wendy \& Herold, Pieter \& Wendy, Ralph \& Simone, special guests Niels \& Tess, Kay \& Rachèl en Steffie \& Roel, bedankt voor alle keren dat jullie informeerden hoe het nu ging met mijn promoveren! Maar vooral bedankt voor alle mooie feestjes, weekendjes weg en vakanties in de tussentijd!

Lief Jong-Brunssum, Lindsay \& Rocco, Jackie \& Bram, Melanie \& Thomas, super Jill en natuurlijk ook Kel \& Bart. Liefste Tjiksies, met jullie is het altijd feest, zeker met de beste $C D$ ooit! Ik koester onze meiden-momentjes, die houden me jong, haha. Stay fabulous and....

Mijn familie, Père de la peer, Mère de la mer, Kikkie, Puk en natuurlijk Elisan, Kim en m'n lieve neefjes Stan en Ties en nichtje Veere en mijn schone-tweede-familie waar ik zo ontzettend mee geboft heb. Super cliché, maar echt niet minder waar: dank voor alle steun! Vooral ook bedankt voor alle gekkigheid, de interesse in mijn onderzoek, de gezellige borreltjes, het lego bouwen en de 1000-liefde! En mama, dankjewel voor de prachtige cover!

En dan Patrick, de puupie. Liefste lieverd, dankjewel voor werkelijk alles. Dankjewel voor het samen volslagen maf doen, voor het geduld dat je met me hebt, voor het samen genieten, voor het feit dat het je een mooi plan lijkt om je leven met mij te delen! Had ik al gezegd dat ik een bofkont ben?

Ik ben een bofkont, dankjewel allemaal! 
